\title{
Manuel de l'OCDE sur les statistiques des brevets
}
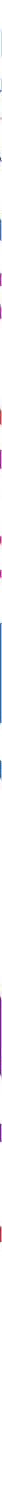



\section{Manuel de l'OCDE sur les statistiques des brevets}




\section{ORGANISATION DE COOPÉRATION ET DE DÉVELOPPEMENT ÉCONOMIQUES}

L'OCDE est un forum unique en son genre où les gouvernements de 30 démocraties œuvrent ensemble pour relever les défis économiques, sociaux et environnementaux que pose la mondialisation. L'OCDE est aussi à l'avant-garde des efforts entrepris pour comprendre les évolutions du monde actuel et les préoccupations qu'elles font naître. Elle aide les gouvernements à faire face à des situations nouvelles en examinant des thèmes tels que le gouvernement d'entreprise, l'économie de l'information et les défis posés par le vieillissement de la population. L'Organisation offre aux gouvernements un cadre leur permettant de comparer leurs expériences en matière de politiques, de chercher des réponses à des problèmes communs, d'identifier les bonnes pratiques et de travailler à la coordination des politiques nationales et internationales.

Les pays membres de l'OCDE sont : l'Allemagne, l'Australie, l'Autriche, la Belgique, le Canada, la Corée, le Danemark, l'Espagne, les États-Unis, la Finlande, la France, la Grèce, la Hongrie, l'Irlande, l'Islande, l'Italie, le Japon, le Luxembourg, le Mexique, la Norvège, la Nouvelle-Zélande, les Pays-Bas, la Pologne, le Portugal, la République slovaque, la République tchèque, le Royaume-Uni, la Suède, la Suisse et la Turquie. La Commission des Communautés européennes participe aux travaux de l'OCDE.

Les Éditions OCDE assurent une large diffusion aux travaux de l'Organisation. Ces derniers comprennent les résultats de l'activité de collecte de statistiques, les travaux de recherche menés sur des questions économiques, sociales et environnementales, ainsi que les conventions, les principes directeurs et les modèles développés par les pays membres.

Cet ouvrage est publié sous la responsabilité du Secrétaire général de l'OCDE. Les opinions et les interprétations exprimées ne reflètent pas nécessairement les vues de l'OCDE ou des gouvernements de ses pays membres.

Publié en anglais sous le titre :

\section{OECD Patent Statistics Manual}

Les corrigenda des publications de l'OCDE sont disponibles sur : www.oecd.org/editions/corrigenda.

(C) OCDE 2009

Illustration de couverture :

(c) Rian Hughes/Stockbyte/Getty Images

Vous êtes autorisés à copier, télécharger ou imprimer du contenu OCDE pour votre utilisation personnelle. Vous pouvez inclure des extraits des publications, des bases de données et produits multimédia de l'OCDE dans vos documents, présentations, blogs, sites Internet et matériel d'enseignement, sous réserve de faire mention de la source OCDE et du copyright. Les demandes pour usage public ou commercial ou de traduction devront être adressées à rights@oecd.org. Les demandes d'autorisation de photocopier une partie de ce contenu à des fins publiques ou commerciales peuvent être obtenues auprès du Copyright Clearance Center (CCC) info@copyright.com ou du Centre français d'exploitation du droit de copie (CFC) contact@cfcopies.com. 


\section{Avant-propos}

C

manuel a été préparé par le Secrétariat de l'OCDE conjointement avec le Groupe de travail des experts nationaux sur les indicateurs de science et de technologie (GENIST) afin de donner aux utilisateurs et producteurs de statistiques sur les brevets des indications de base pour la compilation et l'analyse de ces données.

L'intérêt que l'OCDE attache à l'exploitation des statistiques sur les brevets remonte à la fin des années 1970. L'OCDE a publié une première édition de ce manuel en 1994. A la conférence "Blue Sky » sur « les nouveaux indicateurs de la science et de la technologie pour une économie fondée sur le savoir », organisée par l'OCDE en 1996, les experts ont estimé que les brevets offraient une voie prometteuse pour améliorer notre compréhension quantitative des activités scientifiques et technologiques dans un contexte économique et politique qui évolue rapidement. Depuis lors, les travaux statistiques sur les brevets ont sensiblement progressé, à l'OCDE, dans les pays membres et dans le monde universitaire. Le présent manuel prend en compte ces évolutions.

Le manuel montre les utilisations auxquelles les statistiques des brevets se prêtent et ne se prêtent pas, et comment dénombrer les brevets pour maximiser l'information sur les activités scientifiques et techniques tout en limitant le plus possible le «bruit » et les « biais ». Les données des brevets apportent des éclairages uniques sur les résultats des activités d'invention (p. ex. la localisation des activités inventives, les réseaux d'invention, les technologies émergentes, etc.). Conjugués à d'autres données, elles épaulent l'analyse d'autres dimensions de l'innovation qui intéressent les responsables de l'action publique, comme le rôle de la propriété intellectuelle dans les performances économiques, l'entrepreneuriat, et le suivi des interactions dans le système scientifique et technologique. Cependant, les indicateurs fondés sur les brevets présentent plusieurs points faibles et ils doivent donc être conçus et interprétés avec soin, d'où le besoin de ce manuel.

Le Manuel de l'OCDE sur les statistiques des brevets appartient à la série des manuels de Frascati de l'OCDE consacrés à la mesure des activités scientifiques et technologiques, parmi lesquels figurent notamment le Manuel de Frascati (R-D), le Manuel d'Oslo (innovation) et le Manuel de Canberra (ressources humaines pour la science et la technologie), les deux derniers étant des publications conjointes avec 
Eurostat. Ces manuels sont le fruit des travaux conduits par l'OCDE et son Groupe d'experts nationaux pour conceptualiser les activités scientifiques et technologiques et développer des méthodes statistiques afin d'en mesurer les aspects les plus pertinents sur des bases concertées au niveau international.

Andrew Wyckoff Directeur par intérim pour la science, la technologie et l'industrie, OCDE
Fred Gault

Président du GENIST

à l'origine

de la première révision
Ward Ziarko

Président actuel du GENIST

Remerciements. La rédaction du Manuel de l'OCDE sur les statistiques des brevets a été confiée à Pluvia Zuniga et Dominique Guellec (OCDE), auxquels Hélène Dernis, Mosahid Khan, Teruo Okazaki et Colin Webb (OCDE) ont apporté leur concours. Une version préliminaire a été examinée par des organismes nationaux et internationaux actifs dans le secteur des brevets (Office européen des brevets, Japan Patent Office, United States Patent \& Trademark Office et Organisation mondiale de la propriété intellectuelle); par de grands utilisateurs de données de brevets (Eurostat, National Science Foundation des États-Unis et NISTEP du Japon); et par un groupe d'experts internationaux du domaine, notamment le professeur Akira Goto (Fair Trade Commission, Japon), le professeur Stuart Graham (Georgia Tech, États-Unis), Françoise Laville (Observatoire des sciences et techniques, France), Catalina Martinez (CSIC, Espagne), le professeur Ulrich Schmoch (FhG-ISI, Allemagne) et le professeur Jun Suzuki (Institute for Future Technology, Japon). 


\section{Table des matières}

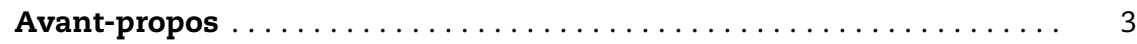

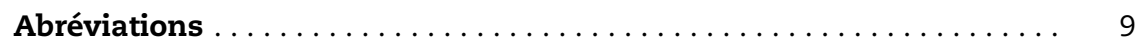

Chapitre 1 . Objectifs et portée du manuel. ............... 11

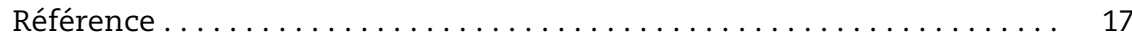

Chapitre 2. Les brevets comme indicateurs statistiques

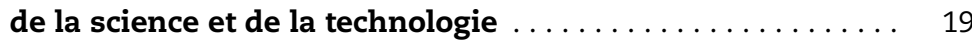

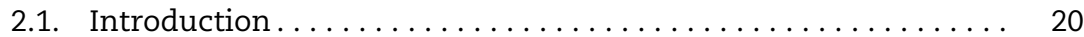

2.2. Les fondements juridiques des brevets . . . . . . . . . . 20

2.3. Voies administratives pour obtenir la protection .......... 21

2.4. Fondements économiques des brevets . . . . . . . . . 23

2.5. Informations contenues dans le document brevet . . . . . . 27

2.6. Les brevets comme indicateurs statistiques de l'activité d'invention............................ 29

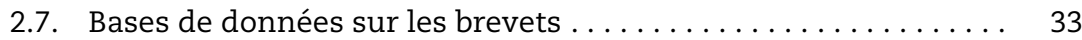

2.8. Thèmes de recherche $\ldots \ldots \ldots \ldots \ldots \ldots \ldots \ldots \ldots \ldots \ldots$

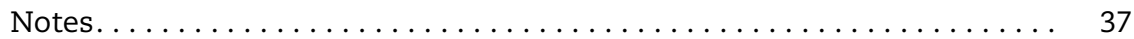

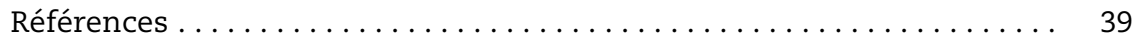

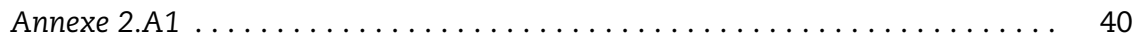

Chapitre 3 . Systèmes et procédures en matière de brevets. . . . . . . 45

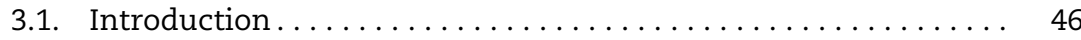

3.2. Procédure de base . . . . . . . . . . . . . . . . . . . 47

3.3. Procédures nationales et régionales. . . . . . . . . . . . . 54

3.4. Demande internationale de brevets............... 61

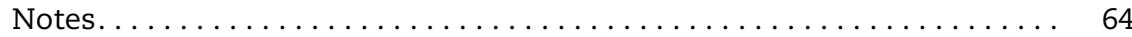

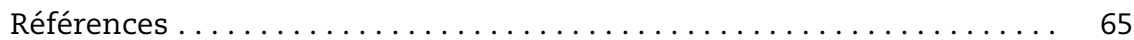

Chapitre 4. Critères de base pour la compilation d'indicateurs fondés

sur les brevets . . . . . . . . . . . . . . . . . . . . 67

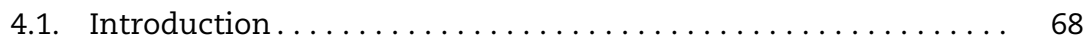

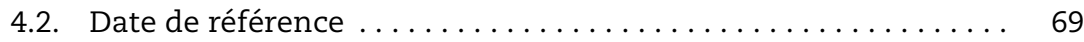

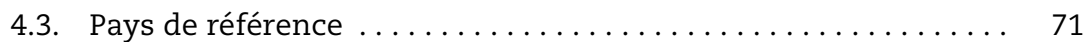

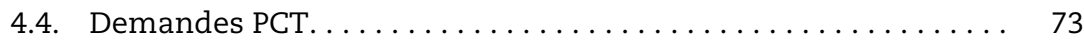

4.5. Familles de brevets . . . . . . . . . . . . . . . 80

4.6. Indicateurs fondés sur les brevets normalisés au niveau des pays . 84 


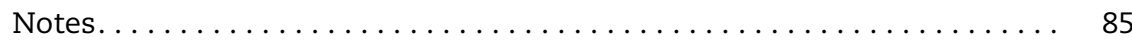

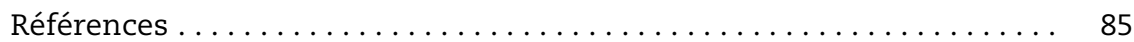

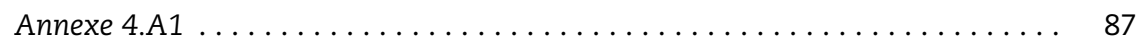

Chapitre 5 . Classification des brevets en fonction de différents critères . . 93

5.1. Introduction . . . . . . . . . . . . . . . . . . 94

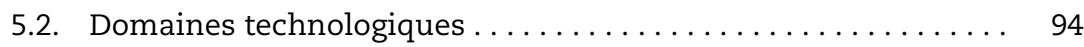

5.3. Classification des secteurs d'activité .................. 101

5.4. Classification régionale . . . . . . . . . . . . . . . . . . . . . 104

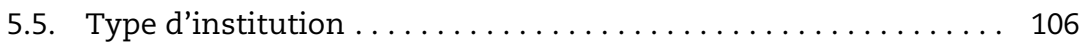

5.6. Brevets provenant d'entreprises commerciales . . . . . . . . . . . 108

5.7. Brevets par inventeurs . . . . . . . . . . . . . . . . . 110

Notes. . . . . . . . . . . . . . . . . . . . . . . . . . . . . 112

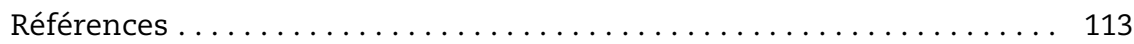

Chapitre 6. Utilisation et analyse des citations de brevets . . . . . . . . 117

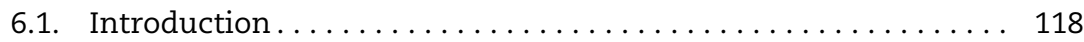

6.2. Qu'est-ce qu'une citation?.................... 118

6.3. Utilisations et applications des indicateurs de citations . . . . . 119

6.4. Pratiques des bureaux des brevets en matière de citation . . . . . 120

6.5. Les indicateurs fondés sur les citations. . . . . . . . . . . . . . 124

6.6. Littérature hors brevets (NPL) . . . . . . . . . . . . . . . . . 130

6.7. Autres indicateurs reposant sur les types de citations

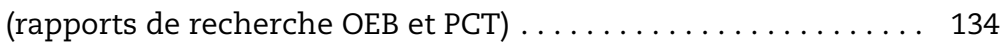

Notes....................................... 135

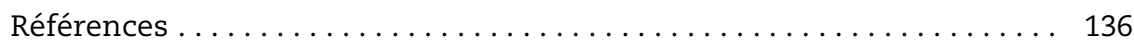

Chapitre 7. Indicateurs de l'internationalisation de la science

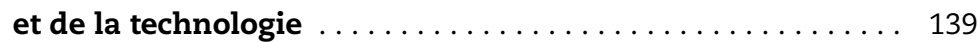

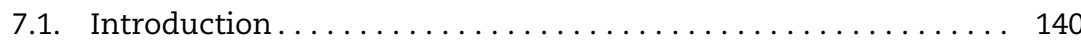

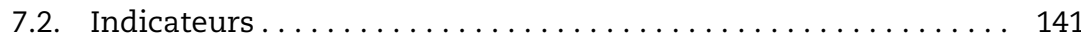

7.3. Propriété et stratégies de recherche. . . . . . . . . . . . . . 146

Notes. . . . . . . . . . . . . . . . . . . . . . . . . . . . . . . . 148

Références ................................ 148

Chapitre 8. Indicateurs de la valeur des brevets . . . . . . . . . . . . . 149

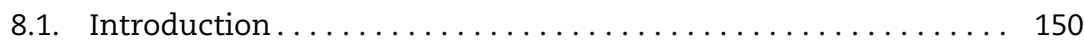

8.2. Citations en aval . . . . . . . . . . . . . . . . . 152

8.3. Indicateurs fondés sur les informations sur la procédure

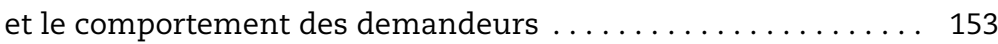

8.4. Autres indicateurs. . . . . . . . . . . . . . . . . . . . . . . . 160

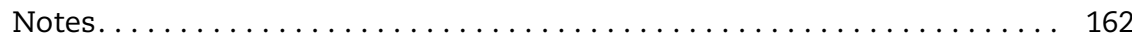

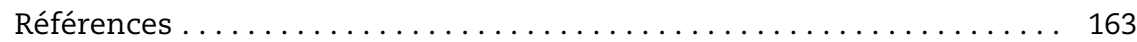

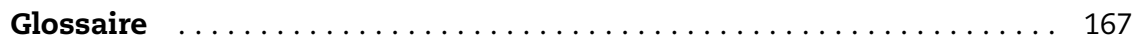




\section{Liste des encadrés}

1.1. Quelques exemples de statistiques de brevets publiées . . . . . . 16

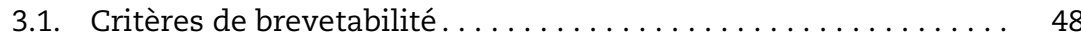

3.2. Principales dispositions de l'Accord ADPIC . . . . . . . . . 51

4.1. Méthodologies de rétropolation $\ldots \ldots \ldots \ldots \ldots \ldots \ldots \ldots \ldots$

4.2. Méthodes de rétropolation basées sur les transferts. . . . . . . . . 79

4.A1.1. Autres définitions des familles de brevets . . . . . . . . . 88

6.1. Le problème des brevets équivalents . . . . . . . . . . . . . . 123

7.1. Dispersion régionale des brevets . . . . . . . . . . . . 146

8.1. Réformes concernant la désignation des États. . . . . . . . . . . . 158

8.2. Un indicateur mixte (protection européenne) :

l'indice de la portée annuelle . . . . . . . . . . . . . . . . . . . . 159

\section{Liste des tableaux}

3.1. Différences entre les trois principaux bureaux des brevets . . . . 55

4.A1.1. Écarts dans les comptages de brevets (OEB) selon la référence

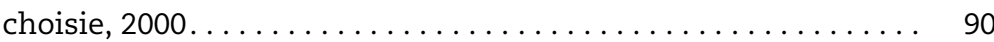

4.A1.2. Part des pays dans les demandes OEB selon divers critères d'attribution ........................... 91

5.1. Principales caractéristiques des codes CIB (exemple) . . . . . . . 97

5.2. Exemples de mots clés ou d'indices utilisés pour identifier le secteur de brevets . . . . . . . . . . . . . . . . . . . . 107

6.1. Occurrence des références brevets et hors brevets (USPTO - OEB) . 121

6.2. Occurrence des références aux revues scientifiques et aux autres sources dans les brevets USPTO et OEB . . . . . . . . 131

6.3. Occurrence des sources autres que les revues scientifiques dans les citations de brevets USPTO et OEB . . . . . . . . . . . . . . . . 131

6.4. Catégories de citations à l'OEB et selon le PCT . . . . . . . . . . . . 134

8.1. Principaux indicateurs de la valeur des brevets analysés dans les publications . . . . . . . . . . . . . . . . . 154

8.2. Part des pays dans le total des demandes de brevet en fonction de différents indicateurs . . . . . . . . . . . . . . . . 156

\section{Liste des graphiques}

2.A1.1. Page de garde d'une demande de brevet à l'OEB . . . . . . . . . 40

2.A1.2. Exemple de page de garde d'une demande de brevet au JPO . . . 41

2.A1.3. Page de garde d'une demande de brevet de l'USPTO. . . . . . . . 42

2.A1.4. Page de garde d'une demande PCT. . . . . . . . . . . . . 43

3.1. Chronologie des procédures PCT ................ 62

4.1. Part des pays dans les brevets déposés dans les trois grandes

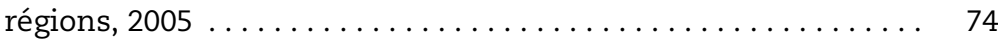

4.2. Brevets demandés selon la procédure PCT, désignations de l'OEB . . 75 
4.3. Part des pays dans les brevets demandés selon la procédure PCT,

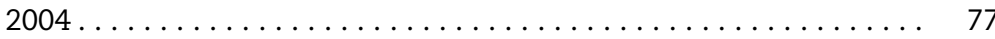

4.4. Part des demandes Euro-PCT entrant dans la phase régionale,

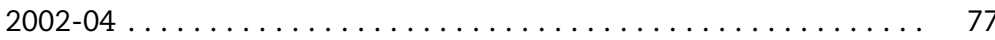

4.5. Part des pays dans le total des familles de brevets triadiques,

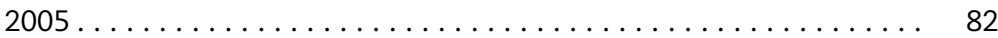

4.6. Exemple des familles de brevets au sens étroit et au sens large . . . 82

4.7. Familles de brevets triadiques rapportées au PIB, 2005 . . . . . . 83

4.8. Familles de brevets triadiques par millions d'habitants, 2005 . . 83

5.1. Évolution des dépôts de brevets sur les piles à combustible, part des brevets déposés selon le PCT, 1987-2004 . . . . . . . . 95

5.2. Part des pays dans les brevets sur les piles à combustible,

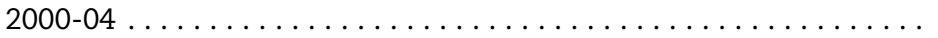

5.3. Parts des techniques apparentées dans les brevets sur les piles à combustible, $2000-04 \ldots \ldots \ldots \ldots \ldots \ldots \ldots \ldots \ldots$

5.4. Indice de spécialisation des brevets dans les biotechnologies déposés à l'OEB, 2000-02 . . . . . . . . . . . . . . . . 101

5.5. Brevets par branche et $\mathrm{R}-\mathrm{D}$ des entreprises dépôts selon le PCT, 2002-04 . . . . . . . . . . . . . . . . . . 104

5.6. Brevets ICT par région en Europe, aux États-Unis et au Japon. . . 106

6.1. Part de la littérature hors brevets dans les citations des rapports de recherche des demandes de brevets selon le PCT . . . . . . . . . . . . . . . . . . . . 133

6.2. Part du hors brevets dans les citations - tous brevets. . . . . . . 133

6.3. Part du hors brevets dans les citations - TIC . . . . . . . . . . 133

7.1. La mondialisation de la S-T. . . . . . . . . . . . . . . 143

7.2. Propriété étrangère des inventions nationales. . . . . . . . . . . . . . 144

7.3. Propriété nationale des inventions étrangères. . . . . . . . . . . . . 144

7.4. Composition de la propriété transnationale dans les demandes de brevet déposées 


\section{Abréviations}

ADPIC Accord sur les aspects des droits de propriété intellectuelle qui touchent au commerce (en anglais : TRIPS)

AFA Activité des filiales étrangères

ARIPO Organisation régionale africaine de la propriété industrielle

BEA Bureau of Economic Analysis (États-Unis)

CAFC Cour d'appel pour le circuit fédéral (États-Unis)

CBE

Convention sur le brevet européen (en anglais : EPC)

CIB

CIP

Classification internationale des brevets (en anglais : IPC)

CITI

Demande de continuation partielle

DPMA

Classification internationale type par industrie (en anglais : ISIC)

ECLA

Deutsches Patent- und Markenamt (Allemagne)

EPLA

Classification européenne des brevets

Accord sur le règlement des litiges en matière de brevets européens

FhG-ISI Fraunhofer Institute for Systems and Innovation Research

GATT Accord général sur les tarifs douaniers et le commerce

IIP

Institute of Intellectual Property (Japon)

INID Identification numérique internationale des données

bibliographiques

INPI Institut national de la propriété intellectuelle (France)

IPRP Rapport préliminaire international sur la brevetabilité

ISA Administrations chargées de la recherche internationale

ISR Rapport de recherche internationale

NACE Nomenclature statistique des activités économiques

dans la Communauté européenne

NBER National Bureau of Economic Research (États-Unis)

NISTEP National Institute of Science and Technology Policy (Japon)

NSF National Science Foundation (États-Unis)

NUTS Nomenclature des unités territoriales statistiques

OCDE Organisation de coopération et de développement économiques

OEB Office européen des brevets

OMC Organisation mondiale du commerce (en anglais : WTO)

OMPI Organisation mondiale de la propriété intellectuelle

OPIC Office de la propriété intellectuelle du Canada (en anglais : CIPO) 


$\begin{array}{ll}\text { OST } & \text { Observatoire des sciences et des techniques (France) } \\ \text { PATSTAT } & \text { Worldwide Statistical Patent Database (OEB) } \\ \text { PCT } & \text { Traité de coopération en matière de brevets } \\ \text { PME } & \text { Petites et moyennes entreprises } \\ \text { SCIAN } & \text { Système de classification des industries de l'Amérique du Nord } \\ & \text { (en anglais : NAICS) } \\ \text { SIC } & \text { Classification type par industrie } \\ \text { SIPO } & \text { Office d'État de la propriété intellectuelle de la République } \\ & \text { populaire de Chine } \\ \text { STAN } & \text { Base de données pour l'analyse structurelle } \\ \text { TIC } & \text { Technologies de l'information et de la communication } \\ \text { TL } & \text { Niveau territorial } \\ \text { UE } & \text { Union européenne } \\ \text { USPC } & \text { Classification US des brevets } \\ \text { USPTO } & \text { United States Patent and Trademark Office } \\ \text { WOISA } & \text { Written opinion of the international search authorities } \\ & \text { (Avis écrit de l'administration chargée de l'examen préliminaire } \\ & \text { international) }\end{array}$


Chapitre 1

\section{Objectifs et portée du manuel}




\section{L'}

objet de ce manuel est de fournir des informations de base sur les données relatives aux brevets utilisés pour la mesure de la science et de la technologie (S-T), sur la construction d'indicateurs de l'activité technologique et des lignes directrices pour la compilation et l'interprétation d'indicateurs utilisant les brevets dans ce contexte.

Parallèlement à d'autres indicateurs de la science et de la technologie comme les dépenses et le personnel de R-D, les données des enquêtes sur l'innovation ou d'autres sources, les brevets représentent une source d'information d'un grand intérêt sur l'activité d'invention. Les données sur les brevets sont complémentaires des autres données scientifiques et technologiques. Il est généralement utile d'utiliser conjointement plusieurs types de données (R-D, innovation, brevets) pour bénéficier d'une validation croisée et faciliter l'interprétation. Tous ces indicateurs ont leurs points forts et leurs points faibles; ils retracent également différentes phases du processus d'innovation. Le présent manuel appartient à la famille des Manuels de «Frascati » de l'OCDE, les autres étant le Manuel de Frascati sur la R-D, le Manuel d'Oslo sur l'innovation, le Manuel de la balance des paiements technologiques (BPT) et le Manuel de Canberra sur les ressources humaines.

Les statistiques sur les brevets servent depuis longtemps à évaluer l'activité scientifique et technologique. Des travaux ayant eu un large retentissement ont été réalisés dans les années 50 par Jakob Schmookler, un universitaire américain, qui a utilisé le nombre de brevets comme indicateurs du changement technologique dans des branches spécifiques. L'utilisation des données sur les brevets s'est développée en parallèle avec la puissance des ordinateurs. L'OCDE a tenu en 1985 une conférence sur les nouveaux indicateurs de la science et de la technologie, au cours de laquelle plusieurs présentations ont été consacrées aux statistiques des brevets. Sur cette période, les publications scientifiques et technologiques ont été de plus en plus nombreuses à comporter une section sur les indicateurs de brevets. En écho à cette utilisation croissante des données sur les brevets par les universitaires, le célèbre universitaire Zvi Griliches a publié en 1990 un article devenu classique qui évaluait les façons d'utiliser ces données. L'OCDE a publié son premier manuel des brevets en 1994. À la même époque, les bureaux des brevets augmentaient leurs efforts pour retracer l'activité de protection par brevet en s'appuyant sur des statistiques poussées. Plusieurs entreprises ont prospéré dans la vente de renseignements commerciaux tirés des brevets, largement fondés sur une approche statistique. 
Les données sont devenues de plus en plus disponibles sous forme électronique et la base de données des statistiques mondiales de brevets (PATSTAT), qui rassemble des données de la quasi-totalité des bureaux des brevets mondiaux selon une présentation normalisée, a été publiée en 2006 par l'Office européen des brevets (OEB).

Les brevets sont un moyen de protéger les inventions réalisées par les entreprises, les institutions ou les particuliers, et à ce titre les données de brevets peuvent être interprétées comme un indicateur de l'activité inventive. Avant qu'une invention puisse devenir une innovation, des activités entrepreneuriales complémentaires sont nécessaires pour la développer, la concrétiser et la mettre sur le marché. Les indicateurs de brevets donnent des informations sur la production et les processus des activités d'invention. Les brevets protègent les inventions et, bien que la relation ne soit pas simple, de nombreuses recherches ont montré qu'une fois mises en place des méthodes de contrôle appropriées, il existe une relation positive entre les comptages de brevets et d'autres indicateurs liés aux performances inventives (productivité, parts de marché, etc.). La relation peut varier selon les pays, les branches ou l'époque. Grâce à l'information fournie dans le document brevet, l'exploitation statistique de ces données apporte un éclairage unique sur les processus d'invention. Les brevets donnent des informations sur le contenu technologique de l'invention (notamment ses domaines techniques spécifiques) et sur la localisation géographique du processus d'invention. Dans la mesure où ils identifient le propriétaire et les inventeurs, par rapprochement avec d'autres données, les brevets peuvent illustrer l'organisation du processus de recherche sous-jacent (par exemple, les alliances entre entreprises ou entre entreprises et organismes publics de recherche, le rôle respectif des multinationales et des petites entreprises, la taille et la composition des équipes de recherche, etc.). Les brevets peuvent également donner des informations sur la mobilité et les réseaux des inventeurs, et permettre de suivre la diffusion des connaissances (l'influence de telle ou telle invention sur d'autres, ou les inventions ultérieures).

Un autre avantage des données sur les brevets est qu'elles sont largement disponibles pour un coût relativement modique. Il s'agit de données à vocation administrative. Des bases de données sur les brevets sont compilées par les bureaux des brevets à des fins internes, pour gérer leurs procédures administratives d'examen des demandes et surtout pour remplir leur mission de diffusion de l'information. Ces bases sont disponibles sur Internet en consultation publique. Leur adaptation à des fins statistiques nécessite quelques investissements supplémentaires, mais ces bases de données sont désormais assez largement disponibles. La baisse des coûts informatiques facilite l'exploitation de ces données à grande échelle et de façon décentralisée et ouverte. Aucune règle de confidentialité n'interdit l'accès aux informations publiées sur les brevets, bien que la publication intervienne généralement 
18 mois après la demande initiale. De ce fait, des données sur les brevets sont publiquement disponibles dans la plupart des pays du monde, souvent sur de longues séries chronologiques.

Les indicateurs utilisant les brevets ont également des inconvénients, et ils doivent donc être utilisés et interprétés avec prudence. Toutes les inventions ne sont pas brevetées. Les entreprises peuvent préférer le secret, ou s'en remettre à d'autres mécanismes, pour dominer leur marché. L'activité de brevetage diffère selon les branches et les pays, ainsi que selon l'époque. La répartition des brevets en fonction de leur valeur est connue pour être biaisée, car seul un petit nombre a une très forte valeur technique et économique tandis que beaucoup ne seront en définitive jamais exploités. Les simples comptages, qui accordent le même poids à tous les brevets, indépendamment de leur valeur, peuvent donc être trompeurs, notamment sur les petits échantillons. Les différences de normes selon les bureaux des brevets et la période peuvent influer sur les comptages de brevets, même si l'activité inventive sous-jacente peut rester la même.

Les données sur les brevets sont complexes. Il faut avoir une connaissance précise des législations et procédures sur le brevetage et sur le comportement des entreprises dans ce domaine de manière à appliquer les mesures de contrôle et les filtres appropriés sur les données pour obtenir des indicateurs significatifs et interpréter ceux-ci correctement. La complexité des données de brevets s'explique par plusieurs facteurs, notamment la diversité des bureaux des brevets et des procédures (la juridiction pouvant être nationale ou régionale), la disponibilité de différentes voies pour obtenir la protection par brevet (nationale ou internationale) et l'évolution des comportements des demandeurs à cet égard, et les différences de statuts et de dates dans les documents relatifs au brevet du fait de la complexité des procédures (demande, délivrance, phase internationale, etc.). De plus, certaines informations en relation avec les brevets peuvent encore faire l'objet de débats entre experts (indicateurs de valeur, nombre de citations/revendications, etc.).

Avec l'essor des statistiques fondées sur les brevets, il est nécessaire de partager les connaissances sur la façon d'utiliser les données et d'élaborer des normes qui amélioreront la qualité des indicateurs et réduiront les possibilités d'erreur d'interprétation. Ainsi, il est encore fréquent que les analystes comparent les comptages de brevets provenant de différents bureaux des brevets pour évaluer les performances des pays, alors que ces comptages ne sont généralement pas directement comparables. Le présent manuel : i) présente les informations de base nécessaires pour comprendre ou compiler des statistiques basées sur les brevets; et ii) propose des normes (formules utilisables pour les indicateurs et terminologie) pour la compilation d'indicateurs sur les brevets. Toutefois, des normes ne sont proposées que dans les domaines où un certain consensus s'est dégagé parmi les experts. Dans certains domaines, 
notamment les plus récents, aucun consensus de ce type n'est encore apparu, et le présent manuel mentionnera donc à la place les diverses options. Le manuel s'adresse : i) aux utilisateurs et compilateurs de statistiques sur les brevets dans les organismes statistiques et agences en charge de la science et de la technologie; et ii) aux utilisateurs de bases de données sur les brevets qui effectuent des travaux analytiques sur la dynamique de la technologie au niveau de l'entreprise, de la région ou du pays.

Le présent manuel est une version révisée de l'édition 1994 du manuel de l'OCDE (La mesure des activités scientifiques et technologiques : les données sur les brevets d'invention et leur utilisation comme indicateurs de la science et de la technologie), qui marquait une première étape dans le processus de clarification et d'harmonisation des indicateurs fondés sur les brevets. Cette publication décrivait le contexte juridique et économique des brevets - étape nécessaire avant l'élaboration de statistiques - et répertoriait les indicateurs qui pourraient être construits à partir des bases de données sur les brevets. Elle répertoriait également un certain nombre de problèmes méthodologiques rencontrés lors du calcul d'indicateurs fondés sur les brevets. Depuis 1994, l'expérience dans l'utilisation de données sur les brevets s'est considérablement enrichie, et la présente version révisée du manuel ambitionne de tirer parti de cette expérience. Le manuel s'appuie en particulier, mais pas exclusivement, sur l'expérience de l'OCDE et des membres du Groupe d'étude sur les statistiques de brevets dans l'élaboration de normes statistiques pour la compilation d'indicateurs des brevets destinés à mesurer l'activité d'invention. Les membres de ce groupe d'étude sont l'Office européen des brevets (OEB), le Japan Patent Office (JPO), le Patent and Trademark Office des États-Unis (USPTO), l'Organisation mondiale de la propriété intellectuelle (OMPI), Eurostat et la National Science Foundation (NSF) des États-Unis.

Aujourd'hui, la plupart, sinon la totalité, des études statistiques sur la science et la technologie au niveau national et international comportent une section sur les brevets (voir encadré 1.1). Parallèlement, un nombre croissant de rapports d'orientation utilisent des données sur les brevets pour suivre les évolutions dans des domaines techniques ou institutionnels particuliers. Un nouveau domaine de recherche universitaire est apparu qui exploite les données sur les brevets.

Le présent manuel est architecturé comme suit. Le chapitre 2 traite de la signification des indicateurs sur les brevets : fondements juridiques, dimension économique, informations contenues dans le document brevet et nature des questions analytiques auxquelles les indicateurs de brevets peuvent apporter des réponses. Le chapitre 3 détaille les procédures de prise de brevet, en mettant plus particulièrement l'accent sur l'Europe, le Japon, les États-Unis et la procédure internationale (Traité de coopération en matière de brevets). Le chapitre 4 rend compte des règles générales qui s'appliquent lors de la 


\section{Encadré 1.1. Quelques exemples de statistiques de brevets publiées}

\section{Publications scientifiques et technologiques}

États-Unis (2006) : Science and Engineering Indicators (National Science Foundation).

Brevets délivrés à des inventeurs des États-Unis et étrangers par pays/économie d'origine.

Principales entreprises déposant des brevets.

Japon (2004) : Science and Technology Indicators (National Institute of Science and Technology Policy - NISTEP).

Nombre de demandes de brevets nationales et étrangères déposées dans certains bureaux des brevets nationaux.

Eurostat (2007) : Statistiques en bref, ouvrages statistiques et ouvrages de poche sur la science, la technologie et l'innovation en Europe.

Brevets OEB et USPTO, par pays, par régions.

Familles de brevets triadiques.

OCDE (2007) : Compendium statistique sur les brevets.

Familles de brevets triadiques, brevets sur les TIC, la biotechnologie et les nanotechnologies (Traité de coopération en matière de brevets - PCT).

Propriétés transfrontières d'inventions, co-inventions transfrontières en matière de brevets.

France (2006) : Observatoire des sciences et techniques, Indicateurs de sciences et technologies. Finlande (2007) : Patenting, Statistics Finland.

\section{Bureaux de brevets et organisations apparentées}

Statistiques de l'OMPI (2006) : Rapport concernant les données statistiques du PCT.

Demandes internationales PCT (par origine, langue de dépôt, domaine technique).

Demandes internationales PCT par bureau récepteur.

Rapport statistique trilatéral (annuel) : OEB, JPO et USPTO.

Activité de brevetage par blocs : demandes initiales, origine et cible des demandes, délivrances.

Activité interbloc : courants des demandes, familles de brevets.

Dépôt de demandes PCT, par année de dépôt, familles de brevet trilatérales.

Office européen des brevets : Rapport annuel.

Japan Patent Office : Rapport annuel.

US Patent and Trademark Office : Rapport annuel et Patent Statistics Reports.

compilation d'indicateurs sur les brevets : date de référence, pays de référence, demandes de brevet internationales/nationales et famille de brevets. Le chapitre 5 décrit les diverses classifications qui peuvent s'appliquer aux brevets : domaine technique, branche, secteur industriel et région, et les méthodes d'examen pour l'attribution des brevets à des entreprises ou des inventeurs particuliers. Le chapitre 6 est consacré aux citations de brevets : leur signification et leur utilisation dans les indicateurs. Le chapitre 7 passe en 
revue les indicateurs de l'internationalisation des activités scientifiques et technologiques fondés sur les brevets. Le chapitre 8 analyse les indicateurs de la valeur des brevets, tels que maintien en vigueur, taille de la famille, nombre de classes techniques, etc.

\section{Référence}

Griliches, Z. (1990), « Patent Statistics as Economic Indicators: A Survey », Journal of Economic Literature, 28, pp.1661-1707. 

Chapitre 2

Les brevets comme indicateurs statistiques de la science et de la technologie 


\subsection{Introduction}

Les propriétés statistiques des données sur les brevets sont déterminées par leurs caractéristiques juridiques et par leur mise en œuvre économique car celles-ci conditionnent la nature des inventions qui sont protégées, l'identité du propriétaire et les informations qui sont communiquées (et donc rendues accessibles aux statisticiens), de même que l'importance que revêtent les brevets pour l'industrie, etc. On trouvera dans ce chapitre un panorama des fondements juridiques et économiques des brevets. Celui-ci décrit les concepts de base nécessaires pour utiliser les brevets comme indicateurs de la science et de la technologie (S-T).

\subsection{Les fondements juridiques des brevets}

Les brevets sont des instruments juridiques utilisés dans la vie économique. Un brevet est un titre juridique protégeant une invention (article 28 de l'accord sur les aspects des droits de propriété intellectuelle qui touchent au commerce (ADPIC) :

«I. Un brevet conférera à son titulaire les droits exclusifs suivants :

a) dans les cas où l'objet du brevet est un produit, empêcher des tiers agissant sans son consentement d'accomplir les actes ci-après : fabriquer, utiliser, offrir à la vente, vendre ou importer à ces fins ce produit ;

b) dans les cas où l'objet du brevet est un procédé, empêcher des tiers agissant sans son consentement d'accomplir l'acte consistant à utiliser le procédé et les actes ci-après : utiliser, offrir à la vente, vendre ou importer à ces fins, au moins le produit obtenu directement par ce procédé.

II. Le titulaire d'un brevet aura ainsi le droit de céder ou de transmettre par voie successorale le brevet et de conclure des contrats de licence. "

Le brevet confère à son titulaire un ensemble de droits d'exclusivité sur une invention (produit ou procédé qui est nouveau, qui implique une activité d'invention et est susceptible d'applications industrielles) telle que définie dans les "revendications ". La protection juridique conférée par le brevet donne à son titulaire le droit d'exclure autrui de la fabrication, de l'utilisation, de la vente, de l'offre à la vente ou de l'importation de l'invention brevetée pendant la durée du brevet, qui est généralement de vingt ans à compter de la date de demande, et dans le ou les pays visés par la protection. Cet ensemble de droits donne au titulaire du brevet un avantage concurrentiel. Les brevets 
peuvent également faire l'objet de contrats de licence ou être utilisés pour aider à créer ou financer une entreprise. Il est donc possible d'en extraire de la valeur même si le propriétaire ne dispose pas de capacités de fabrication propres (cas par exemple des universités).

Les brevets confèrent des droits temporaires, valables pour une durée maximale de 20 ans à compter de la date de demande, après quoi l'invention qu'ils protègent tombe dans le domaine public ${ }^{1}$. Les brevets sont des droits territoriaux; ils ne s'appliquent qu'au pays pour lequel ils ont été accordés. Ainsi, un brevet accordé aux États-Unis ne conférera pas d'exclusivité au Japon - il empêchera seulement le brevetage de la même invention au Japon (dans la mesure où pour obtenir un brevet, il faut un caractère novateur à l'échelle mondiale). Des brevets sont accordés pour des inventions touchant tous les domaines de la technologie. De façon générale, les lois de la nature, les phénomènes naturels et les idées abstraites ne sont pas brevetables (il existe bien entendu un débat sur les limites du système - ainsi, un logiciel estil une « idée abstraite » ou une invention brevetable?).

\subsection{Voies administratives pour obtenir la protection}

Les brevets s'obtiennent à la suite des procédures administratives spécifiques $^{2}$. Pour obtenir un brevet, l'inventeur doit présenter une demande auprès d'un bureau des brevets qui vérifiera que l'invention remplit les critères juridiques applicables puis, selon les cas, accordera le brevet ou rejettera la demande. Il existe différentes « voies » que peuvent emprunter les inventeurs pour obtenir un brevet, leur choix étant fonction de leur stratégie commerciale nationale ou mondiale.

- Voie nationale. Quand un inventeur (particulier, entreprise, organisme public, université, organisation sans but lucratif) décide de protéger une invention, la première étape consiste à déposer une demande auprès d'un bureau national de brevets (en général le bureau national du pays du demandeur). La première demande déposée dans le monde (dans n'importe quel bureau des brevets) pour une invention donnée est appelée " demande prioritaire " à laquelle est associée une "date de priorité ". Le bureau des brevets commence alors une procédure de recherche et d'examen pour voir si le brevet peut ou non être accordé, c'est-à-dire si l'invention concerne un domaine brevetable, a un caractère de nouveauté et d'inventivité («nonévidence pour une personne du métier de compétence moyenne ") et peut faire l'objet d'applications industrielles. La demande est généralement publiée 18 mois après son dépôt (date de publication). Le délai entre la demande et la délivrance ou le refus du brevet n'est pas fixe; il peut varier entre deux et huit ans, avec des écarts sensibles selon les bureaux des brevets. 
- Voie internationale. Depuis 1883, date à laquelle les procédures ont été normalisées en vertu de la Convention de Paris (quelque 170 pays signataires en 2006), les demandeurs qui souhaitent protéger leurs inventions dans plus d'un pays ont 12 mois à compter de la date de priorité pour déposer des demandes dans d'autres pays parties à la Convention, et s'ils le font, la protection s'applique à partir de la date de priorité dans les pays concernés. Alternativement, les inventeurs peuvent utiliser la procédure PCT (Traité de coopération en matière de brevets), qui est en vigueur depuis 1978 et est administrée par l'Organisation mondiale de la propriété intellectuelle (OMPI). Sous la procédure PCT il est possible de retarder de manière significative les procédures nationales ou régionales (jusqu'à la fin du trentième mois depuis la date de priorité) au moyen d'une procédure unifiée de dépôt (voir le chapitre 3). Les demandeurs ont donc davantage de temps pour remplir les conditions nationales et peuvent utiliser le temps pour évaluer les chances d'obtenir des brevets et d'exploiter l'invention (estimer la concurrence, trouver des partenaires avec licence, etc.) ${ }^{3}$. C'est maintenant la voie la plus populaire parmi les inventeurs visant les marchés mondiaux.

- Voies régionales. Les demandeurs peuvent aussi soumettre une demande de brevet auprès d'un bureau régional (par exemple, bureau eurasien, OAPI). Ainsi, l'OEB (Office européen des brevets) est un bureau régional qui recherche et examine les demandes de brevets pour les pays européens, et qui comptait 32 membres en 2007. L'OEB délivre des " brevets européens ", qui sont valables dans l'ensemble de ses États membres auprès desquels le détenteur a validé ses droits. La validation nécessite la traduction dans la langue nationale et le paiement des taxes nationales. À ce stade national, les brevets européens sont soumis aux législations nationales.

Les législations nationales en matière de brevet doivent respecter les normes internationales désormais définies dans l'ADPIC (Accord sur les aspects des droits de propriété intellectuelle qui touchent au commerce) qui est un traité international faisant partie du paquet OMC (Organisation mondiale du commerce) signé en 1994. Sous réserve que le pays soit membre de l'OMC, l'ADPIC impose des conditions strictes aux membres de l'OMC, comme par exemple la brevetabilité des inventions dans tous les domaines technologiques, une durée minimale de protection des brevets de 20 ans, des limitations quant à la protection obligatoire par brevet, etc.

Une fois délivré par une autorité administrative, le brevet peut toujours être contesté par des tiers. Ceux-ci peuvent emprunter la voie juridique, en demandant que le brevet soit révoqué ou jugé invalide. Le titulaire du brevet doit également aller devant un tribunal pour faire appliquer son brevet en faisant valoir sa violation par des tiers. Il s'agit là encore d'une procédure strictement nationale, même en Europe ${ }^{4}$. 
La procédure d'obtention d'un brevet implique la communication d'un grand nombre d'informations à des fins juridiques ou administratives. Cette information peut présenter un très grand intérêt pour les statisticiens. On trouvera dans les graphiques 2.A1.1 à 2.A1.4 la reproduction de la page de garde d'un dépôt de brevet publié par l'OMPI, l'OEB, le JPO et l'USPTO. Parmi les informations figurant dans le document brevets utiles pour les statisticiens, on peut citer :

- Le numéro de dépôt et le type de demande, le numéro de publication, etc. ${ }^{5}$.

- Le nom et l'adresse de l'inventeur; le nom et l'adresse du demandeur ou du bénéficiaire (généralement l'entreprise employant l'inventeur).

- Des détails techniques concernant l'invention : titre, résumé, description détaillée de l'invention, indications sur la façon dont elle est construite, la façon dont elle s'utilise et sur les bénéfices qu'elle apporte par rapport à ce qui existe déjà.

- Une liste des revendications, qui sont une définition claire et concise de ce que le brevet protège juridiquement.

- Les classes techniques auxquelles le brevet appartient.

- Une série de dates : date de priorité, de demande, de délivrance, etc.

- Une liste de références à d'autres brevets ou à la littérature hors brevets (par exemple, publications scientifiques) considérées comme pertinentes pour déterminer la brevetabilité de l'invention.

\subsection{Fondements économiques des brevets}

Le but revendiqué du système de brevet est d'encourager l'invention et les progrès techniques en assurant une période temporaire d'exclusivité sur l'invention en échange de sa divulgation. En assurant protection et exclusivité, le brevet est un outil servant à encourager les inventeurs à investir dans la recherche et dans les travaux postérieurs d'innovation qui conduiront à l'exploitation pratique des inventions.

Le brevet renforce l'inventivité de différentes manières (Scotchmer, 2004; Guellec et van Pottelsberghe, 2007). Du fait que les brevets révèlent des connaissances nouvelles à travers la divulgation des inventions, ils diffusent des informations qui sinon seraient gardées secrètes, permettant ainsi à d'autres inventeurs de développer de nouvelles inventions. En diffusant l'information sur les inventions qui ont été réalisées et sont protégées, le système de brevets décourage également la duplication inutile des efforts de recherchedéveloppement (R-D), en incitant les chercheurs à se concentrer sur les domaines véritablement nouveaux. De plus, les brevets étant des titres légaux, ils peuvent faire l'objet de transactions. Les brevets facilitent donc le développement des marchés de technologie, ce qui améliore l'allocation des ressources (pour 
l'utilisation de la technologie) au sein de l'économie, et ils permettent aux utilisateurs les plus efficients de mettre en œuvre des inventions (par exemple à travers des licences) même s'ils n'en sont pas nécessairement les auteurs, ou d'échanger les technologies nécessaires à de nouvelles inventions.

Il faut un cadre juridique pour protéger les inventions, car l'information est un bien public non excluable et non rival. L'expression " non excluable » signifie qu'il est impossible d'interdire à quelqu'un qui n'a pas supporté le coût de l'invention, d'utiliser le bien (notion de " passager clandestin "). Un bien "non rival " est un bien dont la consommation par une personne ne réduit pas la quantité disponible pour autrui (c'est-à-dire que le coût marginal est nul). Les brevets rendent l'invention excluable, car il faut l'autorisation de l'inventeur pour pouvoir l'exploiter, tout en conservant son caractère non rival, de sorte que plusieurs entités peuvent l'exploiter simultanément.

Toutefois, l'information (connaissance) n'est pas un bien public parfait et elle peut être protégée par d'autres moyens que les brevets ou, le plus souvent, de façon complémentaire au brevet (Blind et al., 2002). D'autres stratégies sont ainsi utilisables pour protéger la rentabilité de l'invention comme le secret ${ }^{6}$, l'avance sur le marché et le raccourcissement des cycles des produits, la pratique de prix faibles ou d'autres approches concurrentielles (moyens de production et de marketing; service après-vente, contrats à long terme). Le recours à ces stratégies a été confirmé par diverses enquêtes auprès des entreprises (Levin et al., 1987; Cohen et al., 2000). Ainsi, dans l'enquête Carnegie Mellon (1994) auprès d'entreprises américaines, il a été constaté que le secret et l'avance sur les concurrents étaient considérés comme les deux mécanismes d'appropriabilité les plus efficaces pour les innovations de produits, avec des notations un peu supérieures à $50 \%$ pour chacune. De plus, les entreprises déclaraient que des demandes de brevets n'étaient présentées que pour $52 \%$ des inventions de produits et $33 \%$ des inventions de procédés. Dans l'enquête NISTEP (Goto et Nagata, 1997) auprès des entreprises japonaises, il a été constaté que l'avance sur les concurrents (41\%) était également classée comme le mécanisme d'appropriabilité le plus efficace pour les innovations de produits, et que l'exploitation d'actifs complémentaires pour la fabrication (33\%) venait immédiatement après la protection par brevet (38\%). Dans une enquête réalisée sur des demandeurs auprès de l'OEB en 2006, il est observé qu'environ $50 \%$ des inventions débouchent sur un brevet, les taux les plus forts étant observés pour l'audio, la vidéo et les médias ainsi que pour l'électronique (environ 70 \%). Les taux les plus faibles concernaient la biotechnologie et la chimie organique pure et appliquée, avec environ $25 \%$.

Les brevets présentent un arbitrage. S'ils encouragent les inventions ex ante, ils ont un coût ex post. En donnant l'exclusivité d'exploitation à une entreprise particulière, un brevet limite la concurrence et fait monter les prix, excluant ainsi des clients qui auraient été disposés à acquitter le coût marginal d'un bien mais 
ne peuvent payer le surcoût demandé par le propriétaire du brevet. Cela est considéré comme le principal dilemme soulevé par les brevets; ceux-ci peuvent améliorer l'efficience dynamique de l'économie (en encourageant l'innovation, et donc la croissance et la création de valeurs), mais cela se fait au détriment de l'efficience statique (moindre concurrence et donc prix plus élevés, qui excluent certains consommateurs) ${ }^{7}$. La politique en matière de brevets fournit divers outils pour remédier à ce dilemme. En particulier, aussi bien la durée de la protection par brevet que le champ de la couverture assurée (importance de la différence que doit présenter un autre produit pour ne pas être en violation du brevet) sont essentiels dans l'équilibre entre protection et diffusion : des brevets offrant une couverture plus longue et plus large favorisent la protection, alors que les brevets de durée et de portée plus courtes encouragent la diffusion.

La politique est plus difficile à élaborer dans le cas des inventions cumulatives (ou complémentaires, c'est-à-dire des inventions qui s'appuient les unes sur les autres). Dans de telles circonstances, certains font valoir que les brevets peuvent limiter l'accès à des technologies qui sont nécessaires pour poursuivre l'innovation, dans la mesure où les innovateurs suivants ne doivent pas violer des connaissances brevetées alors qu'ils en ont besoin pour leurs propres inventions. Cette configuration d'inventions cumulatives soulève la question de savoir comment concilier la protection concédée à l'invention initiale et les inventions qui en découlent. Ce dilemme existe par exemple en biotechnologie, concernant des traitements particuliers (brevetés) associés à certaines voies génétiques (également brevetées). Dans le cas d'inventions nouvelles reposant sur plusieurs inventions déjà brevetées, comme cela se passe par exemple en biotechnologie et dans le logiciel, le nouvel inventeur doit négocier l'accès à chacune des inventions existantes. Dans de telles circonstances, les transactions peuvent être tellement coûteuses qu'elles sont susceptibles d'empêcher la nouvelle invention. Il existe certaines solutions fondées sur les brevets pour réduire les coûts de transaction, telles que les pools de brevets (des consortiums d'entreprises décidant de se céder mutuellement leurs brevets et de céder des licences à des tiers), et des centres d'échange de brevets qui visent à normaliser les transactions (s'agissant des clauses contractuelles, des montants des redevances, etc.). Toutefois, pour assurer la conformité avec les droits conférés par les brevets et l'efficacité du marché, les politiques en matière de brevets doivent se conformer aux politiques relatives à la concurrence et à la législation antitrust ${ }^{8}$.

En raison de ces avantages et inconvénients dans l'utilisation des brevets comme instruments de politique, le débat se poursuit entre les économistes sur la meilleure façon de concevoir un système de brevets et sur la question de savoir s'il est tout simplement dans l'intérêt de la collectivité d'établir un tel système. 
Aucun consensus absolu ne s'est dégagé, mais il existe un large accord sur les points suivants :

- Les brevets accordés devraient être de « haute qualité », ce qui signifie qu'ils ne devraient couvrir que les inventions importantes, et révéler le contenu effectif de l'invention .

- La politique de concurrence devrait maintenir un lien étroit sur le système de brevet.

- Le système de brevet devrait être utilisé en complément d'autres instruments d'action de la politique d'innovation, notamment la politique scientifique, les politiques sectorielles et les politiques en matière de marché public.

- Les mécanismes qui facilitent la circulation des brevets et l'accès à ces derniers devraient être encouragés, mais ils ne devraient pas nuire à la concurrence (par exemple, pool de brevets, contrats de cession de licences, etc.).

Depuis le début des années 80, l'émergence d'importants changements au niveau des marchés et des politiques a contribué à élargir le rôle des brevets dans l'économie. Avec l'augmentation de la concurrence internationale, l'émergence des technologies de l'information et de la biotechnologie et l'importance croissante des entreprises nouvelles et des entreprises spécialisées dans la R-D, l'utilisation des brevets s'est développée parmi les entreprises innovantes. Le rôle croissant de la concurrence technologique sur les marchés a donné davantage d'importance aux droits de propriété intellectuelle dans la valeur économique des entreprises. Parallèlement, depuis le début des années 80 , la politique en matière de brevets à l'échelle mondiale évolue vers le renforcement des droits des détenteurs de brevets. Aux États-Unis, le Federal Court Improvements Act, promulgué en mars 1982, a créé la Court of Appeals of the Federal Circuit (CAFC) qui centralise les décisions concernant les brevets (la CAFC s'est vu confier la juridiction sur les appels des affaires de brevets dans l'ensemble des circuits fédéraux); par ailleurs, depuis 1980, le Bayh-Dole Act permet à des groupes de recherche sans but lucratif de breveter et commercialiser des technologies mises au point avec des crédits fédéraux, en vue de faciliter leur commercialisation.

En Europe, la création de l'Office européen des brevets (établi en 1977) s'est traduite par un renforcement de la protection par brevet dans de nombreux pays. Au Japon, une série de réformes depuis la fin des années 90 a eu tendance à renforcer les droits des titulaires de brevets. La signature des ADPIC en 1994 a marqué la volonté des pays d'imposer une meilleure harmonisation des droits en matière de brevet à l'échelle mondiale. À la suite de ces évolutions, le nombre des demandes de brevets a augmenté considérablement dans le monde entre le milieu des années 90 et le milieu des années 2000 et il continue de croître. Ainsi, le nombre des demandes de brevets auprès de l'OEB a progressé de $6 \%$ par an en moyenne sur la période 1995-2005, tandis qu'à l'USPTO, les demandes ont progressé en moyenne de $7 \%$ par an (OCDE, 2007) ${ }^{9}$. 
Le paysage dans le secteur des brevets a de ce fait sensiblement évolué, avec l'apparition de nouveaux acteurs (universités) et le développement d'utilisations non traditionnelles des brevets (par exemple, cession de licences, mobilisation de capital). Il est important de garder ce contexte évolutif à l'esprit lors de l'interprétation des statistiques sur les brevets, s'agissant notamment des tendances dans le temps et des comparaisons entre pays ou entre branches.

\subsection{Informations contenues dans le document brevet}

Un document brevet contient une vaste somme d'informations, qui toutes présentent un potentiel pour l'analyse statistique. Cela est vrai non seulement de l'information bibliographique regroupée sur la page de garde, mais aussi du résumé, des revendications et de la description de l'invention, qui peuvent faire l'objet d'une analyse textuelle. Pour l'analyse statistique, l'information contenue dans un document brevet peut être subdivisée en trois catégories distinctes:

i) Description technique de l'invention.

ii) Développement et propriétés de l'invention.

iii) Historique de la demande.

La plupart des catégories d'informations expliquées ci-après sont disponibles quel que soit le bureau des brevets où la demande est déposée, car les informations requises et les procédures sont raisonnablement normalisées dans le monde. Une partie des informations sur les procédures ne sont pas disponibles en consultant le dossier de brevet lui-même, mais celles-ci sont néanmoins consignées et publiées par les bureaux des brevets, via d'autres voies.

\subsubsection{Description technique de l'invention}

- Titre et résumé (description de l'invention).

- Liste des « revendications ». Il s'agit de la description du contenu innovant; du champ d'exclusivité revendiqué. La revendication définit l'étendue de la protection assurée par les droits du brevet (limites juridiques). Celle-ci peut être plus ou moins large ou étroite, selon le contenu et le nombre des revendications.

- Les classes techniques dont relève l'invention (selon une classification des brevets). Celles-ci sont déterminées par les examinateurs. La classification la plus couramment utilisée est la classification internationale des brevets (CIB). Parallèlement, une classification des brevets nationale (par exemple USPC à l'USPTO) ou régionale (ECLA à l'OEB) figure dans le document brevet (ainsi, l'ECLA est très détaillée avec plus de 100000 catégories; c'est une ventilation de la CIB). 
- État antérieur de la technique. Chaque brevet indique l'état de la technique en rapport avec l'invention. Les références citées (brevets et autres) aident à définir les revendications du brevet et ses utilisations et applications spécifiques.

- Références du brevet. Il s'agit de citations de technologies antérieures applicables qui sont protégées par ou décrites dans d'autres brevets déposés où que ce soit dans le monde, à n'importe quelle date et dans n'importe quelle langue.

- Littérature hors brevets. Ce sont des publications scientifiques, comptesrendus de conférences, ouvrages, guides de base de données, manuels techniques, descriptions de normes, etc.

\subsubsection{Développement et propriété de l'invention}

- La liste des inventeurs et leurs adresses respectives. Les inventeurs sont des particuliers, en général des employés des demandeurs du brevet.

- La liste des demandeurs (assignees aux États-Unis) et leurs adresses respectives. Les demandeurs disposeront d'un titre légal sur le brevet (ils en seront propriétaires) si celui-ci est accordé. Dans la vaste majorité des cas, les demandeurs seront des entreprises, et les inventeurs leurs employés. Toutefois, il peut arriver qu'une même personne soit inventeur et demandeur (par exemple, inventeur indépendant) ${ }^{10}$.

\subsubsection{Historique de la demande}

- Numéro de publication, numéro de la demande, numéro du brevet (délivrance). Ces numéros se présentent de différentes façons, selon le bureau des brevets. Ils peuvent être utilisés comme identifiants pour la réalisation d'analyses des données dans les bases de brevets.

- Numéro de priorité. Il s'agit du numéro de demande ou de publication d'une demande prioritaire, le cas échéant. Elle permet d'identifier le pays de priorité, de reconstruire les familles de brevets, etc.

- Date de priorité. C'est la première date de dépôt d'une demande de brevet, où que ce soit dans le monde (en général auprès du bureau des brevets du pays du demandeur), en vue de protéger une invention. C'est la date la plus proche de celle de l'invention.

- Date de dépôt. C'est le premier jour à partir duquel la protection s'appliquera dans le pays concerné, si le brevet est accordé.

- Date de publication. Les brevets sont généralement publiés (c'est-à-dire que l'information est rendue publique) 18 mois après la date de priorité. Avant la publication du document brevet, le contenu du document demeure secret ${ }^{11}$. 
- Liste de désignation. Pour les demandes de brevets déposées en vertu des procédures de la Convention européenne sur les brevets ou du Traité de coopération en matière de brevets, les demandeurs sont tenus de désigner les pays membres dans lesquels la protection est demandée.

- Date de refus ou de retrait. Celle-ci indique que l'invention n'a pas rempli les critères statuaires (nouveauté, non-évidence ou applicabilité industrielle) de brevetabilité ou que le demandeur a décidé de suspendre la demande de brevet pendant le processus d'examen.

- Date de délivrance. Il existe un décalage entre la date de demande et la date d'approbation du brevet. De façon générale, il faut entre deux et huit ans pour qu'un brevet soit accordé.

- Date de déchéance. Un brevet peut être frappé de déchéance avant la date d'expiration statutaire si les droits de renouvellement ne sont pas acquittés ou s'il est révoqué par des tribunaux. Cette « information consécutive à la délivrance » est en général disponible auprès des "registres de brevets ", qui enregistrent également (selon le pays) les changements de propriété, les contrats de licence déclarés, etc. ${ }^{12}$.

\subsection{Les brevets comme indicateurs statistiques de l'activité d'invention}

Parmi les indicateurs disponibles sur la production de technologies nouvelles, ceux fondés sur les brevets sont sans doute les plus fréquemment utilisés. Les statistiques fondées sur les brevets ont plusieurs usages. Elles permettent de mesurer l'inventivité de pays, régions, entreprises ou inventeurs individuels, selon l'hypothèse que les brevets reflètent la production inventive et que plus les brevets sont nombreux, plus les inventions le sont également. Des recherches empiriques ont montré que les brevets sont fréquemment un bon élément de prévisions de la performance économique. Dans une étude auprès de 258 spécialistes de la R-D, Keller et Holland (1982) ont conclu que le nombre de brevets d'un inventeur est corrélé de façon significative avec des notations et une auto-notation de performances excellentes. Dans une étude de 1200 entreprises appartenant à des branches de haute technologie, Hagedoorn et Clood (2003) ont constaté que le nombre de brevets déposés par une entreprise donne une très bonne idée de sa performance technologique. Au niveau des pays, Rassenfosse et van Pottelsberghe (2008) ont mis en évidence une forte corrélation entre le nombre de brevets et la performance en matière de R-D.

Les statistiques des brevets servent également à retracer certains aspects de la dynamique du processus d'innovation (par exemple, coopération dans la recherche, diffusion de la technologie entre branches ou entre pays, etc.) ou du processus concurrentiel (stratégie commerciale des entreprises); elles sont également utilisées pour assurer un suivi du système de brevets lui-même. Les 
brevets sont par ailleurs utiles pour retracer les phénomènes de mondialisation. Ainsi, à partir de l'adresse des inventeurs, il est possible d'élaborer des indicateurs basés sur les brevets pour suivre l'internationalisation de la recherche, à savoir les activités de co-invention internationale en science et technologie ou la mobilité des inventeurs entre pays.

Bien que les demandes de brevets soient un indicateur de la recherche menée à terme dans un domaine particulier de recherche, les brevets ne rendent pas compte de la totalité des efforts de recherche ou d'innovation derrière une invention. D'autre part, une invention couverte par un brevet (nouveau produit ou procédé) n'a pas besoin d'être effectivement industrialisée. On sait que beaucoup de brevets ne sont pas du tout exploités, car après avoir soumis sa demande, l'inventeur réalise que la valeur économique de son invention n'est pas suffisante, ou une invention supérieure peut être mise plus rapidement sur le marché. Selon l'enquête PATVAL (2005), environ 40 \% des brevets de l'échantillon ne sont pas exploités à des fins industrielles ou commerciales pour des raisons stratégiques ou faute pour les détenteurs de disposer des actifs complémentaires en aval pour les exploiter : $18.7 \%$ ne sont pas utilisés et visent à bloquer les concurrents, et $17.4 \%$ sont considérés comme des « brevets dormants " qui ne sont pas du tout exploités.

Les brevets peuvent également être considérés comme une phase intermédiaire entre la R-D (en amont) et l'innovation (qui signifie que l'invention est exploitée en aval dans des processus économiques). Des brevets peuvent être obtenus à différentes phases du processus de R-D; c'est notamment le cas des inventions incrémentales ou cumulatives. En ce sens, les brevets peuvent être considérés non seulement comme un produit de la R-D, mais aussi comme un intrant dans l'innovation. À cet égard, ils peuvent être considérés les deux à la fois. Ce caractère intermédiaire fait que les données sur les brevets offrent une passerelle utile entre les données sur la R-D et les données sur l'innovation (recueillies les unes et les autres dans le cadre d'enquêtes auprès des entreprises).

Les données sur les brevets présentent des avantages et des inconvénients dans leurs capacités à rendre compte des activités d'invention. Leurs principaux avantages sont les suivants :

- Les brevets couvrent un large éventail de technologies sur lesquelles les autres sources de données sont parfois limitées (par exemple, nanotechnologies).

- Les brevets ont un lien étroit (sinon parfait) avec l'invention. Les inventions les plus importantes des entreprises sont brevetées, qu'elles soient ou non fondées sur la R-D.

- Chaque document brevet contient des informations détaillées sur le processus d'invention: une description raisonnablement complète de l'invention, le domaine technologique en question, les inventeurs (noms et adresses), les demandeurs (propriétaires), les citations renvoyant aux 
brevets antérieurs et les articles scientifiques en relation avec l'invention, etc. Le volume des données sur les brevets à la disposition des chercheurs est considérable. Plus d'un million de brevets sont demandés chaque année dans le monde et forment une source sans équivalent sur l'évolution de l'invention. Les données sur les brevets sont publiques, à la différence des données d'enquête qui sont généralement protégées par les lois sur le secret statistique.

- Le champ couvert par les données sur les brevets en termes géographiques et chronologiques est sans équivalent. On dispose de données sur les brevets pour l'ensemble des pays appartenant à un système de brevets, c'est-à-dire la quasi-totalité des pays du monde. Les données sur les brevets sont disponibles - parfois même sous forme électronique - depuis la naissance des systèmes de brevets, et elles remontent donc au XIX ${ }^{\mathrm{e}}$ siècle dans la plupart des pays de l'OCDE.

- Les données sur les brevets sont très facilement disponibles auprès des bureaux des brevets nationaux et régionaux. Le coût marginal pour les statisticiens est bien inférieur à celui de la réalisation d'enquêtes, même s'il reste parfois non négligeable (les données doivent être nettoyées, formatées, etc.). À la différence des données d'enquête, la collecte de statistiques sur les brevets n'impose aucune charge supplémentaire à l'unité déclarante (à savoir l'entreprise), car les données ont déjà été recueillies par les bureaux des brevets pour le traitement des demandes.

Toutefois, comme indicateurs de l'activité technologique, les brevets présentent aussi certains inconvénients :

- Les inventions ne sont pas toutes brevetées. Les inventions présentant peu de perspectives économiques peuvent ne pas justifier le coût du brevetage. Les inventions qui n'apportent qu'une contribution triviale à l'état de la technique ou les inventions non techniques ne remplissent pas les exigences de la protection par brevet. Des considérations stratégiques peuvent également conduire l'inventeur à préférer d'autres méthodes de protection (le secret). Par conséquent, les données sur les brevets peuvent ne pas rendre compte de ces inventions (voir par exemple, Pavitt, 1988).

- La propension à déposer des demandes de brevets diffère sensiblement selon les disciplines techniques. Ainsi, dans l'industrie électronique (par exemple, semi-conducteurs) un invention breveté peut être entouré par plusieurs brevets sur des inventions incrémentales de la première invention, l'objectif étant de dissuader l'entrée de nouveaux concurrents et de négocier des transactions de licences croisées avantageuses avec les concurrents. Cette stratégie "d'inondation de brevets" se traduit bien entendu par une multiplication du nombre des brevets dans certains domaines techniques. La propension à breveter diffère également selon les entreprises. Les entreprises 
nouvelles ou les petites et moyennes entreprises (PME) - notamment celles qui n'ont pas de production à grande échelle - ont davantage de difficultés à couvrir les coûts d'un brevet (bien que des politiques nationales s'attachent à résoudre ce problème en proposant aux PME des incitations ou des taxes réduites pour le dépôt de brevets).

- Plusieurs études ont montré que la répartition des brevets selon leur valeur est également faussée (par exemple Pakes and Schankerman, 1986; Harhoff et al., 1999). De nombreux brevets n'ont pas d'application industrielle (et n'ont donc que peu ou pas de valeur pour la collectivité), tandis qu'un nombre limité est de très grande valeur. Toutefois, la divulgation de l'information présente un bénéfice pour la société, car cela accroît le stock des connaissances. Avec une telle hétérogénéité, un simple comptage des brevets peut être trompeur. Cela n'est pas spécifique aux brevets car c'est une caractéristique majeure du processus d'invention qui s'applique également aux dépenses de R-D (dont une bonne partie ne débouche sur rien, alors que certaines activités limitées conduisent à des succès considérables).

- Les disparités dans les législations et pratiques en matière de brevets au niveau mondial limitent la comparabilité entre pays des statistiques des brevets. Il est donc préférable d'utiliser des données homogènes sur les brevets (provenant d'un même bureau des brevets ou d'un même ensemble de bureaux des brevets).

- Les changements dans les législations sur les brevets au cours de temps incitent à la prudence dans l'analyse des tendances temporelles. La protection accordée aux détenteurs à l'échelle mondiale a été renforcée depuis le début des années 80 et de ce fait, les entreprises ont une plus grande propension à breveter qu'auparavant. La liste des technologies couvertes s'est allongée au fil du temps et dans certains pays elle englobe maintenant le logiciel et les séquences génétiques qui étaient exclus auparavant. D'autres facteurs, telle la pratique des offices de brevets, peuvent avoir un impact appréciable sur les comptages de brevets, notamment sur le nombre de brevets délivrés sur une période donnée.

- Les données sur les brevets sont complexes, car elles découlent de processus juridiques et économiques eux-mêmes complexes. Il est donc important de prendre en compte tous ces facteurs lorsque l'on compile et interprète des données sur les brevets, faute de quoi on risque d'aboutir à des conclusions erronées.

La plupart des limitations indiquées ci-dessus peuvent être surmontées en utilisant des méthodologies adéquates à limiter l'impact des biais et des limitations des données. Ainsi, la dissymétrie dans la répartition des brevets en fonction de leur valeur peut être prise en compte en pondérant les 
comptages de brevets par le nombre de citations ou en sélectionnant un sousensemble des brevets de valeur similaire (par exemple, les brevets triadiques sont des brevets à forte valeur, voir le chapitre 4). De la même manière, pour surmonter les différences dans la propension à breveter selon les branches, il est possible de restreindre l'analyse à un secteur ou une branche, ou de pondérer les données en conséquence.

Selon la question posée, les données sur les brevets peuvent être utilisées conjointement avec d'autres données, comme les données sur la R-D ou les données des enquêtes sur l'innovation, pour étudier les performances en matière d'innovation et de technologie. Cette combinaison permet de corroborer ou d'infirmer les interprétations déduites séparément de chaque source de données et le rapprochement des données permet de tirer davantage d'informations (par exemple, dans certaines circonstances le degré de succès de la R-D peut être déduit des dépôts de brevets). Certains chercheurs ont rapproché les données sur les brevets à d'autres données, comme celles provenant d'enquêtes sur la R-D ou d'autres enquêtes auprès des entreprises (notamment des bases de données privées); d'autres ont mis au point des enquêtes spéciales pour compléter les données sur les brevets et ainsi mieux mesurer les variables qui les intéressent, par exemple enquête auprès des entreprises de technologie concernant leur utilisation des brevets (l'enquête Carnegie Mellon; Cohen et al., 2000) ou enquêtes auprès des inventeurs sur les processus ayant conduit aux brevets ou sur la valeur des brevets (Gambardella et al., 2005).

\subsection{Bases de données sur les brevets}

Les bases de données sur les brevets existent depuis longtemps. Ces bases, qui comprennent les informations bibliographiques (telles que décrites dans la section 2.3) et le texte intégral des brevets, sont des outils fondamentaux dans les procédures de recherche et d'examen réalisées dans les bureaux des brevets, car elles enregistrent l'état antérieur de la technique ayant fait l'objet de brevets. Au cours de la dernière décennie, ces bases ont été étendues et les données sur les brevets ont été couplées à d'autres informations : données sur les entreprises (par exemple, après normalisation des noms des demandeurs et appariement avec les listes de noms des entreprises), classification industrielle, codification des niveaux territoriaux (régions) en fonction des informations sur les adresses (inventeurs ou demandeurs, etc.).

Les bases de données sur les brevets peuvent aussi comprendre des informations additionnelles sur les procédures d'examen, telles que le statut juridique de l'examen, de même que sur le dépôt et la publication de la demande. Certains types de données sont plus rarement codifiés par les producteurs de données sur les brevets. Ainsi, les changements de propriété en cours de procédure d'examen ou sur la durée de vie d'un brevet sont rarement enregistrés 
dans les bases de données traditionnelles rendues disponibles par les bureaux des brevets.

Bien que les données sur les brevets soient produites par les autorités en charge des brevets, des bases de données sur les brevets exploitant ce type d'information sont également produites et publiées par des entités privées. Les utilisateurs doivent être attentifs à la nature des informations sur les brevets contenues dans les bases de données et aux types d'informations dont peuvent rendre compte les statistiques et indicateurs.

Certaines bases de données sur les brevets sont largement utilisées à des fins statistiques et de recherche : c'est notamment le cas des NBER Patent Citations Data Files créés par Jaffe, Trajtenberg et Hall avec l'aide de chercheurs du NBER et de l'Université Case Western Reserve University; de la base de données statistiques mondiales sur les brevets de l'OEB (également appelée EPO PATSTAT), créée par l'OEB avec le Groupe d'étude sur les statistiques de brevets de l'OCDE; et de la base de données sur les brevets de l'IIP (Institute of Intellectual Property), qui contient des données internes sur les brevets auprès du JPO (Seiri Hyojunka Data).

\subsection{Thèmes de recherche}

Les indicateurs et études fondés sur les données de brevets sont extrêmement divers quant au format de la publication (annuaire statistique, rapport d'analyse, travaux de recherche universitaires), au niveau d'agrégation des données compilées (nationales, régionales, entreprises, branches ou domaines techniques); à la démarche suivie (compilation d'indicateurs, réalisation d'estimations économétriques); et aux questions analytiques ou politiques prises en compte. On trouvera ci-après une liste non exhaustive de thèmes abordés dans l'abondante littérature exploitant les données sur les brevets.

- Performance technologique. Les brevets servent à suivre les performances technologiques des entreprises (ou d'autres organisations), des régions et des pays. Par rapport à d'autres indicateurs de production comme les publications, ces indicateurs sont mieux adaptés à des activités plus proches du développement technologique. Ils aident à suivre la domination ou le positionnement technologique dans un domaine ou une discipline technologique donné (par exemple, indicateurs des avantages technologiques révélés), et leurs évolutions dans le temps. En tant qu'indicateurs des performances technologiques, du niveau de spécialisation technologique et/ou de la force d'une région géographique ou d'un pays (ou d'une entreprise), ils aident les décideurs à identifier les points forts et les points faibles des systèmes d'innovation nationaux ou régionaux. 
- Technologies émergentes. Les indicateurs fondés sur les brevets sont un moyen unique - parfois sans aucun équivalent - de suivre l'apparition de technologies émergentes (par exemple, nanotechnologie, biotechnologie). Des domaines techniques particuliers peuvent être reconstruits au moyen de mots-clés ou de recherche dans les résumés et description des brevets. L'information détaillée figurant dans le document brevet permet d'identifier les entreprises ou agences actives dans ces domaines, les modes d'invention (par exemple, collaboration inter-institutions), la géographie des pôles technologiques, etc. Les données sur les brevets peuvent être utilisées conjointement avec celles des publications scientifiques. Les enquêtes auprès des entreprises viennent en général à un stade plus tardif, car elles nécessitent une connaissance préalable précise du domaine (notamment des entités actives).

- Diffusion des connaissances et dynamique du changement technologique. Parce qu'ils fournissent une description détaillée de la façon dont les inventions ont été réalisées et de l'état antérieur de la technique, les brevets sont un bon indicateur du transfert de connaissances. Les citations de brevets permettent de suivre l'influence des inventions antérieures dans des inventions nouvelles. Il est donc possible d'identifier l'influence d'inventions ou d'ensembles d'inventions spécifiques, et de suivre leur diffusion au sein de l'économie. Les citations d'autres brevets ou de littérature hors brevets (notamment les publications scientifiques) sont utiles pour quantifier les transferts de connaissances entre organisations (par exemple d'entreprise à entreprise ou d'université à industrie), régions géographiques et/ou domaines technologiques, ainsi que les retombées de connaissances issues d'entités innovantes spécifiques (par exemple des multinationales vers des entreprises nationales, ou des centres publics de recherche vers l'industrie).

- Géographie de l'invention. Les adresses de l'inventeur et du demandeur étant communiquées, les brevets peuvent être répartis entre régions avec beaucoup de détail (bien que cela implique un volume de travail non négligeable, dans la mesure où les données brutes ne sont pas toujours bien formatées). Les données sur les brevets peuvent donc être exploitées pour étudier les propriétés géographiques des processus d'invention, le rôle des acteurs locaux dans l'innovation régionale ou nationale (universités, petites entreprises, grandes entreprises, etc.), leurs interactions et le profil et l'impact de la spécialisation technologique régionale, etc. ${ }^{13}$.

- Créativité et réseaux sociaux. Les informations issues des documents brevets peuvent être utilisées pour retracer les évolutions de carrière et les performances des différents inventeurs (par exemple, leur domaine d'activité, leur localisation, leur employeur) ou pour analyser les réseaux d'inventeurs (qui invente avec qui, etc.). 
- Valeur économique des inventions. La valeur technique d'une invention est une indication importante de son impact économique. Les données sur les brevets donnent un accès privilégié à l'information concernant la valeur technique des inventions. Des corrélations ont été démontrées entre la valeur économique d'un brevet et le nombre de ses citations (dans des brevets ultérieurs); cette information peut être exploitée pour compiler des indicateurs de la valeur relative des brevets. En mettant en correspondance les noms des demandeurs avec des données sur les entreprises, les données sur les brevets peuvent être reliées à des données économiques comme les données boursières, les données comptables, etc.

- Performance et mobilité des chercheurs. Le nom de l'inventeur figurant dans le document brevet, il est possible d'étudier certains aspects de l'activité d'invention au niveau du chercheur individuel. Cela implique un gros effort de nettoyage des données, dans la mesure où l'identification d'individus dans des bases de données comptant des millions de noms n'est pas une tâche simple. Cette information peut être utilisée pour étudier des questions comme la mobilité des chercheurs (entre entreprises ou entre pays), établir des profils des différences entre domaines, savoir qui travaille avec qui, étudier la problématique homme-femme (quand cela est possible avec l'aide de données complémentaires), etc. (Trajtenberg et al., 2006).

- Rôle des universités dans le développement technologique. L'impact des universités dans le développement technologique peut être observé à travers les brevets que celles-ci ont déposés et en compilant le comptage de ces brevets ainsi que leurs citations (dans des brevets ultérieurs), etc. Il peut également être observé à travers les citations dont bénéficie la recherche universitaire dans les brevets déposés par l'industrie (Narin et al., 1999). Dans un nombre croissant de pays, les comptages de brevets servent aux organismes de financement et aux ministères pour évaluer les performances des institutions universitaires et des différents chercheurs.

- Mondialisation des activités de R-D. Les brevets contiennent des informations sur les performances et les activités d'invention des entreprises. À travers les adresses des demandeurs et des inventeurs, il est possible de suivre les évolutions et l'intensité de la co-invention internationale (mesure de la collaboration de recherche entre inventeurs implantés dans des pays différents), la propriété étrangère des inventions nationales, et vice versa.

- Stratégies des entreprises en matière de brevets. L'historique de la demande de brevet est également disponible dans le document brevet. Il donne la chronologie de l'invention, le cheminement de la demande au sein du bureau des brevets et les stratégies du demandeur (états désignés, équivalents du brevet et dates de priorité, etc.). Cette information est utile pour identifier la 
stratégie commerciale du détenteur du brevet, notamment les pays pour lesquels la protection est demandée, et leur ordre d'importance.

- Évaluation de l'efficacité du système de brevets. Les données sur les brevets peuvent aussi être utilisées pour évaluer les effets du système de brevets sur les inventions et leur diffusion. Dans quelle mesure et par quelles voies l'économie bénéficie-t-elle du système de brevets? Dans quelle mesure certaines stratégies ayant prétendument des impacts négatifs sur la collectivité (blocage, cloisonnement, etc.) sont-elles adoptées par le demandeur? Quels sont les effets de politiques spécifiques en matière de brevets sur la performance économique nationale?

- Prévision des demandes de brevets. Les séries chronologiques compilées sur les brevets sont également utiles pour prévoir la demande future de brevets, et facilitent ainsi la planification budgétaire des bureaux des brevets.

- Suivi du fonctionnement interne du système de brevets. Les données sur les brevets peuvent aussi être exploitées pour suivre le système de brevets lui-même, c'est-à-dire le volume de l'activité de brevetage par les entreprises, la façon dont les bureaux des brevets fonctionnent, etc. Toutefois, cette exploitation des données sur les brevets n'est pas l'un des axes principaux de ce manuel, qui porte sur les données des brevets comme indicateur de technologie. Très souvent, le suivi du système de brevets exigera l'utilisation de règles statistiques différentes. Ainsi, des dates purement administratives (par exemple, date de délivrance du rapport de recherche), qui présentent peu d'intérêt d'un point de vue économique, peuvent être extrêmement importantes pour évaluer la performance interne d'un bureau des brevets. Ce type d'exploitation des données sur les brevets est principalement pratiqué par les bureaux des brevets eux-mêmes (voir le rapport statistique trilatéral annuel publié conjointement par l'OEB, le JPO et l'USPTO, ou les diverses publications statistiques de l'OMPI).

\section{Notes}

1. Certaines juridictions assurent une période de protection plus longue pour certaines inventions (par exemple, médicaments) de manière à compenser les délais administratifs dans la délivrance de l'approbation de mise sur le marché.

2. Bien que la plupart des méthodologies et indicateurs de brevets décrits ici s'appliquent aux brevets (connus sous le nom de « utility patents " aux États-Unis) et modèles d'utilité (ou brevets d'utilité), nous mettrons l'accent sur les premiers car les brevets sont un droit de propriété intellectuelle davantage standardisé en ce qui concerne les inventions à l'échelle mondiale que l'autre forme de protection. Les modèles d'utilité ou " petits brevets ", à l'instar des brevets, donnent à leurs détenteurs une exclusivité sur le marché. Par rapport aux brevets, la protection est moindre (durée plus courte, souvent de 6 à 10 ans) et elle est plus facile à obtenir (exigences moins contraignantes pour la brevetabilité). Les modèles d'utilité n'existent pas dans tous les pays. 
3. Cette procédure permet de revendiquer la priorité tout en sauvegardant le droit de déposer effectivement par la suite des demandes de brevets dans des pays membres ultérieurement. Une demande de brevet internationale comprend deux phases : la première est la phase internationale dans laquelle la protection par brevet est pendante dans l'attente d'une première demande de brevet auprès d'un bureau des brevets d'un État partie au PCT. La deuxième phase est la phase nationale et régionale, qui suit la phase internationale, dans laquelle les droits sont maintenus en déposant les documents nécessaires auprès des bureaux des brevets des différents États contractants parties au PCT. La décision d'accorder un brevet reste la responsabilité de chacun des bureaux nationaux ou régionaux désignés.

4. Toutefois, en Europe, la procédure centralisée d'opposition à un brevet européen et la procédure d'appel centralisée à l'égard d'un brevet européen sont une alternative à l'action en justice pouvant conduire à la révocation d'un brevet européen.

5. Selon les règles de l'OMPI, les codes INID à deux lettres (identification numérique internationale des données) sont utilisés pour identifier les éléments bibliographiques sur la page de garde du document brevet. Ils contribuent à harmoniser l'usage et la présentation des spécifications et de la documentation connexe des brevets, et permettent de diffuser l'invention sans avoir recours à des traductions ou des scripts.

6. Toutefois, les secrets commerciaux font l'objet d'une protection juridique dans le cadre des ADPIC (voir l'article 39).

7. L'importance et la durée du pouvoir de marché dépendent de plusieurs facteurs, par exemple le degré de substitution entre technologies, le rythme du changement technologique, etc.

8. Certaines pratiques dans l'exploitation des brevets peuvent restreindre la concurrence sur les marchés de technologie d'une manière qui va au-delà des droits conférés par la législation sur la propriété intellectuelle, par exemple le fait de lier à des inventions brevetées la vente d'autres produits ou matériaux non brevetés (ventes groupées), de restreindre les activités commerciales du titulaire de la licence en dehors du champ couvert par le brevet, d'imposer un pouvoir de veto sur l'octroi de nouvelles licences, d'imposer des commissions qui ne sont pas raisonnablement liées à la vente de produits brevetés, etc.

9. En revanche, le nombre des demandes de brevets auprès du JPO est demeuré relativement stable sur la période 1991-2005 (OCDE, 2007).

10. Les changements de propriété au fil du temps ne sont pas toujours enregistrés dans les bases de données des brevets. Dans la majorité des bureaux des brevets, la dernière information communiquée indique le(s) dernier(s) propriétaire(s) enregistré(s), et l'enregistrement d'un nouveau propriétaire, dans l'éventualité d'un tel changement, n'est pas obligatoire.

11. Dans certains cas, les demandeurs peuvent demander la publication anticipée de la demande de brevet, avant les dates habituelles (voir chapitre 3, section 3.3.2).

12. Dans certains bureaux, les demandes de brevets peuvent être également retirées, pendant leur examen, en raison du refus ou du non-paiement des redevances, du fait d'un " retrait induit » à la suite d'un rapport de recherche défavorable, ou pour des raisons commerciales propres au demandeur.

13. Il faut être vigilant dans l'interprétation des données géographiques sur les brevets, notamment en ce qui concerne les activités des entreprises, dans la mesure où leurs activités de recherche sont géographiquement dispersées et où l'adresse de l'invention n'est pas nécessairement la même que celle où la recherche a été effectuée. 


\section{Références}

Blind, K., J. Edler, R. Frietsch et U. Schmoch (2006), « Motives to Patent: Empirical Evidence from Germany ", Research Policy, vol. 35, pp.655-672.

Cohen, W.M., R.R. Nelson et J.P. Walsh (2002), « Links and Impacts: The Influence of Public Research on Industrial R\&D ", Management Science, 48, $n^{\circ} 1$, January, pp.1-23.

Cohen, W.M., R.R. Nelson, et J.P. Walsh (2000), « Protecting Their Intellectual Assets: Apropriability Conditions and Why US Manufacturing Firms Patent (or Not) ", NBER Working Paper $\mathrm{n}^{\circ}$ W7552.

Gambardella, A., D. Harhoff et B. Verspagen (2005), « The Value of Patents ", mimeo.

Goto, A. et A. Nagata (1997), « Technological Opportunities and Appropriating Returns from Innovation: Comparison of Survey Results from Japan and the US ", NISTEP Report $\mathrm{n}^{\circ} 48$, National Institute of Science and Technology Policy, March.

Guellec, D. et B. van Pottelsberghe (2007), The Economics of the European Patent System, Oxford University Press.

Hagedoorn, J. et M. Cloodt (2003), « Measuring Innovative Performance. Is there an advantage in using multiple indicators? ", Research Policy, 32, pp.1365-1379.

Keller, R.T. et W.E. Holland (1982), « The Measurement of Performance among R\&D Professional Employees: A Longitudinal Analysis ", IEEE Transactions of Engineering Management, 29: pp.54-58.

Levin, R.C., A.K. Klevorick, R.R. Nelson et S.G. Winter (1987), « Appropriating the Returns from Industrial Research and Development ", Brookings Papers on Economic Activity, vol. 3, pp.783-820.

Pavitt, K. (1988), "Uses and Abuses of Patent Statistics ", in A.F.J. van Raan (éd.), Handbook of Quantitative Studies of Science and Technology, Elsevier Science Publishers, Amsterdam.

de Rassenfosse, G. et B. van Pottelsberghe (2008), « A Policy Insight into the R\&D Patent Relationship ", ULB Working Paper.

Scotchmer, S. (2004), Innovation and Incentives, MIT Press.

Trajtenberg M., G. Shiff et R. Melamed (2006), « The “Names Game”: Harnessing Inventors' Patent Data for Economic Research », NBER Working Papers 12479, National Bureau of Economic Research, Inc. 


\section{ANNEXE 2.A1}

Graphique 2.A1.1. Page de garde d'une demande de brevet à l'OEB

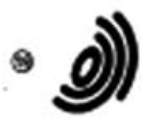

Europlisches Patentamt

Europoen Patont Offlco

Office europben des brevets

28. Puetcosion number:

0046310

A1

(4)

\section{EUROPEAN PATENT APPLICATION}

(11) Aspleationnumber: E1tossp.1

(2) Dun of tikng: 18.10.7s

(9) m. as: H 02 K 15/02, H 01 F 29/10, H01F 41/02

Q Pricrity: 181078 aw645errs

(4) Dute of puttcasion of applisation: 24.02:2 Dutotis cars 8) Doslosated Coctracting Sranes: Ar BE CH DE FR GB IT
LU NE $8 E$

9. Pelcatisn numteer of the earlier appleatisn in actordinte whth Art. 78 Eoc: 0010 sss
(20) Acpisark: Cass-0-Metie Pty. Ut, 20 MaEtvey Strees, Woblabo Nee Bash Wales, 2017 (AU)

6. Inves: Btariey, Louls, 22 Peryare Asos, Beverley HIts New South Waise 2009 (AU)

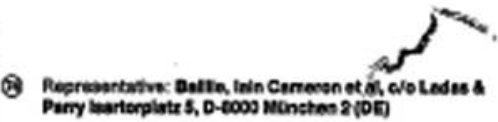

\section{Eectical oqupment and its tatricason.}

(2) An indective doctric mastina auch as in havetien moter or a transiormer hrutro thald and robor cores, or. resposttrely. primary and saccondary cores, oach sach core beito fommed of metal strib [200] punchad to heve a slirality of hales spoved and located at prodestemined positbors along the strlp (204) 80 thot, when the otrip is wound abses a central exs, the toles (2t6, 2t6. 220) are becised so is is form raclally eocenting zlots lans.

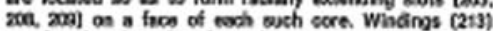
con be sleced in the slots of the field ecre and is the islots of tratslomer corea. A cordactor [2003] can be 'sleced in the shate of the retor cora.

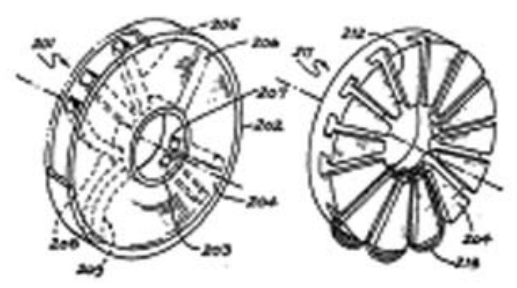




\section{Graphique 2.A1.2. Exemple de page de garde d'une demande de brevet au JPO}

Il s'agit d'un exemple et non de la reproduction d'une demande effective

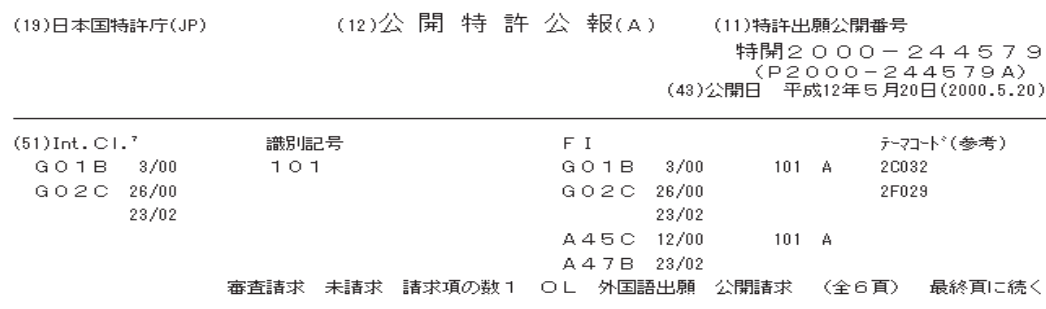

\begin{tabular}{|c|c|}
\hline (21)出䫝番号 & 特願平11-123456 \\
\hline （22）出願曰 & 平成11年11, 月10日(1999.11.10) \\
\hline (31)優先梏主張番号 & 83304359.9 \\
\hline (32)畷先日 & 平成10年11月12日(1989.11.12) \\
\hline (33)㮩先矢主張国 & フラン (FR) \\
\hline
\end{tabular}

特許洼第30条第 7 愐適用甲請有り 平成10年9月21日讨 画像工学会研究専門秀貝全主摧W1992年庶画像符号化

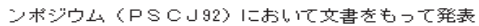

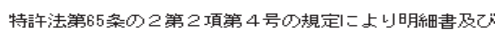
图面の一部阶捍㦲さする。

\begin{tabular}{|c|c|}
\hline (71)出願人 & 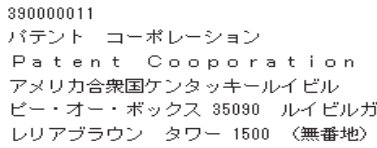 \\
\hline (71)出靨人 & 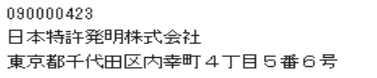 \\
\hline (72)発明者 & $\begin{array}{l}\text { 無明 大郎 } \\
\text { 神萗川県撗須脅市壱 1丁目2200番地 }\end{array}$ \\
\hline (74)代理人 & $\begin{array}{l}123456789 \\
\text { 并理士 代理 太息 }\end{array}$ \\
\hline
\end{tabular}

(54)【然明の名称]コッりシミり走查装置

(57) [要約] (传正有)

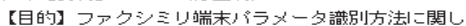

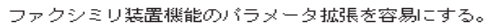

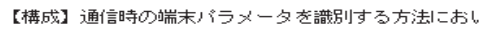

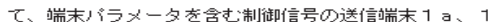

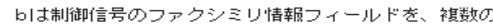
サア゙コィールドに分離し、客サア゙コィールドの情報を分

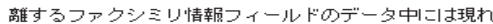

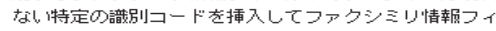

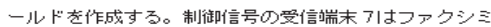

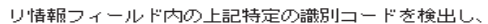

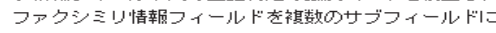

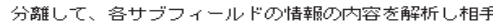
端末 $\omega$ 㟨末 ラメータを摭張する場合はユニーっコードを挿入して可

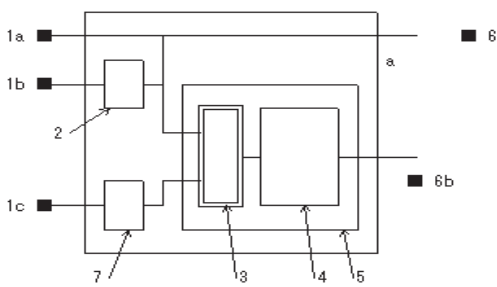
变骨の㟨末小ラ 
Graphique 2.A1.3. Page de garde d'une demande de brevet de l'USPTO

(9) United States

US $20000045039 \mathrm{Al}$

(12) Patent Application Publication Conti et al.

(10) Pub. No.: US 2008/0045039 A1

(43) Pub. Date: Feb. 21, 2008

(44) MrTHod of FORMING NIRIDE Fu.Ms WTH HIGH COMPRFSSIVE STRESS YOR MPRONED PFET DEVTE PERFORMAYC

(15) Imetices Rkbard A. Ceati, Kisolah NY (US): Reandd P. Bearese, Wropiogers Falls NY (US); Naber R. Klombo, Hopeces! Jantica. NY (tSk Anita Madan. Dusbary, NY (US), Miklasl C. Seis.s. Roullatpic, NY (US), Ray II. Thèman, Siensille, NY ASX Kwoeg Ben Wane. Wirgingers Folk NY (US): Daruen Yanz Horeard Joction. NY (US)

Correspoudries Aalres:

INTEXXATtONAL. BUSNESS M.MCIINXS

CORPORATION

DE.PT. 186

BLDG. 100-88:

2070 ROUTE 52

HOPEWEL. JUCTION NY 1253 (US)

(7) Asigass INTERx.Itova. Bustvxs MACHIVS CORFORsTION Anmik. NY (US) NONE.tUS SYS TEMS, IVC. Son be, CA (US)

(21) Appl. No: 11s73721

(22) Filat: Oet. 17, aw?

Related US. Applikatha Data

(62) Diviain of applicative $\mathrm{Na}$. I1/lea, vos, fild co Ju. 6. $2 \times 16$

Putlicabion Clessifcutho

(51) In. $\mathrm{C}$.

Holl 2131 (200601)

(52) U.X Cl. ...

(57)

ABSTRACT

A rectiod is provibed for meliog a FET devioe in which a airible liger oneties the PFIT ges strovure, where te ahise layer las a coctpressive stress with a mapinate

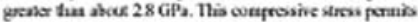
inporvol devise perfinuase in the PFET. The sitnile lyet

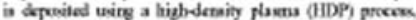
ubrvin to subetnte is ásocoud co an dectrode to utich : bis power in the raspe of abous $50 \mathrm{~W}$ to $3 \mathrm{cot}^{500} \mathrm{~W}$ is spptiod. The biss poucr is cterocterized as thigh-frequexy

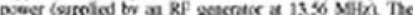

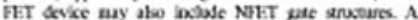
bbaing layer is deposted oner the NFHT pate strocares 20

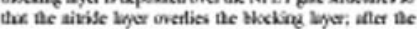
bbatirg lyor is renowod, the niride laye is act is ovetact with the NFET gove vrotuns. The titnde toger bas a thickess in the neye of ahoer $300-2000 \mathrm{~A}$

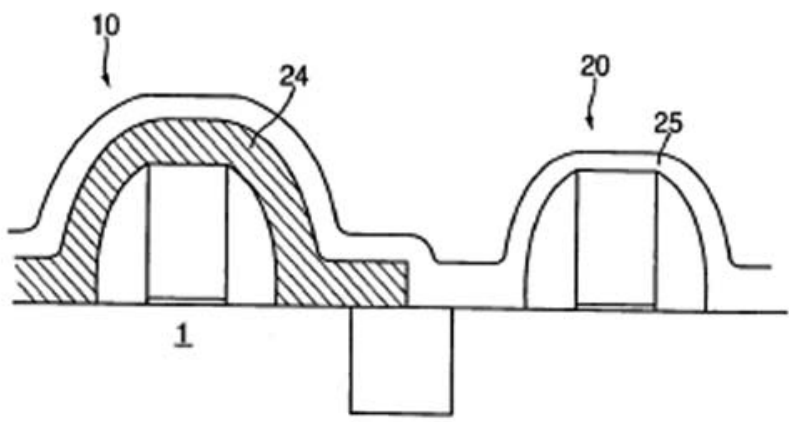




\section{Graphique 2.A1.4. Page de garde d'une demande PCT}

\section{(12) INTERNATIONAL APPLICATION PUBLISHED UNDER THE PATENT COOPERATION TREATY (PCT)}

(19) World Intellectual Property Organization International Bureau

(43) International Publication Date 18 December 2003 (18.12.2003)

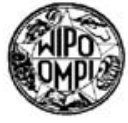

PCT

\section{||||||||||||||||||||||||||||||||||||||||||||||||||||||||||||||||||}

(10) International Publication Number WO 03/104470 A2
(51) International Patent Classification?: $9 / 12,15 / 11,15 / 79, \mathrm{~A} 61 \mathrm{~K} 48 / 00$

(21) International Application Number: PCT/CA03/00850

(22) International Filing Date:

5 June 2003 (05.06.2003)

(25) Filing Language:

English

(26) Publication Language:

English

(30) Priority Data:

$60 / 386,640$

5 June 2002 (05.06.2002) US

(71) Applicant (for all designated States except US): HER MAJESTY IN RIGHT OF CANADA As represented By the MINISTER OF AGRICULTURE AND AGRI-FOOD CANADA [CA/CA]; Agriculture and Agri-food Canada, Saskatchewan Research Centre, 107 Science Place, Saskatoon, Saskatchewan S7N 0X2 (CA).

(72) Inventors; and

(75) Inventors/Applicants (for US only): ROZWADOWSKI,
Kevin, L. [CA/CA]: 86 Harvard Crescent, Saskatoon, Saskatchewan S7N 3RI (CA). LYDIATE, Derek, J. $[\mathrm{GB} / \mathrm{CA}] ; 101$ Albert Street, Saskatoon, Saskatchewan S7N 1 E6 (CA).

(74) Agents: Kingwell, Brian, G. et al.; Smart and Biggar Box 11560 , Vancouve 650 West Georgia Street, Suite 2200 Vancouver, British Columbia V6B 4N8 (CA).

(81) Designated States (national): AE, AG, AL, AM, AT (utility model), AT, AU, AZ, BA, BB, BG, BR, BY, BZ, CA, $\mathrm{CH}, \mathrm{CN}, \mathrm{CO}, \mathrm{CR}, \mathrm{CU}, \mathrm{CZ}$ (utility model), CZ, DE (util ity model), DE, DK (utility model), DK, DM, DZ, EC, EH (utility model), EE, ES, FI (utility model), FI, GB, GD, GE, GH, GM, HR, HU, ID, IL, IN, IS, JP, KE, KG, KP, KR, KZ, LC, LK, LR, LS, LT, LU, LV, MA, MD, MG, MK, MN, MW, MX, MZ, NI, NO, NZ, OM, PH, PL, PT, RO, RU, SC, SD, SE, SG, SK (utility model), SK, SL, TJ, TM, TN, TR, TT, TZ, UA, UG, US, UZ, VC, VN, YU, ZA, ZM, ZW.

(84) Designated States (regional): ARIPO patent (GH, GM KE, LS, MW, MZ, SD, SL, SZ, TZ, UG, ZM, ZW) Eurasian patent (AM, AZ, BY, KG, KZ, MD, RU, TJ, TM), European patent (AT, BE, BG, CH, CY, CZ, DE, DK, EE,

[Continued on next page]

(54) Title: RETRONS FOR GENE TARGETING

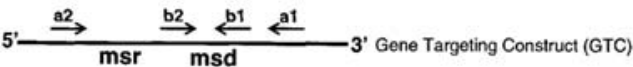

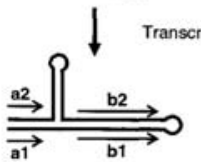

Gene Targeting Message RNA (gtmANA)

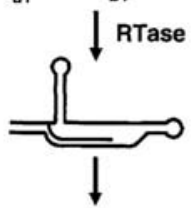

Self-priming

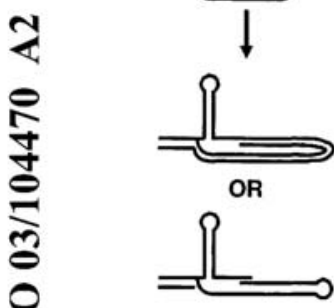

(57) Abstract: The invention provides methods and nucleic acid constructs that may be used to modify a nucleic acid of interest at a target locus within the genome of a host. In some aspects, the invention contemplates producing in vivo a gene targeting substrate (GTS), which may be comprised of both DNA and RNA components. The gene targeting substrate may comprise a gene targetig nucleotide sequence (GTNS), which is homologous to the target locus, but comprises a sequence modification compared to the target locus. The gene targeting substrate may be produced by reverse transcription of a gene targeting message RNA (gtmRNA). The gene targeting message RNA may be folded for self-priming for reverse transcription by a reverse transcriptase. The gene targeting message RNA may in turn be the product of transcription of a gene targeting construct (GTC) encoding the gene targeting message RNA. The gene targeting construct may for example be a DNA sequence integrated into the genome of the host, or integrated into an extrachromosomal element. Following expression of the gene targeting systems of the invention, hosts may for example be selected having genomic modifications at a target locus that correspond to the sequence modification present on the gene targeting nucleotide sequence.

In some embodiments, the structure of retrons may be adapted for use in the gene targeting systems of the invention. 

Chapitre 3

Systèmes et procédures en matière de brevets 


\subsection{Introduction}

Pour faire breveter une invention, la personne ou l'institution (entreprise ou institution publique ou privée, par exemple université ou organisme gouvernemental) propriétaire de l'invention doit déposer une demande auprès d'un bureau des brevets. Le demandeur qui souhaite faire protéger son invention dans plusieurs pays peut déposer une demande de brevet dans chaque pays séparément, déposer une demande de brevet auprès d'un bureau régional ou déposer une demande de brevet au Bureau international des brevets et demander l'entrée dans la phase nationale dans chaque pays pour lequel il sollicite une protection par brevet.

La demande et le traitement des brevets suivent des règles et procédures administratives et juridiques strictes, fixées dans des traités internationaux et des textes nationaux (lois et réglementations). Ces procédures et règles ont un impact direct sur la valeur et la signification des données sur les brevets. Il importe de les prendre en compte dans l'interprétation des statistiques sur les brevets, d'autant que ces règles ne sont pas totalement harmonisées entre les pays et ont évolué au fil du temps, et que des variations mineures dans la procédure peuvent avoir un effet spectaculaire sur les chiffres ultimes.

On trouvera dans ce chapitre une synthèse des procédures de dépôts de brevets dans les principaux bureaux des brevets et pour les principales voies: l'Office européen de brevets (OEB), le Japan Patent Office (JPO), le Patent and Trademark Office des États-Unis (USPTO) et le Traité de coopération en matière des brevets (PCT). Après une présentation des règles communes à tous les bureaux des brevets, on trouvera une analyse des variantes nationales et régionales, puis un examen des procédures applicables aux demandes internationales.

La procédure de délivrance des brevets, les exigences imposées aux demandeurs et parfois l'étendue des droits exclusifs varient largement selon les pays, en fonction des lois nationales et des accords internationaux. Comme on le verra, toutes les demandes de brevets, qu'elles soient internationales ou régionales, devront en définitive bénéficier d'un statut national, car elles doivent être validées par les bureaux des brevets nationaux. En conséquence, les spécificités nationales, en ce qui concerne aussi bien la procédure d'établissement du brevet que les activités suivant sa délivrance (par exemple, procédure de maintien des droits, de mise en application et d'invalidation) déterminent la façon dont les brevets fonctionnent dans la vie économique. Ces aspects doivent être pris en compte lors du choix de données 
spécifiques sur les brevets, et du calcul et de l'interprétation des indicateurs sur les brevets.

\subsection{Procédure de base}

La procédure d'obtention d'un brevet comprend plusieurs étapes qui sont similaires dans tous les pays :

- Premièrement, l'entité souhaitant bénéficier d'une protection par brevet (généralement une entreprise, mais ce peut être une personne, une université ou un organisme gouvernemental) doit déposer une demande de brevet auprès d'un bureau des brevets. Dans la demande, le demandeur doit décrire l'invention avec suffisamment de détails pour permettre à une personne du métier de compétence moyenne de la comprendre et de la mettre en œuvre. La partie la plus importante de la demande est la section sur les revendications, qui est une liste d'aspects de l'invention pour lesquels le demandeur revendique des droits exclusifs. Le demandeur doit acquitter certaines taxes administratives, qui varient considérablement d'un bureau des brevets à l'autre ${ }^{1}$.

- Deuxièmement, le bureau des brevets désigne un examinateur (ou un groupe d'examinateurs, avec un responsable) qui prend en charge la demande et qui est supposé être un expert dans le domaine technique en question. En général, l'examinateur effectue d'abord une recherche de nouveauté, qui consiste à vérifier les documents sur l'état antérieur de la technique jugés pertinents pour l'invention considérée. Ces documents décrivent les précédents dans les publications scientifiques et techniques en rapport avec l'invention (ou une partie de celle-ci) et ils constituent l'état antérieur de la technique auquel la nouveauté de l'invention sera comparée. De façon générale, seuls les documents qui ont été publiés avant la date de dépôt de la demande (ou le jour de dépôt de la demande de priorité, selon le cas) doivent être pris en compte dans la recherche. Le document de demande de brevet, auquel est joint le rapport de recherche, sont rendus publics 18 mois après la date de dépôt (sauf pour certaines demandes auprès de l'USPTO) ${ }^{2}$.

- Troisièmement, l'examinateur (généralement mais pas nécessairement le même que dans la deuxième phase) étudie la demande de brevet pour décider si l'invention est "non évidente » et implique une " activité d'invention " par rapport à l'état antérieur de la technique mis en évidence par la recherche effectuée. Le demandeur a le droit de soumettre une opinion écrite (pour débattre des conclusions de l'examinateur et de l'interprétation des publications trouvées) et de modifier si nécessaire le champ des revendications définies dans la demande. La délivrance signifie qu'aucune raison de refus n'a été trouvée dans la mesure où tous les critères de brevetabilité sont couverts : domaine brevetable, nouveauté, inventivité (nonévidence pour une personne du métier) et applicabilité industrielle (voir encadré 3.1). 


\section{Encadré 3.1. Critères de brevetabilité}

- Domaine : pour être brevetable, une invention doit relever de certains domaines de connaissance, que l'on peut qualifier en gros de technologiques. La loi est plus spécifique et varie dans une certaine mesure selon les juridictions. Les créations esthétiques, les lois de la nature et les idées abstraites sont exclues dans toutes les juridictions. Le logiciel est brevetable aux États-Unis, de même que les procédés commerciaux. La pratique dans ces deux domaines est plus restrictive au Japon, et plus encore en Europe (qui exclut le « logiciel en tant que tel »).

- Nouveauté : pour être brevetable, une invention doit avoir un caractère d'absolue nouveauté. Cela signifie qu'elle n'était pas à la disposition du public sous quelque forme que ce soit avant la date de demande du brevet, et qu'elle n'était décrite dans aucune publication avant cette date. La nouveauté est un concept mondial; une invention n'est pas jugée nouvelle dans un pays s'il est constaté qu'elle fait partie de l'état antérieur de la technique dans un autre pays, dans quelque langue que ce soit, à n'importe quelle période.

- Non-évidence/activité inventive : même quand il apparaît qu'une invention présente un caractère de nouveauté au sens strict, celle-ci peut néanmoins ne pas être brevetable si elle est considérée comme évidente pour une personne de métier maîtrisant raisonnablement son art. Le terme " évidente » a un sens juridique et il est donc utilisé dans des sens fort différents d'un pays à l'autre. Les critères d'invention et de nonévidence reflètent les mêmes exigences générales de brevetabilité figurant dans la plupart des législations sur les brevets, selon lesquelles une invention doit être suffisamment inventive, c'est-à-dire ne pas être évidente, pour être brevetée. L'expression " activité inventive " est surtout utilisée par exemple en Allemagne, au Royaume-Uni et dans les pays parties à la Convention sur le brevet européen (CBE), tandis que l'expression " non-évidence » est surtout utilisée dans la législation sur les brevets des États-Unis. Aux États-Unis, une chose est considérée comme évidente si les différences entre l'objet de la demande de brevet et l'état antérieur de la technique sont telles que l'objet dans son ensemble serait, au moment où l'invention a été effectuée, évident pour une personne ayant des compétences moyennes dans la technique dont relève l'objet. En Europe, la demande de brevet implique une activité inventive si elle résout un problème technique de façon non évidente.

Applicabilité industrielle : cette exigence vise principalement à distinguer entre les inventions esthétiques et scientifiques. Le terme " industrie " est interprété au sens large; ainsi il englobe l'agriculture. Mais il exclut les méthodes de traitement du corps humain ou animal par chirurgie ou thérapie et la pratique de méthodes de diagnostic sur le corps humain ou animal. Les machines dites à mouvement perpétuel ne répondent pas non plus à ces critères. Aux États-Unis, cette exigence est qualifiée d'utilité; toutefois, l'interprétation et le champ couvert par ce terme sont généralement les mêmes que ceux de l'application industrielle. Les traités internationaux sur les brevets utilisent souvent “ utilité » et " applicabilité industrielle » de façon interchangeable. 
- Quatrièmement, une fois accordé, le brevet peut être maintenu pour une durée maximale de 20 ans à compter de la date de dépôt ${ }^{3}$. Le détenteur du brevet est tenu de payer des frais de renouvellement (annuels dans la plupart des pays) au bureau des brevets pour maintenir en vigueur le brevet. Le bureau des brevets révoquera les brevets qui ne sont pas renouvelés. Un brevet peut être contesté, généralement par des concurrents qui considèrent que le brevet n'est pas valide et qu'il ne devrait pas avoir été accordé, le bureau des brevets n'ayant pas pris en compte une carence importante dans la demande de brevet ou n'ayant pas correctement appliqué les textes. Un brevet peut être contesté au sein du bureau des brevets lui-même dans certaines juridictions (par exemple, opposition à l'OEB; réexamen a l'USPTO a travers les conseils d'appel; système expérimental de procédure d'invalidation au JPO), de même que devant les tribunaux. Les tribunaux sont l'instance ultime dans l'application de la législation sur les brevets.

Les brevets déposés auprès d'un bureau national (ou régional) n'assurent une protection qu'au sein de leur juridiction. Ainsi, un brevet délivré par l'USPTO n'assurera une protection qu'à l'intérieur des États-Unis. Si l'inventeur (demandeur) souhaite protéger la même invention au Japon, il devra déposer une demande de brevet distincte auprès du JPO, soit directement, soit via le Traité de coopération en matière de brevets (PCT) à l'Organisation mondiale de la propriété intellectuelle (OMPI). Le dépôt auprès de l'OMPI n'empêche pas le demandeur de déposer des demandes auprès des bureaux nationaux.

La décision concernant le ou les pays dans le(s)quel(s)s demander une protection par brevet dépend avant tout de la stratégie commerciale du demandeur. Le plus souvent, une demande de brevet est déposée auprès du bureau des brevets national de l'inventeur (demandeur) afin de protéger l'invention sur le marché national, puis ensuite elle fait l'objet de dépôts à l'étranger. Toutefois, il n'est pas obligatoire de faire une première demande auprès du bureau des brevets national du demandeur. Il est possible de déposer une demande de brevet auprès de n'importe quel bureau des brevets du monde, sans avoir à déposer préalablement une demande auprès du bureau national de son pays. Aux États-Unis, toutefois, une autorisation de dépôt à l'étranger peut-être exigée avant d'effectuer un dépôt dans un pays étranger.

Le pays dans lequel la première demande est effectuée est appelé le pays de priorité et la date de la première demande est généralement appelée la date de priorité. Les demandes de brevets effectuées auprès d'un bureau des brevets par les résidents de ce pays sont appelés demandes nationales (aux fins statistiques) et les demandes effectuées par des non-résidents sont appelées demandes étrangères. 


\subsubsection{Harmonisation internationale des législations sur les brevets}

Divers traités internationaux ont été établis au fil des ans pour rationaliser la procédure de demande et rendre les procédures de protection par brevet plus efficientes pour les inventeurs (ou les demandeurs) souhaitant une protection dans plusieurs pays. Ces procédures de demande d'examen sont régies par les règles et réglementations du bureau des brevets national (ou régional) et des traités internationaux (tels que la Convention de Paris et le Traité de coopération en matière de brevets), selon le cas.

Les règles applicables aux brevets dans les différents pays ont été considérablement harmonisées dans les années 90, notamment avec l'Accord sur les aspects des droits de propriété intellectuelle qui touchent au commerce (ADPIC) à l'Organisation mondiale du commerce (OMC) (voir encadré 3.2). L'accord ADPIC est un traité international administré par l'OMC qui fixe des normes minimales pour la plupart des formes de réglementation de propriété intellectuelle dans l'ensemble des pays membres de l'OMC. Il a été négocié à la fin de l'Uruguay Round du Traité de l'Accord général sur les tarifs douaniers et le commerce (GATT) en 1994. Il intègre et développe les versions les plus récentes des accords internationaux fondamentaux en matière de propriété intellectuelle administrés par l'OMPI, la Convention de Paris pour la protection de la propriété intellectuelle et la Convention de Berne pour la protection des œuvres littéraires et artistiques, accords qui remontent aux années 1880. Il impose aux pays membres des principes fondamentaux en matière d'échanges internationaux concernant la propriété intellectuelle, notamment le traitement national et celui de la nation la plus favorisée. Les grands changements introduits par l'Accord ADPIC sont notamment les suivants : la durée statutaire des brevets devrait être d'au moins 20 ans après la demande; les brevets devraient couvrir tous les domaines de la technologie (y compris les médicaments, précédemment exclus dans un certain nombre de pays); les brevets devraient être publiés 18 mois après la date de priorité. De nouvelles négociations ont eu lieu dans les années 2000 à l'OMPI et entre pays développés pour poursuivre l'harmonisation des procédures et législations en matière de brevets entre les différents pays, mais cette harmonisation s'est révélée difficile à mener à bien.

\subsubsection{Coûts du dépôt des brevets et durée des procédures}

Une demande de brevet est coûteuse pour le demandeur. Le coût de la protection par brevet peut être subdivisé en quatre grandes catégories, associées au processus de délivrance et au maintien de la protection :

- Frais administratifs : frais d'enregistrement, de recherche, d'examen, de désignation des pays, de délivrance et de publication ainsi que de validation (en Europe). 


\section{Encadré 3.2. Principales dispositions de l'Accord ADPIC}

Les objectifs de l'ADPIC sont définis dans son article 7 : « la protection et le respect des droits de propriété intellectuelle devraient contribuer à la promotion de l'innovation technologique et au transfert et à la diffusion de la technologie, à l'avantage mutuel de ceux qui génèrent et de ceux qui utilisent des connaissances techniques et d'une manière propice au bien-être social et économique, et à assurer un équilibre de droits et d'obligations. " À la différence d'autres accords internationaux sur la propriété intellectuelle, les ADPIC ont introduit un mécanisme de règlement des différends, qui permet des sanctions commerciales contre les États contrevenants. En particulier, les ADPIC abordent la question de l'harmonisation du droit d'auteur et droits connexes, tels que les droits des interprètes ou exécutants, des producteurs de phonogrammes (enregistrements sonores) et des organismes de radiodiffusion; les indications géographiques, notamment les appellations d'origine, les dessins et modèles industriels, les schémas de configuration (topographies) de circuits intégrés; les brevets comprenant la protection de nouvelles variétés de végétaux, les marques de commerce; la présentation commerciale ainsi que les informations non divulguées ou confidentielles, y compris les secrets commerciaux et les données d'essai. Les articles 3 et 4 énoncent les deux principes majeurs de traitement des membres de l'OMC :

- Le traitement national (article 3) : chaque membre accordera aux ressortissants des autres membres un traitement non moins favorable que celui qu'il accorde à ses propres ressortissants en ce qui concerne la protection de la propriété intellectuelle, sous réserve des exceptions déjà prévues dans, respectivement, la Convention de Paris (1967), la Convention de Berne (1971), la Convention de Rome ou le Traité sur la propriété intellectuelle en matière de circuits intégrés.

- Le traitement de la nation la plus favorisée (article 4) : en ce qui concerne la protection de la propriété intellectuelle, tous avantages, faveurs, privilèges ou immunités accordés par un membre aux ressortissants de tout autre pays seront, immédiatement et sans conditions, étendus aux ressortissants de tous les autres membres.

- Coûts de traitement : coûts associés à la rédaction de la demande et au suivi de la procédure (contacts avec les examinateurs et le bureau des brevets), supportés par le demandeur. Ces coûts peuvent être internes (service de l'entreprise s'occupant de la propriété intellectuelle) ou externalisés (conseil privé en brevets).

- Coûts de traduction, en cas de demande à l'étranger. Ces coûts sont principalement encourus après l'octroi du brevet et ils sont fonction de la 
longueur du texte du brevet. Plus les pays couverts sont nombreux, plus les coûts de traduction sont élevés.

- Les coûts de maintien en vigueur sont les coûts de renouvellement encourus pour maintenir la validité du brevet pour une durée maximale de 20 ans, auxquels peuvent s'ajouter des commissions à verser aux conseils en brevets servant d'intermédiaire entre le détenteur du brevet et le bureau des brevets ${ }^{4}$. Les frais de renouvellement varient fortement d'un pays à l'autre.

Il existe également d'autres coûts liés à la protection du brevet, c'est-àdire à la défense des droits qui s'y attachent par la recherche et la répression des cas de violation (par exemple, par des poursuites judiciaires) ou à l'invalidation ou l'opposition par des tiers, etc. Le calcul des coûts liés aux brevets est une tâche complexe, car plusieurs éléments ne sont pas faciles à chiffrer et dépendent des motivations du demandeur pour le dépôt du brevet. Plusieurs facteurs conditionnent le coût total du brevet (par exemple, le nombre de revendications, le nombre de pages, la voie empruntée, la qualité des services externes, la rapidité souhaitée et l'étendue géographique de la protection). Les brevets les plus importants (c'est-à-dire ceux qui présentent le plus de revendications ou comptent le plus de pages) et les brevets destinés à être déposés dans un grand nombre de pays membres de la CBE sont plus coûteux en termes à la fois de coûts de procédure et de coûts externes. Le coût est par ailleurs lié à la durée de la procédure (notamment lorsqu'il existe une communication écrite abondante entre le conseil en brevet et le bureau des brevets), de même qu'à la rapidité souhaitée du processus de délivrance. Compte tenu de l'extrême variabilité des coûts suivant les domaines techniques et les pays, il est difficile de donner des chiffres moyens significatifs sur le coût du dépôt des brevets. De plus, ces coûts doivent être mis en correspondance avec la taille du marché couvert (c'est-à-dire le marché potentiel sur lequel l'exclusivité est demandée pour l'invention).

Une enquête auprès des demandeurs de brevets réalisée en 2004 a porté sur le coût des brevets (OEB, Roland Berger, 2005). Le coût d'obtention d'un brevet standard Euro-direct (dépôt direct auprès de l'OEB ou extension d'une demande antérieure de brevet national) en 2003 a été estimé à 30530 EUR (OEB et Roland Berger Market Research) tandis que les coûts (estimés) d'un brevet Euro-PCT (dépôt par procédure PCT auprès de l'OMPI désignant l'OEB) s'élevaient en moyenne à quelque $46700 \mathrm{EUR}^{5}$. La différence par rapport aux demandes de brevets Euro-direct vient principalement des coûts de traduction plus élevés en raison d'un plus grand nombre de pages (description et revendications), des redevances officielles supplémentaires liées à la phase internationale, et de la validation dans un plus grand nombre de pays (huit au lieu de six). Une entreprise d'un pays européen (État membre de l'OEB) acquittera en moyenne 24100 EUR pour se voir accorder et valider un brevet Euro-direct; une entreprise américaine paiera 10250 EUR pour recevoir un 
brevet de l'USPTO; une entreprise japonaise paiera 5460 EUR pour obtenir la délivrance d'un brevet par le JPO. Les coûts plus élevés en Europe tiennent principalement aux coûts de traduction dans les phases de traitement et de validation. Bien qu'elles varient selon les bureaux des brevets, les taxes officielles jouent un rôle mineur dans la différence totale: les chiffres indiqués par les demandeurs sont de 3470 EUR à l'OEB, de 2050 EUR à l'USPTO et de 1570 EUR au JPO.

La durée des procédures est également extrêmement variable selon les bureaux des brevets et elle a évolué au fil du temps. Le délai moyen d'examen (temps écoulé entre le dépôt et la délivrance) à l'OEB était d'environ 44 mois en 2006; elle aurait augmenté de $8 \%$ par rapport à 2005. Au JPO, le délai restait stable à environ 31.8 mois alors qu'à l'USPTO il fallait en moyenne 31.3 mois en 2006 comparé a 30.6 mois en 2005 (Trilateral Statistical Report, 2006). L'augmentation du délai soulève des problèmes statistiques particuliers. Par exemple, les statistiques annuelles sur les demandes et les délivrances sont de plus en plus découplées; les statistiques sur les procédures (taux de délivrance, de refus, de retrait) sont faussées à la longue et il devient difficile d'interpréter les tendances.

Tous les stades de la procédure de protection par brevet génèrent d'importants volumes d'information sur l'invention pour laquelle une protection est demandée. Les informations sur la phase de procédure des demandes de brevets apportent des éclairages sur la stratégie du demandeur, mais elles soulèvent aussi des difficultés statistiques.

- Premièrement, aucune statistique n'est disponible avant 18 mois après la date de priorité, car la demande n'est pas publiée avant cette date ${ }^{6}$. C'est un problème pour les analystes, car cela limite l'actualité légalement possible des données sur les brevets.

- Le rapport de recherche contient des informations précieuses telles que les références à l'état antérieur de la technique (références par rapport ou non à des brevets), qui peuvent être considérées comme des précédents de l'invention couverte par le brevet.

- La liste des pays dans lesquels la demande est soumise, ou le fait d'emprunter la voie internationale (PCT) est une indication de la stratégie commerciale du demandeur (locale, régionale ou mondiale). On peut également se faire ainsi une idée de la valeur de l'invention, dans la mesure où on peut considérer que le revenu escompté de l'invention brevetée sera supérieur au coût prospectif du brevetage.

- La durée de la procédure de demande de brevet (temps nécessaire pour que le bureau des brevets parvienne à une décision) est révélatrice à la fois de la stratégie du demandeur (qui peut soit rechercher une délivrance rapide, soit vouloir prolonger la procédure) et de l'efficience du bureau des brevets 
(capacité à gérer sa charge de travail). Le fait qu'une demande soit accordée ou refusée donne une indication de sa qualité.

\subsection{Procédures nationales et régionales}

Tous les bureaux des brevets ont leurs statuts particuliers, qui peuvent présenter des variations par rapport au modèle de base décrit plus haut. Les différences peuvent résider dans le "droit matériel des brevets " (ce qui est brevetable ou pas, etc.) ou dans les procédures elles-mêmes, bien que la distinction entre ces deux aspects ne soit pas toujours tranchée. Les procédures les plus spécifiques sont celles de l'OEB, car il s'agit d'un bureau des brevets non pas national, mais régional et international. Le tableau 3.1 résume certaines des différences dans les règles appliquées par les trois grands bureaux. Des négociations actives au niveau international visent à éliminer ces différences dans l'avenir.

La procédure de délivrance n'est pas identique dans tous ces bureaux. Ainsi, l'examen à l'OEB se fait en deux phases (recherche et examen sur le fond $^{7}$ ) alors que dans les procédures nationales devant le JPO et l'USPTO, ces deux phases sont regroupées. Après l'examen, le bureau des brevets informe le demandeur de sa décision (OEB : avis de délivrance; JPO : décision de délivrance; USPTO : avis d'autorisation). Si un brevet ne peut être délivré sous la forme dans laquelle il a été soumis, l'intention de rejeter la demande est communiquée (OEB : rapport d'examen; JPO : notification de la raison du refus; USPTO : décision administrative de rejet). Le demandeur peut alors modifier sa demande, notamment en ce qui concerne les revendications, après quoi l'examen reprend. Cette phase procédurale dure tant que le demandeur continue d'apporter les modifications adéquates. Puis, soit le brevet est accordé, soit la demande est finalement rejetée ou retirée par le demandeur. Dans les trois bureaux des brevets, le demandeur peut retirer ou abandonner la demande à tout moment avant que celle-ci ne soit déclarée recevable ou finalement rejetée. Dans la section ci-après sont décrites plus en détail certaines des différences entre les bureaux des brevets qui doivent être prises en compte dans le calcul de statistiques sur les brevets.

\subsubsection{USPTO}

Aux États-Unis, la Constitution donne au Congrès le pouvoir de promulguer des lois pour " promouvoir le progrès des sciences et des arts utiles... ». Les lois promulguées par le Congrès au sujet du système de brevets ont été codifiées dans le titre 35 du Code des États-Unis et ont porté création de l'United States Patent \& Trademark Office. 


\section{Tableau 3.1. Différences entre les trois principaux bureaux des brevets}

\begin{tabular}{|c|c|c|c|}
\hline & OEB & JPO & USPTO \\
\hline Le brevet est délivré au : & Premier demandeur & Premier demandeur & Premier inventeur \\
\hline Durée du brevet : & 20 ans & 20 ans & 20 ans \\
\hline Langue de la demande: & $\begin{array}{l}\text { Anglais, français } \\
\text { ou allemand }\end{array}$ & Japonais $^{b}$ & Anglais $^{C}$ \\
\hline Région couverte: & $\begin{array}{l}\text { Membres de la CBE } \\
\text { et «pays d'extension » } d\end{array}$ & Japon & États-Unis \\
\hline Demande d'examen: & Oui, dans les 6 mois & Oui, dans les 3 ans $^{e}$ & Non \\
\hline $\begin{array}{l}\text { Publication } \\
\text { de la demande : }\end{array}$ & $\begin{array}{l}18 \text { mois après la date } \\
\text { de priorité }\end{array}$ & $\begin{array}{l}18 \text { mois après la date } \\
\text { de priorité }\end{array}$ & $\begin{array}{l}18 \text { mois après la date } \\
\text { de prioritéf }^{f}\end{array}$ \\
\hline $\begin{array}{l}\text { Certains éléments sont-ils } \\
\text { considérés comme non } \\
\text { brevetable ou ne pouvant } \\
\text { faire l'objet d'inventions? }\end{array}$ & $\mathrm{Oui}^{g}$ & Oui $^{h}$ & Oui $^{i}$ \\
\hline Système d'opposition : & Oui ${ }^{j}$ & Non & Non $^{k}$ \\
\hline
\end{tabular}

a) La demande peut être présentée dans n'importe quelle langue officielle de n'importe quel pays partie à la CBE. Toutefois, dans les trois mois suivant la demande et au plus tard 13 mois après la toute première date de priorité, une traduction de la demande est exigée dans l'une des langues officielles de l'OEB (anglais, français ou allemand).

b) Il est possible de présenter une demande de brevet en japonais, les spécifications, revendications, dessins et résumé étant en anglais. Une traduction en japonais des documents en anglais doit être présentée dans les 14 mois suivant la date de demande initiale.

c) Il est possible de présenter une demande dans n'importe quelle langue autre que l'anglais, à condition qu'une traduction en anglais soit communiquée dans les deux mois.

d) Un brevet européen n'assure pas automatiquement une protection dans l'ensemble des pays membres parties à la CBE (ou dans les pays " d'extension "). Le demandeur doit valider son brevet OEB, une fois celui-ci délivré, dans chacun des bureaux nationaux de brevets, pour que son brevet soit reconnu dans les pays correspondants.

e) Délai pour la demande d'examen : trois ans pour les brevets dont la demande est postérieure à octobre 2001 et sept ans pour ceux dont la demande est antérieure à octobre 2001.

f) Une demande qui n'a pas fait et ne fera pas l'objet d'une demande à l'étranger peut ne pas être publiée, si tel est le souhait du demandeur.

g) Les éléments qui en sont pas considérés comme pouvant faire l'objet d'inventions sont les suivants : découvertes, théories scientifiques et méthodes mathématiques; créations esthétiques; schémas, règles et méthodes pour la réalisation d'actes mentaux, jouer à des jeux ou faire des affaires, et programmes d'ordinateurs et présentations d'informations. Éléments exclus de la brevetabilité : végétaux et animaux; et les méthodes de traitement chirurgical ou thérapeutique du corps humain ou animal et les méthodes de diagnostic appliquées au corps humain ou animal.

h) Les éléments qui ne sont pas considérés comme des inventions sont les suivants : découvertes; théories scientifiques et méthodes mathématiques; activités mentales; simple présentation d'informations; méthodes commerciales; parties isolées d'êtres humains; et méthodes diagnostiques, thérapeutiques et chirurgicales de traitement humain ou animal.

i) Les éléments qui ne sont pas considérés comme des inventions sont les suivants: théories scientifiques et méthodes mathématiques « abstraits "; actes mentaux; présentation de l'information; et connaissances traditionnelles.

j) Dans les neuf mois suivant la publication de la mention de délivrance d'un brevet européen, toute personne peut signifier à l'OEB son opposition au brevet européen délivré. L'opposition n'est possible que pour les motifs suivants : l'objet du brevet n'est pas brevetable; le brevet ne décrit pas l'invention de façon claire et complète; ou l'objet du brevet dépasse le champ défini dans la demande.

k) Procédure de réexamen; examen après délivrance de la validité des revendications d'un brevet compte tenu de l'état antérieur de la technique ou de littérature hors brevets qui pourraient avoir une incidence sur la brevetabilité d'une quelconque revendication du brevet en question. Le détenteur du brevet ou n'importe quel tiers peut demander à tout moment un réexamen après la délivrance du brevet. 
L'USPTO se caractérise par les différences suivantes par rapport à la procédure standard de demande de brevet, qui sont liées aux spécificités du système de brevets américain :

- Les États-Unis accordent un brevet au premier inventeur et non au " premier à déposer une demande " comme dans tous les autres pays. Cela signifie que le premier à déposer une demande peut voir ce droit contesté devant l'USPTO par une autre partie prétendant avoir réalisé antérieurement l'invention, même s'il n'a pas présenté de demande de brevet (demande de brevet tardive).

- Les États-Unis appliquent une période de grâce pour l'évaluation du caractère novateur. Les publications (par exemple dans des revues universitaires) par l'inventeur durant la période de grâce, qui peut s'étaler jusqu'à un an avant la date de dépôt de la demande, ne sont pas prises en compte pour déterminer le caractère novateur de l'invention.

- La durée statutaire des brevets est de 20 ans à compter de la demande depuis 1995 (date à laquelle les États-Unis ont transposé les ADPIC dans leur législation nationale) alors que précédemment elle était de 17 ans suivant la délivrance ${ }^{8}$. Les droits de maintien en vigueur doivent être acquittés 3.5, 7 et 11.5 années après l'octroi du brevet (ils sont annuels dans la plupart des autres pays).

- Une demande auprès de l'USPTO est automatiquement considérée comme une demande d'examen (à la différence de la plupart des autres pays, où le demandeur dispose d'une certaine période après réception du rapport de recherche avant de décider de présenter ou non une demande d'examen; voir la procédure concernant le brevet européen). Cela signifie en particulier que les demandeurs devront se soumettre à l'examen, même s'ils réalisent après la recherche que le caractère novateur de leur invention n'est pas garanti. Toutefois, un nombre croissant de demandes auprès de l'USPTO emprunte la voie du PCT, dans laquelle cette règle ne s'applique pas.

- Jusqu'à récemment, les brevets américains n'étaient publiés qu'après avoir été octroyés. Cela a changé et maintenant les demandes de brevets américains sont publiées 18 mois après la date de dépôt de la demande à moins qu'ils aient été retirés ou que leur dépôt s'accompagne d'une demande de nonpublication (si le demandeur déclare ne pas déposer une demande annexe [dans un autre pays] faisant référence à la priorité de la première demande auprès de l'USPTO).

- Lorsqu'ils soumettent une demande de brevet, les demandeurs (ou inventeurs) sont invités à fournis une liste de l'état antérieur de la technique. Contrairement à la procédure à l'OEB, un "devoir de sincérité " s'impose à quiconque intervient dans une demande de brevet américain, depuis l'inventeur jusqu'au conseil en brevet, pour attirer l'attention de 
l'USPTO sur tout art antérieur dont l'inventeur (de même que tous les autres intervenants dans la demande de brevet, tels que le conseil en brevet) a connaissance ou prend connaissance et qui pourrait avoir une importance pour la brevetabilité. Il s'agit d'une obligation légale et son non-respect par le demandeur d'un brevet peut conduire à la révocation ultérieure du brevet. Cela a conduit à une inflation de mentions de l'état antérieur de la technique, et amené l'USPTO à réagir en 2005 en encourageant les demandeurs à limiter le nombre de références soumises à 25 . Ces différences institutionnelles expliquent en partie pourquoi les citations sont sensiblement plus nombreuses dans les brevets de l'USPTO que dans les brevets émanant des autres bureaux (tableau 3.1).

- Depuis le 8 juin 1995, l'USPTO offre aux inventeurs la possibilité de déposer une demande provisoire de brevets dont la finalité est d'offrir un moyen à moindre coût de déposer une première demande de brevet aux États-Unis. Il s'agit d'une demande de brevet qui ne débouche pas sur la délivrance d'un brevet à moins que d'autres initiatives ne soient prises par le demandeur. Une demande provisoire permet le dépôt d'une demande sans revendication officielle de brevet ou la communication d'informations (état antérieur de la technique). C'est un moyen d'établir très tôt une date de dépôt effective dans une ou plusieurs demandes de brevet en cours, pour revendiquer ultérieurement la date de priorité de l'invention divulguée dans des demandes provisoires antérieures déposées par le ou les même(s) inventeur(s) ${ }^{9}$.

- Les demandeurs ont la possibilité, après la demande, de modifier de façon assez sensible leur demande initiale, en fonction des progrès de leurs recherches ou en réaction aux demandes des examinateurs. Cette phase procédurale se répète tant que le demandeur continue d'apporter les amendements appropriés; en conséquence, la délivrance peut être retardée. Il y a continuation partielle (continuation-in-part - CIP) en cas de dépôt d'une deuxième demande puis de demandes ultérieures, impliquant la protection de nouveaux éléments, alors que la demande originale est en cours.

- S'il apparaît qu'un brevet a été délivré à tort, le détenteur de brevet peut renoncer à celui-ci et soumettre de nouveau la demande originale pour remédier au problème. Il arrive ainsi fréquemment que le brevet délivré ne couvre pas tout le champ de l'invention. Ainsi, un inventeur peut soumettre de nouveau la demande de brevet avec des revendications plus larges et/ou nouvelles et essayer d'obtenir l'ensemble de la couverture auquel celles-ci peuvent prétendre. Il n'est toutefois pas autorisé à ajouter de nouvelles caractéristiques à son invention. La demande de redélivrance pour tenter d'obtenir une couverture plus large que celle offerte dans le brevet délivré au départ doit être déposée dans les deux années suivant la date initiale de délivrance du brevet. 


\subsubsection{JPO}

La législation japonaise sur les brevets a été réformée à plusieurs reprises depuis la fin des années 80, pour la rapprocher de celle des autres pays. Les principales spécificités ayant des incidences dans le domaine statistique sont les suivantes :

- Le JPO délivre des brevets sur la base du premier déposant, c'est-à-dire selon le principe que lorsque deux parties demandent un brevet pour une même invention, le brevet ira au premier à avoir déposé une demande.

- Le Japon applique également une période de grâce. Jusqu'à six mois avant le dépôt de la demande, une invention qui a été publiée ou présentée devant un organisme universitaire désigné par le Commissaire, ou a été présentée dans un salon organisé par un gouvernement ou un organisme désigné par le Commissaire n'est pas considérée comme ayant perdu son caractère novateur.

- Le JPO publie le contenu de la demande au Bulletin officiel lorsque 18 mois se sont écoulés depuis la date de priorité ${ }^{10}$. Toutefois, une demande d'examen doit être présentée dans les trois ans suivant la date de demande pour lancer le processus d'examen sur le fond. Le délai maximal pour la demande d'examen a été ramené en 2001 de sept à trois ans (trois ans pour les brevets déposés depuis octobre 2001 et sept ans pour les brevets déposés avant octobre 2001). Si le demandeur ne demande pas l'examen dans le délai fixé, la demande est considérée comme retirée.

- Le long délai accordé aux demandeurs pour décider ou non de demander un examen pourrait être l'une des raisons pour lesquelles les demandes auprès du JPO sont nombreuses comparées à d'autres juridictions, dans la mesure où les inventeurs peuvent attendre plus de huit ans avant de prendre une décision. Cette nouvelle règle explique également le gonflement du nombre des examens demandés (et des délivrances) après 2004, par un effet de calendrier. Ce nombre élevé peut aussi s'expliquer par la règle d'une revendication unique qui prévalait au Japon jusqu'en 1975. L'actuelle unité de demande équivaut à l'unité d'invention dans les autres juridictions (telle que définie dans le PCT). Cela permet en substance à des groupes d'inventions liées de former un seul et même concept inventif examiné dans le cadre d'une seule et même demande. Malgré ces réformes, les demandes auprès du JPO comportent toujours un nombre sensiblement plus faible de revendications que dans les autres bureaux des brevets. Un inventeur peut devoir soumettre plusieurs demandes au JPO au lieu d'une seule auprès d'autres bureaux pour obtenir le même niveau de protection. Toutefois, comme les demandeurs s'efforcent d'obtenir des droits étendus et solides sur leurs technologies, le nombre de revendications par demande augmente depuis la fin des années 80. 
- Au JPO, les frais de maintien en vigueur du brevet sont exigibles sous la forme d'une somme forfaitaire pour les trois premières années, puis par la suite annuellement à compter de la quatrième année de la date de délivrance. L'obligation pour les demandeurs de communiquer des informations sur l'état antérieur de la technique dans les demandes a été introduite au $1^{\mathrm{er}}$ septembre 2002 et est entrée en vigueur le $1^{\mathrm{er}}$ mai 2006. Les examinateurs de brevets procèdent à une recherche de l'état antérieur de la technique. Il n'y a pas de limite quant au nombre de références pouvant être incluses.

- Les brevets accordés par le JPO peuvent faire l'objet de contestations par des tiers. Même après qu'un brevet a été enregistré, n'importe qui peut demander l'invalidation de celui-ci s'il présente un problème. Ce système a été introduit en 2003 , date à laquelle le système d'opposition après délivrance a été aboli et le système expérimental d'invalidation a été révisé (il est en vigueur depuis le $1^{\mathrm{er}}$ janvier 2004). Dans la nouvelle procédure expérimentale d'invalidation : i) le jugement peut être demandé à tout moment; ii) les deux parties sont impliquées dans une procédure inter partes durant le procès; et iii) le plaignant peut faire appel du verdict confirmant le brevet en question auprès du Tribunal suprême de Tokyo.

\subsubsection{OEB}

La Convention sur la délivrance de brevets européens, plus connue sous le nom de Convention sur le brevet européen (CBE) a été signée en 1973 et est entrée en vigueur en 1977. Dans le prolongement de la CBE, l'Office européen des brevets $(\mathrm{OEB})^{11}$ a été créé pour délivrer des brevets européens fondés sur une procédure d'examen centralisée. En déposant une demande unique de brevet européen dans l'une des trois langues officielles (anglais, français et allemand), il est possible d'obtenir des droits de brevets dans l'ensemble des pays membres à la $\mathrm{CBE}^{12}$ :

- Les brevets délivrés par l'OEB assurent les mêmes droits légaux et sont soumis aux mêmes conditions que les brevets nationaux (délivrés par les bureaux des brevets nationaux) dans chaque pays partie à la CBE pour lequel le brevet a été accordé. Une fois accordé par l'OEB, un brevet européen est donc un « paquet » de brevets nationaux, qui doivent être validés dans les bureaux des brevets nationaux des États désignés pour qu'ils deviennent effectifs dans les pays membres de la $\mathrm{CBE}^{13}$. Dans les trois mois suivant la délivrance d'un brevet européen, le demandeur doit compléter diverses formalités. Ainsi, le bureau des brevets national d'un État désigné peut demander au demandeur de fournir une traduction dans l'une de ses langues officielles et d'acquitter les droits de publication du brevet.

- Une demande de brevet européen peut émaner : i) d'une demande directe auprès de l'OEB sans revendication de priorité (c'est-à-dire première 
demande); ii) de l'extension d'une demande antérieure de brevet national (dans les 12 mois de la première demande); ou iii) d'une demande internationale déposée selon la procédure PCT. Les deux premières catégories sont appelées « Euro-direct », tandis que la troisième est appelée " Euro-PCT ». Le graphique 3.2 illustre ces trois voies différentes de demande de brevet auprès de l'OEB. Depuis le début des années 2000, la part des demandes de brevets émanant des bureaux nationaux adressées à l'OEB ont sensiblement diminué au fil du temps. De fait, la majorité des demandes de brevets à l'OEB ont emprunté la voie PCT (Euro-PCT). En 2006, la proportion de l'ensemble des demandes PCT entrant dans la phase nationale-régionale était de $62 \%$ à l'OEB, alors qu'elle était de $46 \%$ à l'USPTO et 455 au JPO (Trilateral Statistical Report, 2006) ${ }^{14}$. La répartition est similaire en ce qui concerne la part du PCT dans le total des brevets délivrés par les bureaux des brevets trilatéraux: en 2006, 52 \% des brevets délivrés par l'OEB étaient des demandes PCT, contre $11 \%$ à l'USPTO et $5.1 \%$ au JPO.

- Ce contexte juridique complexe est une source de difficultés statistiques, notamment pour le comptage des "brevets nationaux » et des « demandes nationales " dans les pays européens. Strictement parlant, toutes les demandes auprès de l'OEB depuis 2004 sont également des demandes nationales, dans la mesure où le demandeur a le droit, en cas de délivrance, d'obtenir un brevet dans le pays concerné. Cela vaut également si le demandeur n'a pas l'intention de solliciter une protection dans ce pays, comme cela est le plus souvent le cas pour les petits pays européens. En conséquence, la notion de "demande de brevet nationale » est floue. Cela n'est toutefois pas propre aux pays européens, un principe similaire de désignation automatique étant maintenant en place dans le cadre du PCT (section 3.4.2). De ce fait, si l'on souhaite compiler des statistiques exhaustives sur les demandes nationales dans un pays donné, il faut utiliser ensemble les données nationales, les données OEB et les données PCT. De plus, en Europe, les brevets valides dans un pays quelconque comprennent non seulement ceux examinés et délivrés par le bureau national des brevets, mais également ceux délivrés par l'OEB et validés au plan national.

Parmi les autres particularités de la procédure OEB, on peut noter :

- Contrairement à ce qui se passe à l'USPTO, la communication de références sur l'état antérieur de la technique lors du dépôt d'une demande est facultative. C'est aux examinateurs qu'il revient d'établir une liste de références sur l'état antérieur de la technique (mentionnées dans le rapport de recherche), en regard desquelles la brevetabilité est évaluée. Le rapport de recherche européen devrait comprendre comme référence les documents les plus importants ou les premières publications de documents d'importance équivalente. Selon la philosophie de l'OEB, un bon rapport de recherche contient l'ensemble des informations pertinentes, avec un nombre minimum de citations. 
- Une fois publié le rapport de recherche européen, le demandeur a six mois pour déposer une demande d'examen et acquitter les droits correspondants; sinon, la demande est considérée comme retirée.

- Une opposition à un brevet délivré par l'OEB peut être déposée par des tiers dans un délai de neuf mois suivant la date de délivrance. Cette procédure est une source intéressante de données statistiques. L'opposition étant une procédure coûteuse, il est probable que les brevets faisant l'objet d'une opposition sont ceux qui créent le plus de difficultés (coût économique potentiel) pour les concurrents, et que ce sont donc ceux ayant la plus forte valeur. Le fait qu'un brevet fasse l'objet d'une opposition peut donc être un signe de sa valeur élevée (Harhoff et Reitzig, 2002).

\subsection{Demande internationale de brevets}

\subsubsection{Le principe de priorité}

Le plus ancien traité international sur la protection des inventions remonte à 1883 (Convention de Paris sur la protection de la propriété intellectuelle), et il comptait 169 pays signataires en janvier 2005. La Convention de Paris a mis en place un système de droits de priorité, dans lequel les demandeurs ont jusqu'à 12 mois à compter de la date de leur première demande de brevet (en général, dans leur propre pays) pour déposer des demandes ultérieurement dans d'autres pays signataires, en revendiquant la date de priorité de la première demande. Avant la Convention de Paris, les demandes étrangères pouvaient être refusées au motif que l'invention n'avait plus de caractère de nouveauté car celleci avait été rendue publique dans une demande (prioritaire) antérieure ${ }^{15}$.

La règle des droits de priorité a d'importantes conséquences pour le calcul des statistiques sur les brevets, car dans la plupart des pays, il existera un décalage de 12 mois entre les dates de demande intérieure et de demande à l'étranger pour une invention donnée. Cela veut dire que pour une demande nationale, la date de priorité équivaut à la date de dépôt de la demande et que pour les demandes à l'étranger, il existe un décalage de 12 mois entre la date de priorité et la date de dépôt de la demande. Si l'on utilise la date de dépôt de la demande pour situer l'invention dans le temps, cela introduira un biais dans la chronologie des inventions nationales et étrangères. La date de priorité en revanche rendra compte de la bonne chronologie de la découverte des inventions tant nationales qu'étrangères. C'est la raison pour laquelle dans la compilation de statistiques des brevets destinées à rendre compte des activités d'invention, il est recommandé d'utiliser la priorité comme date de référence.

\subsubsection{Le Traité de coopération en matière de brevets}

Le Traité de coopération en matière de brevets (PCT) a été signé en 1970 et est entré en vigueur en 1978. Il est administré par l'Organisation mondiale de 
la propriété intellectuelle (OMPI). Au 31 juillet 2006, on dénombrait 133 États parties à ce traité. Le PCT ne délivre pas de brevets. La procédure PCT permet de demander des droits de brevet dans un grand nombre de pays en soumettant une demande internationale unique (demande PCT) auprès d'un seul bureau des brevets (bureau de réception), puis, lors de la phase nationale, dans les pays souhaités à un stade ultérieur ${ }^{16}$. Toutes les demandes (internationales ou régionales) doivent, au bout du compte, avoir un statut national, c'est-à-dire qu'elles doivent être validées (délivrées) par les bureaux des brevets nationaux des pays dans lesquels une protection est demandée.

Concrètement, la procédure PCT donne aux demandeurs la possibilité de retarder les procédures nationales ou régionales et donc de retarder les taxes et coûts de traduction correspondants pour une durée maximale de 30 mois suivant la date de priorité. Le demandeur peut donc bénéficier de davantage d'informations (concernant la valeur potentielle du brevet) avant de s'engager dans les coûts élevés du dépôt de demandes auprès d'un grand nombre de bureaux nationaux. En ce sens, une demande PCT peut être considérée comme une option sur des demandes futures auprès de bureaux des brevets dans le monde.

La demande PCT débute par le dépôt d'une demande internationale auprès soit du bureau des brevets national (ou régional) soit de l'OMPI. Celle-ci doit être faite dans la période de 12 mois suivant la demande prioritaire, mais elle peut être effectuée immédiatement pour faire office de demande prioritaire (graphique 3.1). Le demandeur doit être un ressortissant ou un résident de l'un des pays signataires du PCT. Une demande PCT englobe automatiquement tous les États signataires du traité comme États désignés (qui sont les pays dans lesquels le demandeur souhaite protéger son invention) ${ }^{17}$.

\section{Graphique 3.1. Chronologie des procédures PCT}

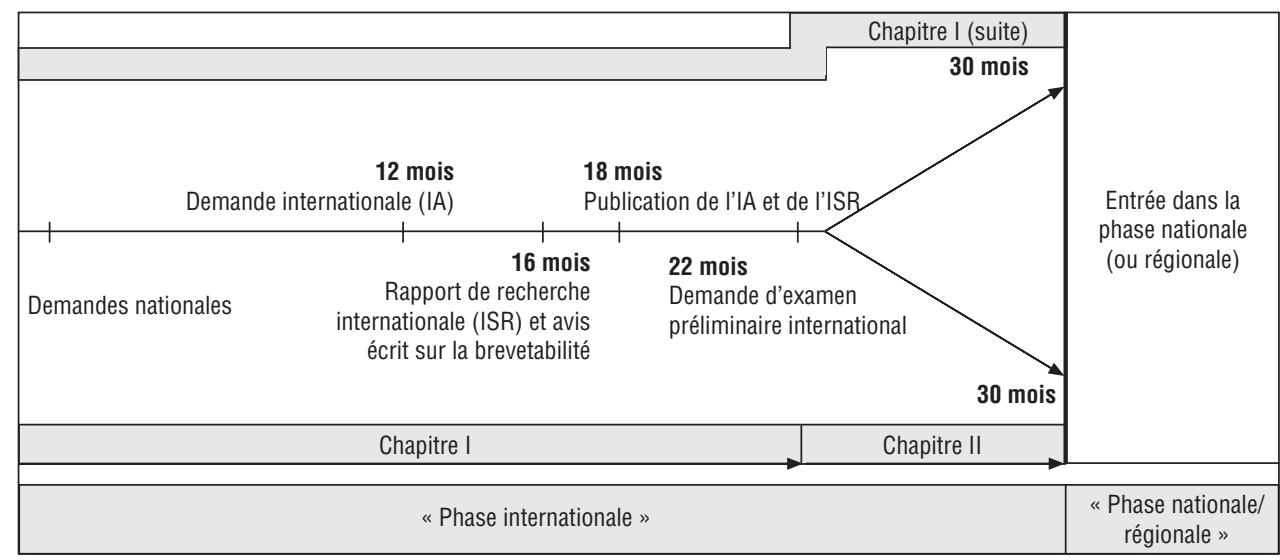


Une fois reçue à l'OMPI, la demande est transmise à l'autorité chargée de la recherche internationale désignée, qui est un bureau des brevets nommé par l'OMPI (ce peut être par exemple l'OEB, le JPO ou l'USPTO). L'administration chargée de la recherche internationale prépare un rapport de recherche internationale qui est publié en même temps que la demande. Il est construit de la même manière que les rapports de recherche dans les procédures nationales. Celui-ci répertorie les références aux documents de brevet publiés et articles de revues techniques susceptibles d'influer sur la brevetabilité de l'invention. Le rapport de recherche internationale est normalement communiqué par l'administration chargée de la recherche internationale au demandeur neuf mois après le dépôt de la demande en cas de premier dépôt et 16 mois après la date de priorité en cas de dépôt ultérieur (c'est-à-dire revendication de la priorité d'une première demande). Outre le rapport de recherche internationale, depuis janvier 2004, un avis écrit détaillé sur la brevetabilité de l'invention revendiquée est communiqué (WOISA). Le WOISA est un avis non contraignant sur le fait que l'invention semble ou non remplir les critères de brevetabilité compte tenu des résultats du rapport de recherche. La demande internationale et le rapport de recherche internationale sont publiés 12 mois après la date de priorité (les opinions écrites ne sont pas publiées).

Lorsqu'il a reçu le rapport de recherche internationale et le WOISA, le demandeur peut également solliciter un examen préliminaire international, qui conduira à un rapport préliminaire international sur la brevetabilité. Ce rapport est une deuxième évaluation de la brevetabilité potentielle de l'invention. La demande d'examen préliminaire internationale doit être déposée dans les 22 mois suivant la date de priorité (ou dans les trois mois suivant la publication du rapport de recherche internationale, si cette date vient après). Si le demandeur ne sollicite pas un examen préliminaire international, le WOISA peut être converti en un rapport préliminaire international sur la brevetabilité ${ }^{18}$. Enfin, au bout de 30 mois suivant la date de priorité, la phase internationale s'achève et la demande internationale entre dans la phase nationale ou régionale dans laquelle le demandeur souhaite effectivement demander un brevet ${ }^{19}$. On se souviendra que toutes les demandes internationales ou régionales doivent en définitive avoir un statut national.

S'agissant du PCT, il convient de noter qu'après le passage à la phase nationale ou régionale, il faut environ six mois supplémentaires pour que cette étape soit publiée dans le bureau régional/national. Dans le cas des demandes Euro-PCT, l'information sur le transfert effectif à l'OEB est disponible 36 mois après la date de priorité (première demande). La disponibilité tardive de cette information influe beaucoup sur le calcul de statistiques sur les brevets et sur l'actualité des indicateurs sur les brevets dans les bureaux des brevets nationaux ${ }^{20}$. Dans le chapitre suivant, on traitera la question de l'actualité des données et présentera rapidement diverses méthodes de « rétropolation » des demandes de brevets. 


\section{Notes}

1. De façon générale, il existe une période d'attente entre la demande d'examen et la première décision du bureau, telle que le premier avis de refus ou la décision de délivrance. Au JPO, la période d'attente moyenne était de 25.8 mois en 2005 tandis qu'à l'OEB elle était de 23.8 mois et de 23.4 à l'USPTO (Trilateral Statistical Report, 2006).

2. Aucun rapport de recherche n'est communiqué dans les publications avant délivrance de l'USPTO ou dans les demandes de brevets auprès du JPO.

3. De nombreuses juridictions accordent une couverture plus longue pour les médicaments afin de compenser les retards administratifs dans l'octroi de l'approbation de mise sur le marché.

4. Ces droits sont exigibles chaque année par les bureaux des brevets nationaux des pays membres parties à la CBE ou après trois, sept et onze ans à l'USPTO. Ces droits augmentent généralement progressivement au fil du temps. Lorsqu'un brevet est délivré par l'OEB, celui-ci doit être validé dans chacun des bureaux des brevets nationaux souhaités des pays membres partie à la CBE. Au JPO, les droits de renouvellement sont exigibles sous la forme d'un montant forfaitaire pour les trois premières années, puis ensuite annuellement à partir du quatrième anniversaire de la date de délivrance.

5. Ce chiffre comprend les taxes dues au titre de la procédure de délivrance à l'OEB, les coûts de représentation par un conseil en brevet devant l'OEB, les coûts de traduction et de validation ainsi que les taxes annuelles pour le maintien en vigueur du brevet.

6. Les bureaux des brevets établissent des comptages agrégés des demandes récentes pour le suivi de leurs propres activités, mais ces données ne sont pas accessibles aux utilisateurs extérieurs et ne peuvent être exploitées à des fins analytiques.

7. Tout d'abord, une recherche est effectuée pour établir l'état antérieur de la technique en regard de l'invention. Le demandeur reçoit un rapport de recherche accompagné d'une première opinion sur la brevetabilité. Dans une deuxième phase, un examen sur le fond vise les critères d'inventivité et d'applicabilité industrielle.

8. Les brevets dont la demande était antérieure au 8 juin 1995, et qui étaient ou seront en vigueur après le 8 juin 1995 ont une durée de validité de 17 ans à compter de la date de délivrance du brevet ou de 20 ans à compter de la date de dépôt de la plus ancienne demande en relation avec les brevets, si celle-ci assure une protection plus longue.

9. Comme la demande ne fait l'objet d'un examen de la brevetabilité par rapport à l'état antérieur de la technique, la redevance perçue par l'USPTO pour le dépôt d'une demande de brevet provisoire est sensiblement plus faible que celle requise pour le dépôt d'une demande de brevet standard non provisoire.

10. Au JPO, depuis 2000, il est possible de demander la publication rapide de la demande de brevet dans un délai d'un an et demi suivant la date de dépôt de la demande afin de dissuader toute imitation par des tiers, un dédommagement pouvant être exigé en cas de violation à compter de la date de publication.

11. L'OEB n'est pas une institution de l'Union européenne. Actuellement, il n'existe pas de brevet unique communautaire, bien que depuis les années 70 le débat se poursuive sur la création d'un "brevet communautaire " au sein de l'Union européenne. Dans sa communication au Parlement européen et au Conseil (3 avril 2007, COM, 165 Final) "Améliorer le système de brevet en Europe ", la Commission "estime que la création d'un brevet unique à l'échelle communautaire reste un objectif clé pour l'Europe ». Compte tenu des difficultés 
pour trouver un accord sur le brevet communautaire, d'autres accords juridiques ont été proposés en dehors du cadre légal de l'Union européenne afin de réduire les coûts de traduction (des brevets une fois délivrés) et des litiges, notamment l'Accord de Londres et l'Accord sur le règlement des litiges en matière de brevets européens (EPLA).

12. En 2007, 32 pays étaient parties au traité. De plus, l'OEB a conclu un «accord d'extension " avec cinq pays, qui offrent la possibilité d'étendre les brevets européens à ces pays sur demande, au moment de la demande de brevet européen.

13. Si le montant acquitté pour les désignations équivaut à au moins sept fois celui d'une désignation, alors tous les États parties à la Convention seront automatiquement considérés comme désignés, le demandeur ayant néanmoins la faculté de retirer ceux qu'il souhaite.

14. De ce fait, la part des enregistrements de demandes PCT entrant dans la phase II est plus élevée à l'OEB. Cela tient au caractère supranational de l'OEB, qui offre la possibilité de suivre une procédure unique pour plusieurs pays.

15. De plus, un demandeur a la faculté de prétendre à la priorité même si l'information dans la demande ultérieure n'est pas exactement la même que dans la demande antérieure, ou si plusieurs demandes "prioritaires " ont été combinées dans une seule et même demande étrangère. De ce fait, s'agissant des revendications de priorité, on peut s'attendre à ce que le nombre des demandes déposées dans les différents pays ne soit pas le même.

16. Dans le présent manuel, on utilise de façon interchangeable les expressions " demande PCT » et " demande internationale ».

17. Jusqu'en janvier 2004, le demandeur devait indiquer sur le formulaire de demande la liste précise des pays dans lesquels il pourrait souhaiter ultérieurement demander une protection. Cette obligation a ensuite été supprimée (mais les demandeurs peuvent donner une liste des pays dans lesquels ils n'ont pas l'intention de solliciter une protection, bien que cela ne modifie pas le montant des frais de la demande).

18. Le rapport préliminaire international sur la brevetabilité donne au demandeur des informations additionnelles sur la brevetabilité de son invention; ainsi, les demandeurs peuvent être mieux à même de décider s'il est ou non intéressant de poursuivre vers la phase nationale/régionale.

19. Toutefois, le droit national peut fixer des délais allant au-delà des 30 mois. Ainsi, il est possible d'entrer dans la phase régionale européenne 31 mois après la date de priorité. Les phases nationales et régionales peuvent également débuter plus tôt, à la demande expresse du demandeur [articles 20(3) ou 40(2)].

20. Dans le cas de demandes de continuation de brevets (par exemple CIP aux ÉtatsUnis), le décalage entre les priorités (première demande et demande dans d'autres pays) peut être plus important (en général, toutes les priorités renvoient à un an après la première priorité); cela aura donc un impact sur l'actualité des publications des brevets dans d'autres juridictions.

\section{Références}

Harhoff, D. et M. Reitzig (2002), « Determinants of Opposition Against EPO Patent Grants: The Case of Biotechnology and Pharmaceuticals ", CEPR Discussion Papers 3645.

Trilateral Statistical Report (2006), édition 2006. 

Chapitre 4

Critères de base pour la compilation d'indicateurs fondés sur les brevets 


\subsection{Introduction}

La compilation de statistiques sur les brevets implique certains choix méthodologiques. La difficulté pour les statisticiens est de choisir parmi plusieurs options les variables pertinentes pour la compilation des statistiques. Ces choix méthodologiques ont une influence significative sur les statistiques obtenues et leur interprétation. Les statistiques sur les brevets ne peuvent être valablement interprétées que si les critères et les méthodologies utilisées pour la compilation sont suffisamment connus.

La décision de choisir un critère plutôt qu'un autre est fonction des phénomènes à mesurer et des besoins des utilisateurs. Ainsi, il est possible d'élaborer différents indicateurs du nombre de demandes de brevets auprès du bureau des brevets du pays A pour rendre compte de l'activité d'invention dans d'autres pays, du pouvoir de marché d'entités originaires de différents pays dans le pays A (propriété de portefeuille de brevets) ou de l'attractivité du système de brevets du pays A, etc. S'il s'agit en particulier de mesurer l'activité inventive des pays, le critère de calcul de l'indicateur doit alors être le pays de résidence de l'inventeur, alors que si le but est de mesurer la propriété des inventions, le critère le plus approprié sera le pays de résidence du demandeur. De la même manière, si l'objectif est d'évaluer l'attractivité des pays dans le domaine de la protection, le ou les pays auprès desquels des demandes de brevets sont déposées constituent le critère le plus approprié.

Les choix méthodologiques de base les plus courants associés à la compilation de statistiques sur les brevets sont les suivants: date de référence, pays d'attribution et traitement de données agrégées comparables au plan international (PCT, familles). Des indicateurs plus fins fondés sur ces critères peuvent être envisagés par domaine technologique, région, institution d'origine, etc.

En règle générale, il est recommandé de ne pas réunir des indicateurs provenant de bureaux des brevets différents. Ainsi, le nombre de demandes de brevets déposées en Corée par des demandeurs coréens n'est pas comparable au nombre de brevets demandés en Australie par des demandeurs australiens (ou même coréens). Comme on le verra plus en détail, les procédures juridiques et administratives diffèrent selon les bureaux des brevets, et il existe un biais au niveau local dans le comportement des demandeurs (les demandeurs d'un pays ont tendance à demander davantage de brevets dans leur pays que les non-résidents). En conséquence, l'analyse présentée dans ce chapitre concerne les données provenant d'un seul et même bureau des brevets (ou les familles de brevets). 


\subsection{Date de référence}

Le problème du choix de l'année à laquelle un brevet est attribué est que le document brevet comprend plusieurs dates, qui reflètent l'historique de l'invention, la procédure d'établissement du brevet et de la stratégie du demandeur (Dernis et al., 2001; Hinze et Schmoch, 2004).

- La date de priorité (date du premier dépôt d'une demande de brevet, où que ce soit dans le monde, pour protéger une invention) est la date la plus ancienne et par conséquent peut être considéré comme la plus proche de la date de l'invention. Le chapitre 3 a montré que plusieurs voies peuvent être empruntées pour déposer une demande de brevet. La procédure de protection par brevet débute par une première demande; il s'agit de la demande initiale de brevet, avant toute demande ultérieure visant à étendre la protection à d'autres pays $^{1}$.

- La date de demande est la date à laquelle un brevet est demandé auprès d'un bureau des brevets particulier. Il existe un décalage de 12 mois entre résidents et étrangers pour les procédures directes traditionnelles, et celuici peut atteindre 30 mois pour les procédures PCT. En général, le demandeur déposera une demande auprès du bureau des brevets de son pays (ce qui crée la date de priorité) puis étendra sa demande ultérieurement à d'autres pays, soit en déposant une demande directement auprès des bureaux des brevets correspondants (ce qui génère une date de demande dont le décalage avec la date de priorité peut atteindre 12 mois) ou en déposant une demande de brevet selon la procédure PCT (le décalage étant de 12 mois pour le dépôt PCT luimême, mais il peut atteindre 30 mois pour le transfert vers la phase nationale) ${ }^{2}$.

- La date de publication (18 mois après la date de priorité sauf pour certaines demandes auprès de l'USPTO, publiées uniquement en cas de délivrance et au moment de celle-ci) indique le moment auquel l'information sur l'invention est divulguée auprès du grand public et mise à la disposition des statisticiens.

- La date de délivrance est la date à laquelle les droits de brevets sont conférés au demandeur par l'organisme autorisé. Il faut en moyenne trois ans à l'USPTO et cinq ans à l'OEB pour qu'un brevet soit délivré, mais la procédure peut prendre jusqu'à dix ans dans certains cas.

Pour rendre compte des performances en matière d'invention, les indicateurs fondés sur les dates de demande et/ou de délivrance présentent un certain nombre de biais associés à la procédure d'étude du brevet : les données sont tributaires de divers délais administratifs (notamment la procédure d'examen), du comportement stratégique du demandeur, et du fait que les données ne sont pas comparables entre pays dans la mesure où le décalage entre la date de priorité et les dates de demande (ou de délivrance) varie d'un pays à l'autre. Les inventeurs du pays A déposeront généralement leurs 
demandes auprès du bureau des brevets du pays $\mathrm{A}$ immédiatement après l'invention, alors que les étrangers ne feront leur demande qu'un an plus tard (année de priorité) : dans ces conditions, le fait de compter les deux types d'invention sur la base de l'année de la demande amène à comparer des inventions d'années différentes, ce qui peut introduire des biais en période d'évolution technologique rapide ou dans des pays où la technologie croît rapidement.

En ce qui concerne l'utilisation de la date de délivrance, cela signifie : i) que le comptage se limite à celui des délivrances (et exclut donc les demandes non satisfaites); ii) que l'information notifiée est déjà ancienne (il faut en moyenne trois à cinq ans pour délivrer un brevet); iii) que les inventions d'un grand nombre d'années différentes sont comptées ensemble. Dans tous les bureaux, il existe un délai de traitement et d'examen qui, dans certains cas, peut être très long. De ce fait, les statistiques fondées sur les brevets délivrés ne sont pas strictement comparables d'un bureau des brevets à l'autre, dans la mesure où le temps nécessaire pour délivrer un brevet varie entre les différents bureaux. De plus, comme les bureaux des brevets doivent faire face à une charge de travail considérablement augmentée depuis le milieu des années 90, la procédure a eu tendance à s'allonger, de sorte que le nombre des délivrances ne rendra compte de la dynamique sous-jacente que de façon lissée et décalée (effets de calendrier) ${ }^{3}$.

L'une des dates les plus significatives d'un point de vue technologique ou économique est la date de priorité. C'est la plus proche de la date de l'invention. D'autres détails découlent des contraintes légales (première priorité) ou des délais administratifs. On a pu montrer que les entreprises qui choisissent de breveter une invention le font très tôt, afin d'avoir la possibilité de retirer leur demande ultérieurement si l'invention se révèle décevante.

En conséquence, pour retracer les performances en matière d'invention, il est recommandé d'utiliser la date de priorité pour compiler les statistiques sur les brevets. Selon l'indicateur recherché - par exemple, activité de publication du bureau des brevets (date de publication) ou statut juridique des brevets (date de délivrance) - les autres critères sont également utiles. Ceux-ci cependant sont des indicateurs plus pauvres en informations sur la performance des pays.

Le tableau 4.A1.1 illustre la façon dont le choix de la date influe sur les indicateurs concernant les brevets. Le nombre total de brevets délivrés à l'OEB aux pays de l'OCDE en 2000 a été de 27139 si l'on prend la date de délivrance comme date de référence des brevets délivrés; mais ce chiffre passe à 31210 si l'on retient la date de priorité comme date de référence. De la même manière, pour les demandes de brevets, le nombre enregistré est de 146242 si l'on prend la date de priorité et de 134410 si on prend la date de la demande. Le décalage moyen des comptages entre date de priorité et date de demande 
(pour les demandes de brevets) était de $9 \%$ en 2000 pour l'ensemble des pays membres de l'OCDE. Pour les délivrances, l'écart était de $28 \%$. Les statistiques sur les brevets délivrés à l'OEB montrent l'impact du choix de la date sur les comparaisons entre pays. Pour les brevets délivrés, si l'on prend l'année 2000 comme date de priorité, c'est en Allemagne que l'on observe le plus fort taux de brevets parmi les pays de l'OCDE, devant les États-Unis. L'ordre s'inverse si l'on compte les brevets d'après la date de délivrance. Le taux le plus fort (26\%) est alors celui des États-Unis, devant l'Allemagne (20.6 \%) et le Japon (20.3\%). En ce qui concerne les demandes de brevets, les États-Unis affichent de loin la plus forte proportion (33.8 \% et $33.7 \%$, selon que l'on prend respectivement la date de priorité et la date de la demande).

\subsection{Pays de référence}

Le document brevet contient des informations sur le pays de l'inventeur, le pays du demandeur et le pays de priorité (pays dans lequel la première demande a été présentée). Tous ces éléments sont utiles, et il est intéressant de procéder à un examen comparatif de leur signification.

- Les comptages de brevets par pays de résidence du demandeur indiquent la «propriété " ou le contrôle de l'invention (c'est-à-dire le nombre de brevets détenus par les résidents de chaque pays). Ce type d'indicateur rend compte des performances innovantes des entreprises d'un pays donné, où que soient implantés leurs moyens de recherche.

- Les comptages de brevets selon le pays de résidence de l'inventeur indiquent l'inventivité des laboratoires locaux et de la main-d'œuvre d'un pays donné. L'adresse indiquée dans le document brevet est en général l'adresse professionnelle de l'inventeur (l'adresse du laboratoire où travaille l'inventeur).

- Les comptages de brevets par bureau de priorité (pays dans lequel la première demande a été présentée, avant que la protection ne soit étendue à d'autres pays) indiquent l'attrait du processus de protection par brevet dans le pays, la qualité de la réglementation en matière de propriété intellectuelle (règles et coûts de la protection par brevet), la réputation du bureau des brevets et les conditions économiques générales (par exemple, taille du marché). Ainsi, de nombreuses entreprises canadiennes déposent d'abord un brevet aux États-Unis, pour éventuellement l'étendre ensuite au Canada.

Il est recommandé d'utiliser le pays de résidence de l'inventeur pour compiler des statistiques sur les brevets rendant compte de l'activité d'invention. Le pays de résidence du demandeur est utile pour analyser les stratégies de répartition des marchés des entreprises, notamment des multinationales. 
Une difficulté courante lors de la compilation d'indicateurs par pays de résidence du demandeur est que le brevet peut être demandé par une filiale de l'entité ayant le contrôle de l'invention. Certaines grandes entreprises multinationales ont des filiales spécialisées dans l'enregistrement des brevets, qui peuvent même être implantées dans un pays qui n'est pas celui de la société-mère, ce qui « bruite » les données. L'implantation de ces demandeurs affiliés peut également être guidée par des considérations fiscales ou autres. Dans ce cas, il est préférable d'attribuer le brevet à l'entité de contrôle (sociétémère), ce qui peut nécessiter le rapprochement des données sur le brevet avec des informations sur la propriété provenant d'autres sources.

Le tableau 4.A1.2 illustre l'impact de ces critères sur les statistiques concernant les brevets. Il indique les parts des pays de l'OCDE dans les demandes auprès de l'OEB sur la base de différents critères de comptage pour la répartition géographique. Des petits pays comme la Belgique, la Hongrie, le Mexique et la République tchèque affichent des parts élevées comme pays d'inventeurs (l'écart par rapport au total des inventions variant entre 15 et $27 \%$ ). Inversement, les Pays-Bas, la Suisse ou la Finlande enregistrent davantage de brevets comme pays demandeurs que comme pays inventeurs. Cela s'explique par l'internationalisation plus poussée des activités de recherche (propriété nationale d'inventions réalisées à l'étranger). Une exception notable est le Luxembourg, dont la part dans le total de l'OCDE comme pays demandeur est le double de sa part comme pays inventeur.

Brevets ayant plusieurs inventeurs de différents pays. Au cours des années récentes, on a pu noter une intensification de la coopération entre chercheurs de pays différents, ce qui traduit l'ouverture et l'internationalisation des activités scientifiques et technologiques. Cette information se retrouve dans les documents de brevet répertoriant plusieurs inventeurs provenant de pays différents. Ces brevets peuvent soit être attribués partiellement à chaque pays mentionné (comptage fractionnaire) soit être intégralement attribués à chaque pays cité. Le comptage fractionnaire peut être utilisé quand plusieurs inventeurs (ou demandeurs, ou classes IPC) sont mentionnés dans les données du brevet de manière à créditer au prorata chaque unité étudiée, et éviter ainsi les doubles comptages.

Le comptage fractionnaire est particulièrement indiqué pour compiler des statistiques sur les brevets lors du calcul de totaux régionaux ou mondiaux car il réduira le biais introduit par les doubles comptages, mais le comptage simple est parfois préférable selon la question à examiner (par exemple pour mesurer l'internationalisation des activités technologiques par pays) ${ }^{4}$. 


\subsection{Demandes PCT}

\subsubsection{Comptage des demandes PCT dans la phase internationale}

Les indicateurs de brevets construits au moyen d'informations provenant d'un seul bureau des brevets présentent certains points faibles. Le biais lié au pays d'origine en est un, dans la mesure où en proportion de leur activité d'invention, les demandeurs ressortissants d'un pays ont tendance à déposer davantage de brevets dans leur pays que les non-résidents. La part relative des demandeurs étrangers est affectée par des facteurs qui n'ont pas un lien direct avec la technologie, comme la densité et l'orientation des liens commerciaux entre chacun de ces pays et le pays dans lequel les brevets sont déposés. Des exportations plus fortes ou un investissement direct plus élevé du pays A vers le pays $\mathrm{B}$ conduiront à une augmentation du nombre de brevets émanant de demandeurs du pays $\mathrm{A}$ dans le pays $\mathrm{B}$, qui sont destinés à protéger leurs technologies. Il existe deux types d'indicateurs sur les brevets qui sont relativement libres de ce type de biais et sont donc mieux adaptés que les demandes nationales ou régionales pour les comparaisons entre pays : ce sont les demandes PCT et les familles de brevets (ces dernières sont examinées dans la section 4.5).

La procédure en relation avec le Traité de coopération en matière de brevets (PCT) a une vocation internationale, et elle est administrée par l'Organisation mondiale de la propriété intellectuelle. Chaque demande présentée via le PCT désigne l'ensemble des États signataires du traité - et ce depuis 2004; auparavant, une liste des États désignés devait être fournie par le demandeur et les taxes à acquitter variaient selon le nombre d'États désignés. Dans ce contexte, une demande PCT peut être considérée comme une demande mondiale de brevets qui présente beaucoup moins de biais vis-à-vis de l'ensemble des pays que les demandes nationales. Un autre avantage du PCT est qu'il est de plus en plus utilisé par des demandeurs de tous les pays membres. Le graphique 4.2 montre l'augmentation soutenue du nombre de dépôts via le PCT (désignant l'OEB). Cette multiplication des demandes fait du PCT une source de plus en plus pertinente pour l'établissement de statistiques. Depuis le début des années 2000, la plupart des pays sont bien représentés, notamment le Japon et la Corée (qui sont venus tardivement à l'utilisation de la procédure PCT). De plus, le PCT rend compte assez bien des activités technologiques de pays émergents (Brésil, Russie, Chine, Inde, etc.). Il faut tenir compte du fait que le PCT a commencé à être utilisé plus largement après 1990, de sorte qu'il existe une période de transition jusqu'à environ 2000 dans laquelle les comparaisons entre pays ou dans le temps doivent être interprétées avec prudence.

Les informations PCT présentent deux inconvénients : premièrement, elles ne sont pas entièrement exemptes de biais, dans la mesure où les demandeurs utilisent cette procédure de façon inégale selon les pays, du fait de contraintes 


\section{Graphique 4.1. Part des pays dans les brevets déposés dans les trois grandes régions, 2005}
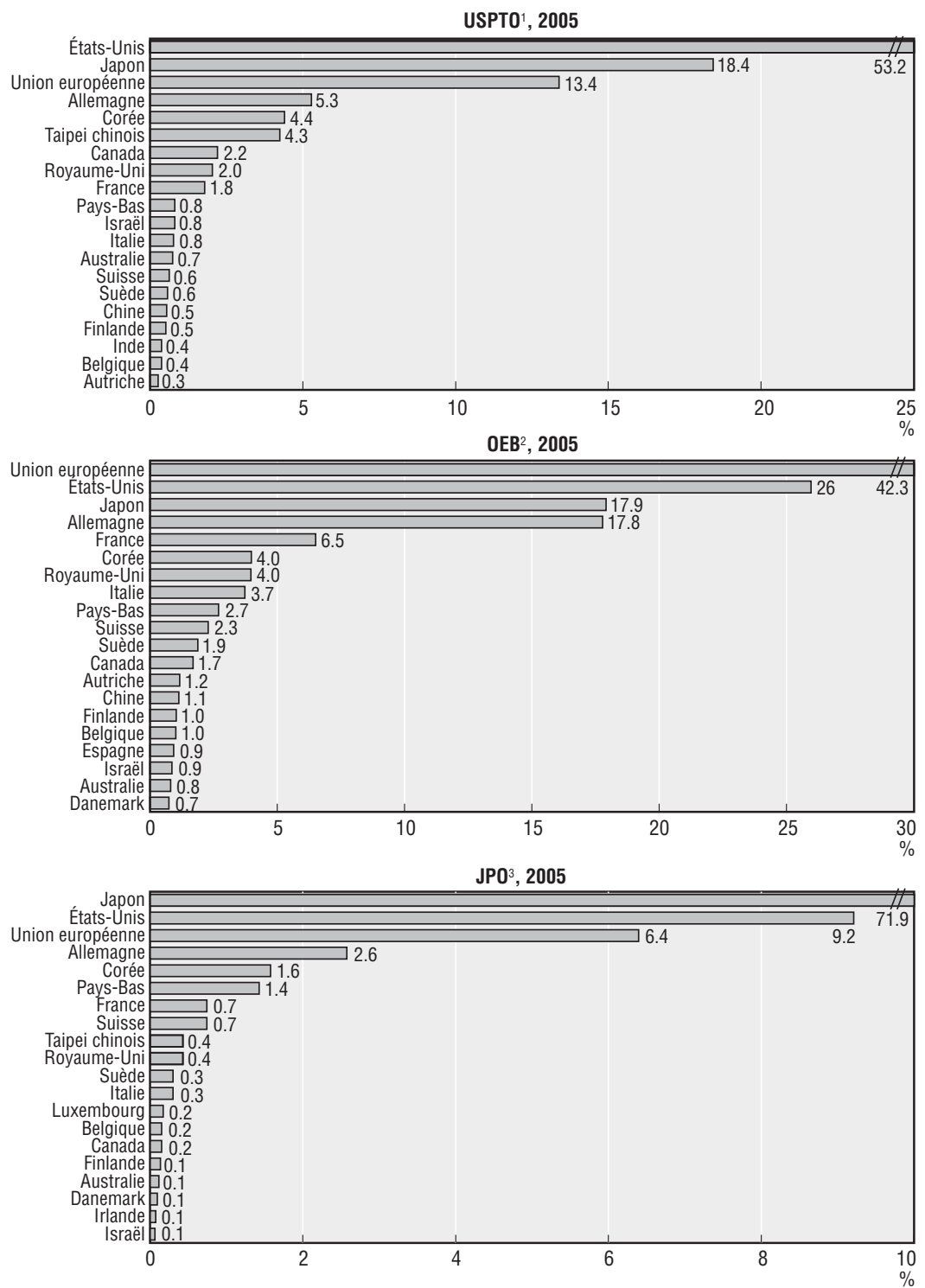

1. Demandes de brevets auprès de l'USPTO. Les comptages de brevets sont basés sur le pays de résidence du premier inventeur nommé et sur la date de la demande.

2. Demandes de brevets auprès de l'OEB, englobant les demandes Euro-direct et Euro-PCT dans la phase régionale. Les comptages de brevets sont basés sur la date de priorité, le pays de résidence de l'inventeur et le comptage fractionnaire. Les chiffres pour 2004 et 2005 sont des estimations.

3. Demandes de brevets auprès du JPO. Les comptages de brevets sont basés sur le pays de résidence du demandeur et la date de demande, ainsi que le comptage fractionnaire. Les chiffres pour 2001 à 2005 sont des estimations fondées sur les rapports annuels du JPO.

Sources : Rapports sur les statistiques de brevets de l'USPTO; OCDE, base de données sur les brevets, juin 2007; base de données sur les brevets de l’IIP, 2005 et rapports annuels du JPO. 


\section{Graphique 4.2. Brevets demandés selon la procédure $\mathbf{P C T}^{1}$, désignations de l'OEB}

Nombre total, taux de croissance et grandes régions

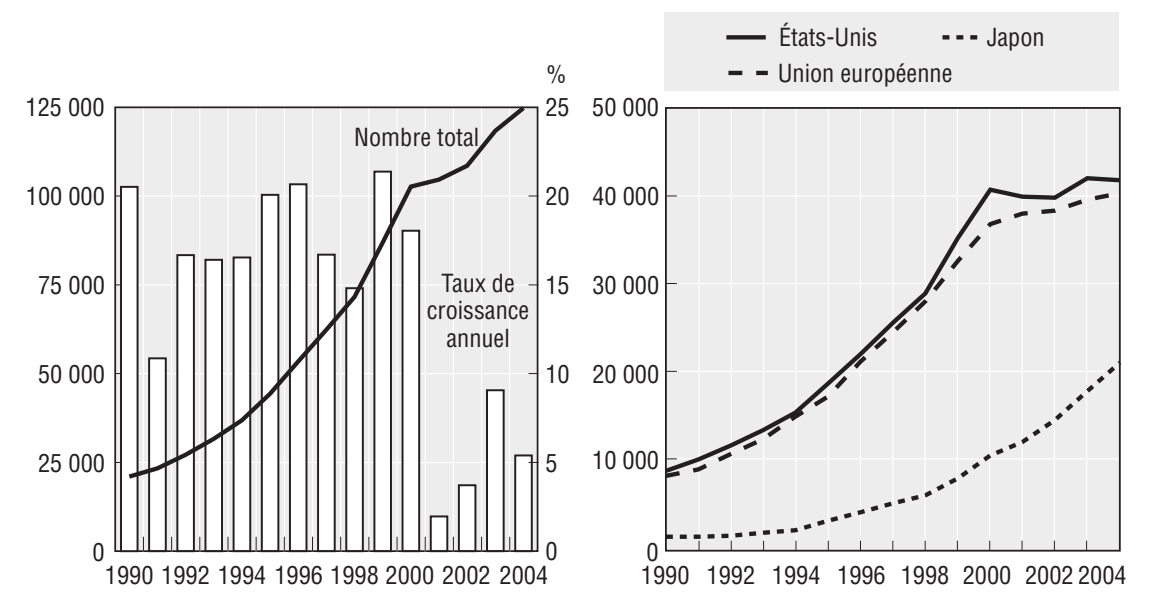

Note: Les comptages de brevets sont basés sur la date de priorité, le pays de résidence de l'inventeur et le comptage fractionnaire.

1. Demandes de brevet selon la procédure PCT, dans la phase internationale, désignant l'OEB.

Source: OCDE, base de données sur les brevets.

juridiques ou pour des raisons économiques. Deuxièmement, les demandes PCT ne sont pas des demandes de brevets au même sens que les demandes nationales. Il s'agit plutôt d'options pour de futures demandes auprès des bureaux des brevets du monde entier, puisque la procédure PCT comprend deux phases: une phase internationale, éventuellement suivie d'une phase nationale/régionale (voir le chapitre 2 pour plus de précisions). Le coût relativement faible d'une demande de brevet au plan international fait que la procédure PCT est peu sélective - en cas d'incertitude quant à la valeur de leurs inventions, les demandeurs peuvent présenter une demande " au cas où " et remettre à plus tard la décision, plus coûteuse, d'une véritable demande. En conséquence, de nombreuses demandes PCT couvriront des inventions dont la valeur, ultérieurement, se révélera faible. De fait, une proportion non négligeable de demandes PCT n'évolue jamais vers la phase nationale. Cela amplifie l'inconvénient des comptages de brevets qui traitent de façon égale des inventions de valeur très inégale. Il faut également noter que même si les coûts sont plus faibles qu'une demande en parallèle dans de nombreux pays, les coûts de la demande par l'intermédiaire du PCT restent significatifs et supérieurs à ceux des demandes nationales.

\subsubsection{Comptage des demandes PCT dans la phase nationale}

La procédure en deux phases du PCT a d'importantes implications pour la compilation de statistiques sur les brevets. Faut-il rendre compte des données 
dans la phase internationale, qui est l'unique option pour les demandes futures, en parallèle avec les autres demandes nationales standard ou faut-il ne considérer que les demandes PCT entrant dans la phase nationale/ régionale, dans laquelle la décision est prise d'accorder le brevet ou de le rejeter? De la même manière, le système de désignation de la demande PCT a des implications pour la notification des statistiques sur les brevets. Faut-il compter tous les pays désignés dans la compilation des demandes PCT au niveau national ou bien doit-on ne considérer que les pays désignés dans lesquels la demande PCT se poursuit dans la phase nationale/régionale?

Pour certains pays, le fait de prendre en compte les données correspondant à la phase internationale modifierait fortement la part des brevets nationaux (voir les graphiques 4.3 et 4.4 ). Ainsi, pour un pays comptant 10000 demandes nationales par an (dans une grande majorité de pays, le chiffre est moindre), la prise en compte des demandes PCT (plus de 100000 demandes par an) diluerait énormément la signification des statistiques nationales, d'autant que la plupart de ces demandes PCT ne seront pas transférées au pays et ne déboucheront donc jamais sur de véritables demandes nationales. Les statistiques disponibles montrent qu'une forte proportion des demandes PCT initiales ne débouchent pas sur la phase nationale/régionale (OCDE, 2005).

Cependant, un inconvénient majeur si l'on inclut uniquement les demandes PCT entrant dans la phase nationale ou régionale de l'OEB est que cela aura un effet négatif sur l'actualité des indicateurs sur les brevets. Le délai peut atteindre 31 mois entre la date de priorité (c'est-à-dire la date de premier dépôt d'une demande de brevet où que ce soit dans le monde) et le moment où les demandes PCT entrent dans la phase nationale ou régionale. En conséquence, aucune statistique sur les brevets fondée sur cette approche sélective ne pourrait être produite avant 31 mois suivant la date considérée ${ }^{5}$.

\subsubsection{Rétropolation des demandes de brevets}

Une solution au problème d'actualité des données sur les demandes PCT est d'estimer (par rétropolation) le nombre de demandes PCT qui sera transféré à un pays donné. Il existe différents moyens de le faire (voir encadré 4.1). Pour rétropoler les demandes Euro-PCT, un moyen est de prévoir les demandes à partir des taux de transfert des demandes de brevets en vertu du PCT dans la phase régionale de l'OEB, étant donné que l'information sur les brevets PCT dans la phase internationale est révélée avant l'entrée dans la phase régionale/nationale (Schmoch, 1999). Une procédure de rétropolation en deux étapes peut être mise en œuvre sur la base du taux de transfert (voir encadré 4.2 ; Dernis, 2007).

Les bureaux des brevets prévoient les demandes de brevets afin de planifier la demande future de services. Un certain nombre de méthodologies de régression et d'enquête peuvent être adoptées (voir encadré 4.1). Les 


\section{Graphique 4.3. Part des pays dans les brevets demandés selon la procédure PCT ${ }^{1}, 2004$}

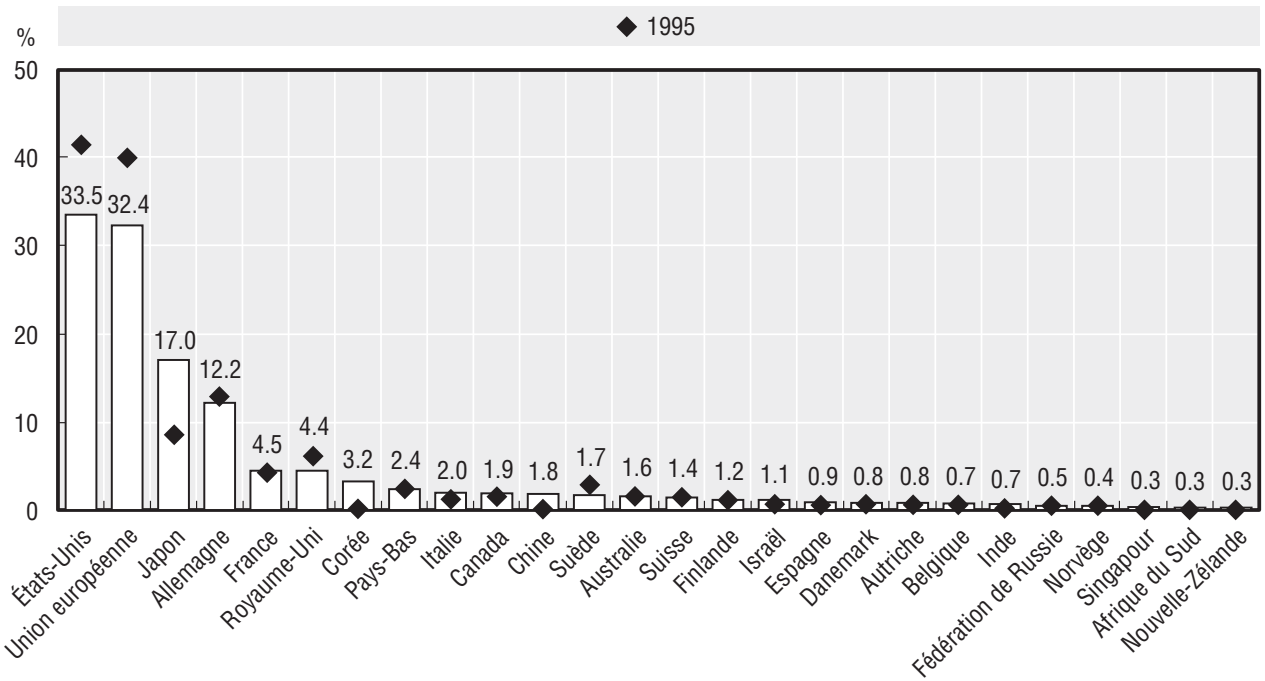

Graphique 4.4. Part des demandes Euro-PCT entrant dans la phase régionale ${ }^{2}, 2002-04$

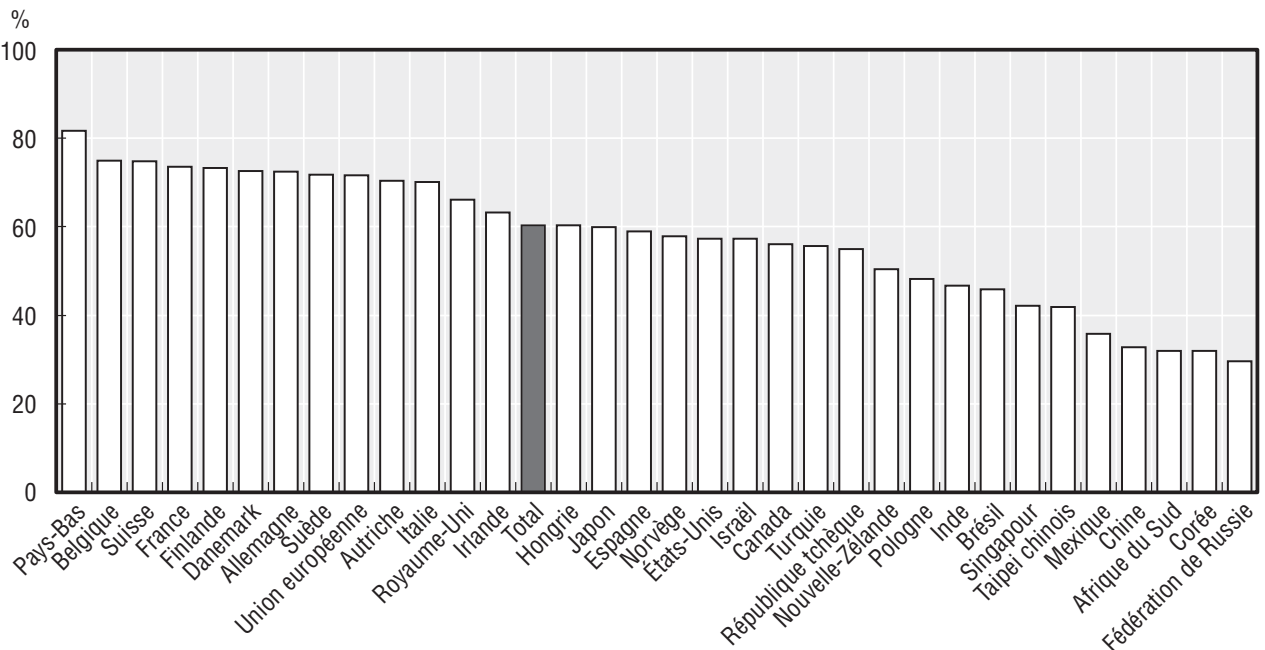

Note : Les comptages de brevets sont basés sur la date de priorité, le pays de résidence de l'inventeur et le comptage fractionnaire.

1. Demandes de brevet déposées selon le PCT, dans la phase internationale, désignant l'OEB.

2. Le graphique ne couvre que les pays dans lesquels au moins 250 brevets ont été déposés en vertu du PCT pour la période 2002-04.

Source: OCDE, base de données sur les brevets. 


\section{Encadré 4.1. Méthodologies de rétropolation}

Différentes méthodes ont été élaborées pour la rétropolation des brevets. Chaque donnée concernant les brevets (par exemple, USPTO, OEB, etc.) présente ses spécificités, il n'y a pas de modèle unique adapté à la structure intrinsèque des données, surtout en ce qui concerne les tendances : stationnaire, linéaire, exponentielle, etc. Diverses études ont déjà abordé les questions de rétropolation et de prévision ou expérimenté différentes méthodologies pour différents ensembles de données (OEB, PCT, par pays, par branche, etc.). Parmi ces études, au moins trois types de procédures d'estimation ont été utilisés :

- L'analyse de tendances consiste en une simple extrapolation des tendances sur diverses périodes de temps : modèles à moyenne mobile intégrée autorégressive - ARIMA (Dehon et van Pottelsberghe, 2003).

- Les modèles de transferts prédisent les transferts de premières demandes (prioritaires) au bureau des brevets - cela nécessite une bonne évaluation des premières demandes (qui sont partiellement disponibles car l'information n'a pas encore été rendue publique); transfert des demandes PCT vers la phase régionale (Schmoch, 1999; Dernis, 2007).

- Les modèles économétriques prédisent les demandes de brevets sur la base de modèles empiriques (fonctions de production de connaissances, Hausman et al., 1981) au moyen d'indicateurs économiques comme les dépenses de R-D par secteurs et sources de financement; le PIB; le nombre de chercheurs; la valeur ajoutée; les indicateurs d'opportunités technologiques (évolutions spécifiques dans certaines technologies); les indicateurs basés sur des informations spécifiques émanant des bureaux des brevets (budget, nombre d'examinateurs de brevets, taxes sur les brevets, etc.); les modèles probabilistes, etc. (Dehon et van Pottelsberghe, 2003).

méthodes par régression ont le désavantage que les prévisions reposent uniquement sur des données historiques et donc supposent la poursuite des tendances établies. Les modèles de régression linéaire peuvent être ajustés aux totaux des demandes annuelles, bien qu'une extension plus utile consiste à utiliser les tendances des premières demandes à l'échelle mondiale, puis de suivre leurs transferts en pourcentage à l'étranger vers d'autres bureaux dans un délai d'un an selon les principes de la Convention de Paris pour les demandes faisant état d'une priorité antérieure. Il existe également des possibilités intéressantes de modélisation des demandes mondiales de brevets simultanément auprès des différents bureaux en analysant les demandes prioritaires internationales de familles de brevets. On peut aussi utiliser des approches économétriques, qui en général impliquent l'utilisation de variables prédictives telles que le produit intérieur brut (PIB) ou les dépenses de recherchedéveloppement (R-D) ou les comptages de personnel de R-D dans les principaux 


\section{Encadré 4.2. Méthodes de rétropolation basées sur les transferts}

Un moyen de rétropoler les demandes de brevets est de prévoir les demandes futures en se basant sur les transferts des années précédentes. Ainsi, une procédure en deux étapes de rétropolation des demandes OEB consiste tout d'abord à estimer le nombre de demandes Euro-PCT qui sont entrées dans la phase régionale de l'OEB dans l'année $t-2$ (Schmoch, 1999; Dernis, 2007). Puis, les estimations des demandes Euro-PCT dans la phase régionale sont ajoutées au nombre de demandes OEB directes pour obtenir une estimation des demandes OEB totales dont la date de priorité se situe dans l'année $t-2$. La deuxième étape consistera à évaluer le nombre de demandes Euro-Directes et Euro-PCT dans la phase internationale pour la date de priorité $t-1$, au moyen de données $t-1$ partielles, avant de procéder de nouveau à la méthode de rétropolation indiquée dans l'étape 1 . Il est possible de procéder par simple méthode arithmétique, en utilisant les taux de transfert Euro-PCT de l'année $t-1$ ou la moyenne $\{t-1, t-2\}$ comme estimation des taux de transfert Euro-PCT (EPCT_TR) dans l'année $t$ :

$$
E P C T_{-} T_{1}=\frac{E P C_{t-1}}{P C T_{t-1}} \text { ou EPCT_TR } 1=\frac{\left(E P C T_{t-1}+E P C T_{t-2}\right)}{\left(P C T_{t-1}+P C T_{t-2}\right)}
$$

dans laquelle EPCTt désigne les demandes Euro-PCT dans la phase régionale à l'année $t$; et PCTt le nombre de demandes PCT désignant l'OEB à l'année $t$. Il est possible par des modèles linéaires simples d'estimer le taux de transfert des demandes Euro-PCT dans l'année $t$ en fonction soit du taux de transfert Euro-PCT à l'année $t-1$ soit du taux de transfert moyen des deux années antérieures. Des variables supplémentaires peuvent être ajoutées au modèle, par exemple la progression des demandes PCT entre t et $t-1$. Ces méthodes donnent des estimations robustes jusqu'à l'année $t-2$, même si l'activité de dépôts de brevets des pays peu actifs dans ce domaine ou des économies émergentes est difficile à prévoir, tant en niveau qu'en évolution (Dernis, 2007). On a pu constater que les tendances en matière de dépôts de brevets étaient plus irrégulières pour les pays où l'on dépose moins de brevets et pour certains économies/pays émergents (Khan et Dernis, 2005). Il est donc recommandé d'être prudent dans l'application de ces méthodes à ces pays.

pays sources de demandes. Les modèles de prévision incluent fréquemment une structure à correction d'erreurs de type ARIMA sur les diverses séries d'entrées et de sorties. La prévision à court terme de la demande à partir des comptages mensuels des demandes peut également être utile pour une planification plus détaillée, et les bureaux des brevets ont aussi bien entendu besoin d'établir des prévisions de leur charge de travail pour les différentes phases de leurs procédures d'examen. 
Les enquêtes auprès des demandeurs présentent l'avantage qu'il est possible d'observer relativement rapidement les changements d'opinion sur les pratiques en matière de demandes de brevets. L'OEB et le JPO mène des enquêtes de leurs clients tous les ans ou tous les deux ans. En général, il est demandé aux personnes interrogées d'indiquer le nombre effectif et prévu de leurs demandes de brevets sur l'année antérieure et jusqu'aux trois années à venir. Il est possible de regrouper les estimations des taux de croissance ainsi obtenues et d'en faire la moyenne de diverses façons pour obtenir des prévisions quantitatives à court terme des demandes futures de brevets. Bien que cette méthode permette aux bureaux de réagir rapidement aux évolutions des tendances, les prévisions à base d'enquêtes elles-mêmes peuvent ne pas être aussi efficaces que les méthodes par régression en situation normale, car la régression est une méthode qui institutionnalise les tendances lourdes. Les enquêtes ont aussi l'avantage de permettre de recueillir parallèlement des informations microéconomiques sur les demandeurs, qui peuvent être utiles d'autres façons pour aider les bureaux des brevets à en savoir plus sur les besoins et les spécificités de leurs clients.

\subsection{Familles de brevets}

Les familles de brevets sont un autre moyen d'établir des indicateurs sur les brevets qui soient comparables entre pays. L'ensemble des brevets (ou demandes) déposés dans plusieurs pays qui ont un lien les uns avec les autres du fait d'une ou plusieurs demandes prioritaires communes sont généralement considérés comme constituant une famille de brevets. On considère souvent aussi qu'une famille de brevets regroupe tous les brevets protégeant la même invention, bien que selon la définition de la famille et l'étendue des liens entre les membres de la famille, cela puisse être plus ou moins vrai. Des différences dans les systèmes et procédures nationales en matière de brevets peuvent conduire à des variations dans l'étendue de la protection demandée et accordée dans les premières demandes et les demandes ultérieures. On trouvera dans cette section certaines définitions couramment utilisées pour les familles de brevets, en notant qu'il s'agit d'un domaine dans lequel les recherches se poursuivent et où de nouvelles définitions sont étudiées par les chercheurs et praticiens pour mieux rendre compte des stratégies des demandeurs.

L'étendue et la composition d'une famille de brevets sont fonction de la nature des liens de priorité, de la nature du document brevet et des bureaux des brevets pris en compte dans sa définition. Un type particulier de familles est celui des familles de brevets triadiques (Grupp et al., 1996). Selon la définition de l'OCDE (Dernis et al., 2001), une famille de brevets triadique est un ensemble de demandes de brevets déposées auprès de l'OEB et du JPO, et de brevets délivrés par l'USPTO qui partagent une ou plusieurs demandes prioritaires. La restriction aux brevets délivrés par l'USPTO (plutôt que les demandes) tient au 
fait que jusqu'en 2001, les demandes n'étaient pas publiées par l'USPTO, ce qui rendait impossible ce type de statistiques fondées sur les demandes. Un autre type de famille est celui utilisé dans le Trilateral Statistical Report, qui dénombre toutes les priorités demandées, chacune étant considérée comme une famille. Cette méthode est utile pour rassembler des statistiques sur les flux entre le lieu de première demande et les activités dans d'autres bureaux sur la base des priorités de la Convention de Paris.

Pour l'analyse statistique, les familles de brevets triadiques améliorent la comparabilité internationale des indicateurs fondés sur les brevets, car seuls les brevets demandés dans le même ensemble de pays sont inclus dans la famille; les biais liés au pays d'origine et à l'influence de l'implantation géographique sont donc éliminés. Deuxièmement, les brevets inclus dans la famille sont en général de forte valeur; les détenteurs du brevet n'engagent les coûts additionnels et n'acceptent les délais de l'extension de la protection à d'autres pays que s'ils les jugent intéressants. En introduisant de fait un point de bascule sur la valeur des brevets inclus dans cet ensemble, on choisit sur la courbe de distribution des brevets la partie correspondant aux plus fortes valeurs (s'agissant des demandes mondiales), ce qui fait que les comptages des familles de brevets apportent davantage d'informations que les comptages nationaux ou régionaux.

Pour le comptage des familles de brevets triadiques destiné à rendre compte des performances en matière d'invention, il est recommandé d'utiliser la date de priorité la plus ancienne (première demande du brevet à l'échelle mondiale), le pays de résidence de l'inventeur et les comptages fractionnaires (voir le graphique 4.5).

Une définition de la famille de brevets assez restrictive est celle des brevets équivalents, qui considère uniquement les documents ayant exactement les mêmes priorités. Tel est le cas quand un demandeur présente d'abord une demande unique dans son pays d'origine (demande prioritaire unique) puis dans l'année qui suit sollicite une protection dans d'autres pays. Selon les règles de la Convention de Paris, il a le droit de se référer à la priorité de la demande qu'il a faite dans son pays d'origine, de sorte que toutes ses demandes ultérieures équivaudront à sa demande prioritaire. Les brevets équivalents sont généralement considérés comme les membres des familles de brevets ayant entre eux les liens les plus étroits, et sont donc les plus susceptibles de protéger la même invention.

Un inconvénient des familles triadiques de l'OCDE est que les données les concernant ne sont pas d'une extrême actualité. Pour l'USPTO, le délai moyen entre la demande et la délivrance est d'environ 35 mois, mais il peut atteindre 44 mois. En conséquence, aucune statistique complète des familles triadiques n'est disponible avant au moins trois ans environ après la date considérée. Il est possible de remédier à cet inconvénient par « rétropolation » des familles 


\section{Graphique 4.5. Part des pays dans le total des familles de brevets triadiques ${ }^{1}, 2005$}

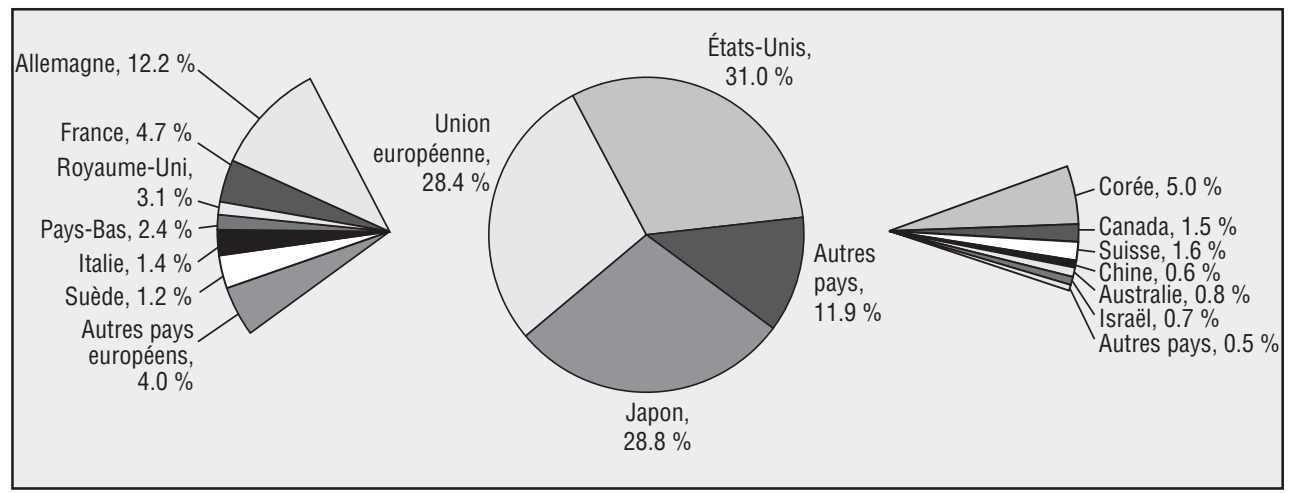

Notes : Les comptages de brevets reposent sur la date de priorité la plus ancienne, le pays de résidence de l'inventeur et le comptage fractionnaire. Les données sont tirées pour l'essentiel de la base statistique mondiale sur les brevets de l'OEB (avril 2007).

1. Ensemble des brevets demandés à l'OEB, l'USPTO et le JPO. Les chiffres à partir de 1998 sont des estimations. Source: OCDE, base de données sur les brevets.

de brevets (voir la section 4.4.3), c'est-à-dire en utilisant les informations disponibles du passé pour estimer les nombres les plus vraisemblables de familles à l'avenir (Dernis, 2007). Comme on l'a vu, il est possible d'utiliser une méthode en deux temps, en étendant la période couverte par les familles de brevets triadiques jusqu'à l'année $t-3$, et éventuellement l'année $t-2$.

$\mathrm{Au}$ moment de compiler des indicateurs internationaux, un choix doit être fait entre les demandes PCT et les familles de brevets. Celui-ci sera fonction de l'actualité et de la qualité recherchée pour les indicateurs. Les demandes PCT présentent un avantage en termes d'actualité (elles sont publiées 18 mois après la date de priorité) tandis que les familles de brevets

\section{Graphique 4.6. Exemple des familles de brevets au sens étroit et au sens large}

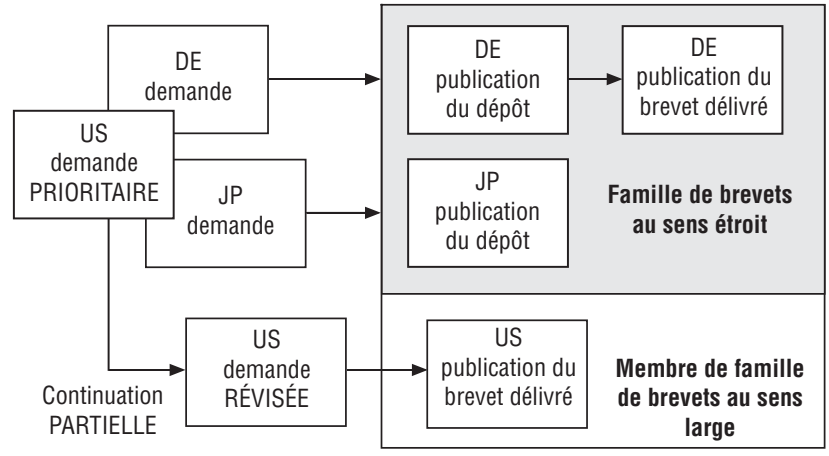




\section{Graphique 4.7. Familles de brevets triadiques rapportées au PIB ${ }^{2}, 2005$}

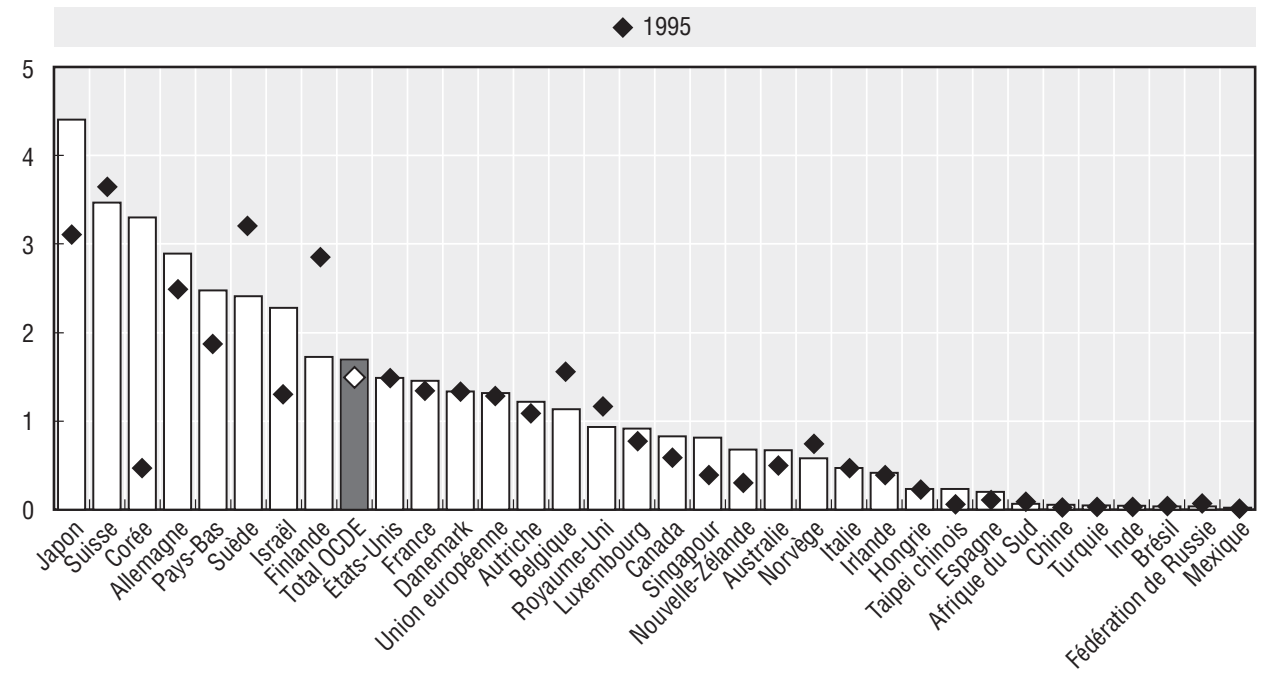

Graphique 4.8. Familles de brevets triadiques ${ }^{1}$ par millions d'habitants, 2005

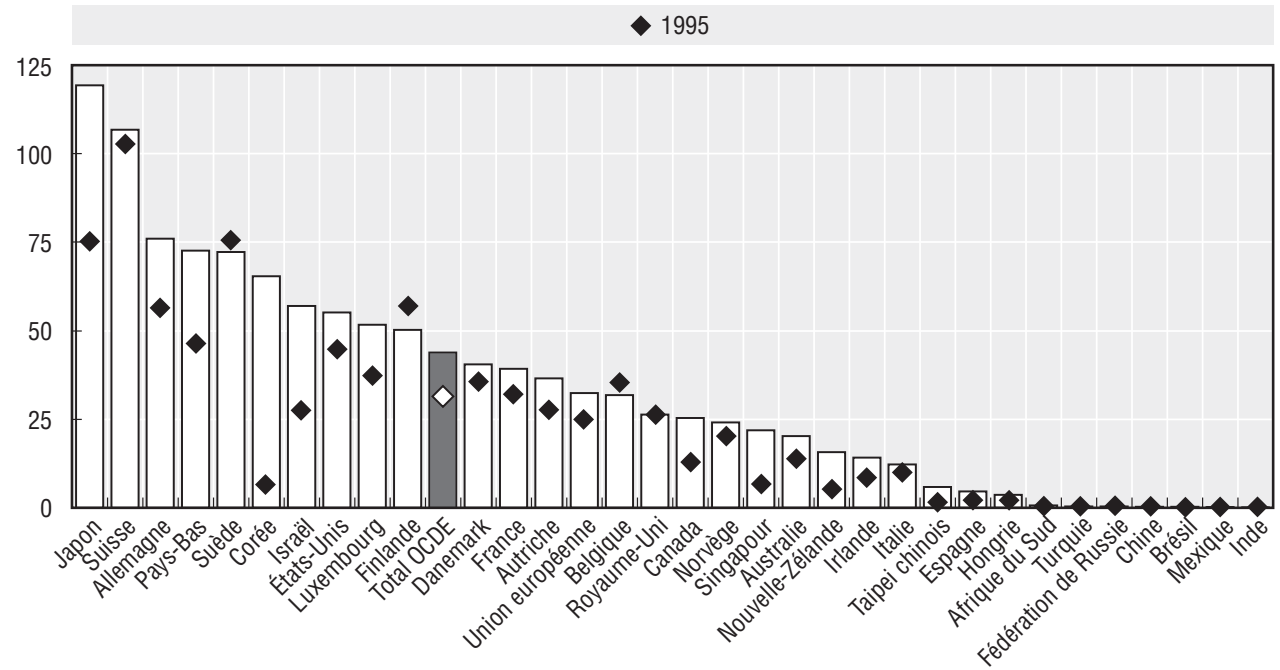

Note: Les comptages de brevets reposent sur la date de priorité la plus ancienne, le pays de résidence de l'inventeur et le comptage fractionnaire. Les données sont tirées pour l'essentiel de la base statistique mondiale sur les brevets de l'OEB.

1. Ensemble des brevets demandés à l'OEB, l'USPTO et le JPO. Les chiffres pour 2005 sont des estimations. Seuls les pays/économies avec plus de 20 familles en 2005 sont inclus.

2. Produit intérieur brut (PIB), milliards de USD (2000) en parités de pouvoir d'achat.

Source: OCDE, base de données sur les brevets. 
présentent un avantage en termes de qualité (on sélectionne les inventions de forte valeur visant à couvrir les principaux marchés internationaux).

Il existe aussi d'autres définitions des familles de brevets (voir encadré 4.A1.1 en annexe 4.A1). On choisira le concept de famille selon la nature de l'analyse à entreprendre. Si l'on veut prendre en compte, par exemple, les petites inventions ayant un marché essentiellement local, la définition englobant « toutes les priorités " sera préférée aux familles triadiques, car ces dernières éliminent sciemment ces petites inventions. Si l'on souhaite en revanche compiler les inventions à forte valeur, comparables entre pays, les familles triadiques seront préférées.

On peut envisager aussi des définitions des familles de brevets plus larges. Elles sont en général constituées à partir de relations complexes, impliquant plusieurs demandes prioritaires communes - ou en tout cas au moins une - dans différents pays; ou à partir de relations résultant de divisions, de continuations ou de continuations partielles s'agissant de l'USPTO (voir le graphique 4.6 pour un exemple) :

- Demande divisionnaire : c'est le cas lorsque le demandeur divise la demande initiale en plusieurs demandes divisionnaires, chacune revendiquant une invention différente incluse dans la demande initiale.

- Continuations : c'est le cas lorsqu'une deuxième demande ou une demande ultérieure est déposée alors que la demande d'origine est en cours. À l'USPTO, une continuation partielle (continuation-in-part - CIP) désigne une deuxième demande ou une demande ultérieure, incluant de nouveaux éléments protégés, alors que la demande d'origine est en cours.

\subsection{Indicateurs fondés sur les brevets normalisés au niveau des pays}

Le niveau de brevetage d'un pays dépend de facteurs institutionnels, de la nature du système juridique et de divers facteurs liés aux dimensions du pays : la taille de sa population, de son économie (PIB), de sa R-D et de sa communauté de chercheurs. Le comptage des brevets peut être normalisé au moyen de ces variables démographiques, économiques et de R-D pour obtenir des indicateurs qui ne tiennent pas compte de la taille et fournissent des informations non biaisées permettant de comparer le niveau de brevetage des pays. En comparaison internationale, le classement des pays change de manière significative lorsque les indicateurs sont normalisés.

Deux indicateurs fréquemment utilisés par l'OCDE comme indicateur de l'intensité de brevetage d'un pays sont les brevets triadiques par rapport au PIB et par habitant (graphiques 4.7 et 4.8). Un autre indicateur fréquemment utilisé est le nombre de brevets d'un pays par rapport à la R-D financée par l'industrie. Cet indicateur montre la productivité des investissements par les 
entreprises en R-D. Il peut tenir compte d'un décalage entre la performance de la R-D et les demandes de brevets correspondants. Toutefois, Hall et al. (1986) ont trouvé une relation temporelle assez étroite entre la R-D et les demandes de brevets au niveau de l'entreprise. Lorsque les données de R-D sont disponibles, de tels indicateurs peuvent également être calculés au niveau de l'entreprise, de l'institution ou de la région.

\section{Notes}

1. Aux États-Unis, la date de conception entre en jeu en cas d'interférences (la règle du " premier à inventer").

2. S'agissant du PCT, il faut noter qu'après le transfert vers la phase nationale ou régionale, il faut environ six mois de plus pour que cette phase soit publiée par le bureau régional/national. Dans le cas d'une demande Euro-PCT, l'information sur le transfert effectif auprès de l'OEB est disponible 36 mois après la date de priorité (première demande).

3. La notification des données par année de délivrance est particulièrement courante pour les brevets de l'USPTO pour la raison que, jusqu'en 2002, l'USPTO ne publiait que les brevets délivrés, et non les demandes. Toutefois, même lorsque tel est le cas, l'année de délivrance produit des informations biaisées sur les inventions.

4. Ainsi, si le thème étudié est l'inventivité d'un pays isolé (ou d'une région ou d'une branche), le comptage fractionnaire fondé sur le pays de résidence des inventeurs peut ne pas être pertinent, et il faudrait préférer le comptage simple. L'utilisation du comptage fractionnaire est commode pour le calcul de valeurs agrégées mais elle peut être discutable car elle soulève la question de savoir par exemple dans quelle mesure une fraction d'un brevet comptant plusieurs inventeurs peut présenter moins de valeur pour une unité d'analyse donnée (pays ou région, etc.) qu'un brevet ayant un inventeur unique.

5. Certaines procédures des bureaux des brevets pendant la phase internationale peuvent influer sur le choix du demandeur de poursuivre ou non dans la phase nationale/régionale, notamment le rapport de recherche internationale et l'avis sur la recherche internationale; entre également en compte la publication de la demande 18 mois après la date de priorité, etc. Il se peut qu'après l'une de ces étapes, le demandeur souhaite retirer sa demande, pour maintenir le secret.

\section{Références}

Dehon, C. et B. van Pottelsberghe (2003), «Implementing a Forecasting Methodology for PCT Applications at WIPO », Hitotsubashi University IIR, IIR Working Paper.

Dernis, H., D. Guellec et B. van Pottelsberghe (2001), " Compter les brevets pour comparer les performances technologiques entre pays ", STI Revue $n^{\circ} 27$, Direction de la science, de la technologie et de l'industrie, OCDE, Paris.

Dernis, H. (2007), " Nowcasting Patent Indicators », OECD Science, Technology and Industry Working Paper 2007/3, Direction de la science, de la technologie et de l'industrie, OCDE, Paris, www.oecd.org/sti/working-papers.

Grupp, H., G. Münt et U. Schmoch (1996), « Assessing Different Types of Patent Data for Describing High-technology Export Performance », in OCDE (éd.), Innovation, Patents and Technological Strategies, OECD, Paris, pp. 271-284. 
Hall, B.H., Z. Griliches et J.A Hausman (1986), « Patents and R\&D: Is There A Lag? ", NBER Working Papers 1454, National Bureau of Economic Research, Inc.

Hausman, J., B.H. Hall et Z. Griliches (1984), « Econometric Models for Count Data with an Application to the Patents-R\&D Relationship ", Econometrica, Econometric Society, vol. 52 (4), pp. 909-38.

Hinze, S. et U. Schmoch (2004), « Opening the Black Box. Analytical approaches and their impact on the outcome of statistical patent analyses " in W. Glänzel, H. Moed et U. Schmoch (éd.) (2004): Handbook of Quantitative Science and Technology Research: The Use of Publication and Patent Statistics in Studies on R\&D Systems, Kluwer Academic Publishers, Dordrecht/Boston/Londres, pp. 215-235.

Khan, M. et H. Dernis (2005), « Impact of Patent Co-Operation Treaty Data on EPO Patent Statistics and Improving the Timeliness of EPO Indicators ", OECD Science, Technology and Industry Working Paper 2005/2, Direction de la science, de la technologie et de l'industrie, OCDE, Paris, www.oecd.org/sti/working-papers.

OCDE (2005), « Compendium of Patent Statistics 2005 », OCDE, Paris.

OCDE (2007), « Compendium of Patent Statistics 2007 », OCDE, Paris.

Schmoch, U. (1999), "Impact of International Patent Applications on Patent Indicators », Research Evaluation, vol. 8, $n^{\circ}$ 2, pp. 119-131. 


\section{ANNEXE 4.A1}




\section{Encadré 4.A1.1. Autres définitions des familles de brevets}

Dans le domaine des brevets, une famille de brevets peut être définie de plusieurs façons pour établir une relation entre un document brevet et son ou ses document(s) prioritaire(s) comme stipulé dans la Convention de Paris. Trois définitions de familles de brevets seront décrites ci-après, basées sur l'exemple suivant :

\begin{tabular}{llll}
\hline Document D1 & Priorité P1 & & \\
Document D2 & Priorité P1 & Priorité P2 & \\
Document D3 & Priorité P1 & Priorité P2 & Priorité P3 \\
Document D4 & & Priorité P2 & Priorité P3 \\
\hline Document D5 & & & \\
\hline
\end{tabular}

Définition 1 : tous les documents qui sont directement ou indirectement liés via un document prioritaire appartiennent à la même famille de brevets. C'est la définition utilisée par l'INPADOC. Dans ce cas, les documents D1 à D5 appartiennent à la même famille de brevets P1.

\begin{tabular}{cccc}
\hline Famille P1 & & & \\
Document D1 & Priorité P1 & & \\
Document D2 & Priorité P1 & Priorité P2 & \\
Document D3 & Priorité P1 & Priorité P2 & Priorité P3 \\
Document D4 & & Priorité P2 & Priorité P3 \\
\hline Document D5 & & & \\
\hline
\end{tabular}

Définition 2 : tous les documents ayant au moins une priorité en commun appartiennent à la même famille de brevets. C'est la définition utilisée par esp@cenet pour obtenir la liste des documents de la famille en indiquant le numéro de priorité dans le champ correspondant du formulaire de recherche. On obtient ainsi l'affichage de la liste des documents de la famille (liste des réponses). Dans cette hypothèse, les documents D1, D2 et D3 appartiennent à la famille P1, les documents D2, D3 et D4 à la famille P2 et les documents D4 et D5 à la famille P3.

\begin{tabular}{llll}
\hline & Famille P1 & Famille P2 & Famille P3 \\
Document D1 & Priorité P1 & & \\
Document D2 & Priorité P1 & Priorité P2 & \\
Document D3 & Priorité P1 & Priorité P2 & Priorité P3 \\
Document D4 & & Priorité P2 & Priorité P3 \\
\hline Document D5 & & & \\
\hline
\end{tabular}




\section{Encadré 4.A1.1. Autres définitions des familles de brevets (suite)}

Définition 3 : tous les documents ayant exactement la même ou les mêmes priorités par combinaison appartiennent à la même famille de brevets. C'est la définition utilisée par esp@cenet pour sélectionner le document de référence qui s'affiche dans la « vue du document " pris dans la liste de documents de la famille donnée en résultat (liste des réponses). Dans ce cas, le document D1 n'appartient qu'à la famille P1, les documents D2 et D3 appartiennent aux familles P1-P2, le document D4 n'appartient qu'aux familles P2-P3 et le document D5 n'appartient qu'à la famille P3.

\begin{tabular}{|c|c|c|c|c|}
\hline Document D1 & Priorité P1 & & & Famille P1 \\
\hline Document D2 & Priorité P1 & Priorité P2 & & Famille P1-P2 \\
\hline Document D3 & Priorité P1 & Priorité P2 & & Famille P1-P2 \\
\hline Document D4 & & Priorité P2 & Priorité P3 & Famille P2-P3 \\
\hline Document D5 & & & Priorité P3 & Famille P3 \\
\hline
\end{tabular}

Notes : Après une recherche, tous les documents répertoriés dans la liste des réponses seront affichés individuellement pour s'assurer qu'aucune information n'est omise. Le fait de n'afficher que le premier document de la liste des réponses n'est le plus souvent pas suffisant. La définition 1 correspond aux familles Inpadoc. La définition 2 correspond aux familles esp@cenet. La définition 3 correspond aux équivalents esp@cenet.

Source : Office européen des brevets. 


\section{Tableau 4.A1.1. Écarts dans les comptages de brevets (OEB) selon la référence choisie, 2000}

\begin{tabular}{|c|c|c|c|c|c|c|c|c|}
\hline \multirow{3}{*}{ Références } & \multicolumn{4}{|c|}{ Nombre de brevets } & \multicolumn{4}{|c|}{ Parts dans les pays de l'OCDE } \\
\hline & \multicolumn{2}{|c|}{ Délivrances } & \multicolumn{2}{|c|}{ Demandes } & \multicolumn{2}{|c|}{ Délivrances } & \multicolumn{2}{|c|}{ Demandes } \\
\hline & Priorité & Délivrance & Priorité & Demandes & Priorité & Délivrance & Priorité & Demandes \\
\hline Allemagne & 9057 & 5585 & 25221 & 24409 & 29.02 & 20.58 & 17.25 & 18.16 \\
\hline Australie & 103 & 146 & 1850 & 1706 & 0.33 & 0.54 & 1.26 & 1.27 \\
\hline Autriche & 554 & 264 & 1393 & 1257 & 1.78 & 0.97 & 0.95 & 0.94 \\
\hline Belgique & 404 & 321 & 1490 & 1470 & 1.29 & 1.18 & 1.02 & 1.09 \\
\hline Canada & 394 & 308 & 2609 & 2353 & 1.26 & 1.13 & 1.78 & 1.75 \\
\hline Corée & 270 & 163 & 2620 & 1985 & 0.86 & 0.60 & 1.79 & 1.48 \\
\hline Danemark & 312 & 199 & 1196 & 1051 & 1.00 & 0.73 & 0.82 & 0.78 \\
\hline Espagne & 305 & 155 & 1058 & 963 & 0.98 & 0.57 & 0.72 & 0.72 \\
\hline États-Unis & 5718 & 7074 & 49389 & 45278 & 18.32 & 26.07 & 33.77 & 33.69 \\
\hline Finlande & 385 & 272 & 1814 & 1755 & 1.23 & 1.00 & 1.24 & 1.31 \\
\hline France & 2601 & 2170 & 8439 & 8184 & 8.33 & 8.00 & 5.77 & 6.09 \\
\hline Grèce & 10 & 8 & 74 & 62 & 0.03 & 0.03 & 0.05 & 0.05 \\
\hline Hongrie & 41 & 22 & 207 & 177 & 0.13 & 0.08 & 0.14 & 0.13 \\
\hline Irlande & 52 & 33 & 288 & 322 & 0.17 & 0.12 & 0.20 & 0.24 \\
\hline Islande & 7 & 3 & 43 & 41 & 0.02 & 0.01 & 0.03 & 0.03 \\
\hline Italie & 1559 & 1025 & 4493 & 4303 & 5.00 & 3.78 & 3.07 & 3.20 \\
\hline Japon & 4989 & 5497 & 24432 & 20909 & 15.98 & 20.26 & 16.71 & 15.56 \\
\hline Luxembourg & 39 & 17 & 102 & 84 & 0.12 & 0.06 & 0.07 & 0.06 \\
\hline Mexique & 7 & 6 & 103 & 103 & 0.02 & 0.02 & 0.07 & 0.08 \\
\hline Norvège & 139 & 101 & 640 & 565 & 0.44 & 0.37 & 0.44 & 0.42 \\
\hline Nouvelle-Zélande & 30 & 23 & 337 & 275 & 0.10 & 0.08 & 0.23 & 0.20 \\
\hline Pays-Bas & 839 & 749 & 3908 & 3474 & 2.69 & 2.76 & 2.67 & 2.58 \\
\hline Pologne & 16 & 10 & 121 & 106 & 0.05 & 0.04 & 0.08 & 0.08 \\
\hline Portugal & 14 & 5 & 59 & 38 & 0.04 & 0.02 & 0.04 & 0.03 \\
\hline République slovaque & 3 & 3 & 39 & 34 & 0.01 & 0.01 & 0.03 & 0.03 \\
\hline République tchèque & 27 & 7 & 107 & 123 & 0.09 & 0.03 & 0.07 & 0.09 \\
\hline Royaume-Uni & 1653 & 1582 & 7769 & 7320 & 5.30 & 5.83 & 5.31 & 5.45 \\
\hline Suède & 666 & 556 & 3269 & 3101 & 2.13 & 2.05 & 2.24 & 2.31 \\
\hline Suisse & 1005 & 832 & 3081 & 2887 & 3.22 & 3.07 & 2.11 & 2.15 \\
\hline Turquie & 13 & 3 & 90 & 74 & 0.04 & 0.01 & 0.06 & 0.06 \\
\hline OCDE & 31210 & 27139 & 146242 & 134410 & 100.00 & 100.00 & 100.00 & 100.00 \\
\hline
\end{tabular}

Note: Les comptages de brevets sont effectués sur la base du pays de l'inventeur et des comptages fractionnaires.

Source: OCDE, base de données sur les brevets. 
Tableau 4.A1.2. Part des pays dans les demandes OEB selon divers critères d'attribution

\begin{tabular}{|c|c|c|c|c|c|c|}
\hline & \multicolumn{2}{|c|}{ Pays de priorité } & \multicolumn{2}{|c|}{ Pays de l'inventeur } & \multicolumn{2}{|c|}{ Pays du demandeur } \\
\hline & 1990-02 & $2000-02$ & 1990-02 & $2000-02$ & 1990-02 & $2000-02$ \\
\hline Allemagne & 19.91 & 19.40 & 17.93 & 17.21 & 17.64 & 16.79 \\
\hline Australie & 0.80 & 0.63 & 1.06 & 1.27 & 0.99 & 1.16 \\
\hline Autriche & 0.73 & 0.87 & 1.02 & 0.99 & 0.95 & 0.82 \\
\hline Belgique & 0.16 & 0.38 & 0.96 & 1.00 & 0.76 & 0.82 \\
\hline Canada & 0.33 & 0.20 & 1.20 & 1.86 & 1.12 & 1.69 \\
\hline Corée & 1.59 & 0.27 & 0.29 & 2.22 & 0.30 & 2.22 \\
\hline Danemark & 0.62 & 0.52 & 0.71 & 0.82 & 0.72 & 0.78 \\
\hline Espagne & 0.52 & 0.39 & 0.50 & 0.80 & 0.45 & 0.66 \\
\hline États-Unis & 36.13 & 31.87 & 31.98 & 33.14 & 32.80 & 33.99 \\
\hline Finlande & 0.89 & 0.74 & 0.90 & 1.20 & 0.88 & 1.42 \\
\hline France & 6.18 & 8.07 & 7.70 & 5.89 & 7.49 & 5.70 \\
\hline Grèce & 0.05 & 0.03 & 0.06 & 0.06 & 0.05 & 0.05 \\
\hline Hongrie & 0.07 & 0.09 & 0.14 & 0.13 & 0.12 & 0.10 \\
\hline Irlande & 0.11 & 0.08 & 0.13 & 0.22 & 0.13 & 0.25 \\
\hline Islande & 0.02 & 0.01 & 0.01 & 0.03 & 0.01 & 0.03 \\
\hline Italie & 2.84 & 3.52 & 3.51 & 3.18 & 3.26 & 2.84 \\
\hline Japon & 19.56 & 20.37 & 18.16 & 16.69 & 18.00 & 16.63 \\
\hline Luxembourg & 0.05 & 0.05 & 0.05 & 0.06 & 0.10 & 0.12 \\
\hline Mexique & 0.01 & 0.01 & 0.02 & 0.09 & 0.02 & 0.07 \\
\hline Norvège & 0.26 & 0.22 & 0.36 & 0.43 & 0.37 & 0.39 \\
\hline Nouvelle-Zélande & 0.14 & 0.07 & 0.12 & 0.23 & 0.11 & 0.21 \\
\hline Pays-Bas & 0.80 & 1.43 & 2.32 & 2.84 & 2.93 & 3.44 \\
\hline Pologne & 0.05 & 0.02 & 0.04 & 0.10 & 0.02 & 0.08 \\
\hline Portugal & 0.02 & 0.01 & 0.02 & 0.04 & 0.01 & 0.04 \\
\hline République slovaque & 0.01 & 0.00 & 0.00 & 0.02 & 0.00 & 0.02 \\
\hline République tchèque & 0.05 & 0.04 & 0.05 & 0.08 & 0.04 & 0.06 \\
\hline Royaume-Uni & 5.72 & 6.85 & 6.32 & 5.21 & 5.71 & 4.34 \\
\hline Suède & 1.64 & 1.60 & 1.85 & 2.01 & 1.83 & 2.28 \\
\hline Suisse & 0.73 & 2.28 & 2.60 & 2.12 & 3.17 & 2.95 \\
\hline Turquie & 0.03 & 0.00 & 0.00 & 0.07 & 0.00 & 0.06 \\
\hline OCDE & 100.00 & 100.00 & 100.00 & 100.00 & 100.00 & 100.00 \\
\hline
\end{tabular}

Note : Les comptages de brevets sont effectués sur la base de la date de priorité et de comptages fractionnaires.

Source: OCDE, base de données sur les brevets. 



\section{Chapitre 5}

\section{Classification des brevets} en fonction de différents critères 


\subsection{Introduction}

Pour bon nombre d'utilisations de données sur les brevets, qu'il s'agisse de recherche ou d'analyse des politiques, il est nécessaire de rapporter ces données à une unité d'analyse parlante, ou de les classer en fonction de critères particuliers. Associer ou classifier ainsi les brevets permet de déceler des informations sur ces unités particulières ou encore sur la pertinence économique ou sociale de certaines variables. L'analyse peut nécessiter que les brevets considérés soient rapportés à l'entité qui les a déposés, à l'individu auteur de l'invention, à un domaine technologique précis, à un secteur d'activité, à une région du monde ou à un type d'institution.

Néanmoins, les données sur les brevets ne permettent pas d'exploiter immédiatement ce type d'informations. Il faut les déduire en "nettoyant» les données déjà disponibles (c'est-à-dire en rectifiant les erreurs et en uniformisant la présentation) et en recoupant ces informations avec d'autres sources de données comme les listes de sociétés, les listes de domaines technologiques ou les tables de concordance (entre les codages des technologies et ceux des activités, les noms des villes et ceux des régions, etc.). Ces sources de données permettront, à leur tour, de compléter les informations contenues dans les brevets. Il s'agira d'abord identifier puis de traiter avec soin les données fournies dans les dossiers de brevets, ce qui est généralement ardu et nécessite un traitement informatique assez lourd.

Ce chapitre récapitule les principales classifications utilisées pour les brevets - à savoir les brevets par domaine technologique, par secteur d'activité, par région et par type d'institution - et décrit brièvement les approches méthodologiques communément appliquées pour leur élaboration. Il présente également les procédures générales utilisées pour associer les données des brevets aux sociétés et pour les regrouper par inventeur. Les indications contenues dans ce chapitre pourront servir de base à des améliorations ultérieures dans ce domaine.

\subsection{Domaines technologiques}

Les brevets constituent tout naturellement une source d'informations sur l'évolution de la technologie, car ils concernent principalement des inventions techniques. Bien souvent, ils représentent d'ailleurs les seules sources fiables de données. C'est le cas notamment lorsque l'on souhaite examiner de nouveaux domaines techniques émergents, non encore stabilisés (c'est-à-dire 
pour lesquels il n'existe pas encore de définition opérationnelle), qui ne sont pas pris en compte dans les enquêtes menées auprès des entreprises, etc.

Les données sur les brevets couvrent un large éventail de domaines et s'inscrivent dans la longue durée, ce qui en fait un instrument utile pour observer les technologies à long terme, déceler les percées technologiques, les fertilisations croisées entre domaines, etc. Le graphique 5.1 présente l'exemple des brevets rattachés à la technologie des piles à combustible depuis le début des années 90. Dans l'analyse du développement technologique, les données sur les brevets sont utilisées pour observer des aspects tels que :

- Les nouveaux domaines technologiques (émergence et évolution) : par exemple les polymères semi-conducteurs, les technologies d'énergie éolienne.

- Le cycle de vie des technologies (maturité des technologies) : par exemple en observant les taux de croissance annuels des dépôts de brevets sur de longues périodes afin de déceler un éventuel ralentissement des nouvelles avancées technologiques majeures (technologies matures: agriculture, véhicules à moteur, etc.).

- La fertilisation croisée (comment une technologie influence d'autres) : par exemple l'influence des technologies plasma sur l'électronique (puces de nouvelle génération), sur les technologies environnementales (lampes à plasma).

\section{Graphique 5.1. Évolution des dépôts de brevets sur les piles à combustible ${ }^{1}$, part des brevets déposés selon le PCT $^{2}, 1987-2004$}

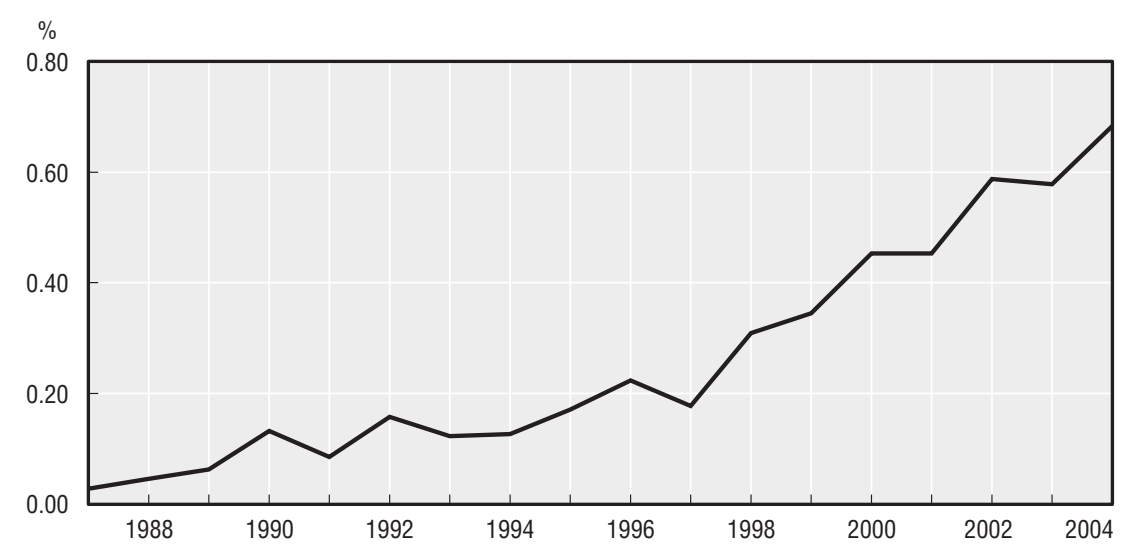

Note : Comptage des brevets par date de priorité et résidence de l'inventeur, avec comptage fractionnaire.

1. Les brevets portant sur les piles à combustible sont identifiés en utilisant les classes CIB H01M8/00-8/24, et renvoient aux demandes de brevet selon le PCT dans la phase internationale, qui désigne l'OEB.

Source : OCDE, base de données sur les brevets. 
Le document brevet renferme différents types d'informations pouvant être utilisées pour classer les brevets dans des domaines particuliers : un codage des classes techniques et différentes informations textuelles (titre, abrégé, revendications et description). D'autres informations peuvent aussi être présentes dans certains cas, par exemple le nom du déposant ou des références.

\subsubsection{Le système de classification internationale des brevets}

Pour faciliter la recherche sur l'état antérieur de la technique, les bureaux des brevets classifient les brevets en fonction de leur domaine. Ces codes figurent en première page du document brevet. Ces classifications ont été construites selon un point de vue technique afin que l'on puisse retrouver les documents qui reflètent l'état de la technique dans un domaine particulier.

Pour diffuser des informations sur les brevets dans le monde entier, on utilise un système international commun. La Classification Internationale des Brevets (CIB), né de l'Accord de Strasbourg de 1971, est une méthode reconnue au niveau international pour classer les brevets d'invention, y compris les demandes (ou dépôts) de brevet publiées, les modèles ou certificats d'utilité. Aujourd'hui, plus de 100 pays utilisent la CIB, laquelle constitue la principale, et parfois la seule, forme de classification de ces documents. Le but du système CIB est de regrouper les documents de brevets en fonction du domaine technique auquel ils se rapportent, indépendamment de la langue et de la terminologie employée.

D'après le guide CIB ( 8 édition, 2006 - CIB8), une invention est affectée à une classe CIB de par sa fonction ou nature intrinsèque, ou de par son domaine d'application. La CIB est donc un système de classification qui combine fonction et application, dans lequel l'application prime. Un brevet peut avoir plusieurs objets techniques, auquel cas il doit être affecté à plusieurs classes CIB. Les codes CIB sont publiés sur les documents de brevets ${ }^{1}$. Le système CIB est révisé périodiquement pour être amélioré et prendre en compte les évolutions techniques et électroniques. Si nécessaire, il est modifié. Avant 2006, les modifications apportées n'avaient pas d'effet rétroactif, ce qui peut poser problème en cas d'utilisation de séries antérieures. Depuis avril 2007, plus de 140 millions de classifications CIB8 ont été appliquées, dont environ $92 \%$ l'ont été de manière rétroactive sur des documents publiés avant l'entrée en vigueur de la CIB8. Les sous-groupes sont hiérarchiques. Le niveau de sous-groupe est indiqué par le nombre de points qui figurent avant le titre. Dans la $8^{\mathrm{e}}$ édition du CIB apparaissent les niveaux de base et les niveaux avancés (voir l'exemple dans le tableau 5.1).

L'OEB travaille avec l'ECLA (le système de classification européen), une version affinée de la CIB (140 000 catégories au lieu de 70000 pour la CIB). L'USPTO utilise la classification américaine des brevets, l'USPC, laquelle compte plus de 160000 subdivisions. L'un des principes de base du système USPC est que chaque classe est créée en analysant en premier lieu les revendications divulguées des brevets américains, puis, à partir de cette analyse, sont créées les différentes 


\section{Tableau 5.1. Principales caractéristiques des codes CIB (exemple)}

\begin{tabular}{llll}
\hline Nom de la division & Nombre de rubriques & Exemple de code & Intitulé de la rubrique \\
\hline Section & 8 & G & Physique \\
Sous-section & 20 & G0 & Instruments \\
Classe & 118 & G06 & Calcul, comptage \\
Sous-classe & 616 & G06F & Calculateurs numériques \\
Groupe principal & 6871 & G06F-9/000 & $\begin{array}{l}\text { Dispositifs pour la commande } \\
\text { par programmation }\end{array}$ \\
Sous-groupe & 57324 & G06F-9/06 & *Utilisant un programme emmagasiné \\
& & G06F-9/46 & **Dispositifs \\
pour la multiprogrammation
\end{tabular}

Source: Organisation Mondiale de la Propriété Intellectuelle (2006), Guide CIB, $8^{\mathrm{e}}$ édition.

divisions et subdivisions. Tous les brevets appartenant à un domaine similaire sont réunis dans de grands groupes pour former des classes. Ces classes sont alors subdivisées en unités plus petites, les sous-classes, qui se prêtent mieux aux recherches. En termes de profondeur de la classification, l'USPC donne généralement davantage d'informations sur l'invention que la CIB. La première classe USPC figurant dans un brevet est hiérarchique et constitue sa classification primaire; elle est affectée selon un ensemble bien défini de règles de classification.

Outre la CIB, le Japan Patent Office (JPO) utilise un système de classification supplémentaire, la classification FI (pour file index) avec le système F-term (fileforming term). La classification FI est une extension de la CIB et se rapproche de l'ECLA. Elle se compose d'un sous-groupe CIB suivi d'un nombre à trois chiffres appelé "symbole de subdivision CIB ". Les symboles de subdivision CIB et les symboles de discrimination de fichiers sont propres aux classes FI et sont structurés de manière hiérarchique. Le système F-term est compatible avec plusieurs perspectives différentes, à la différence de la CIB qui elle, classifie les documents selon une seule dimension technique. Chaque champ technique est déterminé par la gamme de FI, appelée " thème ${ }^{2}$, et possède une structure unique de liste F-term, laquelle contient plusieurs points de vue différents, subdivisés en plusieurs «F-terms ». Généralement, chaque document brevet contient plusieurs Fterms. Ces deux indices sont affectés par les examinateurs de brevets du JPO.

Un document brevet peut avoir un ou plusieurs codes CIB. À l'OEB, les codes CIB ne sont pas hiérarchisés, c'est-à-dire que le premier n'est pas plus important ni plus pertinent que les autres. En revanche, dans le système JPO, le premier code CIB est le code principal (il indique la classe de la technologie), ou bien il est identifié par le chiffre un (1). Les classes de brevets sont attribuées par les examinateurs; lorsqu'une procédure de dépôt est engagée, elle fait généralement l'objet d'un premier tri (par une analyse manuelle complétée par un logiciel spécialisé), afin d'être aiguillée vers l'unité d'examen appropriée. Elle est ensuite attribuée à un examinateur qui va éventuellement affiner, modifier ou 
compléter la liste de codes de l'application. On peut recourir au comptage fractionnaire pour dénombrer les brevets selon leurs classes CIB (ou domaines technologiques : groupes de classes CIB).

\subsubsection{Identification des domaines technologiques}

L'information fournie par la CIB constitue une première référence pour identifier les brevets à un domaine technologique spécifique. Elle n'est toutefois pas suffisante pour toutes les utilisations des données, dans la mesure où l'intérêt analytique ou politique n'est pas facilement attribué ou pris en compte dans la classification de brevets. C'est le cas, par exemple, des TIC (technologies de l'information et de la communication), des biotechnologies ou des nanotechnologies. Les agrégats de ce type doivent être reconstitués, sur la base des informations disponibles, à savoir le code CIB ou les données textuelles présentes.

La première étape est d'avoir une définition claire et opérationnelle du domaine technologique. Celle-ci sera complétée par des mots clés reflétant le contenu du domaine et utilisés par les ingénieurs travaillant dans le domaine concerné. La définition et les mots clés peuvent évoluer à mesure que la technologie change. On peut alors :

- Chercher les mots clés contenus dans les définitions des codes de la CIB (ou d'autres classifications techniques) et considérer comme brevets appartenant à ce domaine tous les documents qui appartiennent à un ou plusieurs des codes sélectionnés.

- Chercher les mots clés directement dans le texte des brevets (ou le titre, l'abrégé, etc.).

- Adopter une solution mixte, en recherchant les mots clés dans les codes de la CIB, en vérifiant manuellement la pertinence des résultats, etc.

Un analyste doit confirmer que l'ensemble des documents obtenus par ces méthodes correspondent bien aux critères souhaités de l'échantillon de brevets recherché.

Par exemple, à l'OEB, l'identification des brevets sur les nanotechnologies a nécessité plusieurs étapes. Un groupe de travail sur les nanotechnologies, le NTWG, a été créé en 2003. Pour commencer, ce groupe a travaillé à partir de la définition des nanotechnologies afin d'observer les tendances des brevets sur les nanotechnologies. Le NTWG a ensuite identifié les brevets concernant les nanotechnologies à l'aide de mots clés, en consultation avec les experts des nanotechnologies travaillant au sein de l'OEB, et par le biais d'examens de pairs conduits par des experts extérieurs. Les demandes de brevets de 15 pays ou organisations ont été analysées et étiquetées comme appartenant à la classe Y01N ${ }^{3}$.

L'OCDE a établi des définitions pour différents domaines techniques : les TIC, les biotechnologies, les technologies spatiales, les technologies environnementales, etc. Ces définitions comprennent : i) une définition textuelle du domaine technique, et ii) une liste de classes CIB associées. La réduction d'un 
domaine technique à une liste de classes CIB offre l'avantage d'une utilisation simple (il suffit d'identifier le code CIB d'un brevet pour l'attribuer au domaine approprié). En revanche, cela ne permet pas d'établir de distinctions à l'intérieur des codes CIB, ce qui augmente le risque de mal utiliser les documents pertinents ou d'inclure des éléments hors de propos. Le code Y01N est une réponse à ce problème, il se rapporte aux nanotechnologies et est affecté en partie par les examinateurs au cas par cas, mais étant donné son coût, il ne peut être utilisé que dans peu de domaines. Le graphique 5.1 illustre les tendances en matière de brevets concernant les piles à combustible, tandis que le graphique 5.2 montre la part des différents pays dans ce domaine technologique. Comme indiqué précédemment, les brevets fournissent des informations permettant d'observer des domaines technologiques très spécifiques et à un niveau de détail très poussé. Le graphique 5.3 présente la part des techniques liées (identifiées par le code CIB principal) aux brevets sur les piles à combustible.

Graphique 5.2. Part des pays dans les brevets sur les piles à combustible ${ }^{2}, 2000-04$

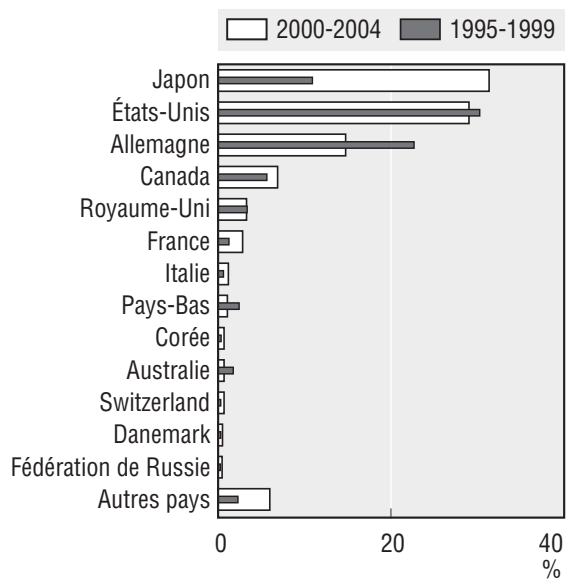

\section{Graphique 5.3. Parts des techniques apparentées ${ }^{1}$ dans les brevets sur les piles à combustible ${ }^{2}$, 2000-04}

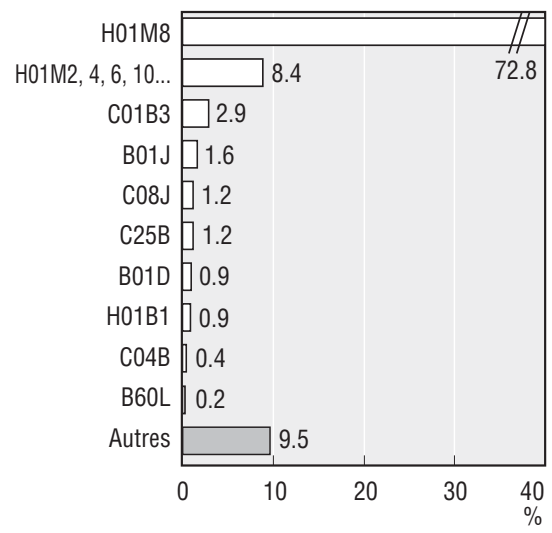

Note: Les brevets sont comptabilisés par date de priorité et résidence de l'inventeur, avec comptage fractionnaire des brevets.

1. Les brevets portant sur les piles à combustible sont identifiés aux classes CIB H01M8/00-8/24, et renvoient aux demandes de brevet selon le PCT, phase internationale, qui désigne l'OEB.

2. Différentes techniques sont comptabilisées dans le code principal CIB des brevets sur les piles à combustible : Séparation (B01D); Procédés chimiques et physiques (B01J); Équipement électrique ou propulsion des véhicules à traction électrique (B60L); Hydrogène (C01B3); Chaux; magnésie; scories; ciments (C04B); Procédés généraux pour former des mélanges (C08J); Procédés électrolytiques ou électrophorétiques (C25B); Câbles, conducteurs, isolants (H01B1); Batteries piles à combustible non classifiées (H01M2,4,6,10,12); Piles à combustible (H01M8).

Source : OCDE, base de données sur les brevets. 
L'OST-INPI/FhG-ISI (Observatoire des sciences et technologies, Institut national de la propriété intellectuelle) et l'Institut Fraunhofer-ISI (Fraunhofer Institute System und Innovationsforschung) ont proposé une ventilation des domaines techniques qui se compose d'une liste de 30 catégories techniques, correspondant à des regroupements de sous-classes CIB et couvrant l'ensemble de la classification CIB. Par rapport à la CIB elle-même, cette ventilation correspond mieux aux besoins de l'analyse à des fins politiques.

\subsubsection{La spécialisation sectorielle des pays}

L'identification des domaines technologiques et des activités à partir des brevets permet d'analyser le positionnement d'un pays en matière de technologie, par rapport à d'autres pays ou encore par rapport à la moyenne mondiale. Plus précisément, la nature sectorielle des activités de brevetage des pays s'étudie grâce aux indicateurs de spécialisation des brevets (Soete et Wyatt, 1983). L'indicateur le plus fréquemment utilisé est appelé " indice de spécialisation » ou " avantage technologique révélé » (ATR), il est défini comme la part d'un pays (i) dans les brevets se rapportant à un domaine ou une technologie particulière (d) divisée par la part de ce pays dans l'ensemble des brevets ${ }^{4}$ :

$$
\text { ATR }=\frac{\left(P_{d, i} l \sum_{d} P_{d, i}\right)}{\left(\sum_{d i} P_{d, i} l \sum_{d, i} P_{d, i}\right)}
$$

Pour un secteur donné, cet indice est égal à zéro lorsque le pays ne détient aucun brevet dans le secteur; il est égal à 1 lorsque la part du pays dans le secteur considéré est égale à sa part dans tous les domaines (auquel cas il n'existe pas de spécialisation) et progresse rapidement lorsqu'une spécialisation positive est trouvée (la limite supérieure dépend de la distribution mondiale utilisée). On utilise le logarithme de l'indice pour obtenir un nouvel indicateur avec une distribution allant de -1 à +1 . Les chiffres fondés sur les indices ATR doivent toutefois être interprétés avec prudence, surtout pour les comparaisons internationales. Un pays dont la production de brevets est très importante tendra à avoir tous ses indices ATR aux environs de 1, tandis qu'un pays qui produit peu de brevets aura une valeur très élevée dans les domaines où sa production est légèrement plus élevée que la moyenne de ce pays.

Lorsqu'ils sont calculés pour différentes périodes, les indicateurs de spécialisation montrent l'évolution des profils de spécialisation des pays dans le temps. Il faut néanmoins garder à l'esprit que les indicateurs sont relatifs à la répartition sectorielle mondiale des brevets; si un pays maintient une répartition constante de ses brevets, tandis que d'autres pays accroissent leur 
activité dans un domaine émergent, son indice de spécialisation dans ce domaine va diminuer. Le graphique 5.4 montre l'indice de spécialisation des brevets en biotechnologie et concerne les pays ayant déposé plus de 150 demandes auprès de l'OEB au cours des années 1995 à 2002.

\section{Graphique 5.4. Indice de spécialisation des brevets} dans les biotechnologies déposés à l'OEB ${ }^{1}, 2000-02$

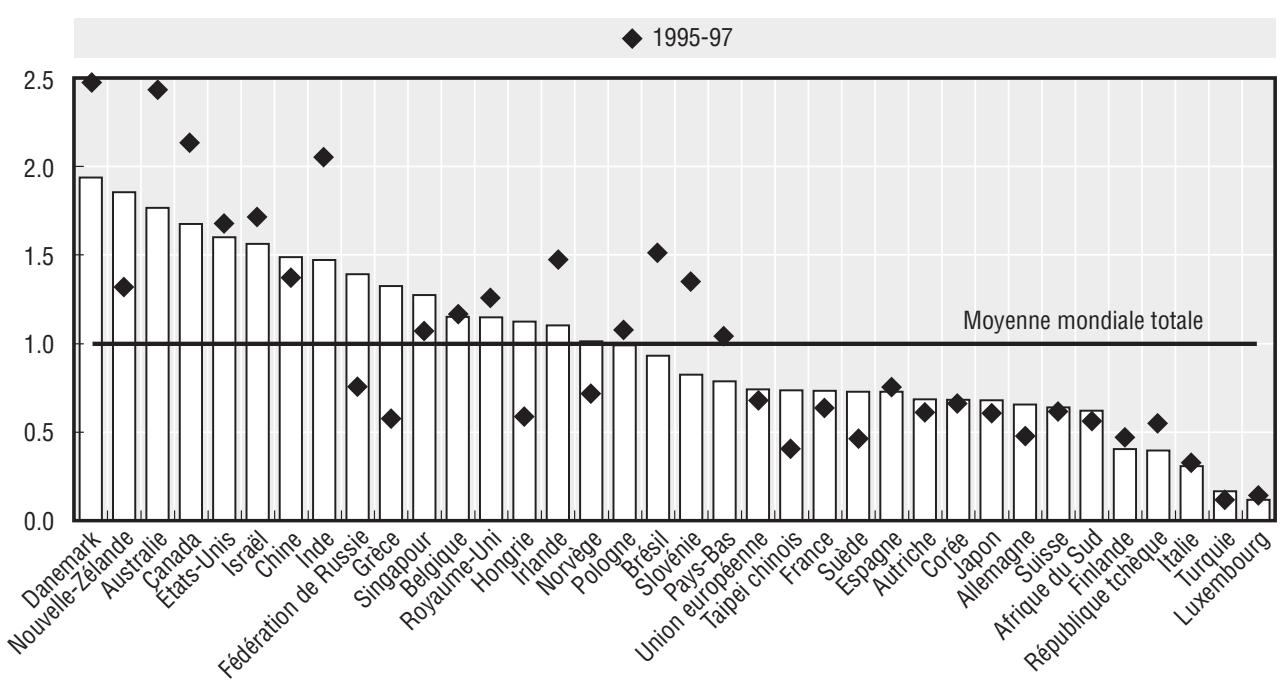

Note: Les brevets sont comptabilisés par pays de résidence de l'inventeur et date de priorité, avec comptage fractionnaire des brevets.

1. Ce graphique ne représente que les pays ou les économies comptant plus de 150 demandes l'OEB pour la période 2000-02.

Source: OCDE, base de données sur les brevets.

\subsection{Classification des secteurs d'activité}

Les brevets sont utilisés comme indicateurs de la production de la R-D ou comme intrants de l'innovation au niveau sectoriel. Il est cependant difficile de les affecter à des secteurs d'activité particuliers, car les documents sur les brevets ne permettent pas d'identifier explicitement le secteur économique auquel se rattache la technologie inhérente aux brevets. L'association des brevets aux secteurs d'activité permet de les lier à d'autres ensembles de données sectorielles, comme la base STAN de l'OCDE, et d'analyser d'importants sujets d'ordre stratégique, comme :

- La mesure du degré d'inventivité d'un secteur : en estimant les fonctions de production de savoir au niveau sectoriel, avec les intrants (notamment la R-D) à droite, les productions (indicateurs fondés sur les brevets) à gauche (par exemple, Pavitt, 1984; Ulku, 2007). 
- La spécialisation sectorielle des pays, en rapport avec la spécialisation des échanges et de la production (par exemple, Dosi et al., 1990; Malerba et Montobio, 2003).

- Les transferts de technologie intersectoriels (en utilisant par exemple les citations de brevets associées aux secteurs d'activité pourvoyeur et utilisateur de la technologie).

L'attribution de brevets aux branches d'activité peut se faire comme suit :

- Attribution directe, par examen au cas par cas du brevet.

- Attribution au brevet du code d'industrie de l'entité déposante (entreprise).

- Établissement, avec l'aide d'experts, d'une correspondance ex ante entre les classes CIB et les branches, puis intégration dans un tableau de concordance.

Dans certains cas, les différentes méthodes sont combinées, pour maximiser la quantité d'informations intégrée dans le processus.

Depuis une vingtaine d'années, plusieurs méthodes de comptage des brevets par branche ont été élaborées. Comme l'expliquent Schmoch et al. (2003), une concordance fiable doit satisfaire les conditions suivantes : i) une comparabilité internationale - adaptabilité aux autres classifications par branche; ii) un niveau de ventilation adéquat - permettant une décomposition à rebours des branches selon les domaines technologiques; iii) une base empirique solide - cohérence avec les tendances de l'activité technologique et la production des pays; et iv) elle doit être facilement applicable à des problèmes spécifiques.

On utilise deux critères différents pour attribuer les brevets aux branches d'activité : i) les brevets peuvent être rattachés au secteur industriel d'origine (secteur de l'activité principale de l'entreprise à l'origine de l'invention ou de la demande); ou ii) au secteur d'utilisation (branche principale à laquelle appartient le produit incorporant l'invention).

La plupart des tableaux de concordance existant appliquent la première méthode. Cela étant, ces classifications pose de nombreuses difficultés, car toutes les inventions ne sont pas nécessairement attribuables à un secteur d'activité particulier; le plus souvent, elles peuvent concerner plusieurs branches d'activité. Les grandes entreprises, en particulier, brevètent dans de nombreux domaines ne correspondant pas nécessairement à leur domaine d'activité économique principal. Quant aux petites entreprises, plus spécialisées, leur domaine d'activité peut ne figurer dans aucune base de données. Les tableaux de concordance doivent être réactualisés périodiquement car la classification des brevets et des industries évolue avec le temps.

L'une des premières tentatives de construction d'un tableau de concordance par secteurs d'activité pour les brevets est la « Concordance de Yale », construite par Evenson, Putnam et Kortum (1991) sur la base de la classification sectorielle établie 
par l'OPIC (Office de la propriété intellectuelle du Canada). Entre 1972 et 1995, l'OPIC a attribué simultanément un code CIB, un code par industrie de fabrication (IOM) et par secteur d'utilisation (SOU) à plus de 300000 brevets délivrés.

Autre tentative, la "Concordance de l'OTAF », réalisée par l'USPTO, entre le système américain de classification des brevets (USPC) et la SIC (Standard Industrial Classification), créée en 1974. Elle s'appuie sur un examen manuel et une cartographie des catégories USPC, associées à un ensemble limité de domaines de produits axés sur les secteurs d'activité, selon la SIC 1972. Il s'agit des subdivisions SIC de niveau élevé, généralement à deux ou trois chiffres (41 secteurs d'activité). Basée sur la nomenclature des industries de fabrication, elle est actualisée régulièrement, en général chaque année, pour suivre les changements et les révisions annuelles de l'USPC. Des travaux sont entrepris pour adapter cette concordance au Système de classification des industries de l'Amérique du Nord (NAICS). On mentionnera en outre, dans ce domaine, la concordance proposée par Johnson (2002) basée sur les données de l'Office de la propriété intellectuelle du Canada. Il s'agit notamment de liens avec les technologies, en fonction des correspondances possibles, vers environ 115 secteurs de fabrication et d'utilisation.

Une table de concordances plus récente est celle conçue par Schmoch et al. (2003) de l'Institut Fraunhofer de recherche sur les systèmes et l'innovation, l'Observatoire des sciences et des techniques (OST) et l'Unité de recherche sur les sciences et les politiques de l'Université du Sussex (SPRU). Le secteur d'activité économique des sociétés est utilisé pour classifier les technologies dans les secteurs d'activité ${ }^{5}$. La méthode s'est élaborée en quatre étapes. Premièrement, un ensemble de secteurs industriels, défini par les codes NACE et CITI (à 2 chiffres) a été choisi comme point de départ. Deuxièmement, des experts techniques ont associé 625 sous-classes de la CIB à des catégories technologiques (44 domaines) et à des catégories industrielles en fonction des caractéristiques de fabrication des produits. Troisièmement, les rattachements techniques et industriels ont été comparés en analysant les activités de brevetage par domaine technologique de 3400 grandes entreprises productrices de brevets, classées par branche industrielle ( 44 branches). Cette évaluation a conduit à l'élaboration d'une matrice de transfert ou de concordance entre classifications par technologie et par branche. Quatrièmement, la validité et l'utilité pratique de la concordance ont été vérifiées en comparant les structures par pays ainsi obtenues (par exemple les similitudes dans la distribution d'une technologie donnée entre branches et à l'intérieur des branches, par pays et dans le temps). Le projet était parrainé par Eurostat. Il est repris par l'OCDE pour la base de données ANPAT, qui est la partie de la base STAN consacrée aux brevets (STAN comprend également des données sectorielles sur la valeur ajoutée, l'emploi, la R-D, etc., pour 20 industries et à partir de l'année 1971). 
Le graphique 5.5 présente la relation, établie à partir de la table de concordances, entre l'activité de brevetage et les dépenses de R-D (moyennes OCDE) pour les industries manufacturières. Les branches à forte intensité de R-D comme les industries pharmaceutique et médicale, les instruments de précision et d'optique, sont celles qui suscitent le plus de brevets. À l'inverse, on observe une activité technologique plus faible, tant en termes de R-D que de brevetage, dans les industries liées au textile, au cuir, au bois et au papier.

\section{Graphique 5.5. Brevets par branche et R-D des entreprises ${ }^{1,2}$, dépôts selon le PCT, 2002-04}

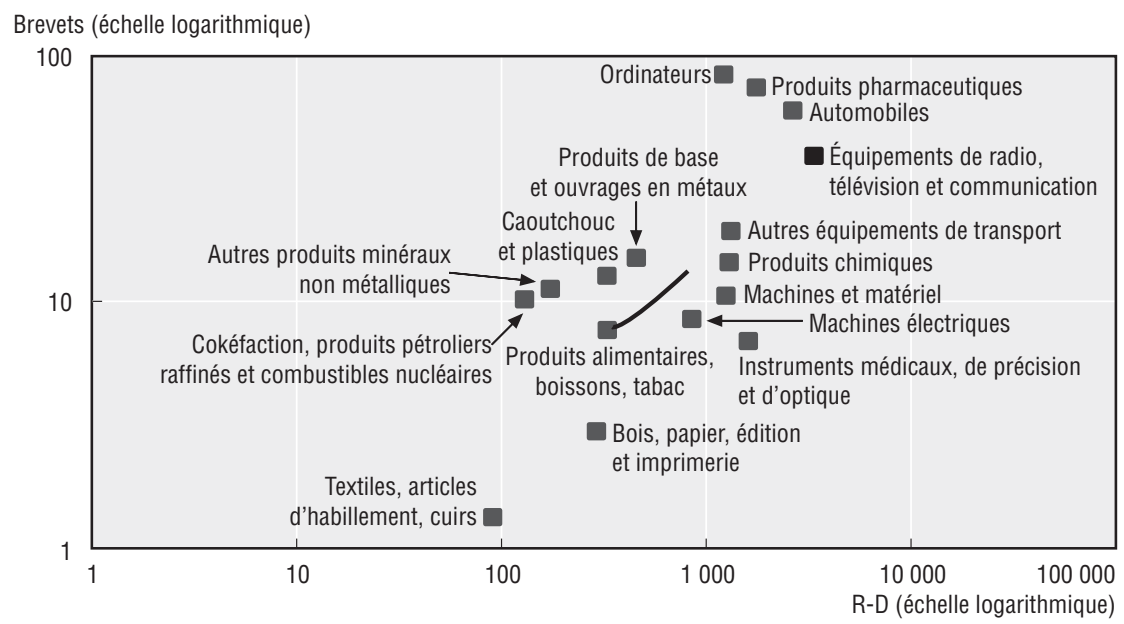

Note: Le comptage des brevets tient compte de la date de priorité, avec comptage fractionnaire.

1. Les demandes de brevets selon le Traité de coopération en matière de brevets (PCT) au niveau international, désignant l'Office européen des brevets (OEB).

2. Dépenses moyennes de R-D des entreprises en 1999-2000, en millions USD (2000) en parités de pouvoir d'achat et brevets par activité en 2002-04 dans les pays de l'OCDE.

Source: OCDE, base de données sur les brevets.

\subsection{Classification régionale}

Pour répondre aux besoins des décideurs politiques régionaux et nationaux, il est important de décrire et de comprendre les profils régionaux de l'innovation; sur le plan régional, l'intérêt est d'établir des références et des comparaisons, tandis que sur le plan national, il est de saisir l'importance des politiques nationales d'innovation. La répartition des brevets par région permet de répondre à des questions concernant l'action publique, telles que :

- La comparaison des performances et des profils technologiques des régions.

- L'importance de la proximité géographique dans l'innovation (Jaffe et al., 1993; Audretsch et Feldman, 1996). 
- L'analyse de la répartition spatiale (ou de la concentration) de l'activité d'innovation et de production entre les régions (par exemple, Paci et Usai, 2000).

- Les interactions et la coopération technologiques au sein des régions et entre régions (par exemple, Breschi et Lissoni, 2001).

La première page d'un brevet fournit l'adresse des inventeurs et des déposants. Ces informations comprennent la ville, la région et le code postal et permettent de rattacher les brevets à une région particulière (celle de l'inventeur ou de celui qui dépose le brevet en utilisant des tables de référence (codes postaux, noms de ville, etc.). La régionalisation des données sur les brevets dépend des détails et de la qualité de l'information contenus dans l'adresse; pour certains pays, ces informations ne sont pas très détaillées et pas toujours harmonisées d'un bureau des brevets à l'autre. Pour palier ce problème d'information souvent parcellaire et parfois inexistante, il faut appliquer des algorithmes complexes qui identifient les données pertinentes et les associent aux informations disponibles dans des bases de données régionales spécialisées. Par exemple, les brevets de l’USPTO ne comprennent généralement pas le code postal (ZIP) de l'inventeur, mais seulement le nom de la ville et parfois le code de l'état ${ }^{6}$. Pour affecter ces brevets à une région, il faut utiliser le nom de la ville, sachant qu'il faut s'attendre à des problèmes, par exemple le fait que plusieurs villes peuvent porter le même nom.

Les régions sont définies de manière standardisée. L'OCDE utilise la classification TL (niveaux territoriaux), laquelle comporte différents niveaux d'agrégation (le niveau 2 comprend environ 300 macro-régions; le niveau 3 comprend 2300 régions, à savoir les zones économiques du Bureau of Economic Analysis (BEA) aux États-Unis, les préfectures au Japon, les départements en France). Dans les pays de l'UE, les régions sont définies par la NUTS (Nomenclature des unités territoriales statiques), une classification officielle de la Commission européenne. L'OCDE a compilé des bases de données sur les brevets (TCB, OEB) au niveau TL3 (voir Maraut et al., 2008) ${ }^{7}$. Le graphique 5.6 présente les régions qui brevètent le plus dans le domaine des TIC.

Dès lors qu'on utilise les données géographiques sur les brevets, il faut garder à l'esprit deux aspects particuliers. D'abord, s'agissant des inventeurs, il faut prendre garde de ne pas considérer un niveau trop détaillé pour certains grands centres urbains. La résidence de l'inventeur peut avoir un code postal différent du laboratoire ou il/elle travaille (c'est-à-dire, dans une zone voisine). Les différents co-inventeurs d'une même invention peuvent résider dans différentes zones de la même (grande) ville, tout en travaillant au même endroit. Par conséquent, pour les grands centres urbains qui se composent de sous-zones détaillées, il peut être préférable de travailler à un niveau plus agrégé (par exemple au niveau 2 au lieu du niveau 3). En Europe, c'est le cas de 
la région parisienne et du grand Londres. Ensuite, s'agissant des déposants, une demande de brevet peut être déposée par une société affiliée à l'entreprise, ou co-déposée par l'entreprise et une de ses filiales. Dans ce cas, l'adresse de la société affiliée apparaîtra et ne correspondra pas à la localisation de l'entité qui a le contrôle effectif du brevet. Pour résoudre ce problème, il faut consolider le contrôle des sociétés au niveau du groupe.

\section{Graphique 5.6. Brevets ICT par région en Europe, aux États-Unis et au Japon ${ }^{1,2,3}$}

(a) Le nombre de demandes de brevets selon le PCT et (b) les demandes d'applications selon le PCT au regard de la population active en 2004
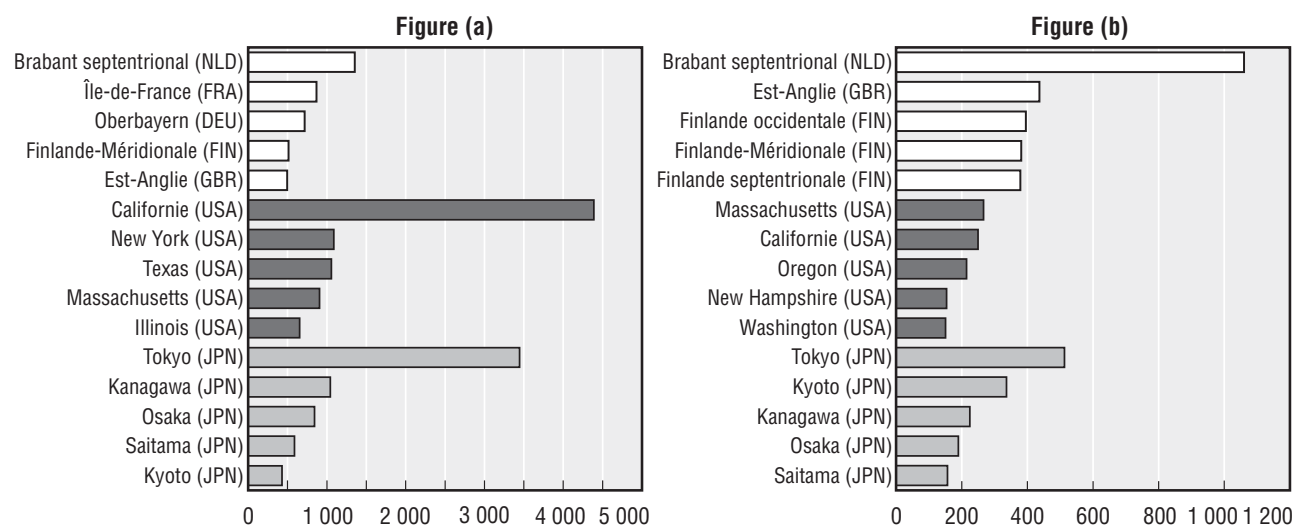

Note : Le comptage des brevets tient compte de la date de priorité, de la région de résidence de l'inventeur, avec comptage fractionnaire.

1. Seuls les pays comptant plus de 100 demandes de brevet selon le PCT en 2004 sont représentés.

2. Les pays dans lesquels $60 \%$ des adresses des inventeurs sont affectées à des régions sont inclus.

3. Seules les régions comptant plus de 100 demandes de brevets selon le PCT en 2004 sont représentées. Les brevets TIC sont identifiés par la Classification internationale des brevets (CIB).

Source: OCDE, base de données sur les brevets.

\subsection{Type d'institution}

Le type d'institution d'un titulaire de brevet est défini par son statut juridique : il peut s'agir d'un individu, d'une société (secteur privé), d'une instance gouvernementale, d'une université ou d'un hôpital. Identifier l'activité de brevetage des universités et des établissements public permet d'observer des aspects tels que :

- L'impact de certaines politiques sur le dépôt de brevets par les universités (par exemple la loi Bayh-Dole aux États-Unis, ou des politiques de ce type dans d'autres pays; voir Mowery et al., 2001).

- Les profils de coopération dans la recherche entre universités, centres de recherche publics et entreprises publiques (voir par exemple, Cassiman et Veugelers, 2005). 
Les données sur les brevets peuvent être associées à d'autres données comme celles sur la R-D, à condition que la liste des secteurs institutionnels des deux sources d'informations soit compatible.

Les méthodes appliquées pour affecter des catégories institutionnelles aux brevets utilisent des algorithmes conçus pour identifier l'information pertinente à partir du champ " nom » des brevets, lequel peut donner des indices sur le type d'institution (voir tableau 5.1). Ces indices peuvent se rapporter à une partie du nom, ou à certains mots particuliers (par exemple " national ") et/ou à des termes propres à des formes juridiques spécifiques (par exemple "S.A."). Si ces indices sont identifiables de manière systématique, ils sont alors intégrés à un script qui, à lui seul, permettra une affectation automatisée des codes sectoriels.

Van Looy et al. (2006) ont récemment élaboré pour Eurostat une méthode reposant sur cette approche (tableau 5.2). Comme le Manuel de Frascati de l'OCDE $^{8}$, cet algorithme permet l'affectation de brevets : i) à un individu; ii) à une société commerciale; iii) au secteur public; iv) à l'enseignement supérieur; v) à un hôpital; vi) à une organisation à but non lucratif ${ }^{9}$. La procédure analytique utilise à la fois une logique fondée sur des principes et une logique de base empirique. La première repose sur l'hypothèse que les informations correspondant au nom du titulaire du brevet peuvent contenir des mots clés sur le type d'institution à laquelle il appartient, ce qui peut déboucher sur un ensemble de règles pour l'affectation des codes de secteur (ou de type d'institution). Toutefois, dans la pratique, comme l'ont constaté les auteurs, cette approche algorithmique peut s'avérer incomplète et peu précise. On se heurte souvent à l'absence d'indices ou à la coexistence de plusieurs indices suggérant des secteurs différents. Pour

\section{Tableau 5.2. Exemples de mots clés ou d'indices utilisés} pour identifier le secteur de brevets

\begin{tabular}{|c|c|}
\hline Type d'institution & Mots clés \\
\hline (1) Individu & 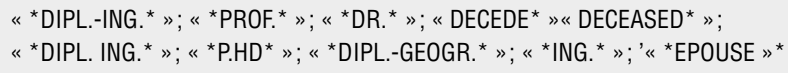 \\
\hline (2) Entreprise privée & 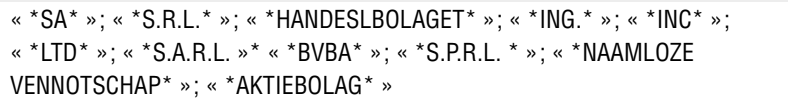 \\
\hline (3) Enseignement supérieur & 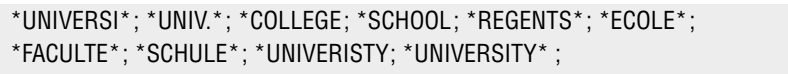 \\
\hline (4) Hôpital & 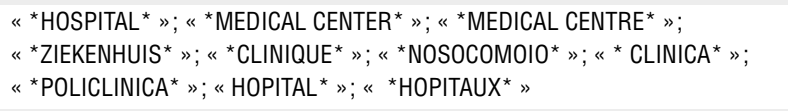 \\
\hline $\begin{array}{l}\text { (5) Organisations publiques } \\
\text { et privées à but non lucratif }\end{array}$ & 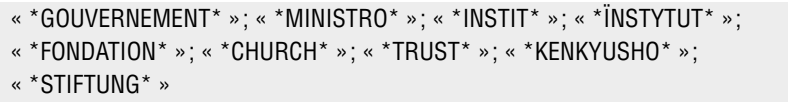 \\
\hline
\end{tabular}

Source : Van Looy et al. (2006). 
remédier à cette situation, on introduit une deuxième couche qui a, quant à elle, un fondement empirique. La conditionnalité permet de diminuer le nombre d'affectations de plusieurs types d'institutions.

Il n'est pas toujours évident de faire correspondre les caractéristiques du nom aux différentes catégories dès lors qu'il s'agit de certains types d'organisations. Par exemple, un hôpital peut être classé comme entreprise commerciale ou comme organisation privée à but non lucratif ou comme établissement d'enseignement supérieur, selon le mode de gouvernance qui le caractérise. Le secteur auquel devrait être rattachée une organisation donnée n'est pas toujours évident si l'on se fonde uniquement sur les informations contenues dans le champ nom du système de brevets. Pour y remédier, les auteurs ont introduit différents types de règles; outre les règles génériques qui associent plusieurs déposants de brevets à un secteur, d'autres règles ont été ajoutées pour cibler précisément certaines organisations. C'est l'approche suivie par Eurostat et par l'OCDE.

Il faut noter que l'utilisation des universités comme déposant des brevets issus de travaux universitaires donne un comptage incomplet. Les inventions faites par des chercheurs universitaires ne sont pas obligatoirement brevetées par l'université elle-même. Elles peuvent l'être par le chercheur ou par une société qui l'a financé. Pour repérer ce type d'inventions, il faut identifier les inventeurs universitaires (leurs noms et adresses). En mettant en correspondance les noms des inventeurs et ceux des auteurs (à partir des listes de chercheurs) il apparaît que, dans de nombreux pays, on ne peut pas identifier la moitié au moins des chercheurs à l'origine de brevets issus d'universités (Noyens et al., 2003). Autre stratégie possible : identifier l'université ou l'établissement de rattachement par l'adresse de l'inventeur; pour certains pays, cette stratégie accroît d'environ 10 \% le nombre de brevets provenant de l'enseignement supérieur.

\subsection{Brevets provenant d'entreprises commerciales}

L'attribution d'un brevet à l'entité qui le possède est une étape clé dans un grand nombre de travaux statistiques et analytiques portant sur les brevets. Cela permet de reconstituer le portefeuille de brevets des sociétés, ce qui sert notamment :

- À compiler des classifications de brevets par branche, par domaine technologique, par région, par type d'institution, etc.

- À analyser la stratégie des entreprises en matière de brevets (moment et orientation choisis pour déposer les brevets, par rapport à leurs concurrents).

En associant les informations sur les brevets à d'autres données concernant l'entreprise, comme la R-D, l'innovation, la valeur boursière, etc., on peut rattacher la stratégie de l'entreprise en matière de technologies et de brevets à 
d'autres caractéristiques : quel est l'impact des brevets sur la valeur boursière? Quelle est l'efficacité de la R-D (en termes de nombre de brevets)?

Les nom et adresse du titulaire du brevet sont publiés dans le document brevet; toutefois, l'attribution d'un brevet à une entité donnée n'est pas si évidente : un nom peut être mal orthographié; de nombreuses sociétés ont plusieurs appellations (par exemple un acronyme, IBM, International Business Machines); certaines qualifications peuvent être ajoutées à un nom (Siemens, Siemens AG); le brevet peut être déposé par une société affiliée qu'il sera parfois facile d'identifier (comme Sony US, société affiliée à Sony), ou parfois difficile d'identifier (Citroën fait partie du groupe PSA). Il n'est pas inhabituel qu'un grand groupe ait une société affiliée, chargée de gérer sa propriété intellectuelle, et que cette société dépose à son nom un grand nombre de brevets pour le compte du groupe (c'est le cas de Philips, par exemple).

Les changements qui affectent le statut juridique de la société, les changements de raison sociale, d'affiliation, les fusions et les acquisitions, font que l'utilisation du nom du titulaire d'un brevet constitue un outil imparfait pour analyser l'activité des sociétés en matière de brevets et leur stratégie d'innovation. Par exemple, lorsque l'on s'efforce d'harmoniser les entités juridiques, tous les brevets détenus par Hewlett Packard, Digital Equipment Corporation et Compaq peuvent être considérés comme faisant partie d'une seule et même entité juridique; de même, les brevets de "Andersen Consulting " après harmonisation, seront attribués à " Accenture " (changement de raison sociale).

Les bureaux des brevets effectuent déjà un travail de nettoyage et d'harmonisation des informations. Par exemple, l'USPTO traite le nom du premier déposant de tout brevet. L'OEB attribue un code standard aux déposants, de même que le JPO pour les déposants par voie électronique. Mais cela ne suffit pas à répondre aux besoins des statisticiens. Le nettoyage et l'harmonisation des noms se fait en plusieurs étapes (lesquelles ne sont pas toutes nécessaires, ni exclusives les unes des autres) :

- Nettoyage de base (harmonisation des mots tels que « Ltd ", " GmbH », etc.) et standardisation des noms.

- Rattachement du nom standardisé des déposants aux noms contenus dans une base de données ou à une liste de référence sur les sociétés (par exemple, Amadeus pour l'Europe, Compustat pour les États-Unis).

- Reconstruction de la structure du groupe à l'aide d'informations sur la structure capitalistique (y compris les sociétés affiliées) trouvées dans les bases de données spécialisées (par exemple, la base « Who owns whom »).

La première étape consiste à identifier les différentes variantes orthographiques des noms des déposants, afin d'obtenir un nom standardisé permettant de regrouper les sociétés. On utilise pour cela des techniques 
d'association approximative. Deux méthodes sont notamment utilisées pour regrouper les noms similaires et standardiser les appellations. Les méthodes algorithmiques consistent à définir des règles pour établir et standardiser la similitude des noms ${ }^{10}$. La deuxième approche repose sur l'utilisation de dictionnaires, c'est-à-dire d'importantes collections de noms servant d'exemples pour une classe spécifique d'entités. On peut citer, en particulier, le fichier standard des associations de l'USPTO et de l'OEB; les codes Derwent de titulaires de brevets. Il est aussi possible de construire son propre dictionnaire en appliquant une procédure d'harmonisation (voir Magerman et al., 2006).

La deuxième étape consiste à associer les noms standardisés à des noms contenus dans une base de données de sociétés (par exemple, Amadeus, Compustat), soit directement, soit en combinaison avec d'autres méthodes, afin de trouver autant de correspondances que possible. On utilise les autres informations disponibles sur l'entreprise (outre sa raison sociale), comme son adresse, ainsi que les recherches faites à partir de noms apparentés de titulaires de brevets prioritaires ou des demandes PCT. Les correspondances ainsi obtenues doivent être validées, et les cas douteux ne peuvent être tranchés que par une intervention humaine. Enfin, les sociétés identifiées sont consolidées à l'aide d'informations sur leurs structures capitalistiques. Toutefois, ces deux étapes, la mise en correspondance et la consolidation statutaire, sont aussi réalisables simultanément si les données utilisées sur la société contiennent déjà des informations sur les relations entre les deux sociétés. Cependant, les données sur la structure capitalistique sont rarement harmonisées sur une longue période. On ne dispose par conséquent le plus souvent que de la structure la plus récente des sociétés. Aussi, il est nécessaire d'obtenir des informations complémentaires pour suivre ces évolutions (fusions et acquisitions) dans le temps et pour répartir correctement les activités de brevets entre sociétés à différentes périodes.

Dans ce domaine, il faut signaler le travail important réalisé par l'USPTO avec la base NBER, harmonisée avec Compustat (www.nber.org/patents), les algorithmes KUL pour Eurostat (Magerman et al., 2006) ainsi que les travaux de Thoma et Torrisi (2007) et Thoma et al. (à paraître).

\subsection{Brevets par inventeurs}

Identifier correctement les inventeurs, à partir des documents déposés, permet de retracer leur palmarès inventif et de compléter ces renseignements par des données provenant d'autres sources. Grâce aux informations harmonisées sur les noms des inventeurs, il est possible d'analyser un grand nombre de sujets intéressants et particulièrement pertinents au niveau politique. Par exemple :

- La productivité des inventeurs - sur le temps, répartition entre domaines, entre pays, etc. (Hoisl, 2007). 
- La mobilité des inventeurs - entre villes, régions, pays, secteurs (c'est-à-dire entre secteurs public et privé), et les retombées de cette mobilité (Kim et al., 2005; Crespi et al., 2005).

- Les stratégies de réseau des inventeurs - qui invente et avec qui? - et leur impact sur la productivité (Singh, 2003; Breschi et Lissoni, 2003).

- Les aspects hommes-femmes (répartition et profils sexués des inventeurs; voir Naldi et al., 2004).

Les avancées dans ce domaine ont été ralenties par les difficultés liées à l'enregistrement des noms dans les données de brevets et par la complexité de la tâche consistant à repérer " qui est qui " parmi les inventeurs figurant dans les données sur les brevets. Les informations concernant les inventeurs sont relativement peu exploitables en raison de trois problèmes fondamentaux. D'abord, le nom d'un même inventeur peut être orthographié de manière légèrement différente dans ses différents brevets (par exemple le deuxième prénom ou la deuxième initiale peuvent être absents, ou présents, de même que les annexes du nom de famille). Deuxièmement, même en présence de deux noms exactement similaires, il n'est pas toujours certain qu'il s'agisse d'une seule et même personne (le problème "John Smith "). En d'autres termes, des inventeurs différents portant exactement le même nom peuvent apparaître dans des brevets différents. Troisièmement, la transcription en alphabet latin de noms non occidentaux est imparfaite et peut être source d'ambigüités (" Li » ou « Lee »).

Des chercheurs ont entrepris d'harmoniser les noms à l'aide d'algorithmes d'association informatisés, appliqués jusqu'à présent à des sous-ensembles bien particuliers de données de brevets. Par exemple la méthode mise au point par Trajtenberg, Shiff et Melamed (2006), qui a été appliquée sur les données de brevets de l'USPTO, peut se résumer comme suit :

- Étape 1 : regroupement des noms similaires. Pour les cas où le nom d'un même inventeur est orthographié de manière légèrement différente sur plusieurs brevets, on adopte une approche en deux parties. La première consiste à " nettoyer » et standardiser les noms autant que possible; la seconde consiste à compléter la liste de noms harmonisés à l'aide du système "Soundex " pour encoder les noms qui se prononcent de la même manière ${ }^{11}$.

- Étape 2 : comparaison des noms et associations. Pour résoudre le problème qui consiste à identifier un individu spécifique parmi les "suspects " qui portent le même nom, les noms sont comparés et les critères d'association sont appliqués. Pour comparer des "suspects " deux à deux, on peut utiliser une série de variables comme le deuxième prénom, la situation géographique (code postal, ville, etc.), le domaine technologique (par exemple à partir des classes de brevet), l'attributaire, l'identité des co-inventeurs, etc. Si un champ est le même dans deux inscriptions "suspectes " (c'est-à-dire si deux enregistrements ont la même adresse ou appartiennent à la même 
classe de brevets ou ont les mêmes co-inventeurs, etc.) alors un certain score est affecté à la paire. Si la somme de ces scores est supérieure à un seuil prédéterminé, ces deux enregistrements sont " associés " et sont considérés comme correspondant au même inventeur ${ }^{12}$.

\section{Notes}

1. La CIB est structurée en sections, classes, sous-classes, groupes principaux et sous-groupes. La CIB divise les technologies brevetables en huit domaines clés ou sections (A : Nécessités courantes de la vie; B : Techniques industrielles diverses, transports $C$ : Chimie, métallurgie; D : Textiles, papier; $E$ : Constructions fixes; F : Mécanique, éclairage, chauffage, armement; G : Physique; $\mathrm{H}$ : Électricité). À l'intérieur de ces domaines, les technologies sont subdivisées jusqu'à un niveau de détail assez poussé, ce qui permet de classer avec une certaine précision le domaine d'une spécification de brevet.

2. Tous les documents japonais ne comportent pas de "F-terms »; leur présence dépend du domaine technologique.

3. Le code Y est un marqueur parallèle. Cela signifie qu'une application peut figurer dans n'importe quelle classe technologique CIB, mais si ses dimensions sont si réduites qu'elle peut être qualifiée de nano, elle reçoit le code Y. La définition des nanotechnologies de l'OEB est la suivante: Le terme de nanotechnologie se rapporte aux entités comportant au moins un composant fonctionnel dont la grosseur géométrique contrôlée est inférieure à $100 \mathrm{~nm}$ dans une ou plusieurs de ses dimensions, et qui est susceptible de produire un effet physique, chimique ou biologique du fait de cette dimension. Il couvre les équipements et méthodes permettant l'analyse contrôlée, la manipulation, le traitement, la fabrication ou la mesure avec une précision inférieure à $100 \mathrm{~nm}$.

4. L'indice ATR peut être appliqué, non seulement par rapport à la répartition sectorielle mondiale, mais aussi à d'autres groupes de référence (par exemple la répartition nationale ou régionale).

5. Pour construire la matrice de concordances, seuls les brevets d'une certaine importance ont été pris en compte. Ont été également considérés les entreprises du secteur manufacturier, le " principal " groupe de produits d'une société (bien que certaines grandes entreprises produisent une multitude de produits) ainsi que la première classe de la CIB.

6. Les adresses portées sur les brevets de l'OEB sont plus complètes que celles de l'USPTO et du PCT (WO) : dans la plupart des cas, le nom de la ville et les codes postaux figurent dans le champ adresse des brevets OEB, alors que dans les brevets USPTO, le code postal manque souvent et le processus de régionalisation repose essentiellement sur les noms de ville.

7. Les informations contenues dans la Base de données régionales sur les brevets (de l'OCDE) sont fondées sur la Base de données mondiale sur les statistiques des brevets de l'OEB (PATSTAT) : l'extraction de brevets déposés auprès de l'OEB, de l'USPTO et les inscriptions au PCT (publications WO); les inscriptions des inventeurs et des déposants proviennent des brevets OEB (données extraites du service en ligne OEBline).

8. Il convient de noter que les déposants individuels (particuliers) n'apparaissent pas comme une catégorie distincte dans la classification de Frascati; en outre, la 
catégorie "Étranger " n'a pas vraiment d'objet dans la classification des noms des détenteurs de brevets. Dans le Manuel de Frascati (2002) de l'OCDE, cinq secteurs sont identifiés : i) entreprises, ii) État, iii) secteur privé sans but lucratif, iv) enseignement supérieur, $v$ ) étranger. Les ménages sont considérés comme faisant partie du secteur privé sans but lucratif.

9. L'USPTO utilise une classification comprenant sept catégories: non affecté (s'applique aux brevets pour lesquels les inventeurs n'ont pas encore accordé les droits d'invention à une personne morale juridique); et affecté à : une organisation non gouvernementale des États-Unis, un individu de nationalité américaine, un individu de nationalité non américaine, le gouvernement fédéral des États-Unis, un gouvernement étranger.

10. On peut citer deux exemples : la « distance d'édition » de Levenshtein, qui mesure la similarité entre deux mots par le nombre minimal de caractères qu'il faut supprimer, insérer, ou remplacer pour passer d'un mot à l'autre; et la mesure de similarité de Jaccard, fondée sur le concept de jetons, qui compte les différences de position des mêmes jetons entre des chaînes de caractères identiques par ailleurs. D'autres algorithmes - notamment à base de jetons ou de n-grams utilisent souvent des indicateurs de type Jaccard pour la quantification finale de la similarité.

11. Soundex est un algorithme phonétique permettant d'indexer des noms sous forme de phonèmes - tels qu'ils sont prononcés en anglais. Le but est que les noms ayant la même prononciation soient représentés par un même encodage, de manière à pouvoir être associés malgré des différences orthographiques mineures.

12. Lorsque cette opération est réalisée pour toutes les paires de la série de comparaison, on applique la règle de la transitivité, c'est-à-dire que si un enregistrement $\mathrm{A}$ est associé à deux enregistrements $B$ et $C$, alors les trois brevets sont considérés comme relevant du même inventeur.

\section{Références}

Audretsch, D.B. et M.P. Feldman (1996), « R\&D Spillovers and the Geography of Innovation and Production ", American Economic Review, 86, pp. 630-640.

Breschi, S. et F. Lissoni (2001), «Knowledge Spillovers and Local Innovation Systems: A Critical Survey ", Industrial and Corporate Change, Oxford University Press, vol. 10(4), pp. 975-1005, décembre.

Breschi, S. et F. Lissoni (2003), « Mobility and Social Networks: Localised Knowledge Spillovers Revisited », CESPRI Working Papers 142, Centre for Research on Innovation and Internationalisation, Universita Bocconi, Milan.

Crespi, G.A., A. Geuna et L.J. Nesta (2005), « Labour Mobility of Academic Inventors: Career Decision and Knowledge Transfer ", SPRU Electronic Working Paper Series 139, University of Sussex, SPRU - Science and Technology Policy Research.

Dosi, G., K. Pavitt et L. Soete (1990), The Economics of Technical Change and International Trade, Harvester/Wheatsheaf.

Evenson, R.E., J. Putnam et S. Kortum (1991), « Estimating Patent Counts by Industry Using the Yale-Canada Concordance ", final report to the National Science Foundation.

Hoisl, K. (2007), "Tracing Mobile Inventors: The Causality between Inventor Mobility and Inventor Productivity ", Research Policy, 36, 619-636. 
Jaffe, A.B., M. Trajtenberg et R. Henderson (1993), "Geographic Localization of Knowledge Spillovers as Evidenced by Patent Citations ", Quarterly Journal of Economics 108, pp. 577-598.

Johnson, D. (2002), « The OECD Technology Concordance (OTC): Patents by Industry of Manufacture and Sector of Use ", OECD Science, Technology and Industry Working Paper 2002/5, Direction de la science, de la technologie et de l'industrie, OCDE, Paris, www.oecd.org/sti/working-papers.

Kim, J., S.J. Lee et G. Marschke (2005), « The Influence of University Research on Industrial Innovation », NBER Working Paper 11447, juin.

Magerman, T., B. Van Looy et X. Song (2006), « Data Production Methods for Harmonized Patent Statistics: Patentee Name Harmonization », KUL Working Paper $n^{\circ}$ MSI 0605.

Malerba F. et F. Montobbio (2003), « Exploring Factors Affecting International Technological Specialization: the Role of Knowledge Flows and the Structure of Innovative Activity ", Journal of Evolutionary Economics, vol. 13, nº 4, pp. 411-434.

Maraut, S., H. Dernis, C. Webb, V. Spiezia et D. Guellec (2008), " The OECD REGPAT Database: A Presentation ", OECD Science, Technology and Industry Working Paper 2008/2, Direction de la science, de la technologie et de l'industrie, OCDE, Paris, www.oecd.org/sti/working-papers.

Mowery, D.C., B.N. Sampat et A.A. Ziedonis (2001), « Learning to Patent: Institutional Experience, Learning, and the Characteristics of US University Patents after the Bayh-Dole Act, 1981-1992 ", Management Science 48(1), 73-89.

Naldi, F., D. Luzi, A. Valente et I.V. Parenti (2004), "Scientific and Technological Performance by Gender ", in H.F. Moed et al. (éd.), Handbook of Quantitative Science and Technology Research: The Use of Publication and Patent Statistics in Studies on R\&D Systems, Kluwer Academic Publishers, Dordrecht/Boston/Londres, pp. 299-314.

Noyons, E.C.M., R.K. Buter, A.F.J. van Raan, U. Schmoch, T. Heinze, S. Hinze et R. Rangnow (2003), « Mapping Excellence in Science and Technology across Europe. Nanoscience and Nanotechnology ", CWTS, Leiden.

OCDE (2002), Manuel de Frascati : Méthode type proposée pour les enquêtes sur la recherche et le développement expérimental, OCDE, Paris.

Paci, R. et S. Usai (2000), « Technological Enclaves and Industrial Districts: An Analysis of the Regional Distribution of Innovative Activity in Europe ", Regional Studies, Taylor and Francis Journals, vol. 34 (2), avril, pp. 97-114.

Pavitt, K. (1984), « Sectoral Patterns of Technical Change: Towards a Taxonomy and a Theory, Research Policy 13 (6) ", pp. 343-373.

Schmoch, U., F. Laville, P. Patel et R. Frietsch (2003), " Linking Technology Areas to Industrial Sectors ", final report to the European Commission, DG Research.

Singh, J. (2003), « Multinational Firms and Knowledge Diffusion: Evidence Using Patent Citation Data ", mimeo.

Soete, L. et S. Wyatt (1983), "The Use of Foreign Patenting as an Internationally Comparable Science and Technology Output Indicator", Scientometrics 5, January, pp. 31-54.

Thoma, G.L.D., S. Torrisi, A. Gambardella, D. Guellec, B.H.Hall et D. Harhoff (à paraître), "Harmonisation of Applicants' Names in Patent Data ", OECD Science, Technology and Industry Working Papers, Direction de la science, de la technologie et de l'industrie, OCDE, Paris, www.oecd.org/sti/working-papers. 
Thoma, G.L.D. et S. Torrisi (2007), « Creating Powerful Indicators for Innovation Studies with Approximate Matching Algorithms. A test based on PATSTAT and Amadeus databases ", CESPRI Working Papers 211, CESPRI, Centre for Research on Innovation and Internationalisation, Universita' Bocconi, Milan, Italie, révisé décembre, 2007.

Trajtenberg M., G. Shiff et R. Melamed (2006), « The 'Names Game': Harnessing Inventors' Patent Data for Economic Research », NBER Working Papers 12479, National Bureau of Economic Research, Inc.

Ulku, H. (2007), R\&D, «Innovation and Growth: Evidence from Four Manufacturing Sectors in OECD Countries ", Oxford Economic Papers, 59 (3), pp. 513-535.

Van Looy B., M. Du Plessis et T. Magerman (2006), « Data Production Methods for Harmonized Patent Statistics: Patentee Sector Allocation », Eurostat/K.U. Leuven Working Paper.

Veugelers, R. et B. Cassiman (2005), « R\&D Cooperation between Firms and Universities: Some Empirical Evidence from Belgian Manufacturing ", International Journal of Industrial Organization, 23, 5-6, pp. 355-379. 

Chapitre 6

\section{Utilisation et analyse des citations de brevets}




\subsection{Introduction}

Depuis une dizaine d'années, on utilise de plus en plus les citations de brevets et de littérature hors brevets comme indicateurs de l'innovation. Les citations étant un indicateur des précédents scientifiques et technologiques des inventions, leur étude permet de retracer l'évolution des connaissances. Il est possible d'identifier l'influence d'une invention particulière ou d'un ensemble d'inventions et de cartographier leur diffusion dans l'économie. En particulier, il est apparu que le nombre de citations dont un brevet faisait l'objet reflétait en moyenne l'importance technologique et commerciale de ce brevet, ce qui constitue une réponse au problème de l'hétérogénéité de la valeur des brevets.

Les citations permettent également d'étudier les relations entre les technologies, entre la science et la technologie, ou entre entreprises, branches, pays ou régions. Ces relations peuvent être ventilées de différentes manières : par domaine technologique, par type d'entité (entreprise multinationale ou nationale, université, etc.), par inventeur, etc.

Dans ce chapitre, nous décrivons la signification des citations de brevets et expliquons comment elles peuvent être utilisées pour compiler des indicateurs des sciences et des technologies. Nous examinons en particulier les problèmes qu'il faut prendre en considération lorsque l'on compile des indicateurs de brevets fondés sur les citations pour l'analyse de l'innovation. Ces principes directeurs peuvent servir de base à des progrès futurs dans ce domaine.

\subsection{Qu'est-ce qu'une citation?}

Les citations brevets et hors brevets sont les références contenues dans un rapport de recherche qui sont utilisées pour évaluer la brevetabilité d'une invention et permettent de juger de la légitimité des revendications d'une nouvelle application de brevet. Comme elles font référence à l'état antérieur de la technique, elles donnent des indications sur les connaissances préexistantes et peuvent aussi être invoquées pour démontrer le défaut de nouveauté de l'invention. Elles donnent aussi des indications sur les limites juridiques des revendications d'applications du brevet en question. Elles remplissent par conséquent une fonction juridique importante, en ce qu'elles déterminent la portée des droits de propriété couverts par le brevet. Si un brevet $B$ cite un brevet $A$, cela signifie que le brevet $A$ représente un élément 
d'un savoir préexistant, sur lequel le brevet B s'appuie ou auquel il se rapporte, et sur lequel le brevet $B$ ne peut avoir de revendication. Les citations peuvent donc aboutir à empêcher la délivrance d'un brevet ou à limiter la portée de la protection, qui ne doit pas recouper l'état antérieur de la technique au moment du dépôt de la demande.

Dans la plupart des cas, les citations sont le fruit d'une recherche approfondie sur l'état antérieur de la technique conduite par les examinateurs (sous forme d'un "rapport de recherche ") dont l'objet est d'apprécier le caractère de nouveauté et l'activité inventive d'une invention, nécessaires pour qu'elle soit brevetable. Les citations peuvent être utilisées pour rejeter les demandes de brevet lorsque l'invention revendiquée semble manquer de nouveauté après confrontation avec l'état antérieur de la technique. La recherche porte sur les documents scientifiques ou techniques disponibles publiquement ou sur tout autre témoignage qui constitue un précédent pertinent de cette invention.

On distingue deux grands types de citations. Les références aux brevets concernent les technologies antérieures pertinentes protégées ou décrites dans d'autres brevets déposés dans quelque pays, à quelque période et dans quelque langue que ce soit (voir tableau 6.2). Les références classées comme littérature hors brevets concernent les publications scientifiques, actes de conférences, ouvrages, supports de bases de données, manuels techniques, descriptions de standards, etc.

\subsection{Utilisations et applications des indicateurs de citations}

Les mesures s'appuyant sur les citations de brevets ont un potentiel considérable pour l'analyse des politiques. Trois applications des citations de brevets dominent la littérature consacrée à l'innovation : i) la mesure des flux de connaissances et des retombées (ex Jaffe et al., 1993); ii) la mesure de la qualité des brevets (par exemple, Harhoff et al., 2002); et iii) le comportement stratégique des sociétés (par exemple, Podolny et al., 1996).

Les citations en amont - c'est-à-dire les citations qui font référence à des documents de brevets antérieurs - sont utiles pour observer la diffusion des connaissances technologiques. Cette méthode permet d'estimer la courbe d'obsolescence des technologies, la diffusion de connaissances émanant d'inventions d'institutions, de zones ou de régions spécifiques, etc. Cela étant, les citations de brevets et de littérature hors brevets constituent parfois un piètre indicateur des flux de connaissances, car l'inventeur du brevet contenant la citation n'est pas toujours informé de l'existence du brevet cité, car les citations sont souvent ajoutées par l'examinateur ou le conseil en brevets (voir par exemple Jaffe et al., 2000) ${ }^{1}$. 
Les citations en aval - c'est-à-dire les citations contenues dans des brevets ultérieurs - peuvent être utilisées pour évaluer l'impact technologique des inventions, leurs retombées dans d'autres technologies ou dans d'autres régions géographiques. L'impact technologique des inventions peut être le reflet de l'importance économique des brevets. Une corrélation a été montrée à plusieurs reprises entre la valeur d'un brevet et le nombre et la qualité de ses citations en aval. Il est démontré qu'il existe une relation étroite entre les indicateurs pondérés en fonction du nombre de citations (par exemple du stock de brevets d'une entreprise) et les indicateurs économiques (valeur boursière des sociétés). Il apparaît également que les brevets qui reçoivent plus de citations que la moyenne ont aussi plus de chances d'être renouvelés (Lanjouw et al.,1998) et de faire l'objet de contestations ou d'actions en justice (par exemple, Lanjouw et Schankerman, 1997; Harhoff et al., 2002).

\subsection{Pratiques des bureaux des brevets en matière de citation}

Les pratiques en matière de citation diffèrent d'un bureau des brevets à l'autre : les indicateurs ne sont donc pas directement comparables. En raison des différences dans les obligations de publication et les procédures d'examen, les recherches menées pour les brevets européens sont assez différentes de celles pratiquées pour les brevets USPTO, de même que les citations générées dans les deux processus. Cela signifie que les chercheurs qui souhaitent recourir à l'analyse des citations doivent connaître ces différences.

Les déposants auprès de l'USPTO sont juridiquement tenus d'inclure une liste exhaustive de l'état de la technique connu ou supposé (" devoir de franchise »), liste qui est alors évaluée ou complétée par l'examinateur. Les examinateurs examinent la totalité de cette liste, à de rares exceptions près. Dans le système USPTO, il existe une forte motivation à fournir toutes les références à l'état antérieur de la technique, car à défaut, le déposant s'expose à des poursuites judiciaires et à de lourdes pénalités ${ }^{2}$.

À l'OEB, ces contraintes n'existent pas : la citation des inventions antérieures dans la demande par le déposant ou son conseil en brevets est facultative ${ }^{3}$. La plupart des citations figurant dans les publications PCT et OEB (soit environ $95 \%$ ) ont été ajoutées par les examinateurs dans le rapport de recherche. Si les examinateurs sont responsables de dresser la liste des références sur l'état antérieur de la technique (qui figure dans le rapport de recherche) par rapport à laquelle est établie la brevetabilité, ils s'appuient en partie sur les divulgations de l'état antérieur de la technique soumises avec la demande de brevet (par exemple à l'OEB, elles accompagnent l'exposé de l'invention).

De plus, le rapport de recherche OEB doit comprendre (en référence) les documents les plus importants, ou dans le cas de documents d'importance équivalente, les plus anciens. Dans la philosophie de l'OEB, un bon rapport de 
recherche doit renfermer au sein d'un nombre minimum de citations toutes les informations pertinentes ${ }^{4}$. D'aucuns ont noté que certains déposants à l'USPTO fournissent peut-être plus de références que nécessaire (jusqu'à la réforme de 2006). Ce fait, combiné à l'approche minimaliste des examinateurs de l'OEB, explique en grande partie pourquoi le nombre moyen de citations dans les brevets de l'USPTO est considérablement plus élevé que dans les brevets OEB (tableau 6.1) ${ }^{5}$.

\section{Tableau 6.1. Occurrence des références brevets et hors brevets (USPTO - OEB)}

\begin{tabular}{|c|c|c|c|c|c|}
\hline \multicolumn{6}{|c|}{ Brevets délivrés par l'USPTO - dépôt entre 1991 et 2001} \\
\hline Nombre total de brevets (1) & $\begin{array}{l}1299 \\
817\end{array}$ & $\begin{array}{l}\text { Nombre total } \\
\text { de références }\end{array}$ & $\begin{array}{l}17757 \\
797\end{array}$ & & \\
\hline $\begin{array}{l}\text { Nombre de brevets contenant } \\
\text { des références à des brevets }\end{array}$ & $\begin{array}{l}1173 \\
593 \\
(90 \%)\end{array}$ & $\begin{array}{l}\text { Nombre de références } \\
\text { à des brevets }\end{array}$ & $\begin{array}{l}14738 \\
854 \\
(83 \%)\end{array}$ & $\begin{array}{l}\text { Intensité technologique. } \\
\text { Dénominateur (1): }\end{array}$ & $\begin{array}{l}12.55 \\
11.33\end{array}$ \\
\hline $\begin{array}{l}\text { Nombre de brevets contenant } \\
\text { des références hors brevets }\end{array}$ & $\begin{array}{l}445466 \\
(34 \%)\end{array}$ & $\begin{array}{l}\text { Nombre références } \\
\text { hors brevets }\end{array}$ & $\begin{array}{l}3018 \\
943 \\
(17 \%)\end{array}$ & $\begin{array}{l}\text { Intensité de références hors } \\
\text { brevets. Dénominateur (1): }\end{array}$ & $\begin{array}{l}6.77 \\
2.2\end{array}$ \\
\hline \multicolumn{6}{|c|}{ Brevets délivrés par l'OEB - dépôt entre 1991 et 2001} \\
\hline Nombre total de brevets (1) & 342704 & $\begin{array}{l}\text { Nombre total } \\
\text { de références }\end{array}$ & $\begin{array}{l}1698 \\
218\end{array}$ & & \\
\hline $\begin{array}{l}\text { Nombre de brevets contenant } \\
\text { des références à des brevets }\end{array}$ & $\begin{array}{l}334413 \\
(98 \%)\end{array}$ & $\begin{array}{l}\text { Nombre de références } \\
\text { à des brevets }\end{array}$ & $\begin{array}{l}1404 \\
241 \\
(83 \%)\end{array}$ & $\begin{array}{l}\text { Intensité technologique. } \\
\text { Dénominateur (1): }\end{array}$ & $\begin{array}{l}4.20 \\
4.09\end{array}$ \\
\hline $\begin{array}{l}\text { Nombre de brevets contenant } \\
\text { des références hors brevets }\end{array}$ & $\begin{array}{l}130511 \\
(38 \%)\end{array}$ & $\begin{array}{l}\text { Nombre de références } \\
\text { hors brevets }\end{array}$ & $\begin{array}{l}293977 \\
(17 \%)\end{array}$ & $\begin{array}{l}\text { Intensité de références hors } \\
\text { brevets. Dénominateur (1): }\end{array}$ & $\begin{array}{l}2.25 \\
0.86\end{array}$ \\
\hline
\end{tabular}

Source : Callaert et al. (2006).

$\mathrm{Au}$ JPO, ce sont les examinateurs de brevets qui sont chargés des recherches sur l'état antérieur de la technique; toutefois, les déposants sont aussi tenus de fournir préalablement des informations sur l'état antérieur de la technique (en pratique depuis Septembre 2002 et de manière obligatoire depuis mai 2006). Le nombre de références fournies n'est pas plafonné.

S'agissant des citations OEB et PCT, il convient de prêter attention aux problèmes suivants (Webb et al., 2005) :

- Les citations contenues dans les rapports de recherche internationaux et régionaux peuvent varier. L'un des problèmes est lié au caractère (partiel) et substitutif des informations contenues dans les rapports de recherche WO (le Rapport de recherche internationale) ${ }^{6}$. Si l'OEB reçoit des dépôts qui ont été préalablement traités par d'autres ISA (autorités chargées de la recherche internationale), l'OEB va entreprendre une recherche supplémentaire, dont la synthèse sera présentée dans le Rapport de recherche complémentaire ${ }^{7}$. 
- Ce phénomène est particulièrement important sachant qu'un nombre croissant de déposants commencent par la procédure PCT avant d'engager le processus auprès de l'OEB dans la "phase régionale ". Dans ce cas, la plupart des citations apparaîtront dans le document international (WO) et non dans le document OEB (dénoté EP). Pour un juste comptage des citations, il faut combiner les informations de la recherche internationale et de la recherche européenne.

Jusqu'à une date récente, la plupart des indicateurs de citations étaient limités à un seul bureau : pour les brevets OEB, uniquement les références à des brevets OEB antérieurs, et pour les brevets USPTO, uniquement les références à des brevets USPTO antérieurs. Pour les brevets OEB, il a été démontré qu'environ trois quarts des références ne sont pas utilisées. La prise en compte de la totalité des données aurait un impact considérable sur les indicateurs de citations. Par exemple, l'inclusion des citations en phase internationale selon le PCT (WO) avec les équivalents OEB change considérablement la donne s'agissant du délai de citation (temps écoulé entre brevets cités et brevets " citant "); le délai médian passe de 4.0 à 6.7 ans; le délai maximum passe de 25.7 à 132 ans (Harhoff et al., 2006).

Plusieurs problèmes doivent être pris en considération lorsque l'on travaille avec les citations de brevets et de littérature hors brevets. Quelquesuns des plus importants pour le comptage des citations sont mentionnés ici.

Il n'existe pas une relation bijective entre les documents de brevets et les inventions. Les citations d'un brevet peuvent prendre différentes formes. Une même invention peut être couverte par plusieurs documents émis par différents bureaux nationaux et supranationaux (Harhoff et al., 2006) ${ }^{8}$. Un brevet peut être cité comme étant une publication de brevet nationale, internationale ou régionale, ou comme provenant de l'USPTO, de l'OEB ou du JPO. Comme on l'a vu au chapitre 4, tous les dépôts effectués dans différents pays, ainsi que les brevets accordés par la suite sur une invention, sont généralement considérés comme brevets équivalents. Un groupe de brevets équivalents forme une famille de brevets, c'est-à-dire un ensemble de brevets (ou de demandes de brevets) déposés dans plusieurs pays pour protéger une même invention. Ils sont liés entre eux par un ou plusieurs numéros de priorité communs. Lorsque ces différentes citations ne sont pas prises en compte, le comptage des citations est sous-estimé parce que les citations d'une invention donnée sont réparties entre les différentes versions de la famille de brevets.

À l'OEB, le référencement s'efforce de retenir la manifestation la plus ancienne et la plus facilement accessible d'une invention, de préférence dans la langue du déposant. Dans les documents OEB, la majorité (environ les trois quarts) des références renvoient à des documents non OEB. À cet égard, 


\section{Encadré 6.1. Le problème des brevets équivalents}

\section{Le cas des citations de brevets européens (Harhoff et al., 2006)}

Le comptage des citations de brevets européens est très souvent utilisé en analyse économique mais peu d'études ont traité le problème des équivalents (un brevet qui se rapporte à une même invention et partage la même demande prioritaire qu'un brevet émis par une autre autorité; voir au chapitre 4 la définition des familles de brevets). Le schéma ci-dessous montre la structure de ce problème. Le rapport de recherche pour la demande de brevet EP-x cite en référence les documents de brevet WO-a, EP-w et US-z. Toutefois, le document WO-a possède un équivalent EP-y parmi les demandes déposées auprès de l'OEB. La demande de brevet qui correspond au document US- $z$ possède deux équivalents (EP-z et EP-v) dans le système EP.

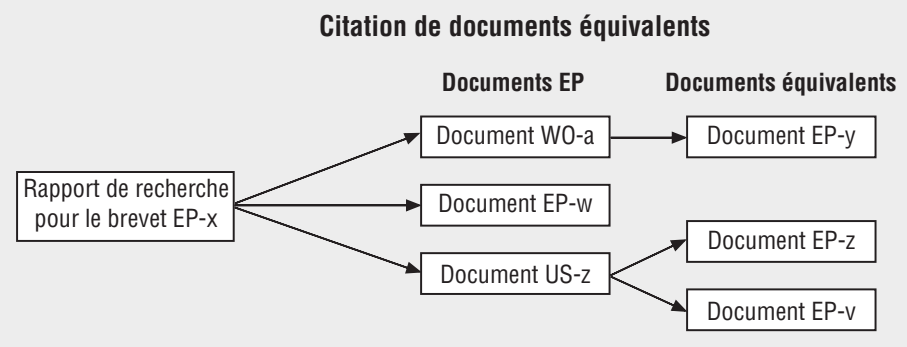

Ce système de référencement n'est nullement défaillant. Il est en fait fréquemment observé, étant donné les contraintes de délais, les goulets d'étranglement des systèmes de documentation ou tout simplement les préférences linguistiques. Toutefois, pour un chercheur qui souhaite savoir combien de fois des brevets particuliers (par exemple EP-y, EP-z ou EP-x) ont été cités sous une forme ou sous une autre (équivalente), le simple comptage du nombre d'occurrences sans appliquer de correction est un mauvais indicateur. Pour obtenir un juste comptage des citations, il conviendrait, pour tous les documents non-EP, de reprendre le(s) numéro(s) de demandes de l'équivalent EP avant de procéder au comptage.

Pour être plus précis, la règle à appliquer peut être exprimée comme suit : Soient $\mathrm{X}$ et $\mathrm{Z}$ deux bureaux des brevets différents. Toute référence à un document de brevet dans le système $\mathrm{X}$ doit être comptabilisée comme une citation valide d'un brevet particulier dans le système $Z$ si le document du système $X$ est un équivalent $d u$ brevet du système $Z$. Dans un nombre non négligeable de cas, le document non-EP auquel il est fait référence est lié à plus d'un équivalent EP, comme l'illustre le graphique ci-dessus. Le comptage fractionnaire peut alors être appliqué - c'est-à-dire que le comptage des citations et les autres statistiques sera pondéré en fonction de l'incidence (ou donnée statistique) de chacun des équivalents EP par l'inverse du nombre d'équivalents EP. 
Michel et Bettels (2001) montrent que $90 \%$ du total des citations de brevets faites par l'OEB renvoient à des documents EP (OEB), DE (Deutsches Patentund markenamt - DPMA), GB (United Kingdom Intellectual Property Office), WO (OMPI), ou US (USPTO). Dans ce contexte, le comptage de citations réalisé uniquement sur les documents sont sous-estimés. Dans le cas des citations de brevets US, le problème existe aussi, mais à un moindre degré, car l'USPTO cite essentiellement les documents USPTO. À l'USPTO et au JPO, $90 \%$ ou plus des références contenues dans les rapports de recherche font référence à des documents nationaux (Michel et Bettels, 2001).

\subsection{Les indicateurs fondés sur les citations}

\subsubsection{Comparaison des citations}

Les informations qui reposent sur les citations de brevets n'ont de sens que comparativement. Il n'existe pas une échelle absolue ou une mesure de valeur associée aux chiffres de citations. Pris isolément, le fait qu'un brevet donné ait fait l'objet de 10 ou de 100 citations ne dit pas si ce brevet est "beaucoup " cité. En d'autres termes, l'évaluation de l'intensité de citations d'une invention, d'un inventeur, d'une institution ou d'un groupe de référence, ne peut être faite que par rapport à une référence d'intensité de citation.

En principe, il est possible de déceler et de quantifier les évolutions de l'intensité de citations associées aux différents effets. Toutefois, il n'est pas évident de savoir si les phénomènes observés sont réels ou artificiels : il y a par conséquent des risques de mauvaise interprétation des indicateurs. Prenons par exemple certains des faits stylisés observés concernant les chiffres des citations des brevets USPTO : i) le nombre moyen de citations reçues par les brevets dans leurs cinq premières années s'accroît avec le temps; ii) le nombre moyen de citations par brevet s'accroît avec le temps; et iii) les profils de délais de citations pour les cohortes les plus anciennes ont des "queues " plus épaisses que ceux des cohortes plus récentes.

Le premier de ces faits stylisés, pris isolément, pourrait amener à conclure, soit que les cohortes de brevets plus récentes sont plus "fertiles ", soit que la distribution des délais de citations s'est décalée vers la gauche (c'est-à-dire que les citations interviennent plus tôt que par le passé). Le deuxième pourrait conduire à croire qu'il y a eu un changement artefactuel dans la propension à citer. Mais comme le stock de brevets disponibles et susceptibles d'être cités a progressé à un rythme rapide (voire de plus en plus rapide) cela n'est pas évident. Le dernier fait, pris isolément, semble suggérer que la distribution du délai de citation s'est déplacée sur la droite. Sans autres hypothèses, on ne peut pas dire lequel de ces scenarios mutuellement exclusifs est le bon, et on ne peut par conséquent pas apporter d'ajustements statistiques aux chiffres de citations, notamment pour la troncature des citations sur toute la durée de vie des brevets. 
La détermination du repère approprié est compliquée par plusieurs phénomènes propres aux données sur les citations de brevets (Hall et al., 2001).

- D'abord, le nombre de citations reçues par un brevet donné est tronqué dans le temps, puisqu'on ne connaît que le nombre de citations reçues jusqu'à maintenant. Plus important, des brevets d'âges différents subissent différents degrés de troncature. Les brevets plus récents ont nécessairement eu moins de temps pour être cités.

- Deuxièmement, les changements qui interviennent dans les pratiques d'examen des brevets peuvent entraîner des écarts dans l'intensité de citation qui ne sont pas liés à l'impact réel que l'on souhaite mesurer en utilisant les citations comme données de substitution. Dans les données NBER USPTO sur les citations de brevets, le brevet moyen émis en 1999 contient plus de deux fois plus de citations que le brevet moyen délivré en 1975 (10.7 contre 4.7).

- Troisièmement, le problème créé par l'accroissement du nombre de citations par brevet est exacerbé par le fait que le nombre de brevets émis a lui aussi connu une forte augmentation dans plusieurs bureaux des brevets. Même si chaque brevet émis contenait le même nombre de citations que par le passé, la multiplication des brevets « citant » augmenterait le nombre total de citations faites. Les augmentations conjuguées du nombre de brevets et $d u$ nombre de citations par brevet suggèrent une certaine forme d'« inflation " des citations, qui signifie que les citations récentes ont moins de poids d'un point de vue statistique que les anciennes.

- Enfin, le nombre de citations par brevet (en amont et en aval) varie considérablement en fonction du domaine technologique ou de la maturité de la technologie. En général, les brevets des domaines technologiques traditionnels citent plus qu'ils ne sont cités, alors que les brevets des domaines émergents tels que l'informatique et les communications, les médicaments et les technologies médicales, sont beaucoup plus cités mais citent relativement moins. Le degré de dépendance à l'égard des technologies antérieures ou la " cumulativité " détermine la propension à citer d'autres brevets; par exemple, les technologies telles que les semi-conducteurs ont généralement une forte intensité de citations en amont ${ }^{9}$.

Deux approches génériques sont employées pour remédier à ces problèmes. La première, l'approche des effets fixes consiste à harmoniser le nombre de citations en les divisant par le nombre moyen de citations pour le groupe de brevets auquel appartient le brevet examiné. Cette approche suppose que toutes les sources de variation systématique dans le temps de l'intensité de citation sont un artefact dont il faut corriger les effets avant de comparer l'intensité de citation des brevets de cohortes différentes. En d'autres termes, l'intensité de citation est recalculée, exprimée sous forme de ratio par rapport à l'intensité de citation moyenne des brevets de la même cohorte. 
Pour comparer un brevet de 1990, avec deux citations, avec un brevet de 1985 , avec quatre citations, on divise chaque valeur par le nombre moyen de citations reçues par l'ensemble des brevets de la même cohorte. Cela permet de purger les données de l'impact de la troncature, des effets liés à d'éventuels changements systématiques dans le temps de la propension à citer, et des effets liés au changement du nombre de brevets contenant des citations. Malheureusement, cela purge aussi les données des mouvements systématiques dans le temps en ce qui concerne l'importance ou l'impact de certaines cohortes de brevets. L'avantage de cette approche est qu'elle ne nécessite pas de formuler des hypothèses quant aux processus sous-jacents susceptibles d'induire des différences d'un groupe à l'autre dans l'intensité de citation. L'inconvénient est que, comme justement on ne suppose aucune structure, elle ne distingue pas entre les différences "réelles » et celles qui sont susceptibles d'être des artefacts.

La seconde approche, dite quasi-structurelle consiste à dégager les différents phénomènes qui influent sur le taux de citation au moyen d'une estimation économétrique. Une fois les différents effets ainsi quantifiés, le chercheur a la possibilité d'opérer un ajustement sur le comptage " brut » des citations afin d'éliminer un ou plusieurs des effets estimés. Si les hypothèses qui soustendent l'estimation économétrique sont justes, cette approche permet d'extraire un "signal " plus fort à partir des données "bruitées » du nombre de citations, que ne le permet l'approche non structurelle des effets fixes (voir Hall et al., 2001, pour en savoir plus sur la méthode d'estimation).

\subsubsection{Indicateurs fondés sur les citations en amont}

Deux groupes d'indicateurs peuvent être construits avec les citations. Le premier concerne les indicateurs fondés sur les citations en amont, qui sont utiles pour évaluer le degré d'innovation de l'invention et observer les voies par lesquelles s'opèrent les transferts de connaissances (par exemple les réseaux de citations). Le second groupe rassemble les indicateurs d'impact, basés sur les citations en aval. Par ailleurs, on peut construire des mesures basées sur les citations qui peuvent rendre compte d'autres aspects des inventions brevetées : " originalité ", " généralité », « base scientifique » (voir par exemple Trajtenberg et al., 1997; Narin et al., 1997; Sampat et Ziedonis, 2004).

La cumulativité technologique se définit par la fréquence d'autocitation des brevets qui sont le fruit de recherches antérieures de la même société. La mise en évidence des autocitations (du déposant ou du titulaire) a des implications importantes, notamment pour l'étude des retombées technologiques : on peut penser que les citations de brevets appartenant au même titulaire représentent des transferts de connaissances qui sont essentiellement internalisées, alors que les citations de brevets d'autres personnes correspondent davantage à la notion pure de retombées (diffusion). Il est plus approprié d'exclure les autocitations 
(lorsque l'on dispose d'informations consolidées sur les données de brevets) lorsque l'on étudie les transferts de connaissances ou l'impact des inventions en termes de citations.

Une mesure commune de la cumulativité au niveau de l'entreprise est la somme des citations en amont à des brevets possédés par celle-ci, divisée par le nombre total de ses brevets (à un temps $t$ donné). D'après Malerba et Orsenigo (1995), la cumulativité suppose que les inventeurs novateurs aient un avantage sur les « suivistes » et que les premiers continueront probablement de donner le «la » à l'avenir.

Délai de citation : Le terme de "délai de citation " est la mesure du temps écoulé entre une date caractéristique du brevet "citant» et une date caractéristique du document cité. Le délai est donc l'écart temporel entre l'année du dépôt, de la publication ou de la délivrance du brevet citant, et celle des brevets cités. Les délais de citation peuvent être calculés de diverses manières : ils peuvent se baser sur la date de priorité, sur la date de dépôt ou sur la date de publication. Par ailleurs, il y a deux moyens de considérer les délais de citations : en aval et en amont. La mesure du délai calculée dans la série de données sur les citations de l'OCDE sur l'OEB est définie comme la durée écoulée entre la publication du dépôt du brevet cité (en général, les brevets et la littérature hors brevets ne peuvent pas être cités avant d'être publiés, sauf s'il s'agit d'une invention revendiquée par le même déposant) et la date de publication du rapport de recherche du brevet citant (Webb et al., 2005). Soulignons quelques implications qui découlent de ce choix :

- Pour la plupart des documents de brevets cités provenant de bureaux des brevets européens ou du JPO, la publication (notamment la divulgation des résultats de la recherche au public dans le cas de l'OEB) intervient exactement 18 mois après la date de priorité. Par conséquent, pour le calcul des délais de citation des brevets européens ou japonais, peu importe que l'on choisisse la date du rapport de recherche (la date de priorité pour les brevets japonais) ou la date de publication du dépôt. On peut prendre pour référence la date de priorité du brevet citant avec la date de publication du brevet cité.

- Si le document cité est un brevet américain pour lequel la demande de brevet n'a été faite qu'aux États-Unis, la première date de publication était la date de délivrance jusqu'en novembre 2000, et les déposants ont toujours la possibilité de s'en tenir à cette règle s'ils le souhaitent. Si le brevet américain cité possède un équivalent international, les demandes internationales correspondantes sont, là aussi, publiées 18 mois après la date de priorité aux États-Unis ${ }^{10}$.

- S'agissant des documents de brevets pour lesquels un rapport de recherche international a été publié par l'OMPI et un rapport complémentaire a été 
publié par l'OEB ou un autre ISA, on a plusieurs dates de publication. Si les documents ne sont nullement simultanés, le délai peut être calculé à partir de la date de publication du rapport de recherche pertinent. Si le rapport international de recherche et le rapport complémentaire de l'OEB citent le même document, on peut ignorer la dernière citation et prendre en compte la première date de publication des deux rapports de recherche pour le calcul du délai de citation.

Durée du cycle technologique (TCT) : À partir de la mesure des délais de citation, on peut calculer un indicateur au niveau de l'entreprise. La durée du cycle technologique indique la vitesse de l'innovation ou la vitesse à laquelle la technologie se renouvelle, définie par l'âge médian en nombre d'années des brevets cités en première page des brevets d'une société. Une société dont les cycles sont plus courts que ses concurrentes est une société qui passe plus vite d'une technologie à la suivante. Dans les semi-conducteurs, les cycles tendent à être courts (trois à quatre ans); dans la construction navale ils sont longs (plus de dix ans). La moyenne est de huit ans.

\subsubsection{Indicateurs reposant sur les citations en aval}

Nombre de citations en aval par brevet : on considère qu'il mesure l'impact technologique des inventions. Plusieurs études ont montré que le nombre de citations dont fait l'objet un brevet est associé à son importance technologique et sociale (Trajtenberg, 1990; Scherer et al., 1999) et qu'il est corrélé avec le renouvellement du brevet, la valeur économique estimée des inventions et la probabilité de contestation du brevet ou de litige (Lanjouw et Schankerman, 1999; Harhoff et al., 2002).

L'impact de citation est le nombre de citations en aval, mais exprimé en termes relatifs (voir à la section 6.5.1 les inconvénients de cette approche lorsque l'on compare les indicateurs dans le temps). Il s'agit du nombre de fois qu'un brevet est cité par rapport au nombre moyen de citations reçues par un brevet du même domaine technologique (sous-classe CIB à trois chiffres) et de la même date d'invention (année de priorité). Cette approche permet de contrôler les différences de fréquence de citation d'un domaine technologique à l'autre et l'effet de troncature lié au temps (les brevets plus anciens ont intrinsèquement une plus faible probabilité d'être cités; voir Hall et al., 2001).

Le degré de généralité d'un brevet se construit comme un indice de Herfindahl (Trajtenberg et al., 1997; Hall et al., 2001) : Généralité $=1-\sum_{i}^{n i} s_{i j}{ }^{2}$, où $s_{i j}$ représente le pourcentage de citations reçues par un brevet $i$ appartenant à la classe $j$, sur $n_{i}$ classes de brevets ${ }^{11}$. Un niveau de généralité élevé suggère que le brevet a un large impact, puisqu'il influence les innovations ultérieures dans différents domaines. L'impact géographique d'un brevet peut se construire de manière similaire (1-indice Herfindahl de concentration géographique), c'est-à-dire dans les différents pays d'origine des inventeurs qui citent le 
brevet. Le degré d'originalité d'un brevet peut être défini de la même manière, sauf qu'il se base sur les citations en amont. Ainsi, si un brevet cite des brevets antérieurs appartenant à un ensemble étroit de technologies, son score d'originalité sera faible, alors qu'un brevet qui puise ses citations dans un grand nombre de domaines recevra un score élevé.

Il convient de prendre en compte un certain nombre d'éléments lors du calcul d'indicateurs de ce type :

- Les mesures d'originalité et de généralité dépendent du système de classification des brevets : une classification plus fine produira des mesures plus élevées et un système plus grossier des mesures inférieures. Par conséquent, si la classification à l'intérieur d'un domaine est plus fine (en nombre de classes de brevets à trois chiffres), on aboutira, toutes choses étant égales par ailleurs, à de plus forts scores d'originalité et de généralité, ce que l'on peut considérer comme un artefact du système de classification.

- Comme le montrent Hall et al. (2001), la mesure de généralité est biaisée vers le haut lorsque le nombre de brevets sur lesquels elle est calculée est faible. S'il existe une "vraie » probabilité qu'un brevet pris au hasard appartienne à une classe parmi un grand nombre, la concentration réelle peut être faible: si l'on n'observe en réalité qu'un très petit nombre de brevets, ils ne peuvent appartenir qu'à un petit nombre de classes, et la concentration mesurée sera élevée. Il convient d'ajuster l'indicateur en fonction du nombre d'observations ${ }^{12}$.

$\mathrm{Au}$ niveau de l'entreprise, plusieurs indicateurs sont utilisés pour mesurer l'impact des brevets (Narin, 2000) :

- L'indice de l'incidence actuelle (IIA) : Le nombre de fois que les brevets déposés par une société dans les cinq années précédentes sont citées dans l'année courante, par rapport à l'ensemble des brevets du système américain, dénote la qualité du portefeuille de brevets. Une valeur de 1.0 représente une fréquence de citation moyenne; une valeur de 2.0 représente une fréquence de citation deux fois plus élevée; et un indice de 0.25 représente $25 \%$ de la fréquence moyenne de citation. Cela permet de comparer la qualité technologique d'une société par rapport à d'autres sociétés et par rapport à l, valeur qui représente la moyenne pour la technologie. Cet indice varie selon les domaines technologiques. Par exemple dans les domaines des semi-conducteurs, des biotechnologies et des produits pharmaceutiques, il est élevé, alors que dans le verre, l'argile et le ciment et le textile, il est faible. Il est apparu que cet indice avait une corrélation avec la performance boursière d'une société.

- «Force technologique » (FT) : C'est la taille du portefeuille de brevets pondérée en fonction de leur qualité, définie comme le nombre de brevets multiplié par l'indice d'incidence actuelle. Grâce à la FT, on peut constater qu'une 
société est technologiquement plus forte qu'une société concurrente détenant pourtant davantage de brevets si ses brevets sont de meilleure qualité. Une société dont les brevets sont très cités peut être plus avancée que ses concurrentes et avoir un portefeuille de brevets de plus grande valeur.

- L'indice de performance de citation : C'est le calcul d'un indice relatif exprimant le nombre de brevets comptant parmi les plus souvent cités (par exemple les $10 \%$ ) détenus par un pays particulier (ou tout autre entité) par rapport au reste du monde (ou tout autre référence pertinente). Cet indicateur mesure également l'impact de la qualité des brevets d'un groupe de référence. Pour un pays, la formule de l'indicateur est égale au pourcentage des brevets d'un pays i figurant parmi les 10 \% plus cités par rapport au même pourcentage pour la totalité des brevets au niveau mondial.

\subsection{Littérature hors brevets (NPL)}

Les indicateurs de lien scientifique sont fondés sur le nombre de références à la littérature hors brevets considérée comme scientifique. La distinction des références d'ordre scientifique permet d'isoler les technologies qui sont plus proches de la R-D scientifique, et donc plus dépendantes du progrès des connaissances scientifiques. Ils reconnaissent le fait que les références à la littérature hors brevets sont utiles pour examiner l'interaction entre la science et la technologie. Le nombre moyen de références hors brevets est souvent utilisé comme variable de substitution pour quantifier la relation d'un domaine technologique avec une discipline scientifique (Narin et al., 1997; Meyer, 2000; Verbeeck et al., 2002). Plus on trouve de références scientifiques dans les brevets, plus la technologie est considérée comme proche de la recherche fondamentale. L'analyse des liens des brevets avec la science peut être étendue à des thèmes importants pour les politiques, notamment l'influence de la science sur les domaines technologiques émergents, ou la valeur de la science pour l'industrie (son impact sur la valeur économique des entreprises).

Toutefois, les références hors brevets doivent être utilisées avec précaution et il convient de prendre en considération certains éléments propres au contexte lorsque l'on interprète ces indicateurs. Comme on l'a vu à la section 6.4, les différences entre bureaux des brevets au niveau des procédures d'examen peuvent avoir un impact sur le nombre et le type de références citées. Dans le cas de l'OEB, comme les références sont, pour la plupart, insérées par l'examinateur après qu'il a passé en revue l'état de la technique, certains ont pu observer que les citations sont rarement le reflet des connaissances scientifiques utilisées par les inventeurs. D'autres chercheurs indiquent que les références hors brevets ne représentent qu'un lien unidirectionnel avec la science et qu'il est difficile d'établir la relation de cause à effet entre les deux documents, le brevet contenant la citation et l'article cité (Tijssen, 2002). 
La littérature hors brevets se compose non seulement des communications scientifiques revues par les pairs, mais aussi d'autres types de publications : actes de conférences, bases de données (structures d'ADN, séquences de gènes, composés chimiques, etc.) et d'autres documents pertinents (guides de traduction, manuels statistiques, etc.). Le tableau 6.2 montre l'occurrence des revues scientifiques et des autres sources dans les brevets USPTO et OEB et le tableau 6.3 rend compte des différentes catégories d'autres sources. Parmi les autres sources, les actes de conférences, la documentation technique et les bases de données sont les plus fréquemment cités. Les références aux documents non scientifiques tels que les abrégés de brevets et les services commerciaux de bases de données sur les brevets doivent être exclues de l'analyse des liens des brevets avec la science.

D'après une analyse menée sur de plus de 540000 demandes internationales de brevets (déposés selon le traité de coopération en matière de brevets, PCT) publiées par l'OEB ces 15 dernières années, il apparaît que les sous-classes CIB caractérisées par un nombre de citations de NPL plus élevé que la moyenne (plus de $15 \%$ ) appartiennent en majorité aux domaines des

Tableau 6.2. Occurrence des références aux revues scientifiques et aux autres sources dans les brevets USPTO et OEB

Valeurs observées (pourcentages de la rangée entre parenthèses)

\begin{tabular}{lccc}
\hline & Revues scientifiques & Autres sources & Total références hors brevets \\
\hline USPT0 & $2766(55 \%)$ & $2242(45 \%)$ & 5008 \\
OEB & $3218(64 \%)$ & $1803(36 \%)$ & 5021 \\
Total & 5984 & 4045 & 10029 \\
\hline
\end{tabular}

Source : Callaert et al. (2006).

Tableau 6.3. Occurrence des sources autres que les revues scientifiques dans les citations de brevets USPTO et OEB

Valeurs constatées (pourcentages de la colonne entre parenthèses)

\begin{tabular}{lrrr}
\hline & \multicolumn{1}{c}{ USPTO } & OEB & Total \\
\hline Actes de conférences & $381(17 \%)$ & $612(34 \%)$ & 993 \\
Documentation industrielle & $560(25 \%)$ & $304(17 \%)$ & 864 \\
Livres & $333(15 \%)$ & $186(10 \%)$ & 519 \\
Ouvrages et bases de données de référence & $234(10 \%)$ & $600(33 \%)$ & 834 \\
Documents liés aux brevets & $327(15 \%)$ & $46(3 \%)$ & 373 \\
Rapports de recherche et techniques & $138(6 \%)$ & $27(2 \%)$ & 165 \\
Journaux & $106(5 \%)$ & $10(0 \%)$ & 116 \\
Inconnu/autres & $163(7 \%)$ & $18(1 \%)$ & 181 \\
Total & $2242(100 \%)$ & $1803(100 \%)$ & 4045 \\
\hline
\end{tabular}

Source: Callaert et al. (2006). 
biotechnologies, des médicaments, des autres formes de chimie fine organique et des TIC (graphique 6.1) ${ }^{13}$. Le nombre de citations de littérature hors brevets tend à être plus élevé dans les pays dont l'activité de brevet internationale est plus concentrée sur ces domaines à forte activité de brevetage ou de technologies émergentes (graphique 6.2). Par exemple, les inventeurs indiens ont des antécédents récents d'activité internationale de brevetage et une proportion relativement élevée de leurs applications appartient aux domaines des biotechnologies et des produits pharmaceutiques, qui présentent des liens plus étroits avec la science.

Dans la période 1990-2004, environ 55 \% des citations contenues dans les brevets liés aux biotechnologies font référence à la littérature hors brevets. On n'observe guère de variations d'un pays à l'autre, ce qui semble indiquer qu'il existe une certaine homogénéité dans le rythme des avances technologiques, mais qui cache toutefois certaines différences structurelles entre pays. Pour les TIC (graphique 6.3), la proportion moyenne est d'environ $18 \%$ et se situe selon les pays entre 10 et $25 \%$. Lorsque cette proportion est faible, cela indique que les innovations de TIC récentes tendent à s'appuyer sur les technologies existantes; lorsqu'elle est élevée, cela suggère que le pays continue de tirer parti de la R-D scientifique dans les TIC.

Une fois identifiées les références à la littérature hors brevets à caractère scientifique, on peut dégager plus clairement l'influence de la science. Grâce aux bases de données sur les publications scientifiques, on peut associer les disciplines scientifiques, les affiliations des auteurs et les établissements avec les informations sur les brevets. Par recoupement entre le domaines technologique du brevet contenant la citation et la discipline scientifique dont relève la publication citée, par exemple, on obtient des matrices qui montrent la présence de disciplines scientifiques spécifiques, en relation avec les différents domaines technologiques (Schmoch, 1997; Verbeek et al., 2002).

Le nombre moyen de références scientifiques figurant en première page des brevets d'une société constitue un indicateur simple au niveau de cette société. Un lien scientifique fort indique que la société construit sa technologie grâce aux avancées de la science (" proximité avec la science »). Les sociétés de hautes technologies ont généralement un lien scientifique plus fort que les autres et on a constaté qu'il existait une corrélation entre le lien scientifique et la performance boursière d'une société (voir par exemple Nagaoka, 2007). 


\section{Graphique 6.1. Part de la littérature hors brevets dans les citations des rapports de recherche des demandes de brevets selon le PCT}

1990-2004, par sous-classe CIB $^{1}$

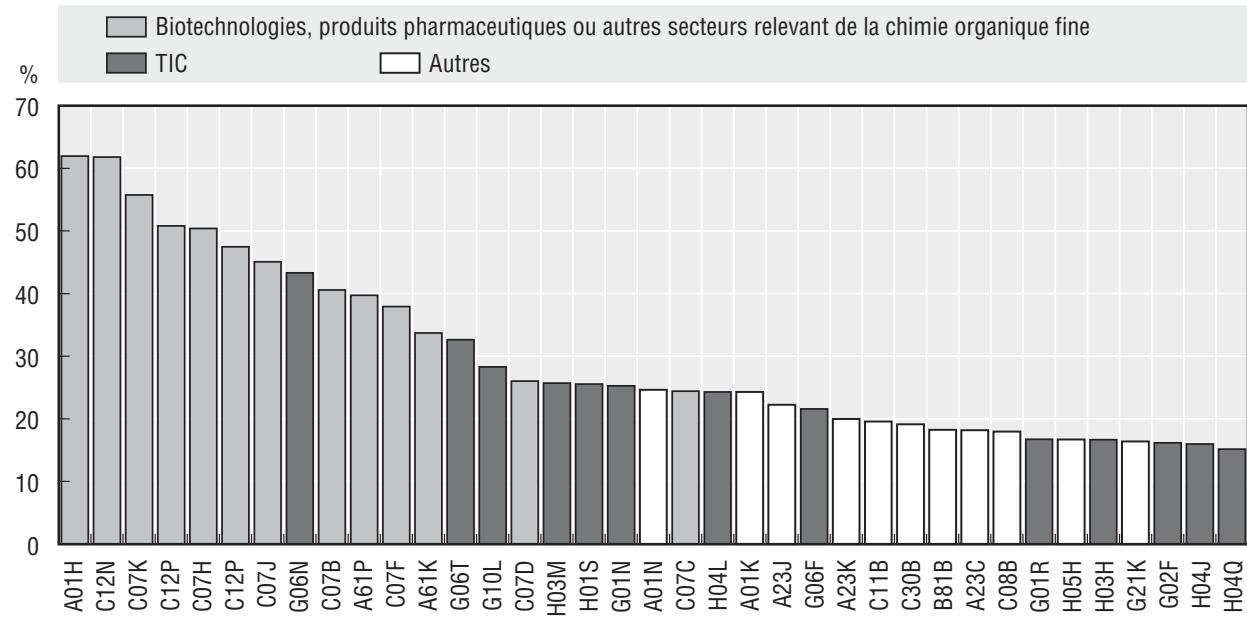

\section{Graphique 6.2. Part du hors brevets dans les citations - tous brevets}

1990-2004, par nationalité de l'inventeur ${ }^{2}$

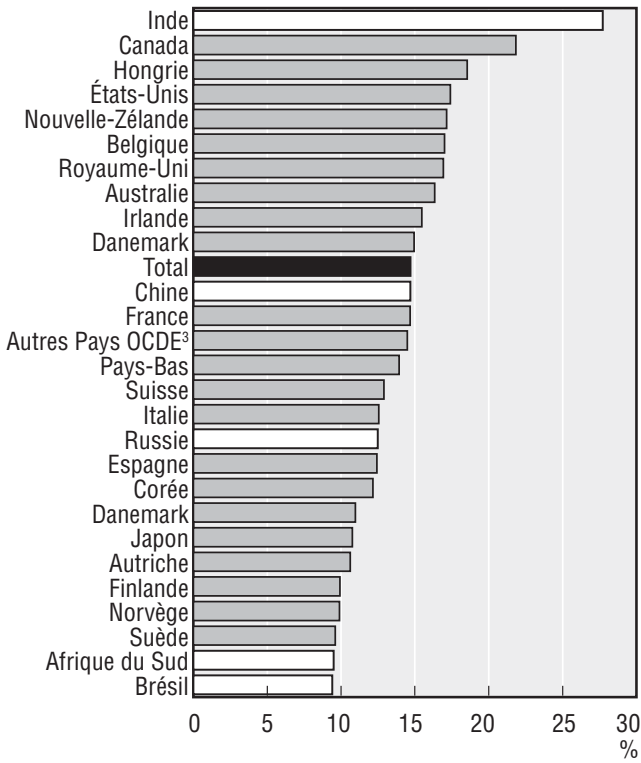

Graphique 6.3. Part du hors brevets dans les citations - TIC

1990-2004, par nationalité de l'inventeur ${ }^{2}$

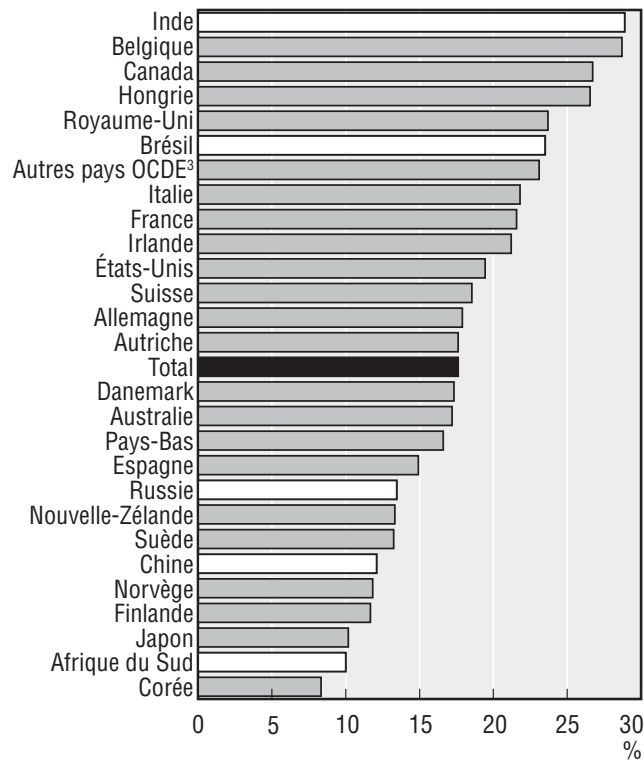

1. Uniquement les sous-classes CIB (sur un total de plus de 600) présentant une part de citations hors brevets plus élevée que la moyenne (14.7\%) et dans lesquelles plus de 150 demandes ont été publiées pendant la période 1990-2004.

2. Comptage fractionnaire lorsqu'il y a plus d'un inventeur pour l'application du brevet.

3. La rubrique Autres OCDE comprend la République tchèque, la Grèce, l'Islande, le Luxembourg, le Mexique, la Pologne, le Portugal, la République slovaque et la Turquie.

Sources: OCDE, base de données de l'OEB sur les citations de brevets. 


\subsection{Autres indicateurs reposant sur les types de citations (rapports de recherche OEB et PCT)}

Dans les rapports de recherche PCT et OEB des codes de pertinence sont affectés aux références décrivant l'état antérieur de la technique d'une invention (Schmoch, 1993). Les publications OEB distinguent cinq types de citations : i) ajoutées par les examinateurs au cours de la recherche (qu'elles aient été fournies ou non par le déposant); ii) fournies par le déposant mais pas reprises dans le rapport de recherche; iii) ajoutées au cours de l'examen; iv) fournies à l'occasion d'une procédure de contestation; v) autres. Tous les documents cités sont identifiés par une lettre en première colonne du rapport de recherche qui représente la catégorie de citation (des combinaisons de codes sont possibles). Voir la définition des catégories de citations dans le tableau 6.4.

\section{Tableau 6.4. Catégories de citations à l'OEB et selon le PCT}

\begin{tabular}{|c|c|}
\hline n & $\begin{array}{l}\text { Document particulièrement pertinent considéré isolément (l'invention revendiquée ne peut être considérée } \\
\text { comme nouvelle ou comme impliquant une activité inventive par rapport au document). }\end{array}$ \\
\hline$Y$ & $\begin{array}{l}\text { L'invention revendiquée ne peut être considérée comme impliquant une activité inventive lorsque le document } \\
\text { est associé à un ou plusieurs autres documents de même nature. }\end{array}$ \\
\hline A & Document définissant l'état général de la technique. \\
\hline 0 & Document renvoyant à une divulgation orale, à un usage, à une exposition ou à tous autres moyens. \\
\hline $\mathbf{P}$ & Document intermédiaire (publié entre la date de dépôt et la date de priorité). \\
\hline $\mathbf{T}$ & $\begin{array}{l}\text { Document ultérieur relatif à la théorie ou au principe constituant la base de l'invention mais ne s'opposant pas } \\
\text { à la demande (cité pour une meilleure compréhension de l'invention). }\end{array}$ \\
\hline $\mathbf{E}$ & $\begin{array}{l}\text { Document susceptible d'entrer en conflit avec la demande, publié à la date du dépôt de l'invention ou après } \\
\text { cette date. }\end{array}$ \\
\hline D & Document déjà cité dans la demande (fourni par le déposant). \\
\hline $\mathbf{L}$ & $\begin{array}{l}\text { Document cité pour une raison spéciale (par exemple document pouvant jeter un doute sur une ou plusieurs } \\
\text { revendications). }\end{array}$ \\
\hline
\end{tabular}

Source: Directives concernant l'examen à l'Office européen des brevets, 2003 (176ff.).

Ce classement peut être utile pour construire des indicateurs plus fins sur les citations, comme les indicateurs sur les brevets susceptibles de bloquer d'autres inventions (basé sur les catégories X, Y et E). Les catégories X et Y, qui désignent la citation d'un document pertinent de l'état antérieur de la technique, sont très importantes pour évaluer la brevetabilité d'une nouvelle invention et peuvent compromettre la délivrance d'un brevet. À cet égard, les références $\mathrm{X}$ sont les plus importantes. Si une demande reçoit un classement $\mathrm{X}$, cela indique que l'invention revendiquée ne remplit pas tout ou partie des impératifs de nouveauté ou d'inventivité, et qu'une revendication au moins doit être modifiée pour éviter d'empiéter sur les limites légales d'autres inventions existantes. Par conséquent, lorsque l'on observe des brevets qui ont été délivrés et qui contiennent des citations en amont de ce type, les revendications qui apparaissent dans la version finale ont dû, le plus souvent, 
être modifiées pendant la procédure de délivrance du brevet. Dans le rapport de recherche, l'examinateur ou le spécialiste de la recherche précise quelles revendications de l'invention posent problème au regard de l'état antérieur de la technique.

Les documents cités par le déposant (type D) doivent être examinés dans le rapport de recherche soit s'ils ont un caractère décisif pour l'état de la technique, soit s'ils sont nécessaires pour comprendre l'invention. Les citations soumises par le déposant qui ne répondraient pas à l'une de ces conditions peuvent être ignorées par l'examinateur. Les références de type $\mathrm{A}$ fournissent simplement des informations techniques de référence (état antérieur de la technique). Le fait qu'un brevet soit fréquemment cité comme invalidant totalement ou partiellement d'autres applications brevetées peut aussi révéler des pratiques tactiques en matière de brevet, les titulaires ayant conçu leur application de manière à bloquer ou à freiner la brevetabilité d'inventions postérieures par des concurrents.

\section{Notes}

1. Dans une recherche menée sur les titulaires de brevets et les inventeurs, pour environ la moitié de l'ensemble des citations de brevets (dans une cohorte de 1993 titulaires auprès de l'USPTO) il n'y a eu aucune communication, et on n'a observé aucune relation technologique perceptible entre les inventions (ex. Jaffe et al., 2000).

2. Les demandes auprès de l'USPTO publiées (intitulées "pre-grant publications") contiennent les citations fournies par le déposant, mais pas celles de l'examinateur. Ces dernières ne sont publiées que si et quand le brevet est accordé.

3. Pour plus de détail, voir les Directives relatives à l'examen pratiqué à l'Office européen des brevets, qui sont actualisées régulièrement. Après une partie générale, ces directives sont divisées en cinq parties parmi lesquelles les directives relatives à l'examen des demandes quant à leur forme, les directives quant à la recherche et les directives relatives à l'examen des demandes quant au fond.

4. Si la recherche aboutit à plusieurs documents de pertinence équivalente, le rapport de recherche ne doit en principe pas en contenir plus d'un. Le choix de celui qui doit être cité est fait sur la base de l'expertise de l'examinateur. S'il y a deux documents de pertinence équivalente, dont l'un a été publié avant la date de priorité et l'autre entre la date de priorité et la date de dépôt, l'examinateur doit choisir le premier.

5. Comme le montrent Callaert et al. (2006) dans une étude comparative des brevets USPTO et OEB, ces différences apparaissent non seulement au niveau du nombre d'occurrence mais aussi du type de références citées. Les brevets USPTO comprennent en moyenne environ trois fois plus de références que les brevets $O E B$. S'agissant de la littérature hors brevets, $34 \%$ des brevets USPTO y font référence, contre $38 \%$ à l'OEB. Ils constatent également que les références aux revues scientifiques sont plus nombreuses dans les brevets OEB (64\% des références hors brevets portent sur des revues scientifiques, contre $54 \%$ dans les brevets USPTO). 
6. Pour les brevets qui entrent en phase régionale à l'OEB par la suite, l'OEB joue le rôle d'ISA. D'un point de vue formel, le rapport de recherche international joue un rôle différent de celui émis par l'OEB pour les demandes déposées directement auprès de l'OEB. D'un point de vue pratique, il y a peu de différences. Les rapports de recherche internationale des documents WO sont produits par l'une des 12 ISA, à savoir les bureaux des brevets de l'Australie, de l'Autriche, de la Chine, de la Corée, de l'Espagne, des États-Unis, de la Finlande, du Japon, de la Russie, de la Suède ou l'Office européen des brevets.

7. Pour en savoir plus, voir les Directives concernant la recherche internationale et l'examen préliminaire international de l'OMPI, www.wipo.int/pct/fr/texts/pdf/ispe.pdf.

8. Dans le contexte des citations de brevets de l'OEB, si une invention est protégée dans plus d'un pays et donc, si une même famille de brevets comprend plusieurs documents, l'examinateur doit de préférence citer le document rédigé dans la même langue que la demande. Le choix est également affecté par les langues connues de l'examinateur. Lorsque les documents de brevets ne sont pas cités dans les références comme européens, mais sous forme de documents émis par d'autres bureaux des brevets (l'OMPI, l'USPTO, le DPMA entre autres), le comptage des citations éclaire l'analyste quant à la source des références, mais pas quant à l'importance d'inventions particulières.

9. La propension à citer varie aussi dans le temps et d'un domaine technologique à l'autre. C'est dans le domaine de l'informatique et des communications que les citations sont les plus rapprochées des brevets cités, suivies par l'électricité et l'électronique, puis par les médicaments et les technologies médicales (Hall et al., 2001).

10. En vertu du American Inventors Protection Act (AIPA), en vigueur depuis le 29 novembre 1999, tous les brevets pour lesquels une forme de protection à l'extérieur des États-Unis est demandée sont publiés par l'USPTO, encore une fois 18 mois après la date de priorité aux États-Unis. Cela ne change pas la date de la première publication, mais la publication est maintenant accessible auprès de l'USPTO et apparaîtra dans les données même si les équivalents européens du brevet américain ne sont pas détectés.

11. Si un brevet est cité par des brevets ultérieurs qui appartiennent à de nombreux domaines différents, la mesure sera élevée (proche de un), alors que si la plupart des citations sont concentrées dans un petit nombre de domaines, elle sera faible (proche de zéro).

12. La méthode de calcul de la magnitude du biais (et de la correction du biais) sont expliqués dans Hall et al. (2001).

13. Cela coïncide avec les autres aspects des liens entre science et industrie dans ces domaines, comme les entreprises nées de la recherche universitaires, les collaborations entreprises-universités en R-D et la tendance des sociétés de biotechnologies à s'implanter à proximité des universités.

\section{Références}

Callaert J., B. Van Looy, A. Verbeek, K. Debackere et B. Thijs (2006), « Traces of Prior Art. An Analysis of Non-Patent References Found within Patent Documents ", Scientometrics, vol. 69, $n^{\circ} 1$, avril, pp. 3-20.

Hall B.H., A. Jaffe et M. Trajtenberg (2001), « Market Value and Patent Citations: A First Look », Economics Department Working Paper E00-277, University of California. 
Hall, B.H., A. Jaffe et M. Trajtenberg (2005), « Market Value and Patent Citations », Rand Journal of Economics 36, printemps.

Harhoff, D., K. Hoisl et C. Webb (2006), « European Patent Citations - How to Count and How to Interpret Them? ", mimeo, août.

Harhoff, D., F.M. Scherer et K. Vopel (2002), Citations, Family Size, Opposition and the Value of Patent Rights, Research Policy, Elsevier, 32(8), pp. 1343-1363.

Jaffe, A.B., M. Trajtenberg et R. Henderson (1993), "Geographic Localization of Knowledge Spillovers as Evidenced by Patent Citations ", Quarterly Journal of Economics 108, pp. 577-598.

Jaffe, A.B., M. Trajtenberg et M.S. Fogarty (2000), « Knowledge Spillovers and Patent Citations: Evidence from a Survey of Inventors ", American Economic Review, American Economic Association, vol. 90(2), pp. 215-218.

Lanjouw, J.O. (1998), « Patent Protection in the Shadow of Infringement: Simulation Estimations of Patent Value ", The Review of Economic Studies, vol. 65, pp. 671-710.

Lanjouw, J.O., A. Pakes et J. Putnam (1998), « How to Count Patents and Value Intellectual Property: Uses of Patent Renewal and Application Data ", The Journal of Industrial Economics, vol. XLVI, $n^{\circ} 4$, December, pp. 405-433.

Lanjouw, J.O. et M. Schankerman (1997), «Stylised Facts of Patent Litigation: Value, Scope and Ownership ", NBER working paper series, NBER, Cambridge, MA.

Malerba, F. et L. Orsenigo (1995), "Schumpeterian Patterns of Innovation », Cambridge Journal of Economics, Oxford University Press, vol. 19 (1), février, pp. 47-65.

Meyer, M. (2000), « Does Science Push Technology? Patents Citing Scientific Literature », Research Policy, vol. 29, $\mathrm{n}^{\circ}$ 3, mars, pp. 409-434.

Michel, J. et B. Bettels (2001), « Patent Citation Analysis - A closer look at the basic input data from patent research reports ", Scientometrics 51, pp. 181-201.

Nagaoka, S. (2007), "Assessing the R\&D Management of a Firm in Terms of Speed and Science Linkage: Evidence from the US Patents », Journal of Economics and Management Strategy, vol. 16, $\mathrm{n}^{\circ}$ 1, printemps, pp. 129-156.

Narin, F., K. Hamilton et D. Olivastro (1997), " The Increasing Linkage between US Technology and Public Science », Research Policy, 26, pp. 317-330.

Podolny, J.M., T.E. Stuart et M.T. Hannan (1996), « Networks, Knowledge and Niches: Competition in the Worldwide Semiconductor Industry, 1984-1991 », American Journal of Sociology 102 (3), novembre, pp. 659-689.

Sampat, B.N. et A. Ziedonis (2004), "Patent Citations and the Economic Value of Patents: A Preliminary Assessment », avec B.N. Sampat in H. Moed, W. Glänzel and U. Schmoch (éd.), Handbook of Quantitative Science and Technology Research: The Use of Publication and Patent Statistics in Studies on R\&D Systems, Kluwer Academic Publishers, Dordrecht/Boston/Londres, pp. 277-298.

Schmoch, U. (1997), "Indicators and the Relations between Science and Technology ", Scientometrics, vol. 38 (1), pp. 103-116.

Schmoch, U. (1993), « Tracing the Knowledge Transfer from Science to Technology as Reflected in Patent Indicators ", Scientometrics, vol. 26, (1), pp. 193-211.

Tijssen, R. J. W. (2002), "Science Dependence of Technologies: Evidence from Inventions and their Inventors ", Research Policy 31, pp. 509-526. 
Trajtenberg, M., R. Henderson et A. Jaffe (1997), « University versus Corporate Patents: A Window on the Basicness of Invention ", Economics of Innovation and New Technology, vol. 5 (1), pp. 19-50.

Trajtenberg, M. (1990), «A Penny for Your Quotes: Patent Citations and the Value of Innovation ", RAND Journal of Economics, 21 (1), pp. 172-187.

Verbeek, A., K. Debackere et M. Luwel (2002), « Science Cited in Patents: A Geographic " Flow » Analysis of Bibliographic Citation Patterns in Patents », Scientometrics 58 (2), pp. 241-263 (2003).

Webb, C., H. Dernis, D. Harhoff et K. Hois (2005), « Analysing European and International Patent Citations: A Set of EPO Patent Database Building Blocks ", OECD Science, Technology and Industry Working Paper 2005/9, Direction de la science, de la technologie et de l'industrie, OCDE, Paris, www.oecd.org/sti/working-papers. 
Chapitre 7

Indicateurs de l'internationalisation de la science et de la technologie 


\subsection{Introduction}

Les activités d'invention se déroulent de plus en plus à l'échelle internationale (OCDE, 2007). Ainsi, les inventions réalisées par des chercheurs résidant dans un pays peuvent être financées et détenues par des entreprises étrangères, des sociétés de pays distincts peuvent mettre en commun leurs ressources pour financer la recherche, et des chercheurs de divers pays collaborer pour développer des inventions. Des alliances sont créées entre différents sites pour dégager des synergies et des complémentarités dans la recherche et acquérir de nouvelles compétences technologiques. L'apparition des chaînes de valeur mondiales, les différences de coûts de la recherchedéveloppement (R-D), la flexibilité accrue de la gestion de projets de R-D transnationaux (grâce aux technologies de l'information et de communication TIC) et des modifications notables de la politique menée (comme le renforcement des droits de propriété intellectuelle ou le régime fiscal de la R-D) ont favorisé ce phénomène. Compte tenu de l'importance de ces changements et de leurs conséquences pour la capacité technologique des pays, il y a lieu de quantifier l'intensité et la répartition géographique de ces activités.

On dispose de différents indicateurs pour mesurer l'internationalisation de la science et de la technologie (S-T). Ils sont fondés notamment les statistiques de R-D et du commerce international, comme la part de la R-D financée par des sources étrangères, les exportations et les importations de produits de haute technologie, et les sommes versées et perçues dans les services technologiques (OCDE, 2005). Concernant l'internationalisation des activités de R-D, l'analyse repose sur des données tirées d'enquêtes relatives aux activités des entreprises multinationales et d'études de cas. Les enquêtes menées auprès des entreprises fournissent des indications précieuses sur les activités des filiales (base de données AFA de l'OCDE), mais elles demeurent limitées à quelques pays.

L'internationalisation des activités technologiques peut aussi être évaluée à l'aide des résultats concrets des activités d'invention, mesurés par les brevets. Les documents de brevets font apparaître le nom de l'inventeur (ou des inventeurs) et du demandeur (ou des demandeurs) - le propriétaire du brevet à la date de la demande de dépôt - ainsi que leur adresse, et donc leur pays de résidence. L'exploitation de ces informations, séparément ou conjointement, peut être révélatrice de l'organisation géographique des inventions. C'est ce que montrent les indicateurs présentés dans ce chapitre. On peut également recourir à 
d'autres informations, comme les citations : les brevets citant d'autres brevets correspondant à des inventions développées dans un autre pays traduisent les flux internationaux de connaissances. Les indicateurs fondés sur les citations ont été présentés au chapitre 6 et ne seront pas abordés ici.

\subsection{Indicateurs}

\subsubsection{Propriété transnationale des inventions}

Lorsque le pays de résidence du demandeur diffère de celui de l'inventeur, on parle de propriété transnationale. À partir des informations figurant directement ou indirectement dans les documents de brevets, il est possible d'établir deux indicateurs de la propriété transnationale à l'échelle des pays ou des régions (Guellec et van Pottelsberghe, 2001) :

- Propriété étrangère des inventions nationales: il s'agit du nombre de brevets délivrés à des demandeurs résidant à l'étranger (pays de référence $i$, pays étranger $j=1, \ldots . N, j=i)$ et comptant au moins un inventeur résident $\left(P_{i, j}\right)$, divisé par le nombre total de brevets correspondant à des inventions nationales $\left(P_{i}\right)$. Pour le pays $i$, la part de la propriété étrangère dans l'invention nationale totale est donc de : $\frac{j_{j=1}^{N} \sum P_{i, j}}{P_{i}}$.

- Propriété nationale des inventions étrangères: il s'agit du nombre de brevets attribués dans un pays, mais dont les inventions ont été réalisées à l'étranger et qui comptent au moins un inventeur étranger $\left(P_{i, j}\right)$, divisé par le nombre total de brevets détenus dans le pays $\left(P_{i}\right)$. Pour le pays $i$, la part de la propriété nationale dans le total des brevets détenus est donc de : $\frac{{ }_{j=1}^{N} \sum P_{i, j}}{P_{i}}$.

Dans la plupart des cas, les brevets comptant des inventeurs étrangers correspondent à des inventions réalisées dans les laboratoires de recherche des entreprises multinationales et pour lesquelles une demande a été déposée au siège de l'entreprise (bien que des filiales nationales puissent aussi être propriétaires ou copropriétaires des brevets). Par conséquent, le premier indicateur est révélateur du contrôle exercé par les entreprises étrangères sur les inventions nationales. Par analogie, le second met en évidence le contrôle exercé par les entreprises nationales sur les inventions réalisées par des résidents d'autres pays ${ }^{1}$. Ces indicateurs montrent donc le rôle joué par les filiales étrangères des entreprises multinationales dans les activités d'innovation. Ils viennent compléter les données sur la R-D de ces filiales. Ce contrôle exercé par des intérêts étrangers indique que plusieurs pays bénéficient des avantages économiques découlant des inventions: le pays où l'invention a été réalisée, 
celui où elle est détenue, mais aussi en partie d'autres pays, puisque les entreprises multinationales peuvent déployer un partie de leur technologie à l'échelle mondiale (à travers la fabrication ou la commercialisation).

\subsubsection{Recherche et coopération internationale}

On peut aussi évaluer l'internationalisation de la technologie à l'aide de la coopération internationale menée dans la recherche, mesurée par les brevets mettant en présence des inventeurs résidant dans des pays distincts. Il s'agit du nombre de brevets délivrés pour des inventions nationales (pays de référence $i$, pays étranger $j=1, \ldots . N, j=i$ ) et dont un inventeur au moins réside dans un pays étranger $\left(P_{i, j}\right)$, rapporté au nombre total de brevets correspondant à des inventions nationales $\left(P_{i}\right)$. Pour le pays $i$, la part des co-inventions internationales dans le total des inventions nationales est donc de : $\frac{{ }_{j=1}^{N} \sum P_{i, j}}{P_{i}}$.

La spécialisation et le capital-savoir différant selon les pays, il est possible de trouver des connaissances externes complémentaires à l'étranger. Les chercheurs peuvent collaborer à l'échelle mondiale au sein d'une multinationale (comptant des centres de recherche dans plusieurs pays) ou d'un projet de recherche en coopération réunissant plusieurs entreprises ou établissements (collaboration entre des inventeurs appartenant à des universités ou à des établissements publics de recherche différents). En ce sens, les indicateurs de coinvention rendent également compte des flux internationaux de connaissance.

Les indicateurs de propriété transnationale et de co-invention sont étroitement liés. La co-invention internationale, par définition, implique une propriété transnationale. En fait, cette propriété transnationale se décompose en inventions impliquant ou pas une co-invention (le pays du demandeur étant aussi celui d'un inventeur). Naturellement, quand il y a propriété étrangère dans le pays d'un inventeur, cela implique que dans un autre pays, une invention nationale est détenue par des entreprises nationales. En toute logique, les totaux mondiaux sont nettement plus bas que les chiffres communiqués par certains pays, puisque les comptages sont consolidés.

Le graphique 7.1 retrace l'évolution de la propriété transnationale et de la co-invention à l'échelle mondiale sur la période 1990-2002 d'après les demandes de brevet déposées auprès de l'Office européen des brevets (OEB). La propriété transnationale correspond au pourcentage de brevets dont un inventeur au moins réside dans un pays distinct de celui du propriétaire (par rapport au total des inventions mondiales), tandis que la co-invention désigne la fraction du total des inventions mondiales correspondant aux brevets dont deux inventeurs au moins résident dans des pays différents. Sur cette période, la co-invention internationale a été multipliée par plus de deux, grimpant 


\section{Graphique 7.1. La mondialisation de la S-T}

Demandes de brevet déposées auprès de l'OEB-PCT de 1990 à 2002

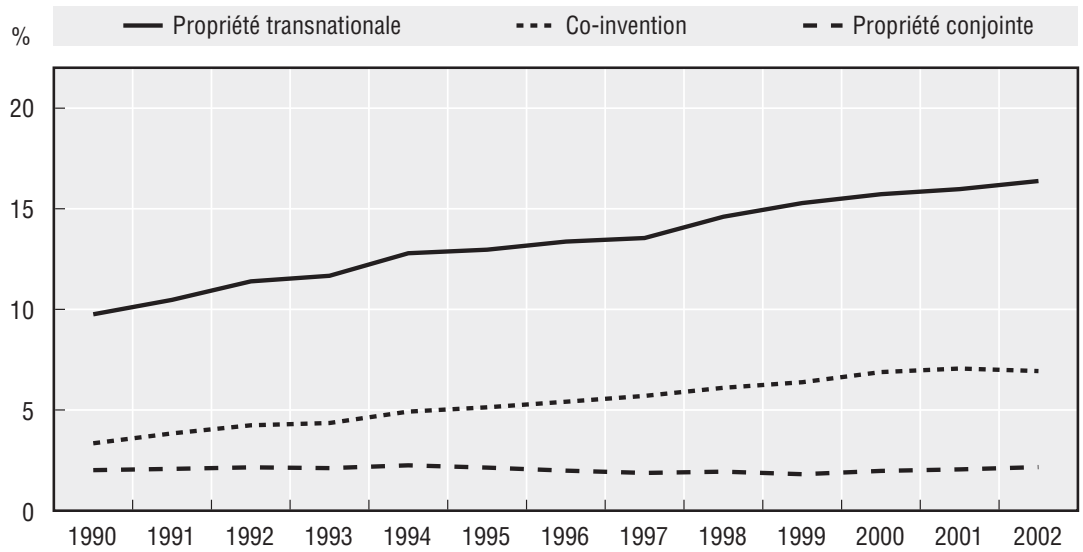

Note: Le comptage des brevets est basé sur le pays de résidence de l'inventeur, la date de priorité et le comptage fractionnaire. Demandes de brevet déposées conformément au Traité de coopération en matière de brevets (PCT) et désignant l'Office européen des brevets. Propriété transnationale : fraction du total des inventions mondiales correspondant aux brevets dont un demandeur réside dans un pays distinct de celui de l'inventeur. Co-invention: fraction du total des inventions mondiales correspondant aux brevets dont deux inventeurs au moins résident dans des pays différents. Propriété conjointe : part des brevets pour lesquels deux codemandeurs au moins résident dans des pays distincts (dans le total des inventions mondiales).

Source : OCDE, base de données sur les brevets, mai 2006.

ainsi de quelque $3 \%$ en 1990 à plus de $7 \%$ en 2002. La part de la propriété transnationale a progressé régulièrement à l'échelle mondiale, soit de $50 \%$ entre le début des années 90 et le début des années 2000. En 2002, sur dix brevets déposés auprès de l'OEB, plus de 1.5 relevait de la propriété transnationale. Les graphiques 7.2 et 7.3 présentent pour plusieurs pays les indicateurs relatifs à la propriété étrangère des inventions nationales et à la propriété nationale des inventions étrangères.

\subsubsection{Avantages et réserves liés à l'utilisation des brevets aux fins de la mesure de l'internationalisation de la S-T}

Utiliser des indicateurs de brevets pour suivre l'internationalisation de la technologie présente de nombreux avantages. Les brevets fournissent entre autres choses une description relativement complète de l'invention, du domaine technologique concerné, de l'inventeur (nom, situation géographique, etc.), du demandeur, des références à ou des citations de brevets antérieurs et des articles scientifiques en relation avec l'invention. L'internationalisation de la technologie peut être évaluée en fonction du domaine technologique, du type d'entreprise (lorsqu'on dispose de données sur les entreprises, sur leur taille notamment), des liens existant entre les universités et le secteur, etc. 


\section{Graphique 7.2. Propriété étrangère des inventions nationales}

Demandes de brevet déposées auprès de l'OEB-PCT de 1991 à 2003

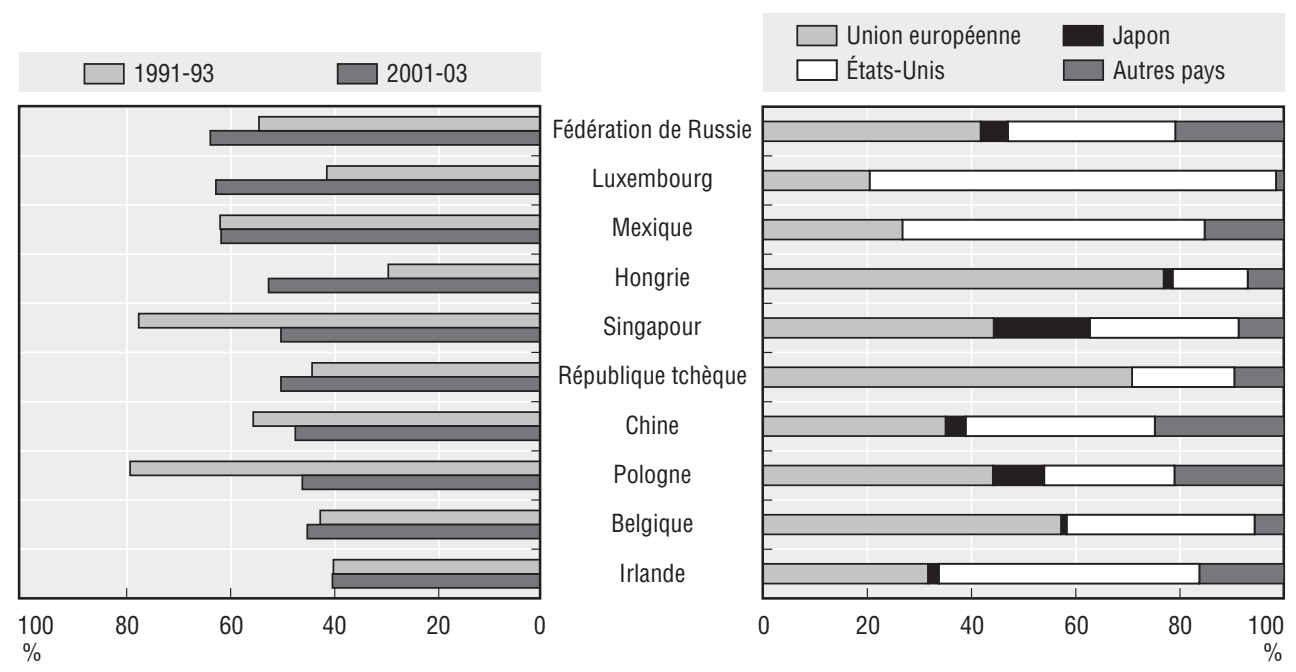

Note: Les comptages de brevets sont basés sur la date de priorité et le pays de résidence de l'inventeur, à l'aide de comptages simples; fraction du total des brevets d'inventions nationales correspondant à des demandes de brevet déposées auprès de l'Office européen des brevets et détenues par des résidents étrangers.

Source : OCDE, base de données sur les brevets.

\section{Graphique 7.3. Propriété nationale des inventions étrangères}

Demandes de brevet déposées auprès de l'OEB-PCT de 1991 à 2003
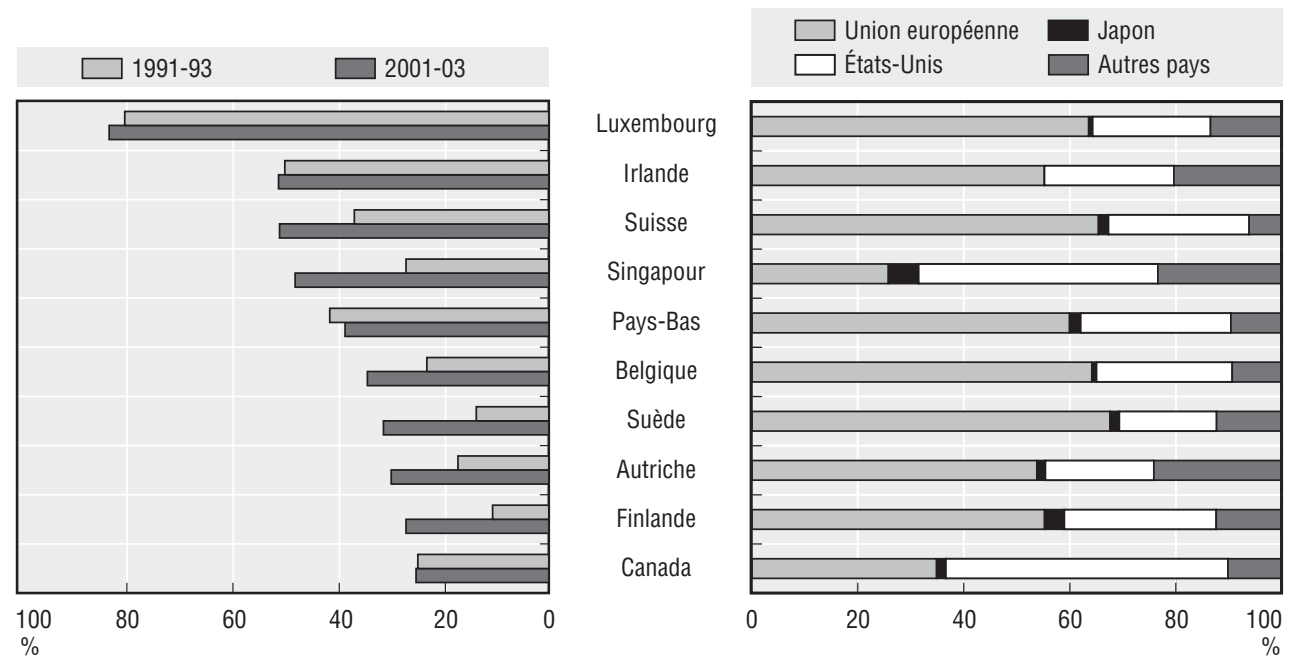

Note : Le comptage des brevets est basé sur la date de priorité et le pays de résidence du demandeur, à l'aide de comptages simples. Fraction du total des brevets détenus par des résidents correspondant à des demandes de brevet déposées auprès de l'Office européen des brevets (OEB) pour des inventions étrangères.

Source: OCDE, base de données sur les brevets. 
Les principales difficultés liées à la mesure de l'internationalisation de la technologie à l'aide des renseignements relatifs aux brevets tiennent à la complexité de la structure capitalistique et de la stratégie des entreprises, ainsi qu'à l'absence d'informations sur ces points, de sorte qu'il est parfois malaisé de déterminer le pays d'origine de la société propriétaire mentionnée dans le dossier du brevet. Nombre de ces difficultés sont néanmoins dues au simple fait de déterminer le pays de la société, problème qui existe pour tous les indicateurs de l'internationalisation (OCDE, 2005). Plusieurs cas de figure peuvent prêter à confusion :

- Le propriétaire mentionné dans le dossier du brevet peut être la filiale d'un groupe multinational chargée de gérer sa propriété intellectuelle internationale, et non l'entreprise multinationale elle-même. Cette filiale pouvant être située dans un autre pays que celui du siège social du groupe (pour des raisons stratégiques ou fiscales), la représentation des liens transnationaux en est faussée.

- Une invention brevetée peut être contrôlée ex post par une entité étrangère, par exemple lorsque son propriétaire d'origine est racheté par cette entité étrangère ou fusionne avec elle, ou quand les droits de brevet sont transférés à cette entité. La situation inverse (une entreprise détenue par des intérêts étrangers devient une entreprise nationale) peut également se produire. Il se peut que le nouveau propriétaire prenne directement le contrôle du brevet. Les bases de données classiques sur les brevets n'enregistrent pas ces changements de propriétaire lorsqu'ils surviennent après la délivrance du titre, donnant ainsi une image imprécise du contrôle effectif des inventions.

- Le brevet peut être détenu (ou déposé) directement par la filiale nationale d'un groupe multinational, qui n'est donc pas mentionné en tant que tel dans le dossier du brevet (voir le chapitre 5). En pareil cas, la propriété étrangère est sous-estimée (voir, par exemple, le cas de la Belgique dans Cincera et al., 2006) et la propriété nationale des inventions étrangères l'est également dans les mêmes proportions en ce qui concerne le pays du propriétaire.

Il convient de faire preuve de prudence pour interpréter l'activité de coinvention internationale à l'aide des brevets. Les inventeurs résidant dans des pays différents travaillent souvent pour la même entreprise multinationale et ses pratiques de gestion peuvent influer sur le choix de l'inventeur qui sera mentionné (ou premier inventeur, voir Bergek et Bruzelius, 2005). Inversement, des inventeurs résidant à l'étranger peuvent également intervenir lorsque la recherche est externalisée.

Il n'est pas nécessaire de communiquer des informations sur les entreprises (pays d'origine et propriétaires internationaux des entreprises) lors du dépôt de la demande. Lorsqu'ils sont calculés à partir des seuls éléments contenus dans les 


\section{Encadré 7.1. Dispersion régionale des brevets}

Les indicateurs présentés dans ce chapitre peuvent être établis pour des régions ou des pays : ils mesurent alors la propriété et la coopération transrégionales. Bien que les formules soient similaires, l'interprétation économique peut différer quelque peu puisque les différences et les barrières existant entre les régions sont habituellement bien moins importantes qu'entre les pays (langue, réglementation, système fiscal, distance, par exemple). L'adaptation de la technologie existante aux préférences locales, qui est l'une des grandes raisons motivant l'implantation de la R-D plus près de la demande finale, s'applique davantage à l'investissement transnational qu'à l'investissement transrégional.

L'OCDE utilise la classification des niveaux territoriaux, qui comporte différents niveaux d'agrégation (le niveau 2 comprend environ 300 macrorégions, et le niveau 3 plus de 2300 régions, comme les "economic areas » américaines définies par le Bureau of Economic Analysis, les "préfectures " japonaises, les départements français, etc.). Dans les pays de l'UE, les régions sont définies selon la nomenclature NUTS (Nomenclature des unités territoriales statistiques), une classification officielle établie par la Commission européenne (qui est équivalente à la classification de l'OCDE pour l'Europe).

documents de brevet, les indicateurs présentés ici peuvent sous-estimer l'importance de l'internationalisation. Il est recommandé de compléter les renseignements relatifs au propriétaire fournis par les bases de données sur les brevets par d'autres informations concernant la propriété de l'entreprise, afin d'obtenir des indicateurs plus précis de l'internationalisation de la technologie.

\subsection{Propriété et stratégies de recherche}

La mondialisation des activités technologiques, ainsi qu'en témoignent les brevets, est à la fois le fait de stratégies de recherche et de propriété. Les données relatives aux brevets donnent une compréhension plus fine de ces questions lorsqu'on s'intéresse à l'articulation des types de propriété (ou de propriété conjointe) et d'invention (inventions réalisées uniquement par des non-résidents ou co-invention). On peut dégager de ces statistiques cinq schémas d'internationalisation :

1. Propriété exclusivement nationale des inventions étrangères (propriétaire résidant dans le pays A et inventeur dans le pays B). Ce type de stratégie correspond à des brevets pour lesquels la recherche a été menée intégralement dans le laboratoire étranger (filiale d'une entreprise multinationale).

2. Propriété nationale impliquant une propriété conjointe avec un seul inventeur (propriétaires résidant dans les pays A et B et inventeur dans le pays B 
exclusivement). Ce schéma peut correspondre à des brevets détenus conjointement par la multinationale et une filiale située à l'étranger, ou par un établissement de recherche créé sous forme de co-entreprise par des sociétés de deux pays différents.

3. Propriété nationale avec co-invention (inventeurs résidant dans les pays A et $\mathrm{B}$, mais propriétaire résidant dans le pays $A$ uniquement). Ce schéma concerne des brevets déposés par des entreprises multinationales poursuivant une double stratégie d'internationalisation : exploitation de leur capital-savoir et accès à des connaissances étrangères.

4. Propriété conjointe associée à la co-invention (inventeurs et propriétaires résidant dans les pays $A$ et $B$ ). Cette stratégie concilie les deux schémas précédents. Elle peut résulter de la participation conjointe du siège et d'une filiale étrangère d'une entreprise multinationale, ou d'un projet de recherche en coopération entre des entreprises de deux pays distincts.

5. Paternité de l'invention ou propriété transnationale, et inventeur et propriétaire résidant dans des pays différents (propriétaires dans les pays A et B et inventeur dans le pays $C$ ). Ce schéma complexe et peu courant nécessite une analyse au cas par cas. Il peut être le fait d'un réseau international d'entreprises ayant confié la gestion de leurs brevets à une troisième (groupements technologiques, par exemple).

\section{Graphique 7.4. Composition de la propriété transnationale dans les demandes de brevet déposées}

Demandes de brevet déposées auprès de l'OEB-PCT

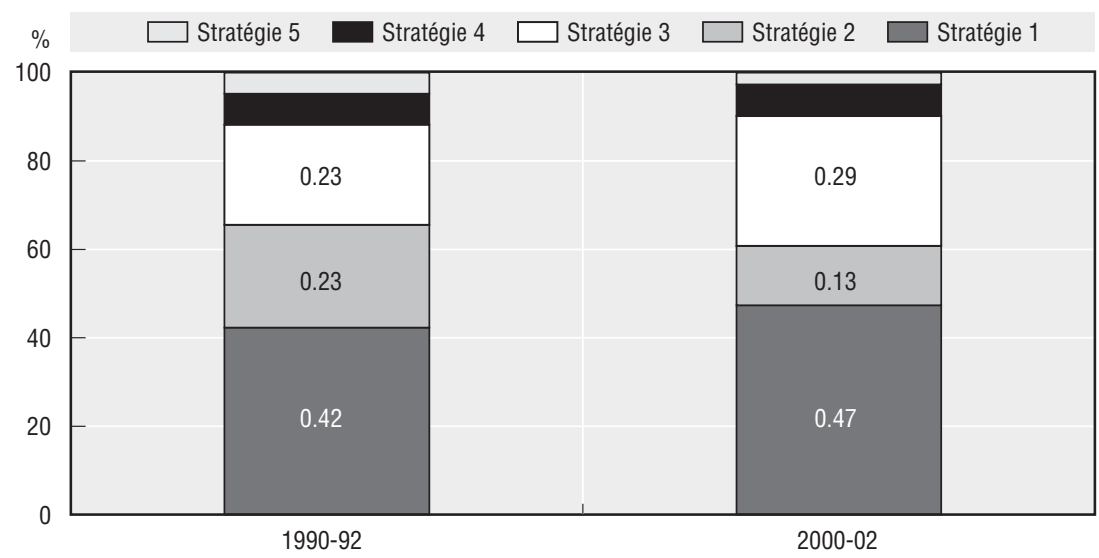

Note: Comptages fractionnaires des demandes de brevet déposées conformément au Traité de coopération en matière de brevets (Patent Cooperation Treaty, PCT) et désignant l'Office européen des brevets, par stratégie et année de priorité.

Source : OCDE, base de données sur les brevets. 
Le graphique 7.4 décompose les brevets soumis à la propriété transnationale. Il semble que la stratégie qui domine les inventions transnationales repose sur l'unicité du pays du propriétaire et de celui de l'inventeur : $47 \%$ des brevets relevant de la propriété transnationale entrent dans cette catégorie, suivis des brevets détenus dans un seul pays avec co-invention (le pays du propriétaire étant aussi celui de l'un des inventeurs). La part de ces derniers est passée de 23 \% en 1990-92 à $29 \%$ en 2000-02, ce qui atteste du déploiement croissant des stratégies mixtes (création de partenariats stratégiques en vue de dégager des synergies technologiques à des fins d'innovation, notamment).

Les trois configurations restantes (propriété et co-invention, propriété conjointe et co-invention, et propriété conjointe avec paternité de l'invention dans un troisième pays) sont moins importantes et de fait, elles ont reculé en proportion : les brevets impliquant une propriété conjointe dans deux pays distincts (multinationales et filiales, ou deux sociétés non apparentées situées dans des pays différents avec paternité de l'invention dans un seul pays ou coinvention) représentent moins de $11 \%$ des brevets transnationaux. Enfin, les brevets détenus conjointement dans des pays distincts, mais sans que l'inventeur réside dans l'un de ces pays, ressortent à moins de $2 \%$.

\section{Note}

1. Une partie de ces brevets qui relèvent de la propriété transnationale peut aussi être détenue conjointement par deux entreprises situées dans des pays distincts; mais là encore, il s'agit davantage de propriété conjointe par le siège social et une ou plusieurs filiales étrangères. Toutefois, cela représente une fraction infime du total des brevets entrant dans le cadre de la propriété transnationale.

\section{Références}

Bergek, A. et M. Bruzelius (2005), « Patents with Inventors from Different Countries: Exploring Some Methodological Issues through a Case Study ", présenté lors de la conférence « DRUID », Copenhague, 27-29 juin.

Guellec, D. et B. van Pottelsberghe (2001), « The Internationalisation of Technology Analysed with Patent Data ", Research Policy, 30 (8), 1256-1266.

OCDE (2005), « Compendium of Patent Statistics 2005 », OCDE, Paris.

OCDE (2007), « Compendium of Patent Statistics 2007 », OCDE, Paris. 
Chapitre 8

\section{Indicateurs de la valeur des brevets}




\subsection{Introduction}

La notion de "valeur des brevets " revêt plusieurs acceptions. Elle peut renvoyer à la valeur économique "privée " apportée au titulaire, telle que définie par les flux actualisés de revenus générés par le brevet pendant sa durée de vie. Elle peut aussi désigner la valeur «sociale » du brevet, c'est-àdire sa contribution au stock technologique de la société. Ces deux concepts sont étroitement liés, puisque les revenus générés doivent être proportionnels à la contribution technologique, mais ils ne sont pas identiques, car une partie de la valeur sociale échappe au titulaire du brevet (en raison de la présence d'externalités) : ainsi, les connaissances publiées peuvent être utilisées par d'autres inventeurs ou par des concurrents, afin d'améliorer l'invention initiale.

De plus, il convient d'établir une distinction entre la valeur du brevet luimême et la valeur de l'invention sous-jacente. La première recouvre uniquement la valeur ajoutée due à la protection de l'invention par un brevet - c'est la différence entre la valeur de l'invention brevetée et celle qu'elle aurait en l'absence de brevet. La deuxième correspond au contenu technologique ou « qualité » de l'invention, c'est-à-dire à sa contribution à l'état de la technique. Une invention dont la contribution est importante va influer sur l'évolution technologique à venir. Ces deux notions diffèrent dans la mesure où le brevet améliore plus l'appropriabilité des avantages de certaines inventions que celle des autres ${ }^{1}$. On sait néanmoins que la capacité des brevets à assurer l'appropriabilité des revenus générés par les inventions varie, par exemple, d'un domaine technique à un autre.

Ces considérations montrent que la valeur d'un brevet est une notion complexe : elle doit néanmoins être prise en compte lorsqu'on réfléchit aux statistiques sur les brevets qui visent à rendre compte des performances technologiques. Toutes les études consacrées à la valeur des inventions brevetées ou de la protection par les brevets montrent que leur distribution statistique est fortement asymétrique; si certains brevets ont une valeur élevée, d'autres n'en ont guère (parce qu'ils ne sont pas exploités, par exemple). En conséquence, les comptages de brevets, qui accordent la même pondération à l'ensemble des brevets, peuvent induire en erreur : un chiffre de 100 brevets peut correspondre à des performances technologiques diverses, selon qu'il est composé de brevets à forte ou à faible valeur ajoutée. Lorsqu'on dispose d'informations sur la valeur des brevets, on peut intégrer ce problème de deux manières dans les indicateurs : la première solution consiste à établir des 
comptages pondérés, en fonction de la valeur; la seconde à comptabiliser uniquement les brevets ayant une valeur suffisante, en laissant les autres de côté.

L'une des difficultés liées à l'estimation de la valeur d'un brevet est l'actualité, c'est-à-dire le fait de disposer d'indicateurs fiables traduisant la valeur économique ou technologique d'une invention suffisamment tôt pour pouvoir s'en servir afin d'évaluer la situation récente d'une entreprise ou d'un pays (au regard de la valeur des brevets). Les travaux des chercheurs visant à estimer ou déduire la valeur économique privée des brevets s'articulent autour de trois axes :

- Réalisation d'enquêtes auprès des inventeurs (ou titulaires) pour connaître la valeur économique de leur brevet (voir par exemple Scherer et al., 1999).

- Analyse des données tirées de la procédure de protection par brevet (délivrance du brevet ou rejet de la demande, citations, maintien en vigueur, étendue géographique de la protection, etc.).

- Estimation de la valeur des données financières (valeur de marché des entreprises, valeur des introductions en bourse, par exemple; voir Hall et al., 2005) ;

Selon la première méthode, les titulaires ou les inventeurs des brevets sont interrogés sur la valeur pécuniaire de leur brevet (c'est-à-dire le prix auquel ils seraient prêts à vendre l'invention, y compris les revenus générés par le brevet les années suivantes) ${ }^{2}$. On a montré que la distribution du rendement privé est assez biaisé avec un pic à zéro. Les brevets peuvent générer des rendements économiques de diverses façons : exploitation en interne ou par le biais de licences, "utilisation stratégique " (pour barrer la route à d'autres intervenants ou pour échanger des technologies), etc.

La deuxième approche met davantage en relief la valeur des brevets en utilisant des informations les concernant tirées principalement de données bibliographiques (figurant dans des publications, des rapports de recherche et d'examen, des oppositions, etc.) qui peuvent être corrélées avec la valeur des brevets. Certains de ces indicateur s'appuient sur le comportement observé des propriétaires de brevets pour estimer la valeur privée des brevets (fondée sur le renouvellement des brevets, le nombre de pays où le brevet est déposé, les décisions de vendre [céder] des brevets, etc.). D'autres indicateurs possibles de la valeur des brevets sont les citations en aval, le nombre de revendications et les oppositions et litiges.

La troisième approche est l'estimation économétrique de la contribution des catégories de brevets ou de portefeuilles de brevets à la performance économique des sociétés (par exemple, valeur en bourse, entreprises rejetons), en contrôlant leur stock de R-D et leur biens corporels. Par exemple, l'utilisation de la valeur marchande (tel le " $\mathrm{q}$ » de Tobin) ${ }^{3}$ pour estimer les rentes attribuées aux brevets suppose que le comportement des investisseurs 
peut révéler la valeur des brevets. Ces recherches ont uniformément trouvé une valeur marginale positive et significative pour les stocks de brevets et leur qualité (c'est-à-dire, le stock de brevets pondéré par les citations).

On trouvera dans ce chapitre les principales conclusions des travaux relevant de la deuxième approche. On cherchera à dégager de grands axes pour établir des statistiques sur les brevets qui tiennent compte de la dispersion de leur valeur et soient donc plus pertinentes sur le plan économique. Ces travaux en sont encore largement au stade de la recherche, et bon nombre des résultats présentés dans ce chapitre sont loin de faire l'unanimité parmi les experts. Il importe néanmoins de garder à l'esprit cette question de la valeur pour concevoir et interpréter les indicateurs relatifs aux brevets.

En utilisant des variables de substitution de la valeur des brevets, on peut calculer des indicateurs fondés sur les brevets qui soient moins affectés par la distribution asymétrique de cette valeur :

- Comptages pondérés : pondération du nombre de brevets par : le nombre de citations en aval; le nombre de membres de la famille, etc.

- Comptages de brevets déterminés (en éliminant les brevets à plus faible valeur ajoutée) : familles triadiques; brevets fréquemment cités (décile supérieur de la distribution); brevets délivrés (au lieu des demandes déposées); brevets maintenus en vigueur sur une période donnée (cinq ans, par exemple); etc.

\subsection{Citations en aval}

L'état de la technique antérieur à l'invention (brevets ou littérature hors brevets) cité dans les documents de brevets apporte des informations utiles sur la diffusion des technologies (voir le chapitre 6 sur l'utilisation des citations). On observe que le nombre de citations d'une demande de brevet dans des demandes ultérieures, qualifiées de " citations en aval ", est étroitement associé à la valeur économique des brevets (Scherer et al., 1999) et à la valeur sociale des inventions (Trajtenberg, 1990). Le nombre de citations en aval est l'un des indicateurs de la valeur les plus utilisés.

Deux grands arguments sont avancés pour justifier la validité des citations en aval comme indicateurs de la valeur des brevets : premièrement, elles révèlent l'existence d'efforts de recherche en aval, laissant entendre que des fonds sont investis dans le développement de la technologie en question (et qu'il existe un marché potentiel); et deuxièmement, le fait qu'un brevet donné ait été cité dans des demandes de brevet ultérieures donne à penser qu'il a été utilisé par des examinateurs pour limiter la portée de la protection revendiquée ensuite par un autre titulaire de brevet, au bénéfice de la société. En ce sens, les citations en aval traduisent à la fois la valeur privée et la valeur sociale des inventions. 
Quoi qu'il en soit, la principale difficulté inhérente au calcul du nombre de citations en aval est qu'elles apparaissent au fur et à mesure et souvent bien après que le brevet cité a fait l'objet d'une demande de dépôt, a été délivré ou est arrivé à son terme. Par souci de pertinence, il faut assurer l'actualité des indicateurs. On peut remédier à ce problème en dénombrant les citations des demandes de brevet dans un intervalle donné (dans les cinq premières années de leur publication, par exemple).

On dénombre couramment les citations en aval de la manière suivante : $C I T_{i, T}=\sum_{t=P_{i}}^{P_{i}+T} \sum_{j \in J(t)} C_{j, i}$ où $\operatorname{CIT}_{i, T}$ est le nombre de citations ultérieures d'une demande de brevet i publiée durant l'année $P_{i}$ au cours des années $T$ suivant sa publication. $C_{j, i}$ est une variable auxiliaire qui est égale à 1 si la demande $j$ cite la demande $i$, et 0 dans les autres cas. $J(t)$ est l'ensemble des demandes publiées durant l'année t. L'horizon retenu correspond souvent aux cinq années suivant la publication du brevet cité, puisqu'on a calculé pour les brevets déposés auprès de l'USPTO que plus de $50 \%$ des citations formulées pendant toute la durée de vie d'un brevet le sont pendant les cinq premières années ${ }^{4}$.

\subsection{Indicateurs fondés sur les informations sur la procédure et le comportement des demandeurs}

Les informations sur la valeur des brevets peuvent être obtenues à partir de données tirées du processus de demande de brevet (notamment sur le sort de la demande : retrait, refus ou délivrance du brevet) et relatives au comportement des demandeurs concernant le temps de survie des brevets (taux de maintien en vigueur) et la portée géographique de la protection (nombre de pays dans lesquels la protection par brevet a été demandée, nombre de familles de brevets internationales; voir le chapitre 3).

\subsubsection{Le sort de la demande de brevet}

On peut apprécier la qualité d'une invention en fonction de la délivrance ou non du brevet. Le brevet est attribué lorsque l'invention est officiellement reconnue comme remplissant les critères de brevetabilité : nouveauté, activité inventive (non-évidence) et applicabilité industrielle. Les brevets délivrés ont donc une valeur technologique et économique plus élevée que les demandes qui n'ont pas abouti ${ }^{5}$. Les demandes en instance peuvent avoir une certaine valeur sur le marché car elles sont la marque de droits potentiels susceptibles d'être mis en application à titre rétroactif après délivrance du brevet. Ainsi, la Convention sur le brevet européen prévoit qu'une demande publiée confère provisoirement au demandeur, dans tous les pays désignés, les mêmes droits que dans celui où le brevet a été délivré. 


\section{Tableau 8.1. Principaux indicateurs de la valeur des brevets analysés dans les publications}

\begin{tabular}{|c|c|c|}
\hline Indicateur & Argumentaire & Principales limites \\
\hline Brevets délivrés & $\begin{array}{l}\text { Protection juridique limitée si le brevet } \\
\text { n'est pas délivré; vérification effectuée } \\
\text { par des examinateurs. }\end{array}$ & $\begin{array}{l}\text { Ne sont guère instructifs (part importante : } \\
\text { quelque } 60 \% \text { des demandes de brevet sont } \\
\text { acceptées). USPTO : } 95 \% \text { des demandes } \\
\text { sont acceptées. }\end{array}$ \\
\hline Citations en aval & $\begin{array}{l}\text { Importance technologique des inventions; } \\
\text { impact sur les avancées technologiques } \\
\text { futures. }\end{array}$ & $\begin{array}{l}\text { Actualité (disponibilité dans le temps), } \\
\text { interprétation. }\end{array}$ \\
\hline $\begin{array}{l}\text { Taille de la famille } \\
\text { (nombre de pays) }\end{array}$ & $\begin{array}{l}\text { Coût de l'obtention d'une protection dans } \\
\text { différents pays. Témoigne du potentiel } \\
\text { commercial d'une invention. }\end{array}$ & $\begin{array}{l}\text { Problèmes de représentativité; une part } \\
\text { importante des demandes de brevet } \\
\text { sont internationales. }\end{array}$ \\
\hline Nombre d'inventeurs & $\begin{array}{l}\text { Représentatif du coût d'une invention } \\
\text { (coût de la recherche). }\end{array}$ & $\begin{array}{l}\text { Mesure approximative qui place les inventeurs } \\
\text { sur un pied d'égalité; nécessité de recueillir } \\
\text { des informations complémentaire sur } \\
\text { les inventeurs (carrières, protection par brevet, } \\
\text { etc.). }\end{array}$ \\
\hline Maintien en vigueur & $\begin{array}{l}\text { Coût de la conservation d'un brevet; } \\
\text { les taux de maintien en vigueur permettent } \\
\text { d'évaluer la distribution de la valeur. }\end{array}$ & $\begin{array}{l}\text { Actualité, influence des cycles de vie des } \\
\text { technologies, les taux de maintien en vigueur } \\
\text { varient selon les technologies (différence } \\
\text { de valeur). }\end{array}$ \\
\hline Opposition & $\begin{array}{l}\text { Valeur marchande d'un brevet. Coûts et risques } \\
\text { associés à des différends d'ordre juridique. }\end{array}$ & $\begin{array}{l}\text { Actualité, proportion très faible (environ } 5 \% \\
\text { pour l'OEB); comment détecter les accords } \\
\text { à l'amiable? }\end{array}$ \\
\hline Litiges & $\begin{array}{l}\text { Coûts et risques associés à des différends } \\
\text { d'ordre juridique. }\end{array}$ & $\begin{array}{l}\text { Actualité, proportion très faible, les règlements } \\
\text { à l'amiable sont fréquents, disponibilité } \\
\text { des données. }\end{array}$ \\
\hline $\begin{array}{l}\text { Valeur de marché de l'entreprise, } \\
\text { entreprise créée par essaimage, etc. }\end{array}$ & $\begin{array}{l}\text { Valeur des brevets intégrée en tant qu'actif } \\
\text { incorporel. }\end{array}$ & $\begin{array}{l}\text { Limitation à certains types d'entreprises } \\
\text { (marchés d'actions, etc.) }\end{array}$ \\
\hline $\begin{array}{l}\text { Valeur économique présentée } \\
\text { dans des enquêtes }\end{array}$ & $\begin{array}{l}\text { Valeur des brevets connue par les inventeurs } \\
\text { ou les dirigeants. }\end{array}$ & $\begin{array}{l}\text { Subjectivité, problèmes de sélection, } \\
\text { échantillons limités. }\end{array}$ \\
\hline
\end{tabular}

Source: Adapté de van Zeebroeck (2007).

Traditionnellement, l'USPTO publiait uniquement les brevets délivrés, et tous les brevets utilisés pour les indicateurs étaient donc similaires de ce point de vue. Néanmoins, la plupart des indicateurs reposant désormais sur les demandes, et non sur les brevets attribués, il faut prendre en compte cette source d'hétérogénéité : une partie des demandes comptabilisées ont donné lieu ou vont donner lieu à la délivrance d'un brevet, d'autres non. L'avantage du recours aux demandes est notable du point de vue de l'actualité, car le refus ou l'attribution d'un brevet intervient plusieurs années après la demande.

En analysant les caractéristiques de la demande de brevet (type de propriété, nombre d'inventeurs; coopération nationale et internationale, catégorie de technologie, date de priorité ou de demande, etc.), on peut identifier par des méthodes probabilistes les facteurs qui expliquent le refus, le retrait ou l'acceptation des demandes (voir Guellec et van Pottelsberghe, 2000) ${ }^{6}$. 


\subsubsection{Maintien en vigueur des brevets}

Les données sur le maintien en vigueur - ou renouvellement - des brevets et sur la taille de la famille (généralement définie dans les ouvrages économiques comme le nombre de pays dans lesquels une protection a été demandée; voir le chapitre 5 pour les définitions des familles de brevets) ont été amplement utilisées pour établir la valeur des brevets par déduction. Les études menées dans ce domaine partent du fait que pour un titulaire de brevet, conserver la protection qu'il confère pendant un délai et dans des pays supplémentaires coûte cher. On émet donc l'hypothèse que la valeur du maintien de la protection sur la durée et de l'élargissement de sa portée géographique est associée à l'importance économique de l'invention. On observe en toute logique une forte corrélation de ces deux types d'indicateurs.

Dans la plupart des systèmes de brevets, les titulaires doivent s'acquitter de taxes à intervalles réguliers pour que leurs brevets restent en vigueur. En règle générale, les taxes de maintien augmentent avec le temps et à chaque échéance, les titulaires doivent prendre la décision de demander ou non le renouvellement du brevet. S'ils s'abstiennent, le brevet est frappé de déchéance et l'invention tombe dans le domaine public. Les observations relatives à la proportion de brevets qui sont renouvelés à différents stades, ainsi que les grilles des taxes de maintien en vigueur correspondantes, fournissent des informations sur la distribution de la valeur des brevets et l'évolution de cette distribution pendant leur durée de vie (Griliches, 1990).

La logique sous-tendant cette approche repose sur des critères économiques. Les brevets sont renouvelés uniquement lorsque la valeur associée à cette opération (basée notamment sur les bénéfices attendus actualisés) est supérieure à son coût. Ainsi, lorsque les taxes de maintien en vigueur ne sont pas versées, les rendements escomptés (à l'avenir) sont inférieurs au seuil. Puisque les taxes augmentent au fil du temps dans la majorité des pays, les titulaires de brevets doivent étudier, pendant la période en cours, la rentabilité du renouvellement durant la période suivante (" option » de protection; Lanjouw et Schankerman, 1997) en fonction de son coût. Les titulaires ont habituellement du mal à évaluer les rendements qu'ils peuvent attendre d'un brevet. Il leur faut souvent du temps pour connaître le potentiel commercial des inventions, la décision de déposer un brevet étant fréquemment prise durant les premières phases du processus d'innovation ${ }^{7}$. Au Japon et aux États-Unis, les taxes de maintien en vigueur correspondant aux brevets délivrés aux universités et aux PME, ainsi qu'aux organismes publics peuvent bénéficier d'un régime préférentiel, et donc être moins élevées.

Les différentes enquêtes menées dans ce domaine ont confirmé que la distribution de la valeur des brevets était fortement asymétrique, avec une médiane nettement inférieure à la moyenne. Selon Pakes et Schankerman 
(1986), la moitié de la valeur estimée correspond à 5 \% environ de l'ensemble de la population des brevets analysée ${ }^{8}$. Dans une étude sur le renouvellement des brevets en Finlande et en Norvège, Pakes et Simpson (1989) observent que le taux de maintien en vigueur est particulièrement élevé dans l'industrie pharmaceutique et dans les secteurs du bois et du papier, suivis des inventions concernant les machines-outils, la chimie, l'agro-alimentaire et la métallurgie de base.

\section{Tableau 8.2. Part des pays dans le total des demandes de brevet en fonction de différents indicateurs}

Date de priorité 2000

\begin{tabular}{lcccc}
\hline & PCT & Triadiques & OEB & PCT les plus cités \\
\hline Allemagne & 0.13 & 0.13 & 0.19 & 0.17 \\
Canada & 0.02 & 0.01 & 0.01 & 0.01 \\
États-Unis & 0.40 & 0.33 & 0.27 & 0.31 \\
France & 0.05 & 0.05 & 0.06 & 0.06 \\
Japon & 0.10 & 0.31 & 0.19 & 0.17 \\
Pays-Bas & 0.03 & 0.02 & 0.03 & 0.03 \\
Royaume-Uni & 0.06 & 0.03 & 0.05 & 0.05 \\
Monde & 1.00 & 1.00 & 1.00 & 1.00
\end{tabular}

Note : Critères de comptage : pays de résidence du ou des inventeurs et date de priorité. Demandes de brevet PCT en phase internationale, désignant l'OEB; OEB : demandes OEB directes, plus Euro-PCT en phase régionale; et famille de brevets triadiques : sous-ensemble des brevets déposés conjointement auprès de l'OEB, du JPO et délivrés par l'USPTO (protégeant le même groupe d'inventions; date de priorité identique). PCT les plus cités : demandes de brevet PCT en phase internationale (désignant l'OEB) qui sont parmi les plus fréquemment citées (décile supérieur). Les données sur les familles de brevets triadiques sont principalement tirées de PATSTAT.

L'approche fondée sur le maintien en vigueur des brevets se heurte à plusieurs limites. Les résultats de ces études reposent sur des hypothèses relatives à la forme fonctionnelle des relations et sur la valeur non observée des brevets à plus forte valeur ajoutée - ceux qui sont renouvelés jusqu'à extinction de la durée légale ("sans restriction "). Dans certains cas, les abandons ne témoignent pas d'une faible valeur des brevets, mais plutôt d'une modification de la stratégie de l'entreprise, liée par exemple à un choc externe. Dans les technologies qui évoluent à un rythme soutenu, de nombreuses inventions présentent une forte valeur ajoutée lors de leur introduction, mais deviennent rapidement obsolètes. Des facteurs exogènes peuvent également influer sur la décision de renouveler un brevet. Ainsi, Schankerman (1998) observe des éléments attestant des chocs pétroliers dans les données françaises de maintien en vigueur des brevets ${ }^{9}$. Enfin, le profil temporel du chiffre d'affaires généré peut dépendre du domaine technique et d'autres caractéristiques des inventions; celles-ci deviennent obsolètes plus rapidement dans l'électronique que dans les produits pharmaceutiques. 


\subsubsection{Taille de la famille de brevets}

La valeur des brevets est également associée à la portée géographique de la protection qu'ils confèrent, c'est-à-dire le nombre de pays dans lesquels la délivrance a été demandée (voir le chapitre 4 pour les définitions des familles de brevets). Le fait de demander une protection par brevet à l'étranger témoigne déjà d'une valeur économique, une telle décision reflétant la volonté du propriétaire d'assumer les coûts d'une protection internationale. La logique sousjacente est étroitement liée à la décision de renouveler un brevet; assurer la validité d'un brevet dans plusieurs pays (ce qui suppose de déposer une demande directement ou indirectement auprès de bureaux régionaux ou internationaux) et maintenir cette protection en vigueur coûtent cher (Putnam, 1996).

À l'inverse des données sur les renouvellements qui sont disponibles sur la durée (ou des statistiques sur les citations en aval, voir chapitre 6), le nombre de pays dans lesquels la protection est demandée est disponible plus tôt (droit de priorité pendant un an selon la Convention de Paris). C'est un avantage de cette source d'informations, qui permet d'établir des indicateurs plus en amont de la durée de vie d'une demande de brevet.

La portée géographique de la protection, mesurée par les brevets internationaux délivrés pour une invention donnée, correspond à la couverture commerciale d'une invention : plus le nombre de pays dans lesquels la protection est demandée est élevé, plus le potentiel de ventes et de bénéfices est important ${ }^{10}$. Des données concordantes montrent que la taille de la famille reflète la valeur économique. Ainsi, Lanjouw et Schankerman (2004) notent un lien positif très net entre un indice de qualité des brevets et la taille de la famille (sur un échantillon de brevets américains). Guellec et van Pottelsberghe de la Potterie (2000) font état d'une relation positive entre la taille de la famille et la probabilité qu'un brevet européen soit délivré. Harhoff et al. (2002) démontrent que les brevets appartenant à une vaste famille de brevets internationaux sont plus étroitement liés à une valeur économique. Dans le groupe des produits pharmaceutiques et chimiques, cet indicateur présente le coefficient le plus élevé de tous les ensembles de résultats propres aux technologies.

Dans le cas du système de brevets européen, la liste des pays contractants de la CBE (Convention sur le brevet européen) dans lesquels la protection est demandée figure dans la demande. Le paiement de la taxe de dépôt à l'OEB repose sur cette liste, bien que ce lien se soit atténué avec le temps. Pour les demandes européennes et internationales déposées le $1^{\mathrm{er}}$ juillet 1999 ou après cette date auprès de l'OEB, la taxe de désignation est réputée versée pour tous les États contractants lorsqu'elle a été acquittée auprès de sept pays au moins. En fait, en vertu de la CBE de 2000, les demandes sont réputées désigner l'ensemble des États contractants par le biais d'une taxe de désignation forfaitaire unique (voir encadré 8.1). À compter du mois d'avril 2009, les 
demandes de brevet européen désignent l'ensemble des États contractants, à l'image de la procédure $\mathrm{PCT}^{11}$.

\section{Encadré 8.1. Réformes concernant la désignation des États}

Pour calculer des indicateurs relatifs à l'ampleur de la protection géographique à partir des États désignés dans les demandes OEB et PCT, il importe de savoir que ces indicateurs ne seront plus pertinents en tant qu'indicateurs de la couverture commerciale, en raison de la convergence des procédures relevant du PCT et de l'OEB vers une désignation automatique (de tous les États contractants) avec une taxe forfaitaire unique. Il faut donc connaître la date d'entrée en vigueur de ces réformes lorsqu'on travaille avec des séries temporelles de données sur les brevets :

\section{La désignation dans le cadre du Traité de coopération en matière de brevets}

Pour les demandes internationales dont la date de dépôt international est équivalente ou postérieure au $1^{\mathrm{er}}$ octobre 1998 : une taxe de désignation est fixée par pays ou par région pour les 11 premiers bureaux nationaux ou régionaux. Aucune taxe supplémentaire n'est prélevée pour les désignations dépassant ces 11 bureaux. À compter du mois d'avril 2009, les demandes de brevet européen désignent tous les États contractants, à l'image de la procédure PCT.

Pour les demandes internationales dont la date de dépôt international est équivalente ou postérieure au $1^{\mathrm{er}}$ janvier 2004 : le dépôt d'une requête internationale vaut désignation de tous les États contractants liés par le Traité à la date du dépôt international.

\section{La désignation dans le cadre de l'OEB}

Depuis le $1^{\text {er }}$ juillet 1999 et pour toutes les demandes européennes et internationales entrant dans la phase régionale (déposées à cette date ou ultérieurement), chaque État contractant désigné donne lieu à une taxe de désignation à concurrence de sept États désignés. Aucune taxe supplémentaire n'est prélevée en cas de désignation de plus de sept bureaux.

À compter du $1^{\mathrm{er}}$ avril 2009 : désignation automatique des États contractants et taxe unique. Une taxe forfaitaire est prélevée quel que soit le nombre d'États contractants désignés. Cette décision s'applique aux demandes de brevet européen déposées le $1^{\mathrm{er}}$ avril 2009 ou après cette date, ainsi qu'aux demandes internationales entrant dans la phase régionale à ou après cette date.

Après sa délivrance, la taille de la famille d'un brevet européen peut être quantifiée à l'aide du nombre d'États contractants de la CBE dans lesquels le brevet a effectivement été validé après attribution. La taille de la famille CBE peut 
naturellement diminuer avec le temps, à mesure que des brevets sont abandonnés dans divers pays, d'où la nécessité d'observer la portée géographique à différents périodes. Les informations sur le maintien en vigueur et la portée géographique de la protection peuvent être utilisées pour élaborer des indicateurs

\section{Encadré 8.2. Un indicateur mixte (protection européenne) : l'indice de la portée annuelle}

On peut se servir des données sur l'interaction du maintien en vigueur et de la portée géographique de la protection d'un brevet pour élaborer un indice plus fin tenant compte de l'évolution de la protection géographique dans le temps (les brevets pouvant être chaque année frappés de déchéance dans certains pays. Cet indicateur traduit à la fois l'âge du brevet et la taille de la famille européenne (van Pottelsberghe et van Zeebroeck, 2007) :

$$
\mathrm{SY}_{\mathrm{CT}, \mathrm{i}}=\frac{\sum_{t=1}^{T} \sum_{c=1}^{C} G_{i}(c, t)}{C X T}
$$

Soit $\mathrm{SY}_{\mathrm{CT}, \mathrm{i}}$ l'indice de la portée annuelle (SY) d'un brevet $i$ dans les pays $C$ et sur $\mathrm{T}$ années de maintien, et $\mathrm{Gi}(\mathrm{C}, \mathrm{t})$ une variable égale à 1 si le brevet $i$ accordé est en vigueur dans le pays ct années après la date de dépôt et 0 dans le cas contraire. La base de l'indice est sa valeur maximum qui représente $T$ années de maintien dans les pays $C$. Ainsi, l'indicateur donne pour chaque année de vie d'un brevet le nombre de pays dans lesquels il est en vigueur en Europe. Pour assurer la comparabilité de l'indicateur sur la durée et sa disponibilité dans les 10 années suivant la date de dépôt, l'indicateur proposé par les auteurs est calculé sur 10 ans pour 10 pays. On élimine ainsi la distorsion institutionnelle de la taille des familles (élargissement institutionnel de la CBE, de 10 pays en 1977 à 32 en 2007).

On peut élargir cet indicateur en pondérant la validation obtenue dans certains pays par leur importance économique, notamment par leur PIB. Il convient de noter qu'en tant que tel, l'indice SY des demandes rejetées est nécessairement égal à zéro, les brevets ne pouvant être validés dans les États contractants de la CBE qu'après avoir été attribués par l'OEB. Une version provisoire de l'indice SY a été proposée, tenant compte de la durée de la procédure de délivrance (nombre d'années durant lesquelles la demande a été renouvelée). Voir van Pottelsberghe et van Zeebroeck (2007) pour de plus amples détails sur cette formulation.

plus fins tenant compte de l'évolution de la protection dans le temps et dans différents pays (car les brevets peuvent être chaque année frappés de déchéance dans certains pays, voir encadré 8.2). 


\subsection{Autres indicateurs}

\subsubsection{Le nombre de revendications}

La portée d'un brevet est un indicateur important de sa valeur économique, car elle délimite juridiquement la protection accordée et, partant, la valeur attribuée au brevet sur le marché. Une portée plus large renvoie à un espace technologique plus vaste dont les autres sont exclus.

Cela étant, la " portée " ou " étendue " d'un brevet est difficile à mesurer. La portée d'un brevet apparaît dans les revendications, mais elle apparaît concrètement dans les citations de brevets antérieurs - qui définissent les frontières juridiques du brevet par rapport à l'état antérieur de la technique ${ }^{12}$. Plusieurs économistes ont utilisé le nombre de revendications pour apprécier la portée juridique des brevets. Certains ont fait valoir que chaque brevet représentant un ensemble de composants d'innovation, correspondant chacun à une revendication, le nombre de revendications pouvait donner une idée de la valeur du brevet dans son ensemble. Cependant, la tendance de certains demandeurs à "gonfler " le nombre de revendications à des fins stratégiques brouille quelque peu les liens entre la portée et le nombre de revendications. De plus, il faut préciser que pour les brevets délivrés, les revendications qui apparaissent sont celles qui ont été retenues après examen.

Les analyses empiriques réalisées sur ce point sont rares, mais particulièrement concluantes. Dans leur modèle multifactoriel de la qualité des brevets destiné à analyser la productivité de la recherche aux États-Unis, Lanjouw et Schankerman (2004) observent que le nombre de revendications est le principal indicateur de la qualité des brevets dans six des sept domaines technologiques. Ils notent également que la probabilité qu'un brevet donne lieu à un litige, ce qui est un signe de sa portée, s'accroît parallèlement au nombre de revendications qu'il contient (Lanjouw et Schankerman, 1997).

\subsubsection{Le nombre de classes techniques}

Le nombre de classes techniques (correspondant au nombre de classes CIB) attribuées à une demande de brevet a également été utilisé pour évaluer la portée, et donc la valeur, d'un brevet. Cette approche a été proposée par Lerner (1994) dans une étude sur la valeur marchande des brevets de biotechnologie en tant que mesure de la valeur du portefeuille de brevets. Lerner a observé une corrélation positive et notable entre la valeur de l'entreprise sur le marché et la portée moyenne de ses brevets.

Toutefois, rares sont les éléments qui corroborent la corrélation entre le nombre de classes et la valeur d'un brevet. Lanjouw et Schankerman (1997) notent un effet légèrement positif du nombre de classes CIB sur la probabilité de survenance de procès en violation de brevets aux États-Unis. À partir des 
informations tirées d'une enquête sur la valeur économique des brevets perçue par les inventeurs allemands, Harhoff et al. (2002) observent que le nombre de classes CIB à quatre chiffres n'est révélateur de la valeur des brevets dans aucun des domaines technologiques analysés ${ }^{13}$.

\subsubsection{Le nombre d'inventeurs mentionnés dans un brevet}

Plusieurs études économiques ont associé le nombre d'inventeurs répertoriés dans un brevet à sa valeur, à la fois économique et technologique. Le nombre d'inventeurs peut être représentatif du coût de la recherche ayant abouti à l'invention, lui-même statistiquement relié à la valeur technique de l'invention: plus les ressources sont importantes, plus le projet est consommateur de recherche et coûteux (Guellec et van Pottelsberghe, 2001; Gambardella et al., 2005).

\subsubsection{Opposition et litiges}

Certains bureaux des brevets offrent à des tiers la possibilité de faire opposition à des brevets délivrés lorsqu'ils jugent qu'ils ne sont pas valables. Un tel acte pouvant être coûteux, on peut en déduire que seuls les brevets qui sont dommageables à la concurrence, et qui ont une certaine valeur économique, seront concernés. On peut donc interpréter cette opposition comme un signe de la valeur du brevet. De plus, ceux qui "survivent " à une opposition sont alors considérés comme des brevets dont la résistance est avérée et qui présentent des perspectives extrêmement rentables pour leur titulaire.

Rares sont les brevets qui font l'objet d'une opposition. En 2006, le taux d'opposition ressortait aux alentours de $5.4 \%$ (des oppositions ont été formées contre 2990 brevets) à l'OEB. Parmi ceux qui ont fait l'objet d'une opposition à l'OEB, un tiers environ sont révoqués, un tiers sont maintenus tels que délivrés et un tiers maintenus sous une forme modifiée. À l'USPTO, les parties intéressées souhaitant contester un brevet américain après sa délivrance ont deux solutions : i) le contester devant un tribunal fédéral; ou ii) demander qu'il soit " réexaminé » par l'USPTO. Le taux d'opposition de l'OEB est nettement supérieur au taux de réexamen de l'USPTO, toutes classes technologiques confondues (Merges, 1999; Graham et al., 2002). De 1981 à 1998, le taux de réexamen de l'USPTO s'établissait à $0.3 \%$ (des brevets délivrés), alors qu'à l'OEB, le taux d'opposition s'inscrivait en moyenne à 8.6 \% des brevets attribués sur la même période. En termes absolus, toutefois, le nombre des litiges portant sur des brevets a sensiblement progressé aux États-Unis de 1985 à 2000, bien que le taux de litiges rapporté au nombre de brevets attribués soit demeuré constant (Graham et al., 2002).

Certains auteurs observent que les brevets ayant fait l'objet d'une opposition ou d'un litige présentent une valeur ajoutée supérieure à la moyenne. Harhoff et al. (2002) constatent que le fait d'avoir résisté à une opposition (dans le système 
de brevet allemand) est un moyen particulièrement fiable d'estimer la valeur des brevets $^{14}$. Ils expliquent que les droits de brevet les plus résistants survivent à un processus de sélection à deux niveaux (brevet délivré et survie à l'opposition), qui constitue un indicateur particulièrement fiable de leur qualité. Selon Lanjouw et Schankerman (1998), les brevets qui sont contestés ont des caractéristiques particulières. Par rapport à un échantillon aléatoire de brevets américains concernant les mêmes cohortes et domaines technologiques, les auteurs observent que les brevets à plus forte valeur ajoutée et ceux qui sont détenus par des résidents sont bien plus sujets à des litiges. Les brevets appartenant à des particuliers risquent au moins autant de faire l'objet d'un procès que ceux des entreprises et les litiges sont particulièrement courants dans les nouveaux domaines technologiques.

\section{Notes}

1. Des inventions dont la valeur technique est élevée peuvent présenter une forte appropriabilité (parce qu'il est facile de contourner les brevets dans le domaine technologique auquel appartient l'invention, par exemple). Des inventions de faible valeur technique peuvent générer une valeur économique élevée, notamment lorsque l'inventeur est déjà, pour des raisons diverses, en situation de monopole sur le marché.

2. Cette approche a le mérite de recueillir des données directement à la source. Elle peut néanmoins être faussée, car l'inventeur ou le propriétaire du brevet peut ne pas avoir ou ne pas être disposé à communiquer des informations exactes.

3. Le « q » de Tobin est défini comme le rapport de la valeur sur le marché des actifs d'une entreprise au coût de remplacement de l'entreprise, qui est généralement mesuré par la valeur de remplacement des biens corporels de l'entreprise.

4. Selon Lanjouw et Schankerman (1998), limiter à cinq ans la période suivant la délivrance du brevet est suffisant pour obtenir des mesures valables de "l'importance » d'un brevet en se fondant sur les citations « en aval ".

5. Toutefois, la délivrance d'un brevet n'est pas toujours un bon indicateur. Ainsi, une meilleure connaissance du dispositif européen peut faire grimper les taux de délivrance dans les pays contractants de la CBE par rapport à ceux enregistrés par les demandeurs américains qui déposent une demande auprès de l'OEB (Hinze et Schmoch, 2004).

6. Selon certains travaux (Reitzig, 2004; Burke et Reitzig, 2007, par exemple), une "requête en examen accéléré " auprès de l'OEB (des procédures analogues existent pour le JPO et l'USPTO) peut correspondre à des inventions à forte valeur ajoutée que le propriétaire souhaite protéger le plus rapidement possible.

7. Un faible nombre seulement de brevets sont maintenus en vigueur jusqu'à leur terme. Ainsi, Pakes et Schankerman (1986) ont montré que $10 \%$ seulement de l'ensemble des brevets vont au-delà de la période de maintien en vigueur. Selon Lemley (2001), qui utilise les données relatives au maintien en vigueur des brevets américains en 1998, près des deux tiers de tous les brevets délivrés aux États-Unis s'éteignent avant leur échéance, faute de versement des taxes de maintien en 
vigueur, et quasiment la moitié des brevets sont abandonnés avant que la moitié de leur durée de vie potentielle se soit écoulée.

8. Pakes (1986) explique que le flux de revenus évolue différemment au cours du cycle de la protection et les premières années se caractérisent souvent par une forte incertitude économique. À mesure que la rentabilité de l'invention apparaît plus clairement, l'incertitude s'estompe progressivement lorsque le brevet atteint un certain seuil - entre quatre et cinq ans; voir Pakes (1986) et Lanjouw (1998).

9. Dans l'industrie pharmaceutique, les taux de maintien en vigueur peuvent être intrinsèquement plus élevés que dans d'autres secteurs en raison de facteurs institutionnels comme la longueur des délais réglementaires existant entre le développement des médicaments et leur mise sur le marché.

10. Les mesures de la taille de la famille (ou nombre des inventeurs) dépend du pays d'origine; ainsi, la taille de la famille des pays européens est toujours supérieure à celle des demandes japonaises (compte tenu du nombre élevé des pays limitrophes en Europe).

11. Les demandes déposées conformément au PCT peuvent être considérées comme un indicateur des inventions dont le potentiel commercial attendu est plus élevé. Cet indicateur peut se décomposer en PCT I et PCT II. On peut obtenir de plus amples informations en s'intéressant à l'intervalle écoulé entre deux étapes, en cherchant par exemple à savoir si le délai courant entre la date de dépôt et le début de la phase régionale est inférieur ou égal à 20 mois (PCT I) ou supérieur à 20 mois (PCT II). On peut arguer que plus le demandeur est prêt à payer pour pouvoir reporter des décisions coûteuses durant la demande, plus il a d'incertitudes quant à la valeur commerciale du brevet concerné (voir Burke et Reitzig, 2007).

12. Comme l'ont montré les entretiens menés avec des juristes spécialistes des brevets et avec des examinateurs, une demande de brevet cherchant à obtenir une protection étendue peut inciter l'examinateur à délimiter les revendications du brevet en faisant davantage référence aux ouvrages publiés s'y rapportant. Dans ce sens, les citations antérieures traduisent également la portée d'un brevet, ainsi que l'existence d'un objet susceptible de la limiter (Harhoff et al., 2002).

13. Les auteurs expliquent que la différence de résultats peut s'expliquer par l'utilisation de brevets couvrant un large éventail de domaines techniques, tandis que l'étude de Lerner porte principalement sur les brevets biotechnologiques. Ils soulignent également qu'il peut aussi y avoir des différences notables dans l'application de la classification CIB par les bureaux des brevets allemand et américain.

14. Ils notent qu'un brevet qui a résisté à une opposition ("Einspruchsverfahren ") en Allemagne a une valeur nettement plus élevée - d'après la valeur pécuniaire des inventions estimée par les inventeurs (par un facteur de 11.2) - qu'un brevet qui n'a jamais été contesté. De plus, si l'opposition contre le brevet a été formée selon la procédure en annulation, qui est plus onéreuse, sa valeur est bien plus élevée que celle des droits de brevet non contestés, dans ce cas par un facteur de 42.6 .

\section{Références}

Burke, P. et M. Reitzig (2007), " Measuring Patent Assessment Quality - Analyzing the Degree and Kind of (In)consistency in Patent Offices' Decision Making ", Research Policy, vol. 36, pp. 1404-1430.

Gambardella, A., D. Harhoff et B. Verspagen (2005), « The Value of Patents ", mimeo. 
Graham, S.J.H., B.H. Hall, D. Harhoff et D.C. Mowery (2002), « Post-Issue Patent 'Quality Control': A Comparative Study of US Patent Re-examinations and European Patent Oppositions ", NBER Working Paper 8807, National Bureau of Economic Research, Inc.

Griliches, Z. (1990), "Patent Statistics as Economic Indicators: A Survey », Journal of Economic Literature, 28, pp. 1661-1707.

Guellec, D. et B. van Pottelsberghe (2000), "Applications, Grants and the Value of Patents ", Economic Letters 69 (1), pp.109-114.

Guellec, D. et B. van Pottelsberghe (2001), «The Internationalisation of Technology Analysed with Patent Data ", Research Policy, 30 (8), pp. 1256-1266.

Hall, B.H., A. Jaffe et M. Trajtenberg (2005), « Market Value and Patent Citations », Rand Journal of Economics 36, printemps.

Harhoff, D., F.M. Scherer et K. Vopel (2002), Citations, Family Size, Opposition and the Value of Patent Rights, Research Policy, Elsevier, 32 (8), pp. 1343-1363.

Hinze, S. et U. Schmoch (2004), « Opening the Black Box. Analytical approaches and their impact on the outcome of statistical patent analyses » in W. Glänzel, H. Moed and U. Schmoch (éd.) (2004): Handbook of Quantitative Science and Technology Research: The Use of Publication and Patent Statistics in Studies on R\&D Systems, Kluwer Academic Publishers, Dordrecht/Boston/Londres, pp. 215-235.

Lanjouw, J. et M. Schankerman (2004), « Patent Quality and Research Productivity: Measuring Innovation with Multiple Indicators ", The Economic Journal, 114, pp. 441-465.

Lanjouw, J.O. (1998), "Patent Protection in the Shadow of Infringement: Simulation Estimations of Patent Value ", The Review of Economic Studies, vol. 65, pp.671-710.

Lanjouw, J.O., A. Pakes et J. Putnam (1998), « How to Count Patents and Value Intellectual Property: Uses of Patent Renewal and Application Data ", The Journal of Industrial Economics, vol. XLVI, no. 4, décembre, pp. 405-433.

Lanjouw, J.O. et M. Schankerman (1997), «Stylised Facts of Patent Litigation: Value, Scope and Ownership », NBER working paper series, NBER, Cambridge, MA.

Lanjouw, J.O. et M. Schankerman (1998), « Patent Suits: Do They Distort Research Incentives? ", Centre for Economic Policy Research, Londres, CEPR working paper series, $\mathrm{n}^{\circ} 2042$.

Lemley, M.A. (2001), « Rational Ignorance at the Patent Office », Northwestern University LawReview 95:4, pp. 1497-1532.

Lerner, J., (1994), « The Importance of Patent Scope: An Empirical Analysis », RAND Journal of Economics 25 (2), pp. 319-333.

Merges, R. P. (1999), "As Many as Six Impossible Patents Before Breakfast: Property Rights for Business Concepts and Patent System Reform ", Berkeley High Technology Law Journal 14, pp. 577-615.

Pakes, A. (1986), « Patents as Options: Some Estimates of the Value of Holding European Patent Stocks », Econometrics 54 (4), pp. 755-784.

Pakes, A. et M. Schankerman (1986), «Estimates of the Value of Patent Rights in European Countries During the Post-1950 Period ", Economic Journal, December, pp. 1052-1076.

Pakes, A. et M. Simpson (1989), « Patent Renewal Data », NBER Reprints 1265, National Bureau of Economic Research, Inc. 
van Pottelsberghe, B. et N. van Zeebroeck (2007), « A Brief History of Space and Time: The Cope-Year Index as a Patent Value Indicator Based on Families and Renewals ", CEPR Discussion Papers 6321.

Putnam, J. (1996), "The Value of International Patent Rights ", Ph.D. thesis, Yale University.

Reitzig, M. (2004), « Improving Patent Valuations for Management Purposes: Validating New Indicators by Analyzing Application Rationales ", Research Policy, vol. 33 (6-7), pp.939-957.

Schankerman, M. (1998), « How Valuable Is Patent Protection? Estimates by Technology Field ", RAND Journal of Economics, vol. 29 (1), 77-107, The RAND Corporation.

Trajtenberg, M. (1990), «A Penny for Your Quotes: Patent Citations and the Value of Innovation ", RAND Journal of Economics, 21 (1), pp.172-187.

van Zeebroeck, N. (2007), « The Puzzle of Patent Value Indicators », CEB Working Papers 07-023.RS, Université Libre de Bruxelles, Solvay Business School, Centre Emile Bernheim (CEB). 



\section{Glossaire}

Activité inventive : à l'OEB et au JPO, une invention est considérée comme impliquant une activité inventive lorsqu'elle n'est pas évidente pour une personne possédant des compétences ordinaires dans la technique. L'activité inventive est l'un des critères (aux côtés, notamment, de la nouveauté et de l'applicabilité industrielle) qu'il convient de remplir pour obtenir un brevet. Voir également « non-évidence » (USPTO).

Administration chargée de la recherche internationale : administration chargée de réaliser la recherche internationale pour une demande PCT. Il peut s'agir d'un bureau national (Australie, Autriche, Canada, Chine, Corée, Espagne, États-Unis, Fédération de Russie, Finlande, Japon, Suède) ou d'une organisation intergouvernementale (OEB) (article 16 du PCT, article 154 de la CBE).

Attributaire : aux États-Unis, personne(s) ou entreprise à laquelle l'intégralité ou une partie des droits conférés par un brevet sont juridiquement transférés par l'inventeur (équivalent de « demandeur » dans ce contexte).

Brevet : un brevet est un droit de propriété intellectuelle conféré par des instances compétentes, en vertu duquel son propriétaire a le droit juridique d'empêcher des tiers d'utiliser, de vendre, d'importer, etc. l'invention revendiquée dans le ou les pays concernés, pendant une durée ne pouvant excéder 20 ans à compter de la date de dépôt. Les brevets sont délivrés à l'inventeur aux États-Unis et aux entreprises, particuliers ou autres entités à condition que l'invention satisfasse aux critères de brevetabilité : nouveauté, non-évidence et applicabilité industrielle. Aux États-Unis, le terme « utility patent » est utilisé.

Brevet européen : un brevet européen peut être obtenu pour tous les pays contractants de la CBE en effectuant un dépôt unique auprès de l'OEB dans l'une des trois langues officielles (allemand, anglais ou français). Les brevets européens délivrés par l'OEB confèrent les mêmes droits juridiques et sont soumis au même régime que les brevets nationaux (attribués par le bureau national des brevets). Il convient de noter qu'un brevet européen délivré correspond à un ensemble de brevets nationaux, qui doivent être validés par chaque bureau national pour être valables dans les États membres. Le processus de validation peut prévoir la production d'une traduction du document brevet, le versement d'une taxe et d'autres formalités à accomplir auprès du bureau 
national (en d'autres termes, lorsqu'un brevet européen est délivré, la compétence est transférée aux bureaux nationaux).

Citations : références à l'état antérieur de la technique dans les documents de brevet. Les citations peuvent être le fait de l'examinateur ou du demandeur. Elles comprennent une liste de références considérées comme l'état de la technique se rapportant à l'invention et peuvent avoir joué dans la définition de la portée des revendications figurant dans la demande. Il est possible de faire référence à d'autres brevets, à des revues techniques, à des manuels, à des guides ou à d'autres sources. USPTO : les demandeurs auprès de l'USPTO sont tenus de présenter l'état antérieur de la technique dont ils ont connaissance et qui est déterminant pour la brevetabilité; OEB : les demandeurs ne sont tenus à aucune obligation de ce type; JPO : l'obligation de faire état d'informations relatives à des documents présentant l'état antérieur de la technique a été introduite le $1^{\mathrm{er}}$ septembre 2002 et est pleinement entrée en vigueur le $1^{\mathrm{er}}$ mai 2006.

Classification internationale des brevets (CIB) : la CIB repose sur un traité international multilatéral administré par l'OMPI. Internationalement reconnue, la CIB propose une classification commune des brevets en fonction de groupes technologiques. Il s'agit d'un système hiérarchique qui organise le champ de la technique en huit sections, subdivisées en classes, sous-classes et groupes. La CIB est révisée à intervalle régulier afin d'améliorer le système et de tenir compte de l'évolution de la technique. La $8^{\mathrm{e}}$ édition de la CIB est entrée en vigueur le $1^{\text {er }}$ janvier 2006.

Continuation(s) (USPTO) : deuxième demande ou demande ultérieure déposée pour la même invention revendiquée dans une précédente demande non provisoire alors que la première demande n'a été ni abandonné ni acceptée. Les continuations doivent porter sur la même invention que la demande d'origine pour conserver le bénéfice de la date de dépôt initiale. Lors de la demande, les revendications sont souvent les mêmes, mais peuvent évoluer au cours de la procédure, de sorte qu'elles ne sont pas exactement les mêmes sans être distinctes du point de vue de la brevetabilité. Cela concerne trois types de demandes: la division, la continuation et la continuation partielle (continuation-in-part).

Convention de Paris : la Convention de Paris pour la protection de la propriété industrielle, qui date de 1883, est plus connue sous le nom de Convention de Paris. Elle instaure le système des droits de priorité, en vertu desquels les demandeurs disposent d'un délai de 12 mois à compter de leur première demande (habituellement déposée dans leur pays) pour effectuer des demandes ultérieures dans chaque pays signataire et revendiquer la date de priorité originale. On dénombre 172 pays parties à ce traité (mars 2008).

Convention sur le brevet européen (CBE) : signée à Munich en 1973, la Convention sur la délivrance de brevets européens est entrée en vigueur en 1977. 
Il s'agit d'un traité multilatéral instituant l'Organisation européenne des brevets et prévoyant un système juridique autonome dans le cadre duquel les brevets européens sont attribués. La CBE constitue un cadre juridique de délivrance des brevets européens, à travers une procédure unique, harmonisée, devant l'Office européen des brevets. Elle donne aux demandeurs la possibilité d'obtenir, au moyen d'une procédure unique, un brevet dans une partie ou dans la totalité des États contractants. On recensait en janvier 200834 États contractants de la CBE. De plus, des accords d'extension existant dans cinq pays offrent la possibilité d'étendre des brevets européens à ces pays sur demande. Les États contractants de la CBE sont l'Allemagne, l'Autriche, la Belgique, la Bulgarie, Chypre, la Croatie, le Danemark, l'Espagne, l'Estonie, la Finlande, la France, la Grèce, la Hongrie, l'Irlande, l'Islande, l'Italie, la Lettonie, le Liechtenstein, la Lituanie, le Luxembourg, Malte, Monaco, la Norvège, les Pays-Bas, la Pologne, le Portugal, la République tchèque, la Roumanie, le Royaume-Uni, la Slovaquie, la Slovénie, la Suède, la Suisse et la Turquie. Les États autorisant l'extension dans le cadre de la CBE sont l'Albanie, la Bosnie-Herzégovine, la Croatie, l'ex-République yougoslave de Macédoine et la Serbie.

Date de délivrance : date à laquelle le bureau des brevets octroie un brevet au demandeur.

Date de dépôt : date à laquelle le bureau des brevets reçoit la demande complète de brevet. Une référence unique est attribuée à une demande de brevet lors du dépôt.

Date de priorité : la date de priorité est la date du premier dépôt d'une demande de brevet effectué, où que ce soit dans le monde (généralement auprès du bureau des brevets du pays du demandeur), en vue de protéger une invention. La date de priorité sert à déterminer la nouveauté de l'invention, de sorte qu'il s'agit d'un concept important dans les procédures relatives aux brevets. Parmi les données liées à la procédure, la date de priorité peut être considérée comme la date la plus proche de celle de l'invention. Aux États-Unis, la date de conception entre en jeu en cas de recoupement.

Déchéance : date à laquelle un brevet n'est plus valable dans un pays ou un système en raison du non-versement de la taxe de maintien en vigueur (ou de renouvellement). Souvent, les droits y afférant peuvent être restaurés durant un délai limité.

Délai de traitement : durée d'un processus au cours de la procédure relative aux brevets (recherche, examen, délivrance, et opposition et recours possibles, notamment).

Délivrance : une demande de brevet ne confère pas automatiquement au demandeur un droit temporaire contre toute violation. Un brevet doit être délivré pour être valable et opposable en cas de violation. 
Demande en instance : demande de brevet déposée auprès du bureau des brevets, mais n'ayant fait l'objet d'aucune décision visant à l'accepter ou à la rejeter.

Demande internationale de brevet : voir "demande PCT ». Une demande de brevet déposée conformément au Traité de coopération en matière de brevets (Patent Cooperation Treaty, PCT) est couramment qualifiée de "demande internationale de brevet ». Toutefois, une telle demande ne donne pas lieu à la délivrance d'un « brevet international » (c'est-à-dire qu'à l'heure actuelle, il n'y a aucun système de brevet mondial qui permette de délivrer et de faire respecter des brevets internationaux). La décision d'accepter ou de rejeter une demande de brevet conforme au PCT revient aux bureaux des brevets nationaux ou régionaux (comme l'OEB).

Demande nationale : demande de brevet déposée auprès d'un bureau national conformément à une procédure nationale.

Demandeur : titulaire des droits et obligations juridiques attachés à une demande de brevet. C'est le plus souvent une entreprise, une université ou un particulier.

Division : si le bureau des brevets décide qu'une demande couvre un domaine trop vaste pour être considérée comme un brevet unique, elle est divisée en une ou plusieurs demandes divisionnaires, auxquelles le demandeur peut donner suite ou pas. Une division peut également intervenir sur requête du demandeur.

Droits de priorité : voir " Convention de Paris ".

Droits de propriété intellectuelle (DPI) : droits exclusifs juridiques associés à une œuvre de création, des symboles commerciaux ou des inventions. On peut classer les DPI en quatre grandes catégories : les brevets, les marques, les dessins et les droits d'auteur.

Équivalent : brevet protégeant la même invention et ayant la même demande de priorité qu'un brevet émis par une autre autorité.

État antérieur de la technique : technologie utilisée ou publiée antérieurement pouvant être mentionnée dans une demande de brevet ou un rapport d'examen. Il s'agit : i) au sens large, de la technologie qui se rattache à une invention et qui était accessible à tous (car décrite dans une publication ou mise en vente, par exemple) lorsque l'invention a été réalisée; et ii) au sens strict, de toute technologie de ce type susceptible d'invalider un brevet ou de limiter sa portée. Le processus consistant à contester un brevet ou à interpréter ses revendications revient pour une large part à identifier l'état antérieur de la technique et à établir des distinctions entre l'invention revendiquée et cet état antérieur. L'objectif du processus de recherche est d'identifier les documents se rattachant ou non aux brevets qui constituent l'état antérieur de la technique pertinent afin de 
déterminer si l'invention représente une nouveauté et implique une activité inventive.

Famille : ensemble de brevets (ou de demandes) déposés dans plusieurs pays pour protéger la même invention. Ils sont liés entre eux par un ou plusieurs numéros de priorité communs. Il existe différentes définition des familles de brevets (familles de brevets triadiques, familles élargies comprenant des continuations, etc.). Selon l'usage recherché, on choisira un concept de famille ou un autre : équivalents, famille triadique ou famille trilatérale, par exemple.

Familles de brevets triadiques : les familles de brevets triadiques sont définies au sein de l'OCDE comme un groupe de brevets déposés auprès de l'Office européen des brevets (OEB) et le Japan Patent Office (JPO) et délivrés par l'United States Patent \& Trademark Office (USPTO) qui ont en commun une ou plusieurs priorités. Les familles triadiques sont consolidées pour éviter que les brevets déposés auprès de différents bureaux soient comptabilisés deux fois (c'est-àdire, regroupement de toutes les priorités apparentées figurant dans les documents de brevet de l'OEB, du JPO et de l'USPTO).

Familles de brevets trilatérales : une famille de brevets trilatérale fait partie d'un sous-groupe lissé de familles de brevets pour lesquelles une activité de protection par brevet a été démontrée dans tous les blocs trilatéraux. Elle s'apparente donc à une famille triadique, à ceci près qu'elle recouvre aussi les demandes déposées dans un des États contractants de la CBE qui ne sont pas transmises à l'OEB (en plus du JPO et de l'USPTO). Les familles de brevets trilatérales sont généralement comptabilisées en termes de priorités individuelles, sans regroupement.

Japan Patent Office (JPO) : le JPO prend en charge l'examen des demandes et la délivrance des brevets au Japon. Il est rattaché au ministère de l'Économie, du Commerce et de l'Industrie (METI).

Licence : moyen par lequel le propriétaire d'un brevet donne à une autre partie la permission d'accomplir un acte qui, sans cette permission, constituerait une violation du brevet. Une licence peut donc permettre à une autre partie de fabriquer, utiliser ou vendre en toute légitimité une invention protégée par un brevet. En échange, le propriétaire du brevet perçoit habituellement une redevance. L'octroi d'une licence, exclusive ou non, ne vaut pas transfert de la propriété de l'invention au licencié.

Modèle d'utilité : ce type de brevet, également qualifié de " petit brevet » est disponible dans certains pays. Il implique généralement des exigences moins contraignantes pour la brevetabilité qu'un brevet traditionnel, son obtention coûte moins cher et sa validité est plus restreinte.

Non-évidence (USPTO) : on parle d'évidence lorsque les différences existant entre l'objet de la demande de brevet et l'état de la technique sont telles que l'objet dans son ensemble aurait été évident lorsque l'invention a été réalisée 
pour une personne possédant des compétences ordinaires dans la technique dont relève l'objet. Voir également « activité inventive » (OEB, JPO).

Nouveauté : une invention ne peut pas être protégée par un brevet lorsque certains aspects de l'invention ont été rendus publics.

Office européen des brevets (OEB) : l'Office européen des brevets (un bureau régional des brevets) a été créé par la CBE afin de délivrer des brevets européens fondés sur une procédure d'examen centralisée. En déposant une demande unique de brevet européen dans l'une des trois langues officielles (allemand, anglais et français), il est possible d'obtenir des droits de brevets dans l'ensemble des pays contractants de la CBE et de ceux qui autorisent l'extension. L'OEB n'est pas une institution de l'Union européenne.

Opposition : procédure habituellement engagée par des tiers devant le bureau émetteur pour obtenir l'invalidation d'un brevet.

- OEB : une opposition peut être formée contre la délivrance d'un brevet européen dans les neuf mois suivant la publication de la mention de délivrance dans le Bulletin européen des brevets.

- JPO : avant la réforme des recours en invalidation introduite en janvier 2004, une opposition pouvait être formée contre un brevet dansles six mois suivant sa délivrance.

Organisation mondiale de la propriété intellectuelle (OMPI) : organisation intergouvernementale responsable de l'administration de divers traités multilatéraux ayant trait aux aspects juridiques et administratifs de la propriété intellectuelle. Dans le domaine des brevets, l'OMPI est notamment chargée de la gestion de la Convention de Paris, du Traité de coopération en matière de brevets (PCT) et de la Classification internationale des brevets (CIB).

Pays de l'inventeur : pays de résidence de l'inventeur.

Pays de priorité : pays dans lequel la demande de brevet est déposée en premier lieu avant d'être étendue à d'autres. Voir " Convention de Paris ».

Pays désignés : dans les systèmes de brevet internationaux et régionaux, pays dans lesquels les demandeurs souhaitent protéger leur invention en cas de délivrance du brevet. Dans les demandes internationales, sont automatiquement désignés l'ensemble des États contractants du PCT liés par le Traité à la date du dépôt de la demande internationale (depuis 2004). Une règle similaire s'appliquera à l'OEB à compter d'avril 2009, les demandes de brevet européen désignant tous les États contractants de même que dans la procédure PCT.

Premier déposant : système de brevet dans lequel le premier inventeur déposant une demande de brevet pour une invention donnée est titulaire du brevet. Cette loi tend à devenir la norme dans les pays parties à l'ADPIC (Accord sur les aspects des droits de propriété intellectuelle qui touchent au commerce). 
L'OEB et le JPO délivrent des brevets selon le principe du premier déposant, tandis que l'USPTO les attribue selon celui du premier inventeur.

Premier inventeur (USPTO) : système dans lequel un brevet est délivré à la première personne ayant réalisé l'invention même si une autre a déposé une demande de brevet avant elle.

Publication : dans la plupart des pays, une demande de brevet est publiée 18 mois après la date de priorité.

- OEB : toutes les demandes de brevet sont publiées de cette manière, que les brevets aient été délivrés ou non.

- JPO : les demandes de brevet qui ne sont plus en instance auprès du JPO, pour cause de délivrance, de retrait, de renonciation ou de rejet, ne sont pas publiées. Si les bulletins de brevets officiels sont uniquement publiés en japonais, les abrégés et les données bibliographiques de la majorité des demandes de brevet non examinées sont traduites en anglais, et publiées dans les abrégés de brevet japonais (Patent Abstracts of Japan, ou PAJ).

- JUSPTO : avant la modification des dispositions de la loi sur la protection des inventeurs américains (American Inventors Protection Act) de 1999, les demandes de brevet déposées auprès de l'USPTO restaient confidentielles jusqu'à la délivrance. Les demandes déposées le 29 novembre 2000 ou après cette date doivent être publiées 18 mois après la date de priorité. Il existe néanmoins certaines exceptions à la publication des brevets en instance. Ainsi, un demandeur peut solliciter (lors du dépôt) la non-publication du brevet en certifiant que l'invention divulguée dans la demande n'a pas fait et ne fera pas l'objet d'une demande dans un autre pays. De plus, si le brevet n'est plus en instance ou soumis à l'interdiction de divulgation, la demande ne sera pas publiée.

Rapport de recherche : le rapport de recherche est une liste des citations de tous les documents publiés sur l'état antérieur de la technique qui se rapportent à la demande de brevet. Le processus de recherche, mené par un examinateur de brevets, est destiné à identifier les documents liés ou non aux brevets constituant l'état antérieur de la technique qui doit être pris en compte pour déterminer si l'invention représente une nouveauté et implique une activité inventive.

Recherche Euro-PCT (ou Chapitre I PCT) : recherche effectuée par l'OEB agissant en qualité d'administration chargée de la recherche internationale pour une demande Euro-PCT en phase internationale (article $16 \mathrm{du}$ PCT).

Recherche PCT internationale : recherche effectuée par un bureau désigné (administration chargée de la recherche internationale) pour les demandes PCT. 
Recours : procédure par laquelle le demandeur ou titulaire du brevet peut demander l'annulation d'une décision prise par le bureau des brevets.

- USPTO : un demandeur mécontent de la décision du premier examinateur peut, si ses revendications ont été rejetées pour la deuxième fois, former un recours devant le Board of Patent Appeals and Interferences (BPAI) en demandant le réexamen de la décision de l'examinateur. Cette chambre de recours de l'United States Patent \& Trademark Office (USPTO) examine les décisions défavorables des examinateurs relatives aux demandes de brevet et détermine la priorité et la brevetabilité des inventions en cas de recoupement. Un recours peut être formé contre les décisions de la chambre auprès de la Cour d'appel fédérale des États-Unis (Court of Appeals for the Federal Circuit, ou CAFC) ou auprès d'un tribunal d'instance.

- OEB : il est possible de faire appel des décisions des premières instances de l'OEB auprès de ses Chambres de recours, dans le cadre d'une procédure judiciaire (propre à un tribunal administratif) par opposition à une procédure administrative. Ces chambres font office d'instances finales dans les procédures de délivrance et d'opposition devant l'OEB. Outre ces Chambres, l'Office européen des brevets est doté d'une Grande chambre de recours, qui prend des décisions uniquement lorsque la jurisprudence des Chambres de recours présente des contradictions ou qu'une question de droit importante est soulevée.

- JPO : un demandeur confronté à un rejet peut former un recours. Les commissions sont composées de trois ou cinq examinateurs appartenant à la Division d'appel du JPO. Il est possible de faire appel des décisions des commissions auprès de la Haute cour de la propriété intellectuelle, une division spéciale de la Haute cour de Tokyo.

Requête en examen : les demandes de brevet déposées auprès de l'OEB et du JPO ne font pas automatiquement l'objet d'un examen. Le demandeur doit présenter une requête en examen dans les six mois suivant la notification du rapport de recherche pour l'OEB, et dans les trois ans suivant le dépôt auprès du JPO. Les demandes déposées auprès de l'USPTO sont automatiquement instruites par un examinateur sans que le demandeur ait besoin de présenter une requête distincte.

Retrait : conformément à la Convention sur le brevet européen, les demandeurs peuvent retirer leur demande à tous les stades de la procédure. À cet effet, ils peuvent en informer le bureau ou ne pas remplir une ou plusieurs des obligations suivantes : régler les taxes en temps voulu, déposer une requête en examen dans le délai imparti ou répondre en temps utile à toute notification dans le cadre de la procédure d'examen.

Revendication(s) : définition de la portée de l'invention et des aspects de l'invention pour lesquels la protection juridique est demandée. 
Révocation : un brevet est révoqué lorsque, après avoir été délivré par le bureau des brevets, il est jugé non valable par une autorité supérieure (chambre de recours du bureau des brevets ou tribunal).

Taxe de maintien en vigueur (ou de renouvellement) : dès lors qu'un brevet est délivré, une taxe de maintien annuelle doit être versée aux bureaux des brevets pour que le brevet reste en vigueur. Dans la plupart des bureaux, cette taxe est due chaque année. Les brevets (d'utilité) délivrés par l'USPTO sont soumis à une taxe de maintien en vigueur qui doit être versée trois ans et demi, sept ans et demi et onze ans et demi après la date de délivrance initiale.

Traité de coopération en matière de brevets (Patent Cooperation Treaty, ou PCT) : en mars 2008, 138 pays étaient parties à ce traité, signé en 1970 et entré en vigueur en 1978, qui permet aux demandeurs d'obtenir, au moyen d'une procédure unique, un brevet dans une partie ou dans la totalité des États contractants. Le PCT offre la possibilité de demander des droits de brevet dans un grand nombre de pays en déposant une demande internationale unique (demande PCT) auprès d'un seul bureau des brevets (le bureau récepteur). Une telle demande ne donne pas lieu à la délivrance d'un " brevet international ». La décision d'accepter ou de rejeter une demande de brevet revient aux bureaux nationaux ou régionaux. La procédure PCT se décompose en deux grandes phases : i) une phase "internationale "; et ii) une phase " nationale/ régionale » PCT. Les demandes PCT sont gérées par l'Organisation mondiale de la propriété intellectuelle (OMPI).

United States Patent and Trademark Office (USPTO) : l'USPTO est chargé de l'examen des demandes et de la délivrance des brevets aux États-Unis. Il relève de la compétence du ministère du Commerce américain.

Violation : Le fait de fabriquer, utiliser, offrir à la vente ou vendre toute invention protégée par un brevet dans le pays où ce brevet est en vigueur ou d'importer cette invention dans ce même pays pendant la durée du brevet.

Voie Euro-PCT : procédure permettant d'obtenir un brevet européen en désignant l'OEB dans une demande PCT (article 11 du PCT). La première phase de la procédure de délivrance (phase internationale) est régie par le PCT, alors que la phase régionale devant l'OEB, agissant en qualité de bureau désigné ou élu, est régie principalement par la CBE.

- Demande Euro-PCT - phase internationale (ou demande Euro-PCT ou PCT international) : demande PCT désignant l'OEB [article 150(3) de la CBE]; alors que dans la voie Euro-PCT, la première phase de la procédure de délivrance (phase internationale) est soumise au PCT, la phase régionale devant l'OEB, agissant en qualité de bureau désigné ou élu, est régie principalement par la CBE.

- Demande Euro-PCT - phase régionale (ou PCT régional) : demande PCT entrant dans la phase européenne (ou régionale) lorsque le demandeur a 
rempli les conditions requises aux termes de l'article 22 ou $39 \mathrm{du}$ PCT, de l'article 158 et de la Règle 107 de la CBE.

Voie (demande) européenne directe : demande de brevet déposée en vertu de l'article 75 de la CBE (également connue sous le nom de "demande Eurodirecte "). La voie européenne directe implique que l'ensemble de la procédure de délivrance du brevet européen est régie exclusivement par la CBE; dans la variante Euro-PCT, la phase initiale de la procédure de délivrance (phase internationale) est régie conformément aux dispositions du PCT. 
LES ÉDITIONS DE L'OCDE, 2, rue André-Pascal, 75775 PARIS CEDEX 16 IMPRIMÉ EN FRANCE

(92 2009022 P) ISBN 978-92-64-05415-8 - nº 565362009 


\section{Manuel de l'OCDE sur les statistiques des brevets}

Les données de brevets sont une source très riche d'information pour l'étude du changement technique. Parallèlement à d'autres indicateurs de science et technologie (S-T) telles les dépenses ou les personnels de R-D ou les enquêtes innovation, les données de brevets fournissent des informations détaillées sur l'activité inventive et les multiples dimensions des processus d'invention (par exemple sur la localisation géographique, l'origine technique ou institutionnelle, les individus et les réseaux). De plus, les données de brevets permettent des comparaisons temporelles et internationales. Cependant, ces données sont complexes, et les indicateurs de brevets doivent être conçus et interprétés avec prudence. Depuis la publication du manuel de l'OCDE sur les brevets en 1994 (La mesure des activités scientifiques et technologiques : les données sur les brevets d'invention et leur utilisation comme indicateurs de la science et de la technologie), des progrès significatifs ont été réalisés, aussi bien en termes de disponibilité des données que d'analyse des données de brevets.

L'édition 2009 du Manuel de l'OCDE sur les statistiques des brevets intègre les développements récents du domaine. Elle fournit des lignes directrices pour l'utilisation des données de brevets à des fins de mesure de S-T, et des recommandations pour la compilation et l'interprétation des indicateurs de brevets dans ce contexte. Le manuel vise à montrer à quoi les données de brevets peuvent ou ne peuvent pas être utilisées, et comment compter les brevets de façon à maximiser l'information sur la S-T tout en minimisant le bruit et les biais statistiques. Enfin, il décrit comment les données de brevets peuvent être utilisées dans l'analyse d'un large spectre de thèmes liés au changement technique et à la prise de brevets, y compris les liens science-industrie, les stratégies de brevets des entreprises, l'internationalisation de la recherche, et la mesure de la valeur des brevets.

\footnotetext{
Le texte complet de cet ouvrage est disponible en ligne aux adresses suivantes : www.sourceocde.org/scienceTI/9789264054158 www.sourceocde.org/statistiquessourcesmethodes/9789264054158
}

Les utilisateurs ayant accès à tous les ouvrages en ligne de l'OCDE peuvent également y accéder via : www.sourceocde.org/9789264054158

SourceOCDE est une bibliothèque en ligne qui a reçu plusieurs récompenses. Elle contient les livres, périodiques et bases de données statistiques de l'OCDE. Pour plus d'informations sur ce service ou pour obtenir un accès temporaire gratuit, veuillez contacter votre bibliothécaire ou SourceOECD@oecd.org. 